


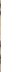

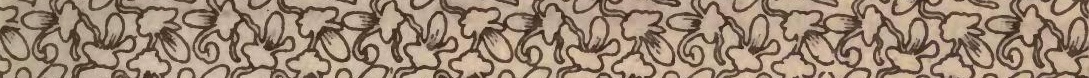
C. (2)

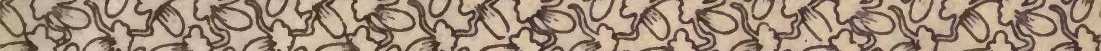

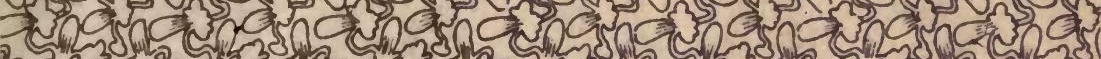

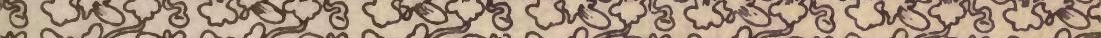
2.

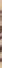

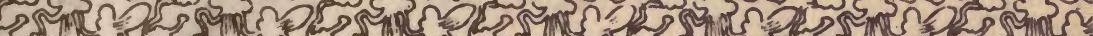

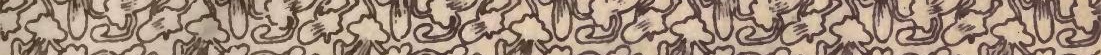

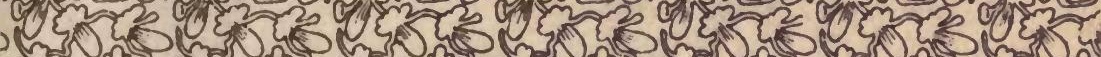
$=30,30$

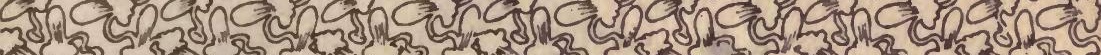

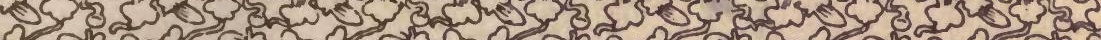

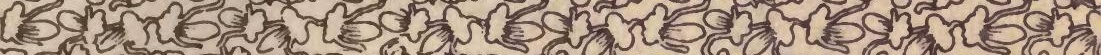

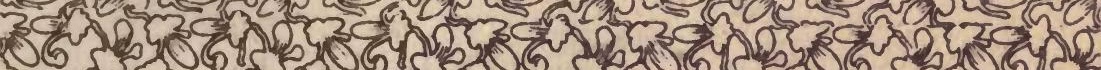

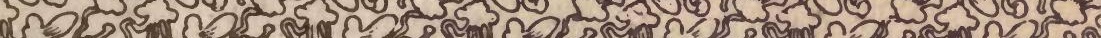
240302030

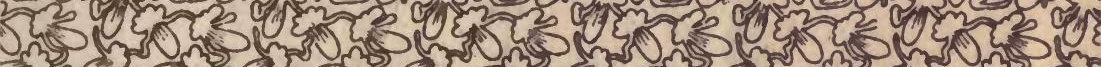
(1)

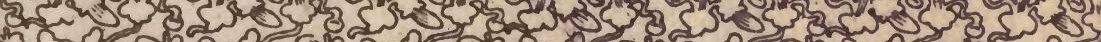

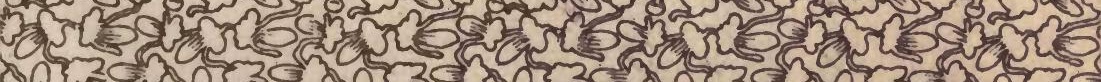

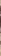

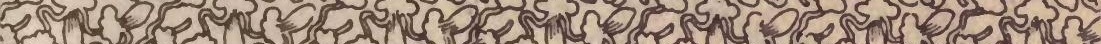

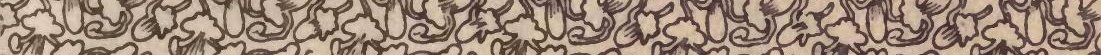

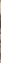
30,300

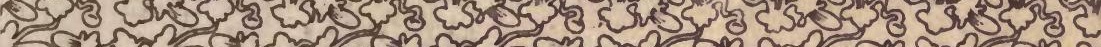
50 ,

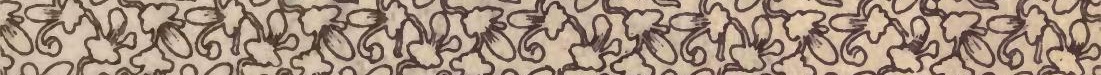
(2) 35 (n)

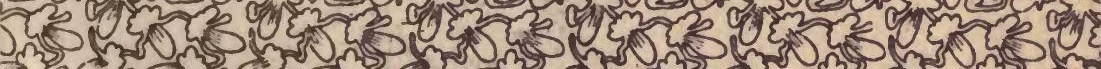

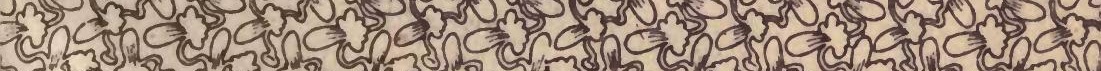

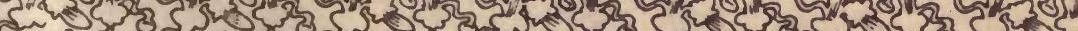
3.

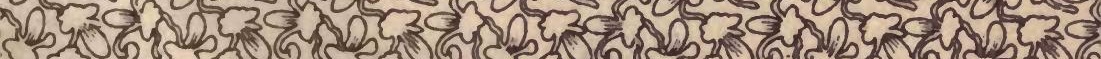

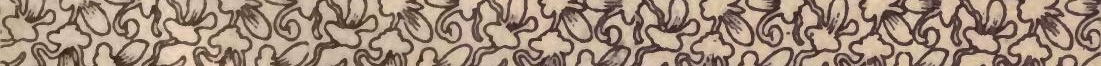

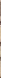

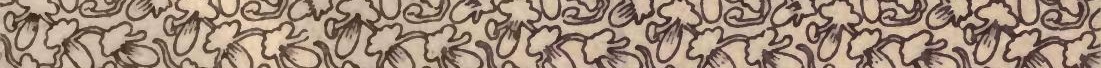

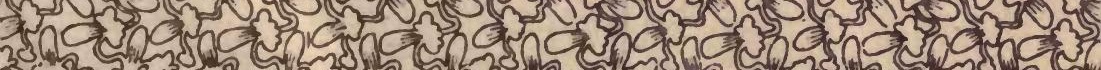

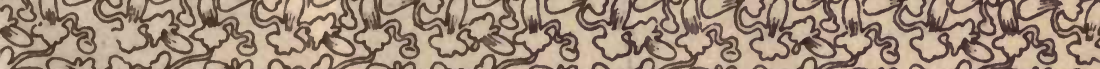

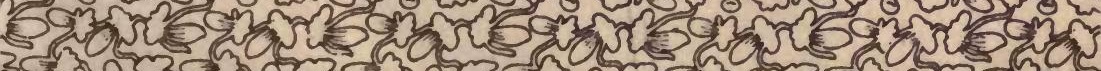

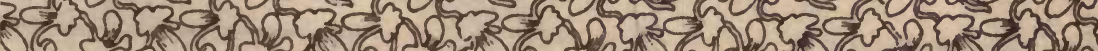

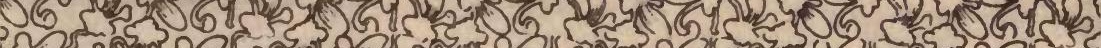

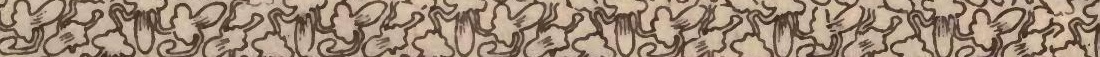




$$
\text { po } \begin{aligned}
& 1-110 \\
& 188-905
\end{aligned}
$$




\title{
AN INTRODUCTION TO THE STUDY OF _CYTOLOGY
}

\author{
BY \\ L. DONCASTER, Sc.D., F.R.S. \\ FELLOW OF KING'S COLLEGE, CAMBRIDGE \\ DERBY PROFESSOR OF ZOOLOGY IN \\ THE UNIVERSITY OF LIVERPOOL
}

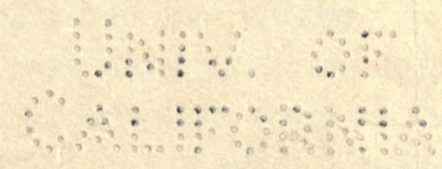

\section{CAMBRIDGE}

AT THE UNIVERSITY PRESS

$$
\text { I } 920
$$




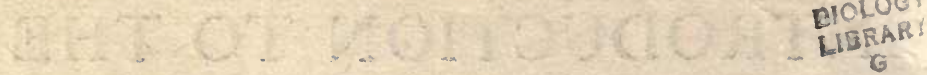

$\because \because \because \quad \because \because \vdots \vdots \vdots \vdots \vdots$

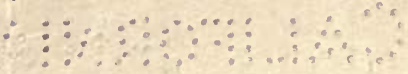




\section{PREFACE}

$A^{\mathrm{S}}$ its title is intended to indicate, the scope of this book $\mathrm{Al}$ is not precisely that of a text-book. Generally speaking, the purpose of a text-book, especially in a biological subject, is to give a concise summary of the known facts, and perhaps of established inferences from the facts, but not to enter at any length into theoretical discussions, especially when the leading investigators of the subject are not yet in agreement upon their interpretation. The aim of this book is rather different. While attempting to give an account of the more important facts of animal cytology, its chief purpose is to interest the student in the subject by pointing out some of the ways in which cytological investigation is related to the great fundamental problems that lie at the root of all biological research. Some minds are naturally interested in facts for their own sake, but to these a rather specialised study like cytology is hardly likely to make a strong appeal, and if, as the writer believes, cytological investigation in the widest sense of the word offers one of the most hopeful lines of advance in biological knowledge, it seems essential that the bearing of cytological facts on more general problems should be considered in any introductory course on the subject.

The plan of the book is based on that of the courses of lectures given by the writer during some six years at Cambridge. Those courses have naturally varied somewhat from year to year; the subject has made very great advances 
during the period, and the experience of each year has led to modifications in the years that followed. But the general plan has remained the same, and the writer believes that it has proved to be of a kind to attract the interest of students not previously familiar with the subject, and that therefore a book of the kind might prove to be of value to students in other laboratories.

It is almost inevitable that a book of this kind should reflect the special interests and prepossessions of the writer. Cytology lies at the base of every biological phenomenon, and at least to one engaged in biological research some aspects of the subject must appear more interesting and important than others. To attempt complete impartiality, and to discuss every kind of theoretical application of cytological facts with equal thoroughness, would be an impossible task even in a much larger book, and would probably lead to a very uninspiring presentation of the subject. While trying, therefore, to point out the importance of various aspects of cytological study, it is possible that the book errs in devoting too much space to those sides of the subject in which the writer is chiefly interested, especially the cytological basis of hereditary transmission and of sexdetermination. But even if it may appear to some that the treatment is lop-sided in this respect, one good excuse may be urged which is not of a personal nature. By far the greater part of cytological research during the past fifteen years has been devoted, directly or indirectly, to these questions, and though in years to come it may seem, quite rightly, that cytologists have been far too exclusively occupied with one group of problems, it is hardly possible at the present time not to emphasise them, even at the expense of others that are in fact of no less importance. If the treatment is lop-sided, it may fairly be said that it reflects the 
lop-sidedness of recent cytological work, but one of the aims of the book is to indicate that many other problems of equal interest remain to be investigated.

In a book of this size it is impossible to mention a large part of the facts, and many investigations of the first importance are not referred to. The aim has been rather to give representative examples, and the student who wishes to pursue the matter further will have little difficulty in finding the necessary references in the papers given in the bibliography. This list includes few papers not referred to in the text, but is intended to be sufficiently representative to enable the student to find the other literature that he may need. Although much has been omitted, the attempt to state the facts that are mentioned in as small compass as possible has necessitated rather serious condensation, and the writer is painfully aware that some parts of the book do not offer such easy reading as they might have done had he allowed himself more space.

In choosing figures for the illustrations, an attempt has been made to employ as far as possible copies of original figures, and to make use of diagrams only when they seem necessary for a clear comprehension of the subject. Works on cytology in the past have seemed to the writer to err in the too frequent employment of diagrams, which sometimes seem to the novice to bear no very close relation to the actual figures that he sees with the microscope, and though the methods of reproduction employed have necessitated a certain amount of simplification in some cases, it is hoped that the use of figures from original papers may to some extent avoid this defect. A few of the purely diagrammatic figures are new; others are borrowed from well-known books and articles. In every case the source of borrowed figures is acknowledged, and the reference given in the literature 


\section{PREFACE}

list. Thanks for permission to reproduce figures are especially due to Prof. T. H. Morgan and Prof. E. B. Wilson of Columbia University, to the Editors of The Quarterly Fournal of Microscopical Science, The Journal of Experimental Zoology, The Fournal of Morphology, and the Bio- logical Bulletin, and, for the loan of original drawings for reproduction, to Dr W. E. Agar and Dr C. B. Bridges.

L. D.

\section{Cambridge,}

August, 1919. 


\section{CONTENTS}

CHAP.

I.

The Cell .

PAGE

Definition-The cell theory-Protoplasm-Fixation and staining.

II. Cell Organs, the Nucleus, Mitochondria and Golgi Apparatus .

The nucleus; its functions-Nucleo-plasmatic relation - Structure of the nucleus-Chromatin-Mitochondria - Golgi bodies.

III.

\section{Cell Division}

Amitotic division-Mitotic division or KaryokinesisChromosomes-A chromatic spindle-Chromosomes in mitosis-Cytoplasmic division.

IV. The Centrosomes, Spindle and Asters . .

The centrosome and its origin-The achromatic spindle-Mechanism of mitosis-The spindle and cytoplasmic division-Multipolar spindles.

v.

The Maturation of the Germ-cellis; SperMATOGENESIS . . . . . •

Chromosome reduction-Comparison of spermatogenesis and oogenesis-Meiotic phase-Spermatogenesis of Lepidosiven-Terminology-Origin of bivalent chromosomes-Tetrads-Segregation of chromosomes.

Vi. The Maturation of the Germ-cells (continued). The Maturation of the Egg and the Nature of the Meiotic Phase . .

The ovarian egg-Deposition of yolk-Polar divisions - Fate of polar nuclei-The meiotic pairing of chromosomes-Interpretation of meiosis-Chiasmatypy. 
VII. The Cytoplasmic Structures in SpermatoGenesis AND Oogenesis

The spermatozoon-Its development-MitochondriaThe acrosome and Golgi apparatus-Oligopyrene and apyrene spermatozoa-Accessory cells-The eggYolk and nutritive cells-Egg membranes.

VIII. Fertilisation and Segmentation

Entrance of spermatozoon-Fertilisation membrane and prevention of polyspermy-Conjugation of nuclei - Segmentation-Position of cleavage planes-Centrolecithal segmentation-Polyspermy.

IX. Parthenogenesis . . . . . .

Parthenogenetic eggs with one polar division-Fertilisation by second polar nucleus-Partrenogenetic eggs with two polar divisions-HymenopteraPhasmidae-Gall-flies-False fertilisation-Summary.

X. Artificial Parthenogenesis . . . . Methods-Theoretical explanations-Comparison with fertilisation-Chromosomes and centrosomesArtificial parthenogenesis in frogs-Sex.

XI. The Cytological Basis of Sex-Determination Hymenoptera: haploid and diploid chromosome series - Heterochromosomes-Idiochromosomes-Life-cycle of Aphids and Phylloxerans-Hermaphroditism: Rhabdonema-Lepidoptera.

XII. Germ-cell Determinants . . .

Germ-plasm and germ-track-Germ-cells of AscarisOf Sagitta-Miastor-Pole-plasm in other InsectsOosoma of Litomastix-Ectosomes of CopepodaOocyte of Dytiscus-Sertoli cells-Nature of germ-cell determinants.

XIII. The Theory of the Individuality of the Chromosomes

The hypothesis of individuality-Evidence from abnormal numbers-Persistence of chromosomes in the resting nucleus-Differences of size and shapeHybrids-Differences of function of chromosomesMicrosomes as units. 
Weismann's theory-The nucleus in heredity-Experiments of Boveri and of Herbst-Chromosome elimination in hybrids-Oenothera-Chromosome behaviour and Mendelian segregation-Objections to the hypothesis-Gametic coupling (linkage)-Drosophila - Crossing-over and chiasmatypy-Sex-limited (sexlinked) inheritance-Non-disjunction-Linkage in male and female-Chemical analogy-Summary.

XV. The Rôle of the Cytoplasm in Development AND HeREDity . . . . . .

Hybridisation experiments-Sperm nucleus in foreign egg cytoplasm-Degrees of incompatibility-Cytoplasmic transmission-Differential cell divisionOrgan-forming substances-Centrifuge experimentsEquipotential systems-Summary.

\section{Conclusion}

Structure and function-The problem of differentiation-Cytology and Physical Sciences-Comparative Cytology-Origin of life-Methods of investigation.

BIBLIOGRAPHY 


\section{LIST OF PLATES AND TEXT-FIGURES}

\section{PLATES}

PLATE

I. Echinus larvae from nucleated and non-nucleated egg-fragments, after Boveri. Amitotic nuclear division, from MARSHALL . .

II. Stages of mitotic division in Sea-urchin egg, from Wilson and LEAming . . . .

III. Origin of centrosomes and spindle in the egg of the Axolotl, after Jenkinson . . .

IV. Stages of the maturation divisions (meiosis) in the spermatogenesis of Lepidosiven, from AGAR .

V. Prophases of the heterotype division in spermatocytes of the Guinea-pig, after MOORE and WALKER.

Heterotype mitoses in spermatocytes of Stenobothrus, after DAvis . . . . .

VI. Diagrams illustrating the hypotheses of telosyndesis and parasyndesis, after GRÉGOIRE .

VII. Maturation divisions with Tetrad formation in Cyclops, from RücKERT

VIII. Development of the oocyte in Paludina, after PopofF

IX. Production of polar bodies in the egg of Physa, from KostaNECKI and WIERZEJSKI

X. Conversion of the spermatid into the spermatozoon in Euschistus, after MONTGOMERY.

Sertoli cells in testicular tubule of the Rat, from KORSCHELT and HEIDER.

Apyrene spermatozoon of Vermetus, after KuschaKEWITSCH

XI. Mitochondria in spermatogenesis of Lepidoptera, rom GATENBY

XII. Stages of fertilisation of the egg of the Sea-urchin, from Wilson and LeAMING . . . .

XIII. Oogenesis and spermatogenesis in Aphids, after MORGAN

XIV. Spermatogenesis of the Hornet, after MEves - p. I34

XV. Spermatogenesis of the Hive Bee, from MARK and COPELAND 


\section{PLATES AND TEXT-FIGURES}

xiii

PLATE

To face

XVI. Life-history of the Gall-fly Neuroterus . p. 136

XVII. Hetero- and Idio-chromosomes in Hemiptera, from WrLson

XVIII. Chromosome-groups of Metapodius, from Wrison

p. 156

p. 160

XIX. Chromosome-cycle in Phyllaphis . . . . p. I63

$\mathrm{XX}$. Chromosomes in the development of eggs and spermatozoa in Rhabdonema nigrovenosum, after BOVERI

XXI. Chromatin diminution in segmentation of the egg of Ascaris, after BoverI . . . . p. 174

XXII. Germ-cell determinant in segmenting egg of Sagitta, after ELPATIEWSKY.

Ectosomes in segmenting egg of Cyclops, after Aмma

p. 176

p. 178

XXIII. Segmentation of the egg of Miastor, after HeGneR

XXIV. Development of Dentalium, showing effects of removal of yolk-lobe, from WiLson • • . p. 249

\section{TEXT-FIGURES}

FIG.

PAGE

r. Mitochondria in the spermatogenesis of Blaps, after DUESBERG

2. Golgi apparatus in epidermal cells, after DeINEcKA

3. Equatorial plates showing arrangement of chromosomes, from PAYNE and ROBERTSON . . . .

4 Telophase in pollen division of Paris, after Bolles LEE .

5. Diagram of eggs segmenting normally and compressed in glass tubes

6. Tripolar and quadripolar spindles in Echinoid eggs, after GRAY

7. Diagrams of spermatogenesis and oogenesis, after GRÉGOIRE

8. Types of Insect ovarian tubes, after Korschelt and HEIDER . . . . . . . .

9. Stages in the maturation of the egg of the Saw-fly Nematus

10. Early development of the egg of Platygaster, showing origin of the trophamnion from the polar nuclei, after Silvestri and MARCHAL

II. Chromosomes of Biston hirtarius and Nyssia zonaria, from HARRISON and DONCASTER

12. Diagram of chromosome division in somatic and maturation mitoses 
FIG.

13. Diagram illustrating JANSSENs' hypothesis of chiasmatypy

14. Diagram of a spermatozoon .

I5. Verson's cell in testis of Pieris

I6. Fertilisation by second polar nucleus in artificially parthenogenetic egg of a Star-fish, after BuCHNER

17. Chromosome-groups in the Gall-fly Neuroterus

I8. Compound groups formed by union of the $X$-chromosome with other chromosomes in Orthoptera, from WiLson

I9. Chromosomes in the maturation of the eggs of Moths, after SEILER and DONCASTER

20. Early development of the egg of Litomastix, after SIL VESTRI

21. Development of oocytes and nurse-cells in Dytiscus, after GÜNTHERT

22. Differentiation of a Sertoli cell from spermatogonia in Man, after Montgomery .

23. Persistence of abnormal chromosome number in segmentation of egg of Ascaris, from BoverI .

24. Nuclei in segmentation of the egg of Ascaris, from BovERI

25. Chromosomes emerging from nuclear network in spermatogonia and spermatocytes of Hemiptera, from WiLsoN

26. Diagram of distribution of chromosomes on a quadripolar spindle, from BoverI

27. "Partially thelykaryotic" pluteus of Echinoid hybrid, from HERBST .

28. Diagrams illustrating the hypothesis of "crossing-over," from MORGAN.

29. Chromosome groups of Drosophila showing non-disjunction, from original drawings by BRIDGES

3o. Eggs of Neveis segmenting normally and under pressure, from WiLson

3r. Segmentation of centrifuged eggs of Arbacia, from 


\section{AN INTRODUCTION TO THE STUDY OF CYTOLOGY}

\section{CHAPTER I}

\section{The Cell}

YYTOLOGY may be defined as the study of cells; it 1 differs from the related science of Histology in dealing with cells as units, while histology is concerned with cells in larger aggregates, and is rather the study of tissues. A serious defect, however, in this definition of cytology is that it involves the term "cell," a word which at once opens up a wide field of controversy and misunderstanding. As long as it was generally agreed that all organisms are built up of cells much as a house is built of bricks, the description of the cell as "a unit of living matter" was not open to any very grave objection; the cell was to the biologist almost what the atom was to the chemist-the smallest portion of living matter capable of an independent existence-and the word had, in appearance at least, a fairly definite meaning. Now, however, when the old idea of discrete and independent cells is almost abandoned, and when distinguished biologists maintain that one whole group of organisms (the Protista) are non-cellular, the word "cell" is beginning to lose its' definite and precise significance, and to be used rather as a convenient descriptive term than as denoting a fundamental concept of biology.

The cell-theory arose, of course, from observation of the structure of plants. A section of an ordinary plant shows

D. C. 
the tissues composed of chambers with definite walls, and these chambers were not inappropriately named cells, and the substance of the walls cellulose. Very little investigation was needed, however, to show that the essential feature as far as life is concerned was not the walls, which are mainly skeletal in function, but the contents, and that each cell contained a certain quantity of protoplasm and a nucleus. Frequently, of course, there are other structureschloroplasts, vacuole, and so forth-but since the almost invariable and therefore presumably essential feature of the cell is the presence of protoplasm containing a single nucleus, and since these same features are found in animals in the absence of cellulose walls, or indeed of definite walls of any kind, the meaning of the word "cell," by a natural transition, became changed to "a mass of protoplasm containing a nucleus." Such a definition is certainly better than "a unit of living matter" because it involves no theoretical implication, but it is still far from satisfactory for various reasons. In the first place, frequently in the Protozoa, and not very rarely in other animals, cells, or at least definite masses of protoplasm, exist, which contain two or more nuclei. Secondly, bodies that can hardly be denied the name of cells may either apparently lack a nucleus and nuclear matter altogether, as for example the red blood-corpuscles of Mammals, or may have nuclear matter distributed through them without forming a definite nucleus, as in some Protozoa. And lastly, histological investigation shows more and more clearly that even when cells appear to be sharply defined and distinct, there are nevertheless connections between them, and that to regard the organism as built up of discrete cells which co-operate physiologically but are fundamentally independent is a false conception-almost as false as to regard the surface of the sea as made up of a series of 
independent waves, instead of regarding the waves as a partial interference with an underlying continuity.

The cell theory in its original and crude form regarded an organism as composed of a horde of discrete units which co-operate for a common purpose and are modified in various ways to make that co-operation more effective, much as a human community consists of many separate individuals, having different occupations and co-operating for their common good. According to this idea the individuality of any organism arose from an integration of the individualities of its separate cells, and is thus a corporate individuality such as may exist in a school or a regiment. Nowadays, however, opinion tends in the opposite direction - to regard the organism as the individual, with a common life running through it all, and the cells not as units of which it is built up but rather as parts into which it is divided in order to provide for the necessary division of labour involved in so complex a process as life. The conception of the cell thus remains, but no longer requires or is capable of the strict definition that was needed when the word was supposed to represent a fundamental biological entity. Organisms may be non-cellular if they are not divided up into cells; portions of organisms may exist which are not strictly cells in the old sense of the word (for example Mammalian red blood-corpuscles) and yet have so much the character of cells that the term may well be applied to them. The word, in fact, may conveniently remain as a useful descriptive term, implying as a rule a portion of protoplasm containing a nucleus in immediate physiological connection with it, but from which either the nucleus or perhaps even the surrounding protoplasm may sometimes be absent.

Although the theory of the independence and individu- 
ality of cells is now being generally, if gradually, abandoned, it sometimes tends to reappear in the form that the nucleus is the fundamental and all-important entity, and that although the cell-protoplasm ("cytoplasm") of the body is more or less continuous, the nuclei constitute distinct and independent units, in some way controlling and acting through the protoplasm which surrounds them, but all, to a large extent at least, unconnected with one another. This view has the advantage that it is not at variance with such obvious facts as the existence of multinucleate cells, and is supported by the usually sharply marked and definite character of the nucleus, but it does not take into account the reciprocal relation that exists between nucleus and cytoplasm. It also seems inconsistent with the fact that at times the nuclear substance may be more or less distributed through the cell, and that in the Protozoa at least a typical nucleus may be reconstituted from finely-divided fragments of nuclear substance to which by themselves it seems impossible to apply the term nucleus. A great part of this volume will be devoted to the subject of the nucleus, so it will not be necessary here to say more than that this view does not seem adequately to meet the objections to the cell-theory in its older forms, and that it is probably better to regard not only cells but also nuclei as rather parts of an individual whole than to think of them as units out of which that whole has been built up.

Even if the cell is no longer regarded as a fundamental unit in the composition of all living organisms, it is nevertheless a convenient and quite natural conception, for the bodies of organisms consist of protoplasm, or its various products and modifications, in which are embedded nuclei, and each nucleus controls, or is in relation with, a certain amount of the surrounding protoplasm. The protoplasm 
may thus be divided up into masses, sometimes distinct and sometimes continuous, each of which is in relation with one nucleus, and such a nucleated portion of protoplasm constitutes a cell. The word protoplasm is commonly used as a general designation for the various substances of which both nucleus and cell-body consist, and which together constitute "living matter," while the cell-body or extra-nuclear protoplasm is distinguished as cytoplasm. Probably the nuclear substance and certainly the cytoplasm undergo very various modifications in different kinds of tissues, and the cytoplasm frequently contains inclusions of various sorts produced by its own activity, such as the yolk granules in an egg, the fat globules in adipose cells, the secretion in gland cells, etc. These various inclusions produced by the cytoplasm, but not actually part of it, are often designated collectively as "deutoplasmic"; the word "metaplastic" has also been used. Some cells also contain larger or smaller cavities filled with watery fluid, known as vacuoles, which play an important part in plant cells and in certain skeletal tissues of animals. In a relatively unmodified embryonic cell, however, the cytoplasm is a clear, semifluid, colourless substance resembling egg-albumen, and it is to this substance that the word protoplasm in its stricter sense is applied.

Although the protoplasm of an unmodified cell may appear homogeneous, it undoubtedly consists of a complex mixture, and the question of its structure has given rise to much controversy. In the practical study of cytology it is generally necessary to examine structures after they have been "fixed" and stained, that is to say, after the tissues have been killed and coagulated by reagents, and stained with dyes in order to differentiate the various parts which in the living cell are barely distinguishable from one 
another. But it must always be remembered that the process of fixation, and possibly also the subsequent embedding, section-cutting, mounting, and staining, may produce important artificial modifications in the original structure, or even give rise to structure which did not exist in the living cell. A comparison of the appearance produced by different fixatives, and of the fixed with the living cell, shows that in general the coarser and more obvious structures are probably in most cases faithfully preserved by suitable fixatives, but when one comes to the finer details it is extremely difficult to be sure that they in any way represent the true structure as it existed in life. In the living cell all the parts are commonly colourless and transparent, and are therefore only distinguishable by differences in refrangibility, and such differences are visible only in the case of bodies of a certain size. In the sections of the fixed and stained cell many much finer details are easily seen, but in interpreting these attention must always be paid to the possibility that they have undergone more or less serious modification in the process of preservation, or even that they are purely artificial. That this danger is not an imaginary one is easily realised by comparing preparations of the same tissue fixed and stained by different methods, or even by noting the variations in different parts of a single preparation, in cases in which the fixative is not very penetrating, and in which the outer portions may have a quite different appearance from those at a greater depth from the surface. Even when the various methods used all result in what are called "well-fixed" preparations, quite considerable differences may follow the different proceedings, and in "badly-fixed" tissues the existence of purely artificial structures is often perfectly evident. In practice, however, the difficulty of determining whether a structure is natural 
or artificial is usually not very serious in the majority of bodies with which we shall have to deal; a comparison of the results of various methods of fixation, and, when possible, of these with the living cell itself, is usually sufficient to give at least a clear indication of the truth or falsity of the representation seen in the stained preparation. But it is important to remember that the preparations studied by the cytologist are only representations or pictures of reality, and not the reality itself; the real cell consists of viscid, or at best semi-solid, substances suspended in a still more fluid medium, while in the stained section these substances are coagulated and rendered distinct by differential staining, and it is hardly possible that this process can take place without considerable deformation. Some portions of the cell will be contracted and shrivelled, some swollen, and some perhaps removed altogether, and careful comparison of the results of different methods is needed before a true idea can be obtained of the original form.

Although the examination of the living cell gives good ground for the belief that on the whole the methods used by cytologists do in fact give authentic pictures of its real structure, especially with regard to the nucleus and the phenomena of nuclear division and also to the bodies embedded in the cytoplasm, yet when these methods are applied to the structure of protoplasm itself the doubt on this point becomes a very real one. The reason for this is that different methods of fixation give different appearances, and the structure is so fine that it is practically impossible to check the results by comparison with the protoplasm of the living cell. There are four main hypotheses as to the structure of protoplasm, three of which assume that it consists of a more solid substance embedded in a more fluid medium, while the fourth regards protoplasm as 
practically structureless. The first of the three hypotheses is that protoplasm consists of a relatively solid network or reticulum enclosed in a fluid, like a sponge in water; this may be called the reticular hypothesis of Flemming. The second, or alveolar hypothesis, of Bütschli, regards the more solid portion as forming a sort of foam, enclosing the fluid in the cavities or alveoli. In thin sections, of course, the alveolar and reticular structures would give similar appearances, but they differ in the fact that in a reticulum the fluid is continuous, while in a foam structure the cavities are closed so that the fluid is divided into a number of separate compartments. The third hypothesis is that of Altman n, which regards the more solid portion as having the form of granules, suspended as in an emulsion in a fluid medium. Each of these three hypotheses is in agreement with the facts, in the sense that either reticular, alveolar or granular structure may be obtained in fixed preparations of protoplasm, according to the material chosen and the fixative employed. But since each type of structure may be obtained in apparently well-preserved material, there is no satisfactory means of choosing between them, since the structure is too fine for definite results to be obtained from an examination of living protoplasm. Under these circumstances the doubt naturally arises whether any of the three represents the real condition as it exists in life, and whether the apparent structures seen in fixed material may not be due to the process of coagulation under the influence of different fixatives. The probability that this is so is greatly increased by the results obtained by HARDY (1899) in the study of the action of different fixatives on a variety of colloidal solutions. HARDY found that when a solution of a colloidal substance is caused to coagulate, the more solid portion separates from the fluid with 
which it had been intimately mixed, and that the form taken by the solid depends on the strength of the original solution and on the nature both of the colloid substance and of the fixative. If the solution is weak, when it coagulates the solid takes the form of granules; when it is stronger, it may form either a network or a foam-structure according. to whether the coagulum is soluble or insoluble. For example, a gelatin solution coagulated with sublimate forms a soluble coagulum which has an alveolar structure, while albumen treated with sublimate, or gelatin with formalin, forms an insoluble coagulum which is reticular in structure. HARDY further found that if fine particles are suspended in the solution, they come to lie at the nodes of the mesh- or foam-work when the solution is coagulated, and that the size of the particles determines the fineness or coarseness of the meshes. Applying these observations to cell-structures, he suggests that the mesh-work commonly found in fixed nuclei is due simply to the effects of fixation, and that the size of the meshes depends on the fineness of division of the chromatin particles in the nucleus. Under some circumstances he was able to fix nuclei with no meshwork at all, but merely with granules of chromatin and other substances. As will be seen later, it seems possible at least in some conditions of the nucleus to see a mesh-work in the living cell, but in general HARDY's experiments seem to indicate that living protoplasm has none of the structures that have been ascribed to it, and that, apart from the bodies which are almost always included in it, it is structureless. As a matter of fact, the three chief hypotheses of the structure of protoplasm (reticular, alveolar and granular) all depend very largely, for their observational basis, on investigations upon protoplasm containing quite definite inclusions. Many observers, for example, have regarded the Echino- 
derm egg as affording an instance in which clearly defined structure could be studied, but E. B. Wilson (1900, p. 25) has shown conclusively that these eggs consist of an emulsion of larger and smaller fluid droplets in an apparently homogeneous ground-substance. It is impossible to draw any real distinction between the larger of these droplets and the yolk granules of other animals, and although it is certain that these eggs have an alveolar structure (the droplets forming the alveoli) it is hardly possible to regard this structure as representing that of protoplasm, for the protoplasm, properly speaking, is confined to the apparently homogeneous substance in which the droplets are embedded. Con KLIN finds that in the egg of Crepidula this protoplasmic ground-substance is by no means fluid, but is elastic and contractile, so that when the egg is distorted by being centrifuged it "tends to come back to its normal form and to bring back to their normal positions displaced constituents of the cell1."

The statement that protoplasm is structureless does not imply that it is a homogeneous chemical compound, for it is undoubtedly a highly complex and unstable mixture; it means that it consists of a number of colloidal substances in solution, and so intimately mixed that no mechanical structure exists which can be detected by the microscope. Moreover, these observations apply only to unmodified protoplasm as seen in an undifferentiated embryonic cell, and even in such a cell there are practically always inclusions of various sorts consisting partly of substances produced or secreted by the cell and used by it in its further development ("deutoplasm"), and partly of substances which seem to have a more vital connection with the cell's activity, and which may almost be regarded as cell-organs

${ }^{1}$ E. G. Conkuin (1917). 
(mitochondria, Golgi apparatus, etc.). In cells which have begun to be differentiated to form tissues of any sort, some kind of cell-structure is almost always present, but this must be regarded as the structure of the cêll and characteristic of the particular tissue rather than as protoplasmic structure as such. Some examples of such cell-structures will be referred to later, but in general they are the concern of the histologist rather than of the cytologist, and will not be dealt with fully. It will also be convenient to postpone any further consideration of cytoplasmic structures until some account has been given of the nucleus. First, however, it will be advisable to devote a short space to the different kinds of stains commonly used to differentiate the various structures in the cell.

A very large variety of stains has been used in cytological work, but in general these may be classified in to two groups, known as acid and basic. "Acid" stains do not necessarily give an acid reaction with indicators such as litmus, but tend to combine with substances which have the chemical constitution of bases, while basic stains combine with organic acids. In general basic dyes are used chiefly for staining the chromatin of the nucleus, but stain also certain kinds of inclusions in the cytoplasm, while acid stains are taken up by the non-chromatinic parts of the nucleus and by most cytoplasmic structures. It should be noted, however, that the staining reactions depend to a considerable extent on the fixatives used, and especially on whether the tissues have previously been treated with acid or alkaline solution. Most fixatives are acid, and the staining effects mentioned are those which normally follow the use of such fixatives. When only one stain is used, it is commonly taken up to some extent by almost all parts of the cell, though it usually stains some parts more intensely than others; but when 
a suitable mixture of differently coloured acid and basic stains is used, or if the section is treated with one in succession to the other, it is often possible to get very sharp differentiation of the various constituents of the cell. Structures which take acid stains are spoken of as oxyphil, those which take basic stains as basiphil. In addition to the stains which are more or less specific for chromatin, but - which are also taken up by certain other structures, various special stains are used for particular purposes, e.g. to indicate the presence of fat or other inclusions or secretions of the cytoplasm. Since, however, comparatively few stains are absolutely specific, it is advisable in most cases to test any doubtful body with more than one; otherwise it may happen that, for example, a body is identified as consisting of chromatin, when it is really an inclusion of some quite different nature. 


\section{CHAPTER II}

\section{Cell Organs, The Nucleus, Mitochondria and Golgi Apparatus}

$T^{N}$ discussing the cell-theory it was concluded that the only satisfactory general description of a cell is "a nucleated portion of protoplasm," and this description or definition indicates the important part played by the nucleus in the life of the cell. It is not the purpose of this book to discuss at all fully the problems of cell-physiology, but it may not be out of place to say a few words on the function of the nucleus. The nucleus seems to act as the centre which controls the metabolic activities of the cell; a non-nucleated portion of protoplasm can neither absorb nourishment nor secrete. If a ciliated Protozoon is cut in two, in such a way that one half contains the nucleus, the nucleated portion can regenerate or repair the missing part, but the half which lacks any nuclear matter is unable to repair the wound. The cilia continue to move for a time, so that the power of locomotion remains, and it may engulf food, but since the food is not digested and no regeneration takes place, it soon dies. Again, it has been shown that the nucleus is necessary for the secretion of a cell-wall in the plant cell by an ingenious experiment of TOWNSEND. He placed root-hairs of Marchantia in hypertonic solution of salt so that plasmolysis took place, i.e. owing to the withdrawal of fluid from the vacuole by osmosis, the protoplasm was drawn away from the cell-wall and became aggregated into naked masses. Some of these masses were nucleated, some were without nuclei but were connected by fine strands 
of protoplasm to a neighbouring nucleated mass, and some were without nuclei and quite separate. The nucleated masses, and also those with no nucleus which were connected by a strand to a nucleated mass, both after a time secreted new cell-walls around themselves, but the non-nucleate masses produced no cell-wall and gradually died. Another interesting observation showing the influence of the nucleus on the cell-protoplasm was made by Schaudinn. He found that in the Foraminifera, when the pseudopodia of two individuals of the same species came into contact they fused and united with one another if the nuclei of the two animals were in the same condition, but when one individual was multinucleate and the other in the condition of having only one nucleus, or if one was undergoing nuclear division and the other in the resting stage, thenno fusion of the pseudopodia took place. Observations of this kind indicate that the nucleus in some way controls the metabolic activities of the cell, and it probably also plays an important part in determining the various modifications undergone by the cells of the different kinds of tissues, though it perhaps has not the exclusive rôle in this respect which has sometimes been ascribed to it. Some account of the evidence with regard to the function of the nucleus in the transmission of hereditary characters will be given in a later chapter.

Before passing on to consider the structure of the nucleus, it may be appropriate to mention a theory which has played a considerable part in cytological investigations-the theory of R. Hertwig that in any species the volume of the nucleus bears a fixed ratio to the volume of the cytoplasm dependent on it. This theory, known as that of the "karyo-plasmatic ratio," or often by its German name of "Kernplasmarelation," originated from the study of the Protozoa, but has received considerable support from observations on 


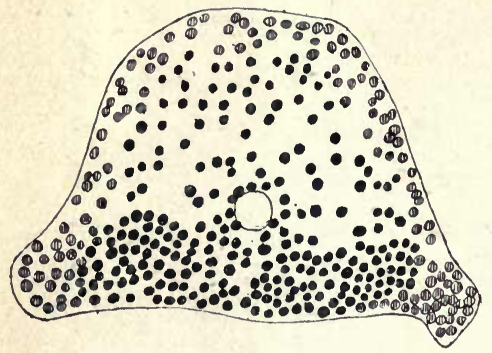

A

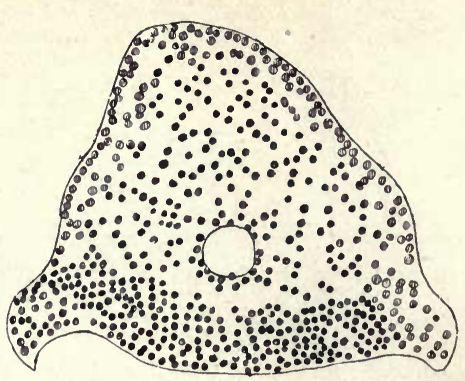

B
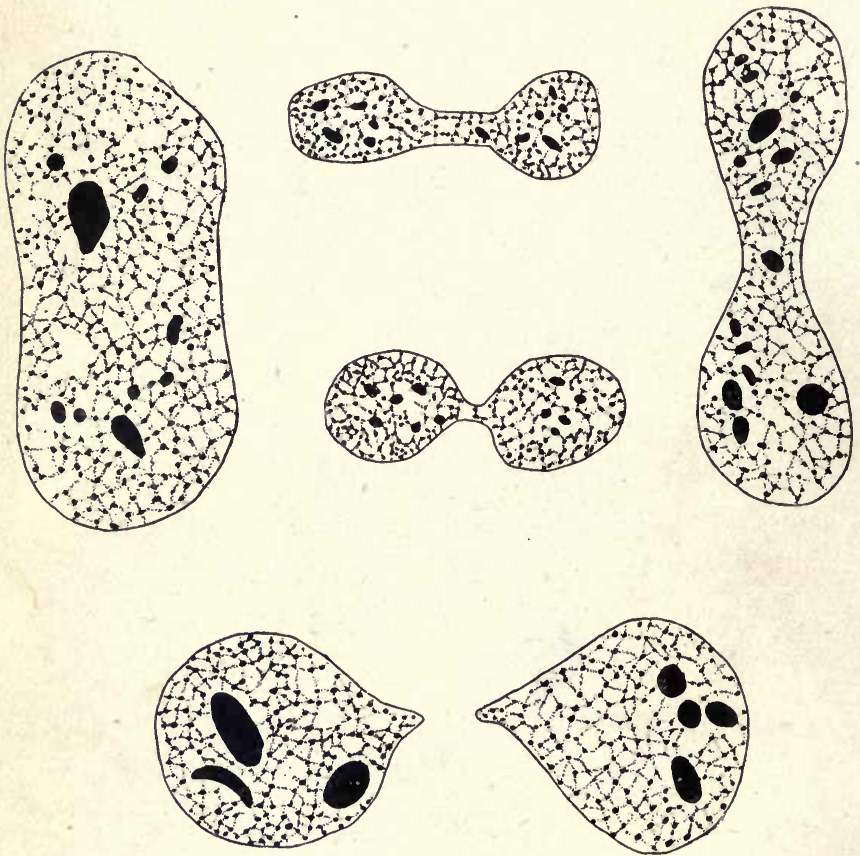

Above-Young Echinus larvae, after Boveri (1903a).

$A$. Larva reared from nucleated egg-fragment in which the egg nucleus united with a sperm nucleus. $B$. Larva reared from non-nucleated eggfragment, from the same parents; the nuclei in $B$ are thus derived from the sperm nucleus only, and are half as large and twice as numerous as in $A$.

Below-Stages of Amitctic division in nuclei of the Malpighian tubules of the Stick-Insect Diapheromera, from Marshall. 
other animals. That it is not universally valid has now been made quite clear, for measurements of the nuclei and cells in different parts of one embryo may show easily recognised differences in the ratio of nuclear to cytoplasmic volume, even before any conspicuous differentiation into tissues has appeared ${ }^{1}$. But that some such relation exists in cells which are homologous, that is, of similar origin and function, is shown by such observations as those of Boveri (1903a) on nuclear and cell size in Sea-urchin embryos. A Sea-urchin egg can be made to develop when unfertilised, and when it does so its nucleus is only half the size of that in the fertilised egg, since the latter is formed by the union of two nuclei, derived respectively from the egg and spermatozoon (PI. I, $A, B)$. When the cells of young larvae derived from fertilised and unfertilised eggs are compared, it is found that in an area of given size there are twice as many cells in a - larva from an unfertilised egg as there are in a like area in a larva from a fertilised egg. That is to say, in larvae from unfertilised eggs both cells and nuclei are half as large and twice as numerous as they are in those from fertilised eggs, with the result that the volume relation between nucleus and cytoplasm remains constant although the nuclei are twice as large in the one case as in the other.

Hertwig was led to formulate his law of the karyo-plasmatic or nucleo-cytoplasmic ratio from observations on the growth and division of Protozoa. In these animals also it has been shown that the law is not universally valid, for under different conditions of temperature and other environmental circumstances the ratio may vary, but under

1 This has been shown especially by ConkLin in the endodermic cells of the Mollusc Crepidula, in which he finds great differences in the Kernplasma-relation in different blastomeres, and in the same blastomere at different stages, depending largely on the rate of cell division, of which it is an effect rather than a cause. 
constant conditions there does seem to be a normal ratio for each species, and causes which seriously upset the ratio lead to the death of the animal. For example, in forms such as Dileptus, in which the nucleus is fragmented, or in Actinosphaerium, in which there are many small nuclei, when the organism is subjected to prolonged starvation the cytoplasm becomes reduced, but the karyo-plasmatic ratio is preserved by the disappearance of some of the nuclei or nuclear fragments, and such organisms can survive very prolonged starvation. But in Paramoecium, in which there is one large nucleus, when the animal is starved the cytoplasm shrinks but the nucleus seems unable to undergo a corresponding diminution, and in such forms starvation rapidly results in death.

Hertwig regards a disturbance of the normal karyoplasmatic ratio as the immediate cause of cell-division, and supposes that the unequal rate of growth of nucleus and cytoplasm brings about a condition of "karyo-plasmatic strain" leading to cell-division and a consequent restoration of the normal ratio. This process has been investigated in detail by Popoff (1908-9), who finds that the period of strain occurs some time before division, since the ratio immediately before and immediately after division is the same. In certain Ciliate Protozoa, for example, after division the cytoplasm increases in volume more quickly than the nucleus until a condition of strain is produced, after which the nucleus increases very rapidly until another division occurs. If, before the strained condition is reached, a portion of the cytoplasm is cut off, division is delayed until the lost portion is restored by further growth; but if the condition of strain has been reached before the piece is cut off, the necessary stimulus to division has been given, and the organism divides in spite of the fact that the excess of 
cytoplasmic over nuclear volume has been removed. It seems, therefore, that although the law of the constant ratio between nuclear and cytoplasmic volume is by no means universal, this ratio has considerable importance in the life of the cell, and is perhaps one of the factors which brings about cell-division.

A typical nucleus consists of a closed membrane enclosing a more or less obvious network of threads, the meshes of which are occupied by a fluid. Observations such as those of HARDY have thrown some doubt on the existence of the network in the nucleus of the living cell, but in some cases at least a real network has been described as visible in living nuclei, and other reasons will be given later for believing that a true structure exists in life. The network is usually described as consisting of a substance called linin, which stains with acid stains; while along the strands, and especially at the nodes of the meshes, there are more or less conspicuous granules of the substance known as chromatin, from the fact that it stains more easily and deeply than the other constituents of the nucleus (Greek cbroma, colour). It is from the chromatin that the bodies called chromosomes, which play a great part in nuclear division, are formed. With regard to the word linin there is a difference of usage among different observers. Some use the term for the oxyphil substance of which the threads of the network seem to be chiefly composed, but others maintain that this substance really consists of oxyphil granules embedded in a nonstaining ground-work, and that it is to the latter that the term linin should properly be applied. HARDY found that it was possible to fix nuclei in such a way that no network appeared, but merely an assemblage of granules, some of which took basic and others acid stains; of these the basiphil granules are doubtless chromatin and the oxyphil probably

D. c. 
the same as the similarly stained granules to which the term linin is sometimes given.

The nuclear constituent to which the greatest attention has been directed is the chromatin, the amount and appearance of which varies greatly according to the condition of the nucleus. In the "resting" condition, that is, when the nucleus has not yet begun to show any signs of approaching division, the chromatin is finely divided, and in fixed preparations scattered in the meshes of the network. Its apparent quantity in this condition is often very much less than when nuclear division is approaching, and there is reason for believing that the granules of chromatin, and the oxyphil granules which make up the greater part of the "linin" threads, are not very different in chemical constitution, and that the latter may be converted into chromatin as division approaches, thus increasing its amount. The behaviour of the nucleus before and during division will be considered more fully in the next chapter.

In addition to the network, with its granules, two other constituents are commonly, though not always, present in the resting nucleus. These are sometimes spoken of collectively as nucleoli, but are of two distinct kinds, known as chromatin nucleoli or karyosomes and true nucleoli or plasMost frequently there is only one of each kind, but two or more may be present, and either one or both may be absent. The karyosome or chromatin nucleolus is a mass of chromatin of varying size, usually round or oval but sometimes less regular in shape. It appears to serve as a reservoir of chromatin from which the chromosomes (see below) may draw part at least of their supply when nuclear division is approaching. In other cases the chromatin nucleolus represents a single chromosome which remains compact during the "resting" phase of the nucleus while the 
other chromosomes have given rise to the nuclear network, as will be explained in a later chapter. The true nucleolus or plasmosome differs from a karyosome in staining with acid rather than basic dyes, and thus consists of plastin rather than chromatin. It is a very conspicuous object in some nuclei, for example, in those of most eggs; it usually has a regular rounded outline, and may be vacuolated. It probably serves as a reservoir of nutritive material, for in Protozoa it has been seen to wax and wane according to the good or poor nutrition of the animal. When the nucleus divides the nucleolus usually breaks up and is absorbed, or portions pass irregularly into one or other of the daughter cells. Although it is usual to separate karyosomes and plasmosomes rather sharply, on the ground that one consists of chromatin and the other of plastin, it nevertheless may happen that a nucleolus contains both substances, usually in the form of a plasmosome in which are embedded masses or globules of chromatin. As nuclear division approaches the chromatin may increase in amount, and it seems probable that the plastin of the nucleolus is converted in these cases into chromatin, which is then transferred to the chromosomes.

The last chief constituent of the typical nucleus is the membrane, about the nature of which there has been considerable divergence of opinion. It is a very definite structure in the resting stage, and is easily seen both in fixed preparations and in the living cell. It usually stains with basic stains, but when exceptionally thick it may also have an outer oxyphil layer. When the nucleus is about to divide the membrane disappears, apparently by a process of solution, and it appears to be formed again from or by the activity of the chromosomes when the daughter nuclei are reconstituted, but opinions differ as to how this process 
takes place. The subject will be further mentioned at a later stage.

Although it is no part of the purpose of this book to deal with the problems of cell-chemistry, it may be appropriate to devote a short space to the chemical constitution of the various parts of the nucleus and of the cell in general. Protoplasm, in the wide sense of the word, consists of a mixture of proteid or albuminous substances of complex chemical constitution, consisting of carbon, hydrogen, oxygen, nitrogen and a small amount of sulphur, phosphorus and usually iron. The cytoplasm is characterised especially by albuminous substances containing little or no phosphorus, while the nucleus contains a relatively large amount of this element, especially in the chromatin. Chromatin is a compound of a substance known as nucleinic acid, which contains up to ro per cent. of phosphorus, with an albumin which is without phosphorus, or in some conditions it is possible that chromatin may consist of uncombined nucleinic acid. The non-chromatic granules and plasmosomes of the nucleus have a larger proportion of albumin and less nucleinic acid, so that they are relatively poor in phosphorus as compared with chromatin.

These facts give some clue to the staining reactions of the various parts of the cell, and also to the changes undergone by the nucleus in its various stages of growth. Nucleinic acid combines with basic stains, whence the affinity of these stains for chromatin, while acid stains are taken up especially by the parts of the cell rich in albumin and poor in nucleinic acid, such as the plasmosome and oxyphil granules of the nucleus, and the cytoplasm. During its various phases the staining reactions of the nucleus vary very largely, and this is probably due to the greater or less amount of combination of albumin with the nucleinic acid 
of the chromatin. In the "resting" nucleus the chromatin stains very faintly as compared with the chromosomes in a nucleus which is approaching division, probably because at that stage the chromatin is combined with a relatively high proportion of albumin, which separates from it as the nucleus prepares to divide. And the conversion of the oxyphil substances in the nucleus into chromatin may similarly take place by the separation from them of the nucleinic acid which is one of their constituents.

Since not only nucleinic acid and its compounds stain with basic stains, but also other organic acids and their compounds, it is not safe to conclude that any basiphil body consists of chromatin. In suitably preserved material all parts of the cell rich in nucleinic acid will take the stain, but bodies containing other acids may do so also, so that other tests may be necessary before concluding definitely that any particular object consists of chromatin.

Apart from the nucleus, there are certain other structures which are so frequently present in cells of the most various. kinds that they are often regarded as typical cell organs. Of these perhaps the most important is the centrosome with its associated structures. These are bodies which play a preponderant part in the mechanism of cell division, and as the next two chapters will be largely devoted to them, they need not be considered further here. Reference must be made, however, to two other groups of bodies which are now known to be of very general if not universal occurrence in cells, and are of a more enigmatical nature. The first of these consists of the structures grouped together under the general name of mitochondria, known also as chondriosomes, or, when rod-shaped, as chondrioconts. These were first described in germ cells which are developing into spermatoz.oa, but recent investigation has shown that they are very 
wide-spread, occurring in cells of many kinds, especially in embryos but also in adult animals. Their nature and behaviour will be described more fully in the chapter on spermatogenesis, but in consequence of the great morphological and physiological importance that has been ascribed to them by some writers, some reference to them is required here. They consist of granules, rods or sometimes vesicles which occur usually in a more or less compact group in the neighbourhood of the nucleus. The extent to which they

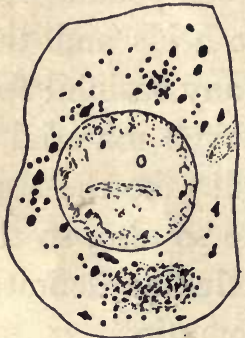

a
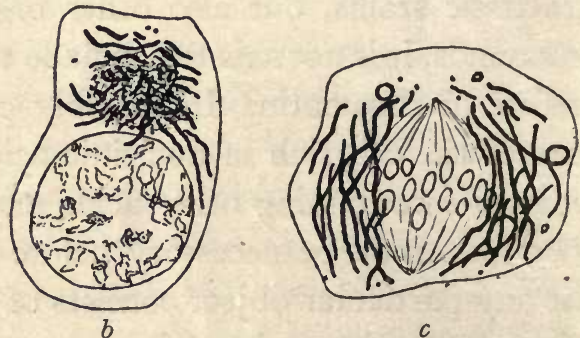

FIG. 1. Mitochondria in the spermatogenesis of Blaps, after DUESBERG ( 1910$)$.

a. Spermatogonium with scattered granular mitochondria.

b. Spermatocyte; mitochondria becoming rod-shaped.

c. First spermatocyte division, mitochondrial rods becoming drawn out around spindle.

are developed varies largely not only in cells of different kinds, but also in the same cell in different stages of its growth. When the cell divides, the mifochondrial bodies are either separated into the two daughter cells, or, according to some observers, undergo division so that the parts are divided into the two cells, The study of mitochondria is less easy than that of the nuclear constituents of the cell from the fact that they are injured or destroyed by many of the fixatives commonly employed, especially those containing acetic acid, and they also require careful staining 
by special methods. They have been observed, however, in the living cell. About their nature and function there has been much dispute. Some investigators maintain that they are permanent cell organs, and that every mitochondrial body arises only from a pre-existing one, and writers of this school have supposed that they play a great part in the transmission of hereditary characters. Others consider that they are comparatively transient structures, coming into existence under special metabolic conditions of the cell, as for example when it is building up or secreting some product of protoplasmic activity such as yolk or skeletal spicules, or when the cell is being rapidly metamorphosed as in the change of a primitive germ-cell into a spermatozoon. In the present state of our knowledge this latter opinion seems on the whole the more probable, but the subject is still very far from being settled. And the fact that mitochondria, like the "Golgibodies," next to be mentioned, have the power of division, and possibly never come into existence except from a pre-existing body of the same kind, certainly supports the opinion held by some cytologists, that they are permanent cell-organs in some ways com- parable to the plastids (chloroplasts, etc.) of plant cells.

Another constituent of the cytoplasm which recent work has shown quite possibly to exist in all cells, and which is certainly present in cells of very varied types from animals of many groups, is the so-called "internal reticular apparatus" of GolGI, often known shortly as the Golgi apparatus. This was first observed in nerve-cells, but more recent investigation has shown that it is so wide-spread that it is quite probably a constant cell-constituent. It is, however, comparatively little known, from the fact that it is invisible in the fresh cell and stains only by very special methods, the chief of which is Golgi's silver impregnation method; it 
may also be made visible by prolonged treatment with osmic acid. It consists either of a network or a system of rods in the neighbourhood of the nucleus, but associated especially with the dense protoplasm known as archoplasm which surrounds the centrosome. In epithelial cells the network seems constantly to lie, in the side of the cell towards the free surface of the epithelium. In nuclear division the network becomes broken up into its component rods, and these are distributed between the two daughter cells and
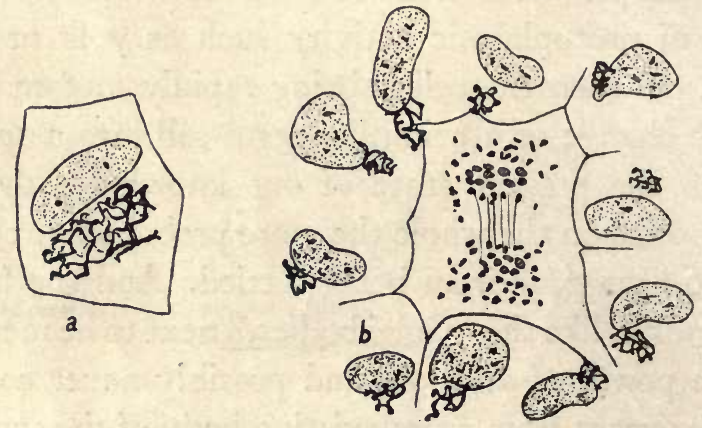

FIG. 2. Golgi apparatus in epidermal cells, after DEINECKa.

a. Golgi network beside the nucleus in cell of horse.

b. Skin of cat, with Golgi network beside the nuclei of resting cells, and broken into small rods around the mitotic figure in the large central cell.

again become arranged around the centrosomes. In some cells the Golgi elements may take the form of granules ("dictyosomes") which are recognisable by their staining reactions, and which multiply by division like the mitochondrial bodies; and in some eggs in which Golgi granules, mitochondrial bodies, and yolk-spherules are mingled together, they may be centrifuged apart into layers distinguishable by their colour, or may even exist in distinct regions in the normal egg. About the true nature and func- 
tion of the Golgi apparatus hardly anything is known; some further reference to it will be made later in connection with the development of spermatozoa.

Finally, a few words must be devoted to the so-called cbromidia observed in certain cells. It has been maintained that these are particles or strands of chromatin emitted from the nucleus, and their existence gave rise to the hypothesis that the nucleus contains two kinds of chromatin, "idiochromatin" concerned especially with reproduction and heredity, and "trophochromatin" with metabolism, an idea based on the extrusion of "chromidia" from the reproductive nucleus of many Protozoa and the existence of separate mega- and micro-nuclei in the Ciliates ${ }^{1}$. The chromidial bodies of certain Metazoan cells were thus supposed to be trophochromatin discharged from the nucleus in order to perform its metabolic functions in the cytoplasm. That bodies which stain like chromatin occur not uncommonly in the cytoplasm is undoubted, but their origin from the nucleus is by no means certain, and their true nature is still obscure. Some of the so-called chromidial bodies are also quite probably in reality mitochondrial rather than chromatinic in origin.

1 See C. C. Dobell (1 g०g). 


\section{CHAPTER III}

\section{Cell Division}

$\mathbb{W} Y^{\mathrm{E}}$ have seen that when a relatively undifferentiated cell grows to a certain size it divides, possibly in consequence of a disturbance in the normal volume-ratio between nucleus and cytoplasm. The division of the cell is always preceded by division of the nucleus, and this may take place in two ways. By far the commoner method of nuclear division is the rather complicated process known as karyokinesis, or mitosis, but in many of the Protoz,oa and occasionally in the Metazoa direct or amitotic division of the nucleus occurs. It will be convenient to consider amitotic division first.

In a cell which divides amitotically the nucleus elongates, becomes constricted in the middle so as to assume a somewhat dumb-bell-shaped form, and then the two halves separate and move apart from each other, after which the cytoplasm becomes similarly constricted and divides between the two nuclei, giving rise to two daughter cells (Pl. I). In a Protozoon the whole series of events can be watched in the living organism, and no doubt can arise with regard to the successive stages of the process. A closely similar series of stages has frequently been described in sections of the tissues of various Metazoa (e.g. the tapeworm, Moniezia (CHILD), various insects, the epididymis of the mouse (JoRDAN)) and there can be no doubt that in some cases, and possibly quite frequently, Metazoan cells divide in this manner. It is sometimes said that in the higher animals 
amitotic nuclear division only occurs in cells which are becoming rather highly differentiated, and which are losing their power of division and approaching a condition of degeneration, but according to CHILD and others amitotic division may occur in such cells as those of the testis from which germ-cells will be produced. It should be mentioned, however, that other observers have not always succeeded in confirming these observations. And it must always be remembered that in tissues satisfactory observations on amitotic division are by no means easy to make. The tissue must be fixed. sectioned and stained, and the occurrence of amitotic division inferred from the series of figures observed. In a number of instances there is no reason to doubt the correctness of the inference; nuclei in all stages of division are found and no other interpretation seems possible. But in other cases it is not easy to decide, in interpreting the figures, whether what is observed is a process of nuclear division or of nuclear fusion, for if, as may happen in certain rather abnormal cases, nuclei come together and then fuse into one, the series of stages in a fixed preparation may be hardly distinguishable from those seen in amitotic division. It may also happen that in some tissues badly preserved mitotic division figures have the appearance of amitosis. Although, therefore, the process is well known in the Protozoa, and certainly occurs here and there in the Metazoa, in the latter animals it has been only insufficiently investigated, and at the present time it is hardly known how frequently it occurs or whether it is to be regarded as normal or abnormal.

In all the higher animals and plants the common and regular method of nuclear division is that known as mitotic or karyokinetic, or sometimes as indirect, division (Pl. II). It is a complicated process, involving a complete transformation 
2. of the nucleus, the disappearance of the nuclear membrane and internal network, the aggregation of the chromatin into chromosomes, and the division of these into halves from which finally two daughter nuclei are reconstituted. The first change that appears in the nucleus when division is approaching is that the chromatin. instead of being scattered in fine granules over the network, begins to become aggregated into larger masses, and at the same time it seems to increase in amount, either in some cases by transference of material from the karyosome, or, possibly, as was mentioned above, by the production of fresh chromatin from the oxyphil (plastin) granules. The concentration of the chromatin may take place in either of two ways; it may become aggregated into irregular masses on the nuclear network, often just beneath the membrane, or the network may resolve itself into a coiled thread or skein known as a spireme, on which the chromatin granules are collected and gradually grow into more concentrated masses. In the first case after a time it is seen that there is a definite number of centres about which the chromatin is being collected; the masses become more compact, including all the previously scattered granules, and finally take the form of rounded, oval or rod-shaped bodies of chromatin known as chromosomes. The number of these is perfectly definite and, with certain exceptions to be considered later, is constant, not only for every cell in the individual animal, but for the whole species. The number differs, however, very greatly in different species, ranging from very few (four, six, or rarely even only two) up to numbers approaching two hundred, but the majority of animals have between twelve and fifty. For reasons which will become clear later the number of chromosomes is commonly an even one. In the second method of concentration, when a skein or spireme is 
formed, the chromatin becomes aggregated upon the thread until it appears either continuous or often divided into small masses like a string of beads ("chromomeres"), and not rarely these granules may be seen to be double, indicating the beginning of a longitudinal split along the thread; the thread then contracts and thickens and becomes segmented transversely into a series of short pieces, which are often loop-shaped. These portions are then chromosomes, and their subsequent behaviour is the same as when they have arisen separately from their first appearance. The two apparently distinct modes by which the chromosomes emerge from the nuclear network differ only superficially, depending, no doubt, simply on whether the clear separation takes place early or later, and even when the spireme appears to have the form of a continuous thread it is quite probable that the chromosomes are really distinct. In their final form chromosomes produced by segmentation of a spireme are often, but not always, elongated and loopshaped, while those formed by concentration around separate centres are usually shorter.

During the later stages of the formation of the chromosomes other important changes have been in progress, leading to the formation of the "mitotic spindle" or mechanism by which the chromosomes after division are separated into the daughter nuclei. During the earlier stages of preparation for division a small granule, which stains deeply with chromatin stains, appears outside the nuclear membrane. This granule is called the centrosome or centriole $\mathbf{1}$, about the origin and nature of which there has been considerable controversy. The centrosome is at first

1 There is some confusion in the use of these words, which will be further discussed in the next chapter, where the nature of the centrosome and mitotic spindle will be considered. 
single, and may appear as a mere stained granule just outside the nucleus, or may be surrounded by a zoné of protoplasm differing somewhat from the remaining cytoplasm of the cell. As the chromosomes are being formed inside the nucleus, the centrosome divides; the halves separate and travel to opposite poles of the nucleus. Here again two somewhat different types of behaviour may be found in different cases. Sometimes, as the daughter-centrosomes separate, a sheaf of protoplasmic threads is seen between them, forming a spindle-shaped structure and converging on the centrosomes at either end, the whole being outside the nucleus. In this case, as the centrosomes diverge, that part of the nuclear membrane nearest to the sheaf or spindle of threads disappears, with the result that when the centrosomes have reached points at opposite sides or poles of the nucleus, the threads pass completely across the nucleus, which is now represented only by a group of chromosomes, the membrane having entirely disappeared.

In the second type the centrosomes pass to opposite poles of the nucleus before any dissolution of the nuclear membrane takes place, and in this case no continuous spindle of threads is formed until rather later (Pl. II, $A, B, C$ ). When the centrosomes reach the opposite poles, the nuclear membrane gradually dissolves, and as it does so a sheaf of fibres is formed across it from centrosome to centrosome. In this second case, therefore, the spindle-fibres seem to be formed at least partially from within the nucleus, while in the former they were entirely extra-nuclear in origin.

At the same time that the spindle-fibres connecting the two centrosomes appear, radiating strands or fibres are also seen running out as rays from the centrosomes into the surrounding cytoplasm. These rays are often quite similar to the spindle fibres except that they taper off and disappear 

in the surrounding protoplasm, instead of being continuous from one centrosome to the other; in other cases they are thinner and less definite. The centrosome with its system of rays is called an aster, and the two asters with the sheaf of fibres connecting them are the achromatic or mitotic spindle.

When the nuclear membrane has disappeared, and the spindle has been formed, the chromosomes are at first scattered irregularly in its neighbourhood, but they soon move to the equatorial or widest region of the spindle, where they arrange themselves in a circle across it (Pl. II, D). This arrangement is very definite, the chromosomes all being in one plane perpendicular to the axis of the spindle or line joining the two centrosomes, and at this stage the chromosomes are said to form an equatorial plate. The details of their arrangement differ according to their number and shape, as will be described below. In the equatorial plate stage it can sometimes be seen that to each chromosome two threads or spindle-fibres have become attached, one from each pole of the spindle, while other fibres run continuously from pole to pole between the chromosomes, and in some animals these fibres are said to be of different thickness, those attached to chromosomes being thicker and called "mantle fibres" (from their appearance at a later stage), while those that run between the chromosomes are thinner and called "interzonal fibres."

When the chromosomes have arranged themselves across the spindle in the equatorial plate, they next split longitudinally into two similar halves, in such a way that a spindle-fibre from one pole is attached to one half, and one from the other pole to the other. Very frequently this longitudinal split can be seen at a much earlier stage, especially in those nuclei in which a spireme is formed; often before 
the nuclear membrane disappears the chromosomes show clear evidence of a split, but commonly as the chromosomes shorten and thicken it becomes obscured, and is not recognisable in the early equatorial plate stage. Now, however, the split reappears, and each chromosome becomes accurately divided into two longitudinal halves. The halves then separate, and, possibly by the contraction of the spindle-fibres attached to them, travel towards the centrosomes or poles of the spindle, one half of each chromosome going towards each pole (Pl. II, $E, F)$. Each daughter group of chromosomes thus contains the same number as was present in the equatorial plate. As the chromosomes travel to the poles, the interzonal spindle-fibres are seen still running continuously from pole to pole, and as the chromosomes approach the poles these interzonal fibres often become somewhat thickened in the equatorial region of the spindle. When the chromosomes get near the poles of the spindle they become crowded together, and often change their form; in some cases they become swollen and vacuolated, each looking like a minute nucleus, in others they appear to send out branches which begin to interlace with others of neighbouring chromosomes. Gradually from this crowded mass, by further swelling and coalescence of the chromosomes, a nucleus is formed, and at the same time the interzonal spindle fibres and the rays of the asters become indistinct, and the cytoplasm of the cell begins to divide, the line of division running exactly across the plane of the equator of the spindle (Pl. II, $F, G$; Text-fig. 4, p. 38).

Put quite shortly, then, the main stages of mitotic nuclear division are as follows: First, the chromatin of the nucleus becomes aggregated either in isolated masses or in a spireme, in preparation for the formation of chromosomes, and at the same time the centrosome appears, divides into 
two, and these begin to separate and the beginnings of the achromatic spindle and astral rays appear. The next phase is the movement of the centrosomes to opposite poles of the nucleus, the formation of definite chromosomes, and the disappearance of the nuclear membrane with the concurrent completion of the spindle and asters. These two stages are spoken of as the prophase of mitosis, the first being the early, and the second the late prophase. The chromosomes then take up their definite position in a circle in a plane transverse to the line joining the centrosomes; this is the equatorial plate stage. The chromosomes divide, so that there is now a circle of double chromosomes in the equatorial plate, constituting the metaphase, which seems to last for a short time before the halves of the chromosomes begin to diverge towards the poles of the spindle. The chromosomes then begin to travel along the spindle towards the centrosomes, one longitudinal half of each chromosome going to each pole; this stage is known as the anaphase. Finally, the chromosomes become crowded together at the poles and coalesce to form the daughter-nuclei, and the cytoplasm of the cell begins to divide across the middle of the spindle; this last stage of division is the telophase. Since the same process takes place in every. division of every cell from the fertilised egg onwards (with the exception of the developing germ-cells and certain abnormal divisions, which will be mentioned later) and since in each division the chromosomes are accurately halved by a longitudinal split, it follows that every cell of the body contains the same number of chromosomes. And since, as will be seen later, there is reason to believe that the longitudinal splitting divides each chromosome into similar halves, it follows also that each cell contains a complement of similar chromosomes.

D. C. 
A short general account of the process of cell-division has now been given, and it remains to discuss in rather fuller detail some points connected with the behaviour of the chromosomes. The questions involved in the formation of the chromosomes from the scattered chromatin of the resting nucleus will be considered later; here it will be convenient to deal with the arrangement of the chromosomes on the spindle, and the manner of their division and separation to form the two daughter-nuclei.

When the nuclear membrane has disappeared and the chromosomes are fully formed, their shape varies greatly in different species of animals, and in some cases there is considerable variety among the chromosomes in any one cell. Where there is such diversity, it is often possible to recognise individual chromosomes, or rather corresponding chromosomes, in each mitotic figure, and to see that the same series reappears at every cell-division; and in a hybrid between two distinct species the chromosomes derived from each parent species may be recognised by their characteristic form. In general the chromosomes have the form of rods of nearly uniform thickness and with rounded ends, but they vary in length from rods so short that they are practically spheres, to elongated bodies whose length is twenty or more times their width. It has been maintained ${ }^{1}$ that the width of all the chromosomes of any one species, and even any group, of animals, is constant, and although this is almost certainly not true of different kinds of cells, it is generally found that all the chromosomes in anyone mitotic figure have approximately the same width, but may differ in length and in the shapes into which they are bent. Short chromosomes are commonly straight, but the larger ones are usually bent in the form of a $\mathbf{V}$, of which the two

$$
{ }^{1} \text { E.g. by MEEK (1912). }
$$


arms may be equal in length, or one may be shorter than the other, giving a hook-shaped form. The characteristic shape is best seen in the equatorial plate and early anaphase stages; in the prophase the bending is often irregular, and at that stage the chromosomes can only be identified by their relative lengths.

When the chromosomes take up their position in the equatorial plate, their arrangement depends both on their
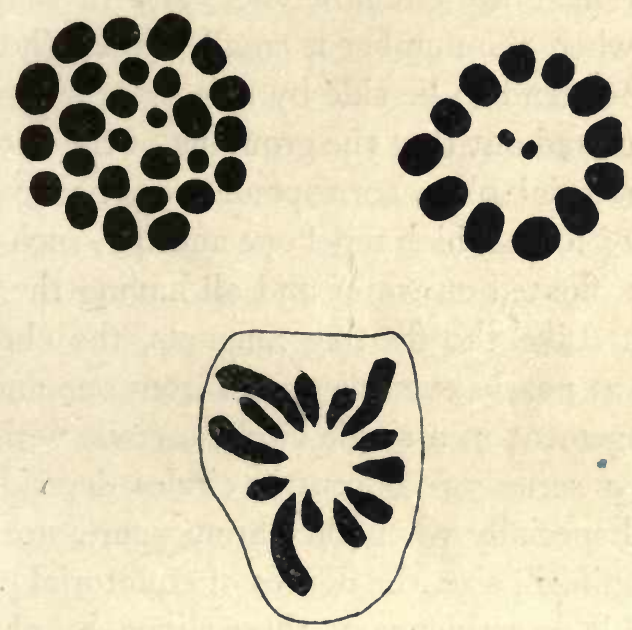

FIG. 3. Examples of equatorial plates showing characteristic arrangement of chromosomes. From PAYNE (r 909 ) and Robertson (1915). Compare also Figs. 24, 29 and Pls. XVII, XVIII.

number and on their shapes. Long, rod-shaped chromosomes are typically arranged in a radial position, with their inner ends converging in the centre of the equatorial plate, their outer ends towards the circumference. Chromosomes bent into the form of a $\mathbf{V}$ have the angle pointing towards the centre and the two arms diverging outwards (cf. Fig. 29, p. 233). Very short rods and nearly spherical chromosomes are commonly arranged in circles, at even distances from one 
another; if their number is small there may be one circle, with or without a chromosome in the centre; when the number is larger there are two or more irregular concentric circles. Where the chromosomes of one group differ in size, there is no constant arrangement, though it often happens that similar groupings recur repeatedly in different equatorial plates; for example, if there is one very small chromosome it often takes up a central position, while larger ones are usually near the circumference, and in some species, especially when the number is small, similar (homologous) chromosomes tend to lie side by side or near together. It has been pointed out that the grouping of the chromosomes in the equatorial plate corresponds very nearly with that of centres of force which repel one another, such as a series of magnets floated on water and all having the same pole uppermost. Like the floating magnets, the chromosomes are placed at nearly even distances from one another, and their arrangement in a single circle, a circle with a central group, or a series of concentric circles depends on their number. Especially when the chromosomes are short and of nearly uniform size, the figures of equatorial plates have a remarkable resemblance to those given by physicists of the groupings of various numbers of magnets floating in a confined space, and this fact may have some bearing on the theories concerning the mechanism of nuclear division which will be briefly discussed later.

When the chromosomes begin to divide in the metaphase, their behaviour again depends on their shape. In the equatorial plate the long axis of each chromosome is perpendicular to the axis of the spindle, but in anaphase the chromosome lies lengthways on the spindle, parallel with the spindle-fibres. In the case of long, rod-shaped chromosomes, when the longitudinal split is completely formed, the 
inner or central ends of the two halves diverge first, while the two half-chromosomes remain in contact at their outer ends, and complete separation does not take place until the two halves have rotated apart until almost in line with each òther. The spindle-fibres are attached to the diverging ends, and give the appearance of drawing these ends apart by their contraction, while the opposite ends of the two halfchromosomes remain in contact. The halves of $\mathbf{V}$-shaped chromosomes separate first at the angle of the $\mathbf{V}$, and the tips of the arms are the last portions to separate, so that in early anaphase the dividing chromosome has a diamond or lozenge shape. The spindle-fibres are attached to $\mathbf{V}$-shaped or hook-shaped chromosomes at the point of the bend.

Very frequently all the chromosomes divide and the halves travel to the poles quite simultaneously, so that the two "daughter-plates" are very regular; this is so especially when the chromosomes are of about uniform size. In other figures some chromosomes divide and diverge before the others, with the result that in anaphase they are scattered rather irregularly over the spindle, some approaching the poles before others have left the equatorial region. Sometimes one or more chromosomes constantly divide before or after the remainder. These irregularities are most frequent in the so-called maturation divisions of the developing germ-cells; simultaneous division, with chromosomes very evenly disposed in anaphase, is the common condition in ordinary "somatic" cells.

The formation of the daughter-nuclei from the groups of chromosomes at the poles of the spindle in the telophase is difficult to observe in detail because of the crowding of the chromosomes. When, as often happens, the individual chromosomes in the late anaphase become vacuolated and take the form of small vesicles, the nucleus appears to 
result from the coalescence of these vesicles, and its membrane to be formed from their outer walls, much as a number of small soap-bubbles may combine to form one large bubble. It is more probable, however, that the membrane is formed by some interaction between the substance of the chromosomes and the surrounding cytoplasm, and is of the nature of a precipitate which is re-dissolved when the nucleus is again ready to divide. There are reasons, which will be considered in a later chapter, for believing that at least the

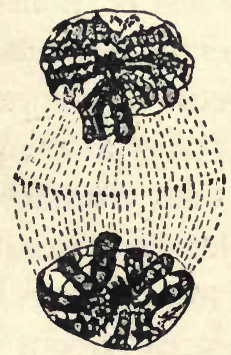

FIG. 4. Telophase of second maturation division of the pollen of Paris quadrifolia, after BoLles LEE (1912). The nuclear membrane is already formed around the parts of the nuclei away from the spindle, imperfect next to the spindle. The chromosomes are vacuolated, attached by their ends to the nuclear membrane, and connected with one another by transverse branches.

framework of the chromosomes persists in the "resting" nucleus, and that they are in fact permanent entities which remain from one cell-division to the next. That the membrane is not always formed by the coalescence of vacuolated chromosomes is shown by the fact that in the telophase of

- mitosis the compact chromosomes may often be seen within a large vesicular nucleus.

Finally, before passing on to consider the nature of the "achromatic spindle," there are a few points which can conveniently be mentioned at this stage. These concern 
especially the position of the nucleus in the cell, and the relation of nuclear division to the division of the cytoplasm which normally follows it.

Since the plane in which the cell divides is perpendicular to the axis of the spindle, it follows that the position of the spindle determines the plane of cell-division, and the position of the spindle in turn depends on that of the nucleus. Generally speaking, at least in embryonic cells or those which are not highly differentiated, the nucleus occupies a position which may be regarded as the centre of gravity of the cytoplasm. A spherical cell in which the cytoplasm is uniformly distributed has the nucleus at the centre; but if there are considerable masses of other substances present, for example yolk, and if, as often happens, these are arranged in zones so that the greater part of the cytoplasm is at one pole and the rest of the cell is largely occupied with yolk, then the nucleus lies at the centre of mass of the cytoplasm independently of the yolk or other inclusions. When a cell of this kind is about to divide, the spindle is formed in such a way that its axis lies along that of the greatest mass of cytoplasm, with the result that in general when the cell-division takes place the cytoplasm is about equally divided between the two daughter cells. This happens irrespectively of the yolk or other inert substance, so that a cell may divide into two very unequal parts, one containing little except cytoplasm and the other a similar amount of cytoplasm and a large amount of yolk, as regularly takes place in the third cleavage division of a yolkladen egg like that of the Frog. The way in which the mass of the cytoplasm influences the position of the spindle and the consequent plane of cell-division is illustrated by the results of compressing yolk-laden eggs with glass plates. If the egg, with the cytoplasmic pole uppermost and the yolk 
below, is compressed between horizontal plates, not only the first and second, but also the third segmentation division will be in a vertical plane, because the cytoplasm is extended horizontally by the pressure and the spindles will therefore lie horizontally. If, however, the egg is compressed vertically by being sucked into a tube of diameter narrower

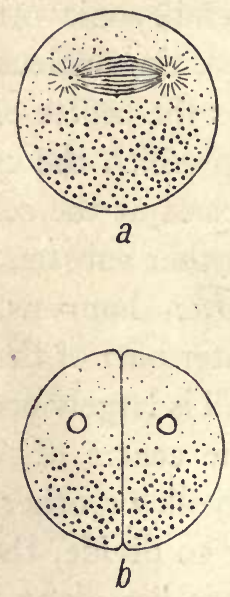

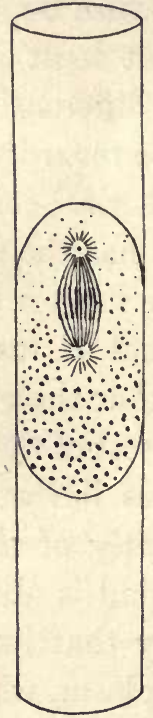

C

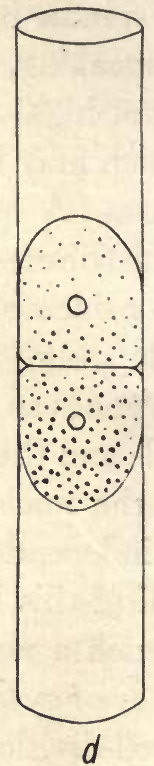

FIG. 5. Diagram of eggs segmenting normally and compressed in glass tubes.

$a, b$. Normal first segmentation division.

$c, d$. First division horizontal instead of vertical, in consequence of pressure. (After Przibram.)

than that of the egg, the second segmentation division will be in a horizontal plane, instead of the normal vertical one, in consequence of the vertical extension of the cytoplasm which causes the spindles to be vertical. The very unequal cell-division which takes place in the separation of the polar bodies from the egg seems to have a different cause, and will be considered in a later chapter. 
The actual division of the cytoplasm is very probably due to diminished surface tension around the equator of the cell at the greatest distance from the poles of the spindle ${ }^{1}$. It can easily be shown by experiment that when the surface tension of a sphere of liquid is reduced around its equator, it will tend to divide along the equatorial plane. The relation of the formation of the division-furrow which initiates cytoplasmic division to the position and size of the spindle has been stated thus: the speed with which the appearance of the cytoplasmic division-furrow follows the metaphase of the mitosis and the depth to which it extends (in meroblastic eggs) are in general proportional to the length of the spindle. That is to say, the shorter the spindle, the nearer it must be to the surface of the cell if the furrow is to be formed in a given time; or if the spindle has a given length, the deeper it lies below the surface the longer it will take to produce a division-furrow. Herlant has shown that, other things being equal, the length of the spindle is greater if there is more nuclear matter, so that indirectly the rate at which cytoplasmic division occurs varies with the size of the nucleus if the cytoplasmic volume is constant.

Generally speaking, any bodies included in the cell, apart from the nucleus, seem to be distributed purely mechanically with the cytoplasm into the two halves of the cell as it divides. A possible exception to this rule is found in the mitochondria, to which a short reference has already been made, but it will be convenient to postpone any further account of them to the chapter on the development of the germ-cells.

1 R. S. LILLIE ( 1916$)$, from experiments with segmenting eggs placed in dilute sea water, concludes that the surface tension of the cell is increased around the poles while remaining constant at the equator. This is of course equivalent to a relative diminution around the equator. 


\section{CHAPTER IV}

\section{The Centrosomes, Spindle and Asters}

$A^{\text {LTHOUGH }}$ the behaviour of the chromosomes in Al nuclear division has especially attracted the attention of cytologists, in consequence of the probability that in it is to be found the solution of some of the fundamental problems of heredity, yet the nature and mechanism of the achromatic spindle offers problems hardly less fascinating, and perhaps equally far-reaching as regards the nature of vital processes. From the short account of nuclear division given in the preceding chapter, it will be seen that the process consists in the accurate longitudinal division of the chromosomes, which are the essential part of the nucleus, and the separation of the halves into the two daughter cells. The mechanism by which this process is carried out is the achromatic spindle with the centrosomes at its poles, and although the spindle is such a familiar object, there has been, and still is, the utmost diversity of opinion about its nature and origin.

The spindle arises as a sheaf of fibres between the two centrosomes formed by the division of the original centrosome which appears shortly before the nucleus divides. With regard to the origin of the centrosome itself there is no complete agreement. When cells are rapidly dividing, there is no doubt that the centrosome may persist from one cell-generation to the next; the daughter-nucleus, after a division, is formed close to the centrosome at the pole of the spindle, this centrosome then divides, a new spindle begins to be formed between the halves at right angles to the axis 
of the old spindle, and a fresh nuclear division commences. But, as will be seen shortly, the centrosome frequently undergoes considerable changes during the progress of a nuclear division, and in these cases it is not certain that the centrosome of one nuclear division is identical with that of the preceding, though it may be formed from it. The opinion, therefore, that the centrosome is a permanent cell-organ, as constant as the nucleus, is of very doubtful validity, and in cells which are not dividing often no trace of such an organ may be found.

In some cells, however, the centrosome certainly persists as a permanent organ, for in certain flagellate cells, such as the collar-cells of sponges and the bodies of some of the Flagellate Protozoa, the centrosome is the basal granule of the flagellum, and the same is true of the mother-cells of spermatozoa, in which the axial filament of the "tail" grows out from the centrosome. In many epithelial cells also the centrosome can constantly be seen as a single or double granule.

Those who hold that the centrosome is in some sense a permanent organ regularly existing in every cell regard it as arising originally from the "middle piece" of the spermatozoon which fertilised the egg from which the animal originated. There is no doubt that the middle piece of the spermatozoon is at least partly formed from the centrosome of the cell which gives rise to the spermatozoon, and that after fertilisation the centrosomes of the first segmentation division of the egg arise either from or in connection with the middle piece (see Chapter VIII). But as will be seen below there is no certainty that the centrosomes of the first division are actually produced by division of the middle piece, and it seems equally possible that they originate from the egg-cytoplasm in consequence of some chemical change 
induced by the middle piece of the spermatozoon, or possibly in some cases by the nuclear substance of its head. That the egg-centrosomes do not always have such a source is proved by their presence in the segmentation divisions of parthenogenetic eggs (eggswhich develop unfertilised). Some observers have maintained that in this case the centrosomes arise from those of the last (maturation) division of the eggnucleus, but others have described the origin of centrosomes de novo in non-nucleated fragments of eggs under the influence of various chemical reagents, and at the present time, although the question is by no means completely settled, the evidence tends to show that centrosomes can arise afresh from the cytoplasm, and that they are temporary structures produced by some chemical change concomitant with nuclear division, and are not in any true sense invariable constituents of the cell. This conclusion is supported by the fact that true centrosomes appear to be absent in the higher plants, in which the process of mitotic division is otherwise similar to that in animals.

Considerable confusion has been introduced into the literature of the subject by the rather different senses in which the word centrosome has been used. At an early stage, before the spindle is completely formed, the centrosome usually has the form of a small deeply staining granule surrounded by a rather dense mass of protoplasm. Some investigators use the word centrosome for the whole structure, and give the name centriole for the central granule; others call the granule a centrosome, and speak of the surrounding mass as the centrosphere (or astrosphere) ${ }^{\mathbf{1}}$. It

1 Strictly, the word astrosphere was originally used for the structure surrounding the centrosome, and centrosphere for the whole body-centrosome and astrosphere. The older term "attraction-sphere" is equivalent to astrosphere. 
is probably most convenient to use the former terminology - to designate the whole structure as a centrosome and to use the word centriole for the central granule when it is present. Using the words, then, in this sense, it is found that the centrosome shows considerable variation in form, not only in different species of animals, but also in the different stages of one cell-division. Not rarely a centrosome, which at first has the form of a small rather dense mass with central granule, swells up, as the nuclear division proceeds, and becomes greatly enlarged; the granule may become vacuolated and disappear, and the whole body take the form of a considerable mass of vacuolated protoplasm from the edges of which the astral rays and at one point the spindle-fibres radiate ( $\mathrm{cf}$. Pl. II). Then a fresh concentration may take place in the centre, and a new granule or centriole may appear within it, and if the cell is undergoing repeated rapid division this new centriole may divide in the anaphase or telophase of one nuclear division in preparation for the next division which is to follow it. Sometimes the whole organ has a concentric structure, a central granule enclosed in dense protoplasm, and outside this a clear space, which is in turn surrounded by a more dense zone from which the astral rays arise; or some of these rays may originate from the central mass and cross the intervening clear space, while the remainder radiate from a zone nearer the circumference.

From the preceding account it will be seen that both the origin of the centrosome and its relation to the asters and spindle of the mitotic figure are still somewhat uncertain. There can be no doubt that in the first segmentation division of many eggs the centrosomes arise in connection with the middle piece of the spermatozoon, but that this is not the only possible method of origin is proved by the 
existence of exactly similar centrosomes in eggs which develop parthenogenetically. It is usually said that in the fertilised egg the middle piece of the spermatozoon becomes separated from the "head," and that while the head swells up to form the male pronucleus the middle piece divides, giving rise to the two centrosomes of the mitotic spindle. This is certainly the simplest interpretation of the figures commonly seen in fertilisation, but detailed observation of the process in certain cases suggests that it may not be a complete account of the process ${ }^{1}$. In the Axolotl, for example, Jenkinson (1904) found that the middle piece of the spermatozoon, after separating from the "head," swells up and becomes a vacuolated mass or sphere, around which an aster begins to develop, apparently by means of fluid travelling out of the sphere and pushing out elongated alveoli in a radiating manner around it (P1. III). As the sphere enlarges by becoming more and more vacuolated, more distant radiations appear in the surrounding protoplasm, which JENKINSON attributes to the attraction of fluid from the egg-substance into the swelling sphere. Within the sphere a more solid body now appears, which is the definitive centrosome; this divides, the two halves separate, and a spindle is formed between them, lying between, and to some extent including, the egg- and sperm-nuclei, which are now of equal size and nearly in contact. The nuclear membranes then disappear, the chromosomes come to lie on the spindle, and the mitotic figure of the first segmentation division is definitely established. In the equatorial plate stage the centrosomes then swell and become vacuolated and soon

1 That the middle piece of the spermatozoon is not completely converted into centrosomes is proved by the discovery of Meves that in Echinoids a portion of the middle piece remains in the egg-cytoplasm during segmentation and can be traced as far as the 32-cell stage (1912, 1914). 

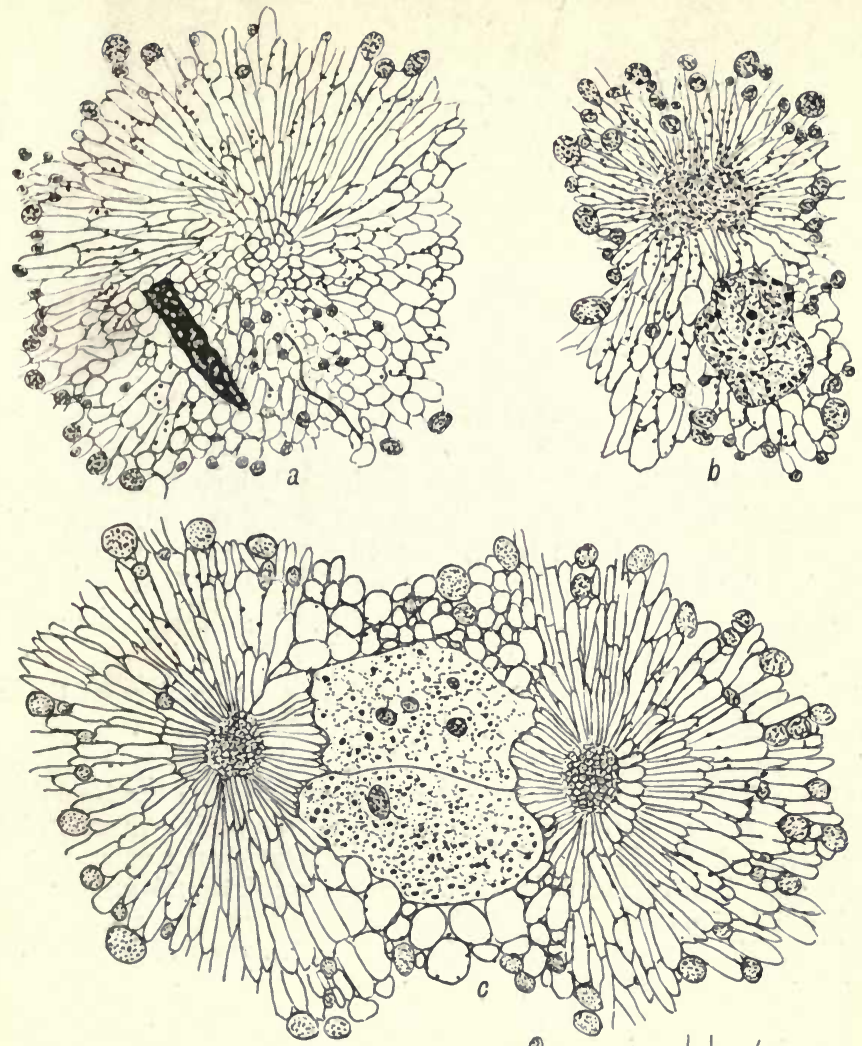

1. o.

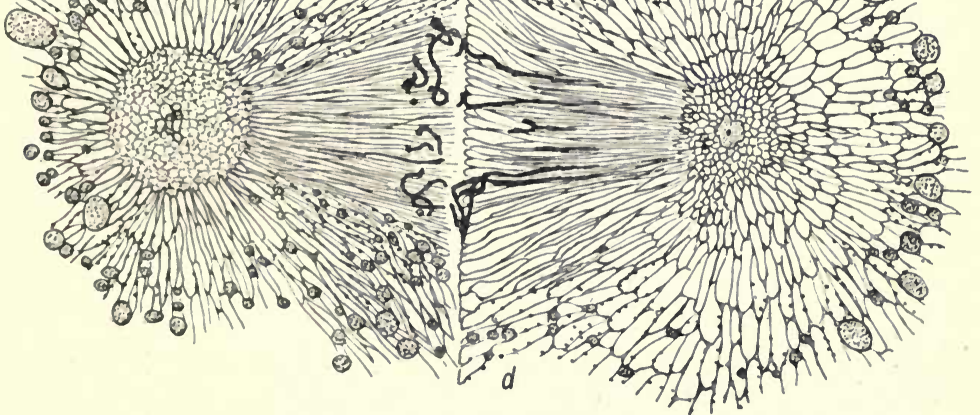


Explanation of Plate iII.

Stages in the fertilisation of the egg of the Axolotl, after JENKInson.

a. The sperm head (becoming vacuolated) and part of the tail. The middlepiece has disappeared and is represented by vacuolated protoplasm with radiating alveoli, on the walls of which are pigment granules.

b. The sperm head has swollen to form a nucleus. A centrosome has appeared in the centre of the alveolar radiations, and is dividing.

c. The daughter-centrosomes have separated to opposite sides of the conjugating egg and sperm nuclei.

d. First segmentation division. Equatorial plate stage represented on the left, early anaphase on the right. On the left, a centrosphere has been formed by reticular degeneration of the inner rays, and within it a vacuolated centrosome: Spindle of irregular fibres with pigment on the outer rays. On the right, a homogeneous centrosome has appeared, containing a centriole which divides in the late anaphase in preparation for the succeeding nuclear division. (Redrawn from Quart. Journ. Micr. Sci. Xlvili. Pls. 30, 32, 33.) 
merge altogether in the spheres, but in anaphase a new small centrosome containing a central granule appears in each sphere, and in the telophase the granule or centriole divides in preparation for forming the centrosomes of the next succeeding division.

JENKINSON interprets theseobservations thus. The middle piece of the spermatozoon dissolves producing a centre of high osmotic tension to which water is drawn from the surrounding cytoplasm. This gives rise to the vacuolation and outgrowth of radiating alveoli, and the path of the water travelling from every side towards this centre causes the outer radiations. Then nucleinic acid from the head of the spermatozoon (sperm-nucleus) causes a precipitation within the vacuolated sphere, and this is the definitive centrosome. In the later stages of division a somewhat similar process recurs, giving rise in the telophase to the centrosomes which will act as poles of the spindle in the next division. The bearing of these observations on the formation of the spindle itself will be considered later; here it may be noticed that the formation of a centrosome by precipitation at the centre of a system of rays or alveoli is just what has been described as taking place in unfertilised eggs of various sorts under the influence of chemical reagents, and if JENKINSON's observations may be accepted as typical, they seem to provide a middle way between the two divergent views-that centrosomes always arise from pre-existing centrosomes, or that they may be formed de novo in the cytoplasm. In corroboration of JENKINSON's interpretation of the origin of the centrosome it should be mentioned that according to F. R. Lillie (I9I9) the middle piece of the spermatozoon of Nereis is thrown off with the tail and the aster and centrosome arise in connection with the nuclear substance of the head. 
With regard to the nature and origin of the astral rays and spindle-fibres opinions vary no less than in respect of the centrosomes. The many hypotheses that have been put forward may be classed in three main groups:

(I) A considerable number of observers maintain that + the asters and spindle are merely modifications of a reticular or alveolar structúre existing in the cytoplasm generallythat the threads of the reticulum or walls of the alveoli take on a radial arrangement around the centrosomes, and that where the rays from the two centres meet one another they unite and form the spindle.

(2) Boveri (1888), on the contrary, formed the opinion that the whole mitotic figure is formed from a special kind of protoplasm, named by him archoplasm, which in the resting cell exists around the centrosome and from which, when nuclear division approaches, the asters and spindle are produced. Boveri's archoplasm hypothesis has been adopted in a somewhat modified form by many subsequent writers, who maintain that while the fibres of the asters and spindle differ in nature from the general protoplasm of the cell, yet the whole substance from which the mitotic figure is formed is not present in the resting cell as such; they regard the fibres as growing out from the neighbourhood of the centrosome and being formed from the general cytoplasm as they grow, much as crystals may grow in a saturated solution. In this sense the fibres would be formed by differentiation from the cytoplasm, but would not be due to a merely local rearrangement of its structure.

(3) A third group of cytologists believe that the spindle and asters are simply the expression of lines of force radiating from the centrosomes, and causing the particles of the cytoplasm to arrange themselves in lines or chains as iron-filings do under the influence of a magnetic field. 
Some have maintained that the force in question is actually electric; others, for example, HaRTog, regard it as a force peculiar to the living cell.

In the space available only a very brief statement of the arguments for and against each of these hypotheses can be given. Most of those who accept the view that all protoplasm has a reticular or alveolar structure also hold that the mitotic spindle consists of a local rearrangement of the fibres or alveoli, giving rise to the spindle and astral fibres, and this opinion has also been supported by some who do not take any decided view on the structure of protoplasm in general. For example JenKinson, in the work on the egg of the Axolotl already referred to, concluded that the asters and spindle were due to alveoli radiating from centres into which fluid was attracted owing to their osmotic condition. According to him, the asters in this case consist of radiating alveoli surrounded by a zone of finer rays which are due to the path of the fluid travelling towards the centres; the spindle is formed by the meeting of elongated alveoli from the two centres, in such a way that continuous tubes are formed from one centre to the other, and the fibres are produced by the partial coalescence of neighbouring tubes, the walls of which give rise to viscous threads. These threads become attached to the chromosomes, and when the chromosomes divide, the halves are drawn towards the centres or poles of the spindle by the contractility of the viscid threads. Many other investigators have given somewhat similar accounts of the origin and mechanism of the spindle, differing indeed in detail, but agreeing in the essential features that the spindle consists of the same substance as the rest of the cytoplasm, and that the fibres draw the divided chromosomes towards the poles by their own contractility. This is essentially the view associated

D. C. 
with the names of Bütschli, Flemming, van Beneden, Heidenhain, not to mention many more recent workers.

The second, or archoplasm hypothesis of BovERI, and its more recent modifications, are supported by the fact that in many cases, especially when the surrounding protoplasm has an alveolar structure or contains yolk, the rays from the asters can be seen growing from the centres in the walls of the alveoli or between the yolk-granules, and are obviously distinct from the protoplasm in which they lie. They appear more dense, stain more deeply, and give the impression of consisting of a different substance. Moreover, at the close of mitosis, when the cell divides, the remains of the spindle and asters may contract into a mass which often has a somewhat fibrous structure and which persists for some time. This mass is often known by its German name of Spindelrestkörper, and by some writers as spindle-bridge, and is sometimes spoken of as the Nebenkern, but this latter is an unfortunate word, since it has been applied to bodies of quite distinct nature and origin, and has often been used for the "mitochondrial" body which will be described at a later stage. The fact, however, that in some cases at least not only are the astral rays and spindle-fibres apparently quite distinct from the surrounding protoplasm, but also that their remains persist for a considerable period after cell-division is completed, is rather strongly in favour of the view that they are not mere rearrangements of the ordinary cytoplasmic structure, but are composed of a somewhat distinct substance. It is possible, of course, that the truth is represented by a compromise between these

- two hypotheses - that the asters and spindle-fibres are formed by a modification of the general cytoplasm, and that the extent of this modification varies in different cases, so that in some cells the fibres consist of protoplasm hardly 
differing from that composing the rest of the cell, while in others considerable differentiation takes place, giving rise to dense, deeply stained threads which seem to support the idea of a special archoplasm. This intermediate view is supported by the fact that during the later stages of mitosis, when the astral rays and spindle-fibres still persist, the centrioles may divide and begin to form new asters around them in preparation for the next division, which are quite distinct from the persistent rays of the division that is still in progress. Such a formation of new rays is not consistent with the view that the rays and fibres are mere re-arrangements of a fibrous structure already existing, but at the same time it is hard to reconcile with the archoplasm . hypothesis in its original form, for if all or nearly all of the archoplasm were used up in the old system of rays, it is difficult to understand from what source the new rays are derived. If, however, the rays and fibres are formed as a temporary modification of the cytoplasm by the action of some force acting in the centrosomes, the difficulty would seem to be overcome.

The two views so far considered agree in regarding the spindle and astral rays as consisting of fibres, probably of a viscid or semifluid consistency, and in supposing that when the spindle is fully formed, its fibres, or at least the outer "mantle-fibres," are attached to the chromosomes, and draw the halves apart towards the two poles by their contractility when the chromosomes divide. Sharply contrasted with this view is the hypothesis of which HARTOG is the chief exponent, which regards the whole structure as due to the production of lines of force radiating from the centrosomes. HARTOG speaks of the rays and spindle-fibres as "chains of force," that is to say, chains of particles drawn into a linear arrangement by an attractive force just as iron-filings 
are drawn into lines radiating from the pole of a magnet.

- By ingenious experiments Hartoc has imitated the mitotic spindle, not only in one plane by filings on paper between north and south poles of a magnet, but also in three dimensions by suspending the filings in a viscous fluid. He points out that in order to get lines of force connecting the two poles, it is necessary that the poles should be unlike (positive and negative), and he maintains that all the phenomena of nuclear division are explicable on the assumption of forces of unlike sign acting at the centrosomes. Since none of the ordinary known forms of force is applicable he assumes a force peculiar to the dividing cell which he calls mitokinetism. He regards the movement of the halved chromosomes towards the poles of the spindle as due to attraction by this force, so that on this view the fibres do not draw the chromosomes to the poles, but merely act as guides along which they move. Others, for example Gallardo, have maintained that the force is electric, and that both the poles have the same sign. This involves the assumption that the spindle-fibres do not run from one centrosome to the other, but from the centrosomes to the chromosomes in the equatorial plate; the chromosomes are supposed to bear a negative charge, and the centrosomes a positive. Although there is evidence that the chromosomes are electrically negative as compared with the cytoplasm, GALLARDo's suggestion is open to the fatal objection that spindle-fibres may undoubtedly run from pole to pole apart from the chromosomes, so that this opinion may be dismissed ${ }^{1}$. Hartog's hypothesis, which rests both on experiment and on careful examination of dividing cells, is less easy to disprove. There are several facts, however, which tell rather strongly against it. (I) In many mitotic figures the spindle-fibres appear to be quite

1 See Note on page $5^{8}$. 
definitely attached to the chromosomes, and as has already been mentioned, the characteristic forms of the chromosomes in anaphase seem partly to depend on the point of attachment of the fibre. (2) The spindle-fibres are sometimes quite definite in number, and so clearly marked that they can be counted in transverse section; this is difficult to understand if they are mere "chains of force." They also may thicken as they shorten during the anaphase, and the apparent complete disappearance of the mantle-fibres in the telophase can be accounted for on the supposition that they consist of a viscous fluid which contracts to a small round drop. (3) The whole mitotic figure ("amphiaster") often shows signs of possessing considerable rigidity; cases have been described in which when the cell was burst the spindle was forced out as a whole into the surrounding fluid, where it could be seen rolling over and over and persisting for some time before it broke up. Also several observers, for example, Morgan (1910), and ConkLin (1917), find that in centrifuged eggs the spindle may be bent and deformed, and can push aside yolk-granules and other dense particles, and that in some cases it may be firmly attached to the peripheral layer of the egg by the astral rays. These observations seem definitely to contradict the chains of force hypothesis, but it must be added that other investigators, for example F. R. Lillie (1909), maintain that the rays are reformed after every change in the position of the poles, and that centrifuging experiments support rather than disprove the chains of force hypothesis. (4) In some cells, such as leucocytes, the asters ate said to persist and to form part of the contractile mechanism of the cell. (5) It seems almost certain that at times the rays from two asters may cross one another, which should be impossible on the hypothesis of chains of force. In view of these and similar facts it seems probable that the supposition of a "dual force" as the cause 
of the mitotic spindle is mistaken, in spite of the remarkable resemblance to spindles of models which can be made by means of unlike magnetic poles. Other investigators have sought to show that the processes of mitosis can be explained by osmotic, and especially by surface-tension, phenomena. Considerations of space forbid any attempt to discuss these ideas in detail, and it must be concluded that, for the present at least, no really satisfactory explanation of the process has been given ${ }^{1}$.

Finally, a short space must be devoted to the division of the cytoplasm as distinct from that of the nucleus. In a normal cell-division, when the two nuclei are reconstituted in the telophase, the cell-body divides in the plane of the equator of the spindle, and if the spindle is in the middle of the cell, the cell is divided into equal halves. When, however, the spindle is excentric, as in the segmentation of a yolk-laden egg, or in the more extreme case of the extrusion of the polar bodies, the cell divides unequally. This would suggest that cell-division is determined by the position of the spindle rather than by the nucleus, and this conclusion is supported by centrifuge experiments, in which the various substances of the egg may undergo all kinds of re-arrangements, while the division plane nevertheless depends on the position of the spindle, or perhaps on that of the asters. If, for example, owing to the centrifuging, the polar spindle of an egg is greatly elongated, an enormously enlarged polar body may result; and in an egg centrifuged during segmentation, the visible substances in the cytoplasm may be completely rearranged without greatly affecting the nature of the subsequent division, so long as

1 For a concise account of some of these hypotheses, and of the physical principles of a bipolar field of force as applied to the dividing cell, the reader is referred to D'A. W. Thомpson's On Growth and Form, pp. 168-190 (Cambridge 1917). 
the mitotic figure retains its normal position. This fact also indicates that the position of the mitotic figure does not depend on the arrangement of the visible substances of the cell, but rather on an invisible polarity which controls not only the orientation of the mitotic figure, but also the normal distribution of the various inclusions in the cytoplasm. In an earlier chapter it was mentioned that the nucleus commonly occupies a position which may be regarded as the centre of mass of the cytoplasm apart from inclusions, and that when the cell divides, the cytoplasm is typically divided into equal parts, with the result that one daughter-cell may be much larger than the other if it contains abundant yolk or other inclusions. The centrifuge experiments suggest that these results depend not on the nucleus but on the position of the mitotic figure; the mitotic figure is orientated according to the polarity of the cell, and by its position determines that of the daughternuclei when the cell divides.

The question then remains what part the mitotic figure plays in the division of the cytoplasm. The cytoplasm typically divides in the plane of the equator of the spindle, which might suggest that the spindle itself is the determining factor. In abnormal eggs of various species, however, "monasters" have been observed; that is to say, owing to the failure of the centrosome to divide, only a single aster is formed, with no definite spindle. In such a case the chromosomes do not split, and are all drawn towards the single centre, but nevertheless a cell-division may follow, a portion of cytoplasm being separated off at the pole of the egg furthest from the centrosome. This production of a "cell" with no nucleus or centrosome formed under either natural or experimental conditions has been described in several instances, and the observations lead to the conclusion that the division of the cytoplasm takes place in the 
plane farthest removed from the influence of the centrosomes'. Several investigators have supposed that celldivision is determined by surface tension, and that division takes place along a zone where the surface tension is reduced. If, then, for any reason the zone of the cell farthest from the centrosomes has a lower surface tension than elsewhere, this would be in accord with all the facts, for in a typical dividing cell the plane of the equator of the spindle is that farthest removed from the centrosomes.

This conclusion is supported by Teichmann's rule of the relation between size of mitotic spindle and formation of division furrows, quoted in the preceding chapter. The rule states that the rate of formation of the cytoplasmic furrow varies with the length of the spindle and with its nearness to the surface of the cell, and if the amount of reduction of surface tension varies with the distance from the centrosome, evidently it would be relatively greater the longer the spindle and the nearer the spindle to the eggsurface. Or, otherwise expressed, if the surface tension of the cell surface is greatest in the region nearest the centrosomes and least in the region farthest away from them, the greatest possible difference will be produced by having the longest possible spindle lying parallel with the surface and as near it as possible.

The greater part of the experimental and observational evidence thus suggests that cell-division is ultimately dependent rather on the mitotic figure, and probably more especially in the position of the centrosomes, than on division of the nucleus which normally precedes it. fact, however, is not at first sight quite easy to recongade

1 That the centrosomes do exert some kind of "influence at a distance" is indicated by the fact that in the synapsis (synizesis) stage of developing germ cells (see Chapter V) the spireme is crowded towards the side of the nucleus nearest to the centrosome, and $\mathrm{HARDY}_{\mathrm{A} D}$ has produced similar figures in resting nuclei by subjecting them to an electric current ( $\left.1913{ }^{1}\right)$. 
with this conclusion, which was mentioned in a former chapter when the size-relations of nucleus and cytoplasm were being considered. In an egg which, in consequence of abnormal conditions, receives two spermatozoa, each may give rise to two centrosomes, so that four centrosomes may exist together, and cause the production of a quadripolar spindle. The result of this is that the combined egg and

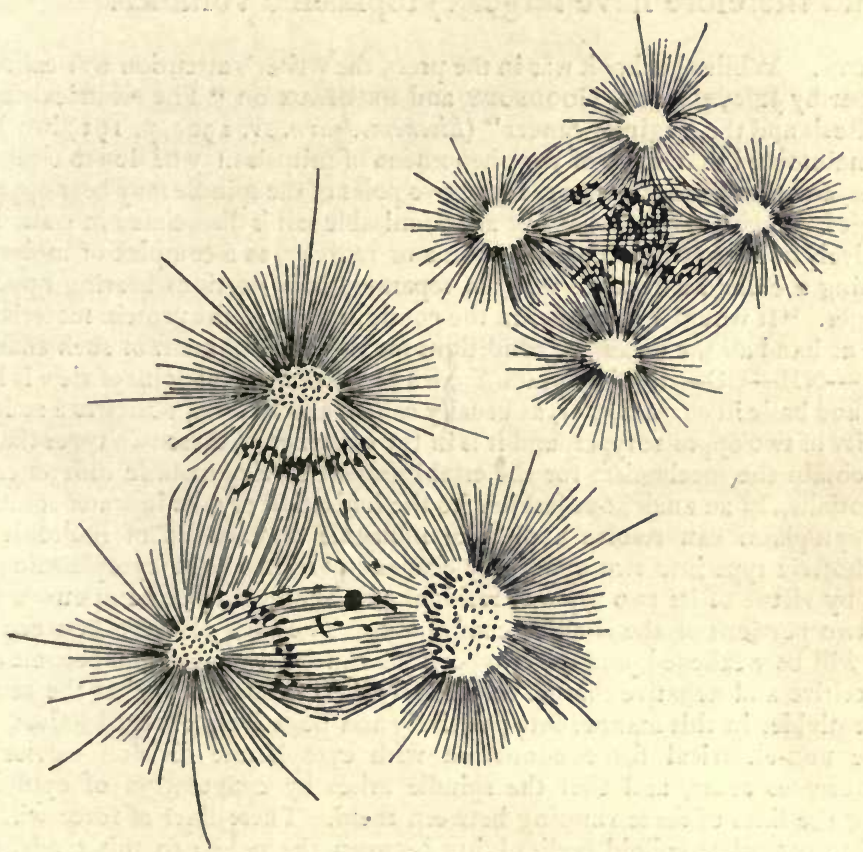

FIG. 6. Tripolar and quadripolar spindles in segmenting Echinoid eggs, after GRAY (1913).

If $\$$ herm nuclei divide simultaneously into four. Usually the $\mathrm{nO}_{\mathrm{ar}}$ daughter-nuclei contain unequal numbers of chromo"Jmes, and in consequence are of different sizes, and it is found that the cell divides in such a way that the volume of cytoplasm around each nucleus is roughly proportional to the volume of the nucleus. In this case, therefore, the plane of division seems to depend primarily on the nucleus 
itself, rather than to be determined solely by the achromatic figure $^{1}$. It is possible, however, that this may be brought into harmony with the conclusion that cytoplasmic division is determined by the spindle and asters by means of HERLANT's observation that the length of the spindle varies with the size of the nucleus. The cells with larger nuclei would thus have larger spindles, and the daughter-cells would therefore have larger cytoplasmic volumes.

Note. While this book was in the press, the writer's attention was called to a paper by Jessup, BALY, GoODBODY and PRIDEAUX on "The electrical forces of mitosis and the origin of cancer" (Biochem. Journ. IV. 1909, p. 191) in which it is maintained that many of the phenomena of mitosis may be due to electrical forces, and that the centrosomes at the two poles of the spindle may bear opposite charges. They point out that just as an ionisable salt is dissociated in water into positively and negatively charged atoms or radicles, so a complex of molecules forming a colloid substance may be separated into portions bearing opposite charges. "It would appear that in the configuration of the protein material we have at hand all the necessary conditions for the establishment of such charges. The - NH - CO - linking which, from a physicochemical point of view is both acid and basic in character, or, as usually called, amphoteric, possesses a residual affinity of two opposite types, and it is in the existence of these two types that we can obtain the mechanism for the establishment of electrostatic differences of potential.... In an analogous fashion [to the ionisation of salts in water solution] the cytoplasm can resolve a discrete molecule or complex of molecules of amphoteric type into two oppositely charged portions. The cytoplasmic mass can, by virtue of its two types of residual affinity, form loose compounds with the two portions of the simpler compound. The lines of force between the two will be weakened, and the two portions can be separated and become seats of positive and negative charges respectively." It is suggested that the centrosome divides in this manner into positively and negatively charged halves, that some non-electrical force connected with cytoplasmic division carries the centrosomes apart, and that the spindle arises by coagulation of cytoplasm along the lines of force running between them. These lines of force will also tend to coagulate colloid bodies lying between the poles; to this tendency is ascribed the condensation of the chromosomes in the nucleus. The condensed chromosomes divide by the action of the same forces which previously caused the division of the centrosome. These split chromosomes would be forced into the equatorial plate of the spindle, and by the attraction of the centrosomes the negatively charged half of each chromosome will travel to the positively charged centrosome, and vice versá. It must be assumed that the charges are such as completely to neutralise one another at the telophase of the division. This hypothesis, though open to several of the objections which apply to HARTOG's "mitokinetism," has the advantage of not postulating any totally unknown force.

1 In this connection see also PAINTER, T. S. (1918). 


\section{CHAPTER V}

\section{The Maturation of the Germ-cells: Spermatogenesis}

$7^{\mathrm{HE}}$ two preceding chapters have dealt with the phenomena of cell-division in ordinary body cells. In these cells the essential feature of nuclear division is the accurate halving of the chromosomes, with the result that each of the two daughter-nuclei contains the same number as the parent nucleus from which they arose, and a corollary from this is that every nucleus of the body (except where an abnormal division has occurred) contains a similar complement of chromosomes. It is evident, however, that at some point a different process must take place, for in almost all animals and in the great majority of plants at some stage in the life-cycle sexual reproduction occurs. The essence of sexual reproduction is the union of two cells derived from different parents, accompanied by the fusion of their nuclei, and when conjugation of nuclei occurs it is clear that the chromosome number of the zygote (product of the union) must be doubled. If, then, the germ-cells of the two parents each contained the number of chromosomes typical of the species, the number in the zygote would be doubled, and, unless there were some provision for halving the number, the doubling would recur in every sexuallyproduced generation.

In all organisms in which sexual reproduction occurs this difficulty is avoided by the process known as the reduction of the chromosomes, which usually takes place in the celldivisions immediately preceding the production of the conjugating cells or gametes, although it may occur (e.g. in 
some Protozoa and in the higher plants) several or many cell-generations earlier. The chromosome reduction is brought about by a nuclear division of a peculiar type, of which the essential feature is that instead of splitting longitudinally as in a normal division, the chromosomes become associated in pairs and then separate entire, one chromosome of each pair going to each pole of the spindle. That this is the true nature of the process is now generally recognised, although there is no universal agreement about the details by which it is effected, and until comparatively recently there was extreme divergence of opinion with regard to the interpretation of the phenomena. Gradually, however, agreement as to the fundamental similarity of apparently different methods is being reached, and the former acute conflict of opinion is being replaced by something approaching unanimity, at least as far as the basal principles are concerned. The process of reduction is most easily studied in the development of the spermatozoa of animals and of the pollen grains of the flowering plants, and as a general introduction to it, it will be convenient to give a somewhat generalised account of spermatogenesis.

In the majority of animals both spermatozoa and ova are descended from cells which appear rather early in development and are known as primitive germ-cells. These cells, usually together with a certain amount of other tissue which becomes associated with them, give origin to the gonads (testes and ovaries), and very frequently they give rise not only to the germ-cells themselves but also to accessory cells of various sorts. As the gonad becomes completely formed, cells derived from the primitive germcells can be distinguished as those from which the actual gametes (spermatozoa or ova) will be produced, and these are known in the male as spermatogonia, in the female as 
oogonia. They divide a number of times by ordinary mitotic division, in which it is seen that they have the ordinary or somatic number of chromosomes. During these divisions there may again be differentiation among the products into
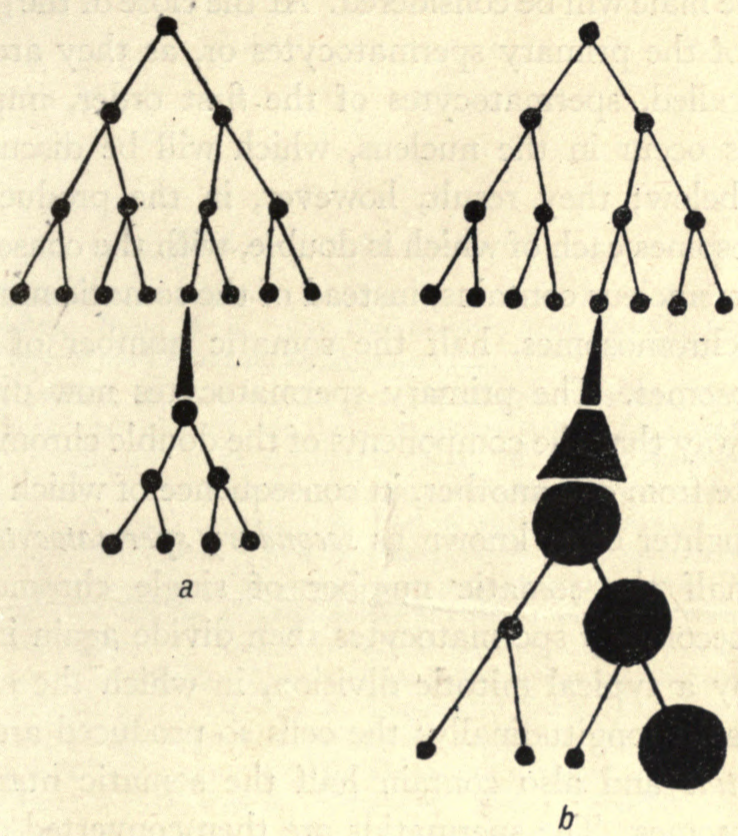

FIG. 7. Diagrams of spermatogenesis (a) and oogenesis (b), after GRÉGOIRE.

Three spermatogonial and oogonial divisions are represented, followed by a growth-phase leading to a primary spermatocyte and oocyte. The growth-phase is at first similar in each sex, but is followed by a second stage in the female not represented in the male. The two successive spermatocyte divisions correspond with the two polar divisions of the egg.

cells which will give rise to gametes and others which perform some accessory function, such as, for example, the supply of nourishment to the gametes. After a number of divisions, which in some animals is definitely fixed and in others probably variable, the spermatogonia and oogonia cease to 
divide and begin to increase considerably in size. At this stage they are called primary spermatocytes in the male and oocytes in the female. From this point onwards the behaviour in the two sexes is different, and for the present only the male will be considered. At the close of the growthphase of the primary spermatocytes or, as they are sometimes called, spermatocytes of the first order, important changes occur in the nucleus, which will be discussed in detail below; they result, however, in the production of chromosomes each of which is double, with the consequence that the nucleus contains, instead of the somatic number of single chromosomes, half the somatic number of double chromosomes. The primary spermatocytes now divide in such a way that the components of the double chromosomes separate from one another, in consequence of which each of the daughter cells, known as secondary spermatocytes, contains half the somatic number of single chromosomes. These secondary spermatocytes then divide again immediately by a typical mitotic division, in which the chromosomes split longitudinally; the cells so produced are called spermatids and also contain half the somatic number of chromosomes. The spermatids are then converted directly into spermatozoa without any further division.

The whole process may be summarised thus. The spermatogonia contain the somatic number of chromosomes; each spermatogonium of the final generation enters on a period of growth and nuclear change and becomes a primary spermatocyte. The primary spermatocyte just beföre division contains half the somatic number of double chromosomes; it divides by a peculiar division giving rise to two secondary spermatocytes which have half the somatic number of single chromosomesw The two secondary spermatocytes so formed divide by a typical division, giving rise to 


\section{Explanation of Plate IV.}

General scheme of the maturation divisions (meiosis) in the spermatogenesis of

Lepidosiren. From W. E. Agar, Quart. Journ. Micr. Soc. Vol. LVII. I 9 I I, p. 9; figures kindly lent by Dr AGAR.)

$A$. Resting nucleus, primary spermatocyte. $B$. Leptotene stage. $C$. Threads beginning to pair side by side (Lepto-zygotene stage). D. Completion of pairing ("bouquet" stage, zygo-pachynema). E. Strepsitene stage, beginning of synizesis. The double threads have split except at their ends, forming long loops. F. Late synizesis. G. End of synizesis. Bivalent loops are shortening and thickening, and are beginning to break up into their constituent univalents. $H$. The nuclear membrane has disappeared. The loops have broken up into the diploid number of chromosomes each with a transverse constriction. I. Appearance of the spindle. Second pairing of the chromosomes beginning. J. Second pairing complete; definitive bivalents taking their place on the spindle. $K$. Anaphase of first spermatocyte division; bivalents separated into univalents each still having a transverse constriction. Spindles rotating in preparation for the second division. $L$. Metaphase of second spermatocyte division. Univalents, still transversely constricted, splitting longitudinally. 
Plate IV

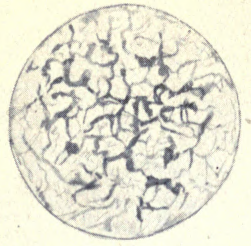

A

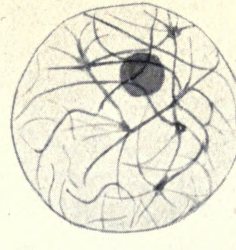

B

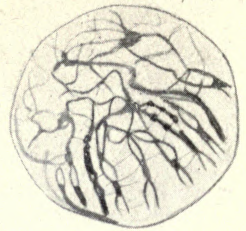

C

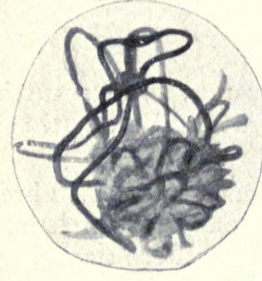

F

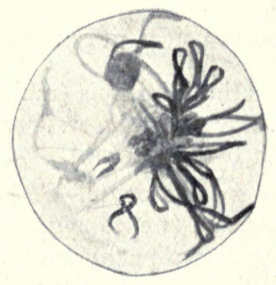

E

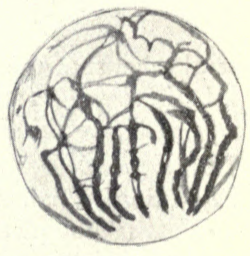

D

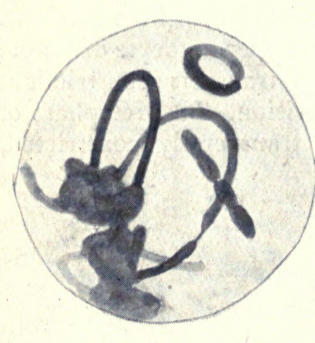

G

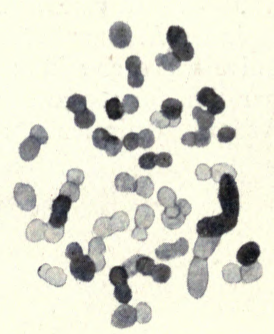

H

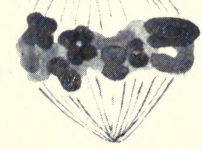

L

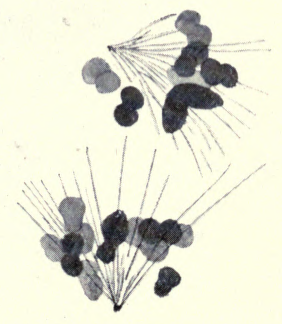

K

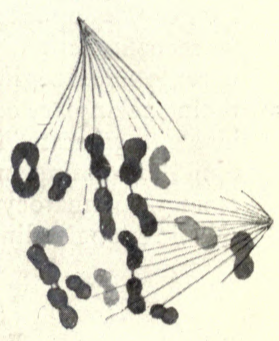

I

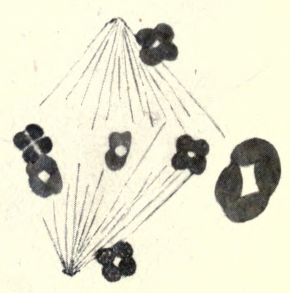

J 
four spermatids, and each spermatid develops into a spermatozoon. Every spermatozoon, therefore, is formed from a cell the nucleus of which contained half the number of chromosomes characteristic of the species.

The whole series of events from the beginning of the growth-phase of the primary spermatocyte to the production of the spermatids is now generally known by the term meiotic phase, introduced by FARMER and MOORE (1905), and it will now be necessary to consider the processes involved in rather greater detail. As there is still considerable divergence of opinion, not only in the interpretation of individual cases, but also on the question whether the phenomena are really comparable in different animals, it will be advisable first to describe one specific case, and a convenient example to choose, both from the excellence of the work and the clearness with which the various stages are seen, is the spermatogenesis of the American Lung-fish, Lepidosiren paradoxa, described by Agar (I9I I) (Pl. IV).

In the spermatogonial nucleus the chromatin is distributed in coarse masses over a dense network of threads which fills the nucleus, and the nucleus of the primary spermatocyte at the end of the growth-phase is somewhat similar, differing chiefly in the chromatin being spread out in threads (Pl. IV, $A$ ). As the division approaches, these threads become finer and more regular and distinct, but have no definite arrangement $(B)$. Then at one pole of the nucleus (in many animals, and probably in Lepidosiren, at the pole nearest to the centrosome) the threads take up a parallel arrangement for part of their course, while remaining as a tangled network at the opposite pole. The parallel parts of the threads now begin to fuse in pairs, often becoming spirally twisted round each other in the process $(C, D)$. In this way thick double threads are produced 
converging towards one pole of the nucleus, and branching out into a network of fine threads which still occupies its distal hemisphere. Gradually the pairing of the threads proceeds further and further, while at the same time they become shorter and thicker, with the result that in a short time the network in the distal hemisphere is entirely unravelled, and the nucleus contains only a number of double threads arranged in long loops or horse-shoes, of which the free ends converge towards the pole where the fusion first began $(E, F)$. The number of these loops is half that of the somatic chromosomes. This stage is often spoken of as the "bouquet" stage, and is followed by a rapid shortening and thickening of all the threads, which become more or less closely tangled together towards the pole of the nucleus in a tight mass from which loops project into the nearly empty distal half $(F, G)$. This contraction and crowding together was originally called synapsis, but as the word has been used in two different senses MCCLUNG's term synizesis is preferable ${ }^{1}$. The contraction and crowding make detailed observation difficult, but from the examination of exceptionally clear nuclei, and from comparison with other animals, AgAR is convinced that the threads which paired in the bouquet stage have now separated again except at their ends, with the result that there are now half the somatic number of elongated, twisted rings, composed of threads in pairs attached to each other at their ends but otherwise single $(G)$. These threads are now much thicker than they were before the parallel conjugation, owing to their

1 As was mentioned earlier (p. 56 note) HARDY has imitated the synizesis stage of spermatogenesis in resting somatic nuclei of plants by passing through them an electric current. The greater part of the chromatin became collected at the side of the nucleus toward the positive pole, with loops extending into the distal half, just as in synizesis the chromatin is contracted towards the pole nearest the centrosome. 
contraction, and from this stage onwards the shortening and thickening proceeds rapidly. At the same time the tight mass which was formed in synizesis becomes loosened and the threads are seen to have contracted into relatively short, thick chromosomes, most of which still have the form of rings, in consequence of their being united by their ends in pairs. In Lepidosiren a process now follows which has not been described in many other animals, but which will possibly be found to be of more general occurrence. The chromosomes as they emerge from the clumping of synizesis are double, consisting of rings made up of two single chromosomes; these rings now break up into their components, giving rise to the somatic number of more orless rod-shaped chromosemes, most of which are contracted in the middle, thus having a somewhat dumb-bell-like shape $(\mathrm{Pl}$ IV,$H)$. In the majority of animals this separation of the rings into their single halves has not been described. As the chromosomes become disentangled from the clump of the synizesis stage, the nuclear membrane dissolves and the spindle begins to be formed; at the time when the membrane disappears the centrosomes have yet not reached opposite sides of the nucleus but lie comparatively near together, and from each a sheaf of fibres runs towards the irregular group of chromosomes. Gradually the centrosomes rotate apart until they come to lie on opposite sides of the chromosome group, and a definite spindle is formed between them. Meanwhile the chromosomes come together again in pairs, and for the second time form double ring-shaped bodies which take up their position in the equatorial plate of the spindle $(I, \mathcal{F})$. Owing to the constriction in the middle of each single chromosome, these rings generally have the appearance of being four-fold, looking like four rounded bodies in contact with one another, but from the manner of their

D. c. 
formation it is quite clear that they are in reality double rather than quadruple, being composed of two rods, somewhat bent and with their ends in contact, and each deeply constricted in the middle. The constriction corresponds with the point to which the spindle-fibre is attached, and which becomes the apex of the $\mathrm{V}$-shaped chromosome of the anaphase stage, and is seen also in ordinary somatic chromosomes in Lepidosiren. The apparently four-fold condition of the chromosomes at this stage is of importance, since it closely resembles the "tetrad" form which at one time was regarded as characteristic of this stage of the developing germ-cell, as will be mentioned below.

The double chromosomes which thus arrange themselves in the equatorial plate of the spindle are in every case composed of two similar halves; the chromosomes of Lepidosiren differ considerably among themselves in size and shape, but each ring is composed of two similar members, and it can hardly be doubted that the two members of each ring are the same two which paired together in the bouquet stage and which composed the rings in the synizesis that followed it. On the equatorial plate they are arranged so that one member of each pair is directed towards one pole of the spindle and the other towards the other pole, and the two members of each pair then begin to separate from each other and diverge towards the poles. They remain connected for a time by two strands of chromatin connecting the ends that were in contact, giving a very characteristic appearance which is typical of the division of the primary spermatocytes, or of the "reducing" division generally, in a large number of animals and plants (Pl. V, $G, H, I)$. A division of this form is known as a beterotype mitosis ${ }^{\mathbf{1}}$, and results in

1 The heterotype or heterotypical form of mitosis was formerly regarded as characteristic of, and confined to, the first maturation division of the 



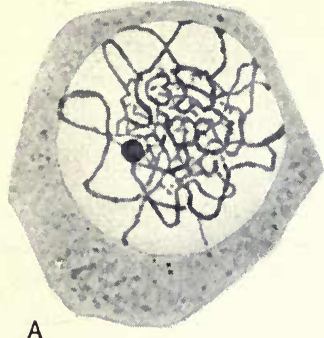

A

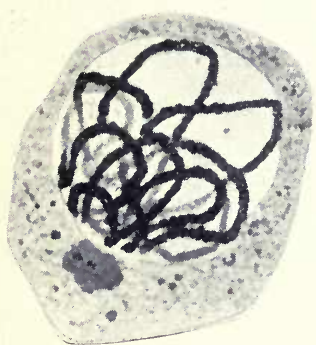

D

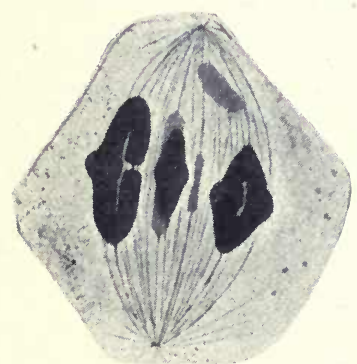

G

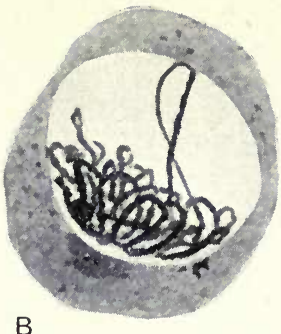

B
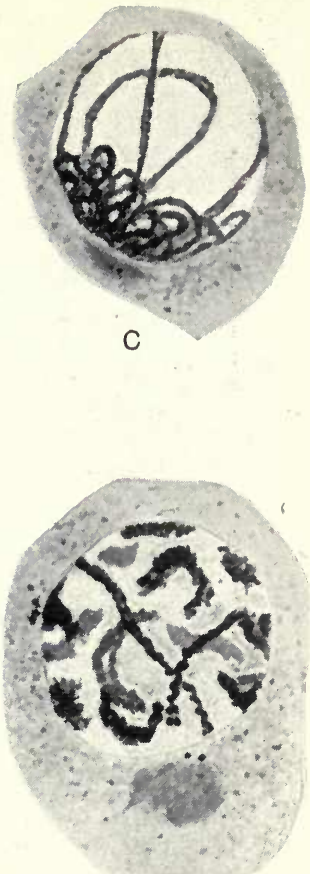

F

$A-F$, prophases of heterotype division of spermatocytes of Guinea-pig, after MoOre and WALKer. $A$, spireme (leptonema), $B$, synizesis (contraction phase), $C$, zygonema, $D$, pachynema, $E$, resolution of contraction phase. $F$, appearance of definite double chromosomes.

$G, H, I$, heterotype division in spermatocytes of the Grasshopper Stenobothrus, after Davis. $G, H$, Metaphase, $I$, Anaphase. 
the production of two daughter-nuclei (secondary spermatocytes), each of which has half the somatic number (and therefore often called the "reduced" number) of single chromosomes. Almost immediately after they are formed, the secondary spermatocytes begin to divide again. This time the chromosomes arrange themselves in the ordinary way in the equatorial plate, split longitudinally, and the halves travel to the poles as in an ordinary division (Pl. IV, $L$ ). This second division is therefore spoken of as a bomotype mitosis, and gives rise to the spermatids, which proceed to develop directly into spermatozoa.

Before proceeding to discuss how far the events here described are typical of the "meiotic phase" in the maturation of the germ-cells of animals and plants in general, it will be convenient to mention certain terms which are now coming into general use to describe the various stages (Pl. V, $A-F)$. The terms (mostly introduced by von WinIWARTER of Liége, 1900) have the advantage of avoiding considerable circumlocution in describing the stages of maturation, but unfortunately alternative names introduced subsequently have caused a certain amount of duplication. The early stage of the primary spermatocyte, when the nucleus contains a network of thin threads, is called leptonema; that when the threads unite in pairs (or in any case, when they first become double) is zygonema; the "bouquet stage" with thick threads is pacbynema, and the succeeding condition in which the thread again appears double or loop-like, interpreted by AGAR as a separation of the paired threads except at their ends, is strepsinema or diplonema. The corresponding adjectives are leptotene, zygotene, pachytene and strepsitene. Also it germ-cells of vertebrates and the higher plants; it is now known in many other groups. 
is sometimes convenient to use MCCLUNG'S term syndesis to describe the union of threads or chromosomes in pairs; the word synapsis is often used in this sense, but as it is also used in the sense of synizesis (contraction of the chromatin-threads into a compact mass), the word synapsis tends to be misleading. The union of chromosomes in sideby-side conjugation is called parasyndesis (parasynapsis), and the end-to-end union (formerly supposed to be frequent or universal) is telosyndesis (or telosynapsis). The condition in which the chromatin is all arranged in looped threads (formerly supposed to be one continuous coiled thread, but now more frequently regarded as a number of intertwined loops) is called the spireme, and the stage at which the spireme by contraction of its loops gives rise to the reduced number of short double chromosomes is diakinesis. The appearance of half the somatic number of double chromosomes in consequence of syndesis is often spoken of as pseudo-reduction.

The course of events in the meiotic or maturation phase of the spermatogenesis of Lepidosiren is on the whole typical of the corresponding stages in a large number of animals and plants. At almost every step of the process, however, there are points of detail, some of them of very great theoretical importance, about which there are differences of opinion. Frequently, no doubt, these disagreements among observers depend on real differences in the behaviour of the nuclei and chromosomes in different animals, for it can hardly be supposed that the phenomena are quite identical in such different groups as, for example, Worms, Insects and Vertebrates. The surprising thing, in fact, is not the existence of differences, but that on the whole they should be so small, and that closely similar phenomena should be found in all the higher animals and plants. In many points, 


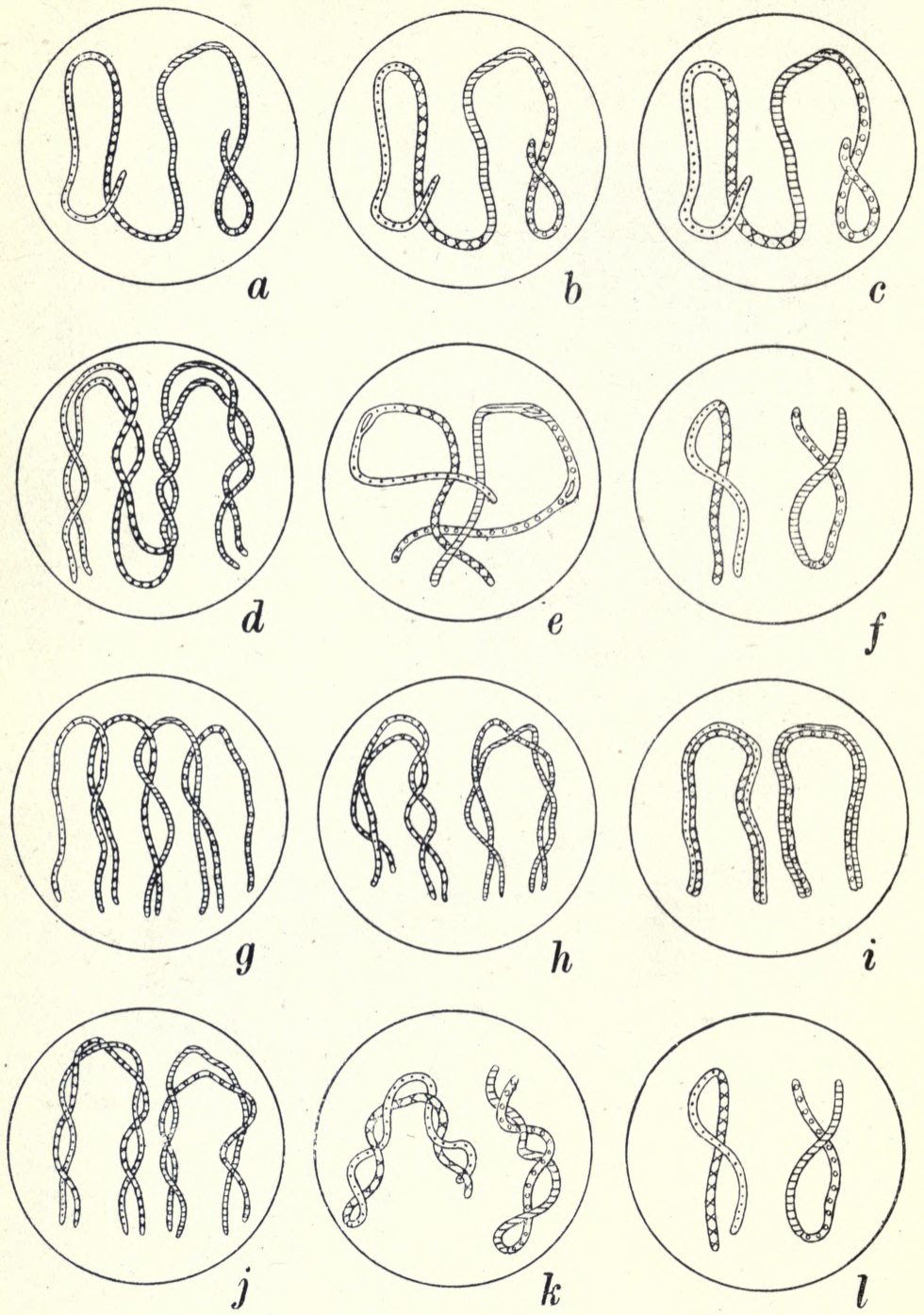

Diagrams illustrating the hypotheses of Telosyndesis $(a-f)$ and Parasyndesis $(g-l)$, slightly modified from GRÉGOIRE. 
however, the disagreement arises not from difference of material, but from divergent interpretations of the same phenomenon, and it will be necessary to devote a short space to the consideration of some of those points which have been subjects of controversy, in consequence of their theoretical importance.

One of the phenomena which has led to the greatest amount of discussion is that of the production of the double threads of the "bouquet" stage from the thin single threads of the early first spermatocyte nucleus. In Lepidosiren, according to Agar, the double threads are produced quite clearly by side-to-side union of the single threads seen at the rather earlier leptotene stage (Pl. VI, $h, i)$, and at the present time the opinion that this is the true account is held by an increasing number of cytologists (for example, A. and K. E. Schreiner, T. H. Montgomery, E. B. Wilson, A. Bolles Lee, etc.). Until recently, however, the generally accepted view was that the doubling of the threads in the zygotene stage was due, not to the parallel conjugation of the single leptotene threads, but to their longitudinal division, and this opinion is still held by a number of leading cytologists (P1. VI, $a-e$ ). They maintain that the leptotene threads thicken and then divide; the threads can often be seen to consist of a row of granules (microsomes) like a string of beads, and it is said that the granules increase in size in a direction transverse to the thread, and then each divides into two, thus giving rise to a longitudinal split along the whole thread. Some of those, on the other hand, who believe in the parallel conjugation of the threads, maintain that this is brought about by conjugation of the granules, each granule of one thread uniting with the corresponding granule of its neighbour. Whichever view is adopted in regard to the origin of the double threads 
of zygonema, it is generally agreed that the threads shorten and thicken, and that the split dividing the two halves disappears, in the succeeding pachytene stage. But there are again two entirely different interpretations of the strepsitene loops which next make their appearance. Some observers, whether they believe in the parallel conjugation of threads or in the longitudinal splitting, agree with the interpretation adopted by AGAR that the loops in strepsinema arise by the separation of the longitudinal halves of the double zygotene threads; they say that the longitudinal split between the two halves, which temporarily disappeared in pachynema, reappears and widens, so that the two halves of the double thread come apart along their whole length except at their ends, thus producing elongated loops. Another school, however, maintains that the strepsitene threads are still double, although the split which disappeared in pachynema is still invisible, and they regard the loops as consisting of two threads united end to end, and each of them potentially split longitudinally along its whole length. The two views thus seem at first sight to differ in a point of considerable importance - on the question whether the reduced number of double chromosomes which emerges from the synizesis clumping consists of pairs united end-to-end or side-to-side. Such descriptions as that of AGAR in the spermatogenesis of Lepidosiren, however, provide some sort of reconciliation between the two conflicting opinions, for they interpret the phenomena as indicating first a side-to-side conjugation (parasyndesis), followed by a separation of the united threads everywhere except at their ends, with the result that in strepsinema the rings each consist of two chromosomes united to each other at both ends. Although, therefore, workers of this school accept the view that there is side-to-side pairing in zygonema, they do not admit, as 
some have maintained, that the strepsitene rings consist of double chromosomes bent round on themselves like a split key-ring, a view which is not easy to reconcile with the later stages but which seemed at one time a natural corollary to the side-to-side pairing hypothesis.

-After the formation of the ring- or loop-shaped double chromosomes which undoubtedly emerge from the synizesis or contraction phase, whatever view is taken with regard to their origin, there is certainly a real difference in their behaviour in the next stage ("diakinesis") in different animals. In Lepidosiren, as has been seen above, the chromosomes emerge in the reduced number (half the somatic number) of double chromosomes, and then break up into their component parts, giving rise to the somatic number of single chromosomes. Almost immediately they then pair for the second time, giving the reduced number of doubles (sometimes called gemini or bivalents), arrange themselves on the spindle, and separate in such a way that the two members of each pair go to opposite poles of the spindle. This separation and reunion of the members of the pairs is, however, probably exceptional; more frequently the double chromosomes (gemini) which emerge from synizesis contract to form compact ring-, loop- or $\mathbf{X}$-shaped chromosomes which then arrange themselves directly on the spindle and separate into their component halves in the heterotype division. When, as in Lepidosiren, a separation and fresh pairing takes place, it is comparatively easy to see that complete single chromosomes segregate from each other in the heterotype division, but when no such second pairing takes place it is less easy to be certain of this. It has been maintained that in some animals the first spermatocyte division does not separate complete chromosomes, but that it consists in a longitudinal splitting of double chromosomes 
composed of two members united end-to-end, and that the separation of the members of the pair does not take place until the second division. In rare cases this may possibly be true, but it is now generally agreed that the first spermatocyte mitosis is normally the "reducing" division, that is, the division at which complete chromosomes segregate from each other, and that in the second division the chromosomes split longitudinally as in an ordinary somatic mitosis. The split which takes effect in the second division is often visible in the anaphase of the first or, according to some observers, even considerably earlier.

One further point about the origin of the gemini requires notice. In a number of Arthropods and Worms the emergence of the somatic number of chromosomes, each longitudinally split, has been described as following synizesis, and it is said that these then pair end-to-end, contract, and give rise to gemini which, in consequence of the split in each member, appear four-fold, and have hence been called tetrads. In other cases, especially in Copepods, the reduced number of longitudinally split chromosomes appears after synizesis; each has a transverse break in the middle, and they have therefore been regarded as being pairs of longitudinally split single chromosomes united end-to-end, the break corresponding with the point of union (Pl. VII). As in the preceding case, they contract and form tetrads. In the first spermatocyte division the tetrads each divide into two "dyads" (interpreted as single split chromosomes) and in the second division this split takes effect and divides the chromosomes into their component halves. Until some fifteen years ago this method of reduction by means of tetrads was regarded as almost universal, but more recent work has thrown grave doubt on the correctness of the interpretation. As has been seen above, the gemini formed by the second pairing in 


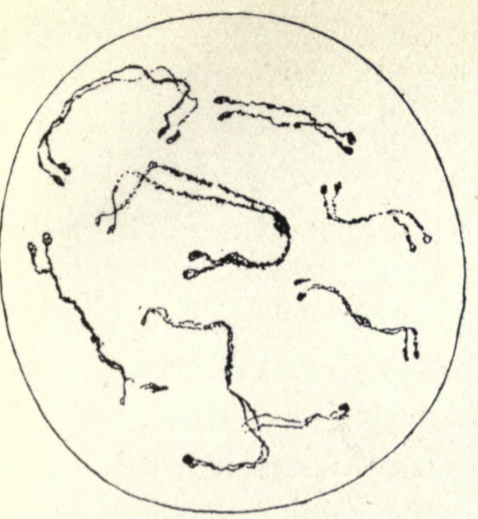

a

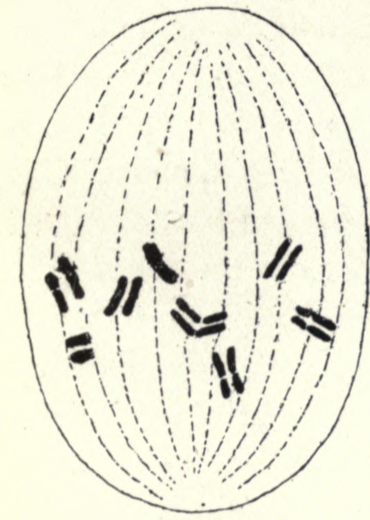

c

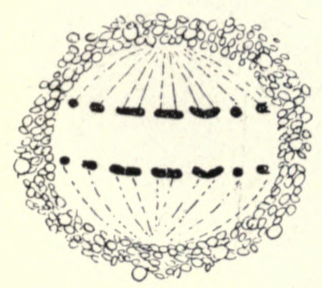

$e$
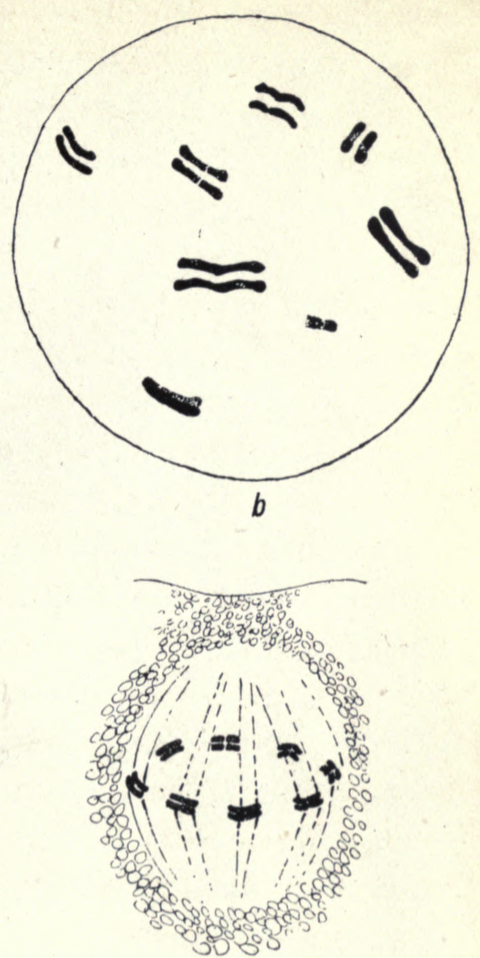

d

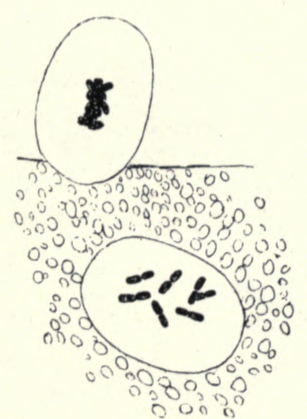

$f$

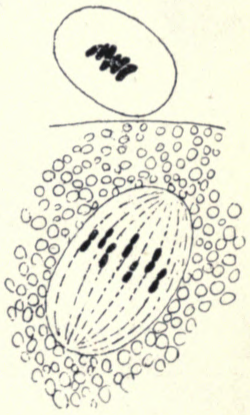

g

Semi-diagrammatic figures of maturation of egg of Cyclops, from RücKERT.

a. Early prophase of nucleus, long double threads. $b$. Late prophase, threads shorten and thicken. $c$. First appearance of spindle: the chromosomes have become tetrads by a transverse break. $d$. Metaphase, first polar mitosis. e. Anaphase. $f$. Prophase of second polar mitosis - the chromosomes appear as dyads. $g$. Second polar spindle-dyads dividing into univalents. 

Lepidosiren have a four-fold appearance, closely resembling typical tetrads, due to the constriction of each single chromosome in the middle, but this constriction in no way corresponds with a subsequent plane of division. Similarly the transverse break which has been assumed to be the point of attachment of the single ("univalent") chromosomes to each other at the first appearance of the gemini in Copepods is quite possibly a constriction of the same kind, and these bivalents may thus with great probability be regarded as consisting of pairs united side-to-side, rather than endto-end.

Whatever view is taken of the origin of the gemini or bivalents, it is clear that the final result consists in the separation of single chromosomes (univalents) from one another in one division, followed (or possibly in rare cases preceded) by a longitudinal splitting of these single chromosomes in the other. Since this result is the same whether the bivalents are formed by side-to-side or end-to-end pairing, it may seem unnecessary to devote so much space to the discussion of that part of the process, but, as will be seen in a later chapter, very important theoretical speculations have been based on the assumption that side-to-side pairing normally takes place, and these speculations would be meaningless if end-to-end pairing were in fact the rule.

Some objections that have been brought against the generally accepted hypothesis that the "reduction division" separates complete chromosomes from each other will be considered in a subsequent chapter. 


\section{CHAPTER VI}

The Maturation of the Germ-cells (continued):

The Maturation of the Egg and the Nature of the Meiotic Phase

$T^{N}$ the account of the maturation of the germ-cells given hitherto attention has been confined to the phenomena as seen in the development of the spermatozoon, since in the majority of animals they are more easily followed in

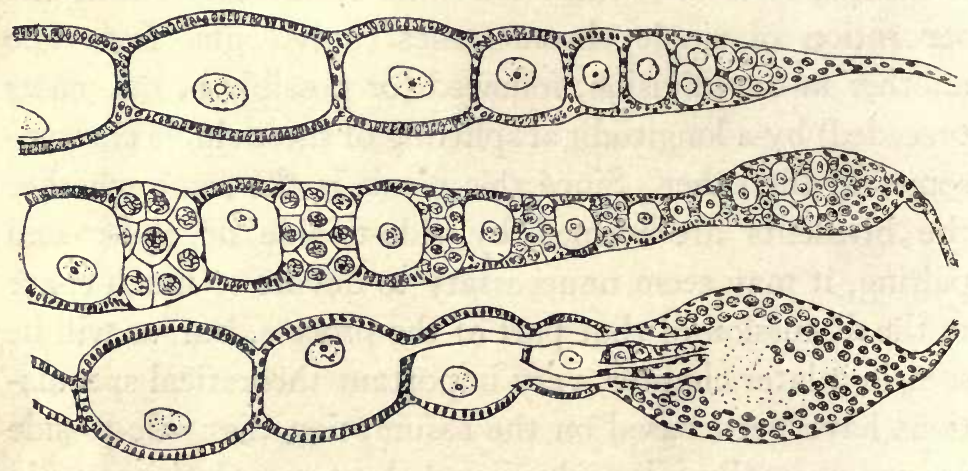

FIG. 8. Three types of Insect ovarian tubes.

- (After Korschelt and Heider.)

Above. Orthopteran type, without nutritive chambers.

Middle. Coleopteran or Hymenopteran type, nutritive chambers alternating with ova.

Below. Hemipteran type, single large nutritive chamber connected with ova by ducts.

the male than in the female. In the development of the egg, however, the process is exactly analogous, differing only in those features which depend on the inevitable difference between the production of the minute, active spermatozoon and the relatively large, inert, and often yolk-laden egg. 
Like the spermatozoa, the eggs are developed from small, comparatively undifferentiated cells known as oogonia, but not all the oogonia give rise to eggs, for many, in the majority of animals at least, are used up in forming accessory cells of various sorts. The process is especially well seen in the ovaries of Insects, in which the ovary consists of "tubes" or strands consisting of oogonia at the apex, while lower down the tube all the stages of the growth of the eggs and the differentiation of the accessory oogonia into follicle or nutritive cells can be seen in regular order.

In such an ovary the oogonia may be seen in division near the apex; the mitosis is of the ordinary somatic type and the chromosomes have the somatic number. After the last oogonial division the cells begin to enlarge and become oocytes, and the nucleus goes through precisely similar stages to those seen in the spermatocytes of the same species. The nucleus of the young oocyte, like that of the spermatocyte, consists of a tangled network of threads; from this a spireme is formed in which the thread appears for a time double, probably from the pairing of the reticular threads side by side, but according to some by their thickening and splitting. The spireme goes through the "bouquet stage," followed by synizesis, and from this emerge the reduced number of strepsitene loops, just as in spermatogenesis. The bivalent loops then contract to some extent, giving rise to half the somatic number of fairly short, thick threads, but do not, at this stage, proceed to form the typical gemini of the heterotype division. In the insect ovary (at least in many forms) an interesting differentiation takes place at this stage. In the cells which will develop into eggs the threads remain comparatively long, the nucleus continues to enlarge, and the deposition of yolk in the cytoplasm commences. In other cells, which up to this 
stage have appeared identical, the bivalent chromosomes contract into typical heterotype gemini and the univalent members of each pair then break apart and so give rise to the somatic number of single chromosomes. These latter cells then develop into accessory (nutritive) cells, and never form eggs.

The true oocytes, after reaching the condition in which the nucleus contains the reduced number of thick threads, may undergo a very long period of growth and waiting before the actual mitosis, of which the events described are the preliminary stages, takes place. In an egg which when mature contains much yolk the whole of it must be deposited in the interval, and while this is happening remarkable changes may occur in the nucleus, intercalated, so to speak, between the preliminary and final stages of the division. Very commonly the chromosomes, which up to this stage have been clearly defined and deeply staining, disappear, and the chromatin becomes scattered through the nucleus in the form of fine particles, or for a time it may vanish altogether, at least in the sense that it ceases to take up stain (cf. Pl. VIII). These changes in the nucleus between the first formation of the bivalent chromosomes and the division which separates them into their component halves are doubtless due to the great changes that are meanwhile taking place in the egg. Up to the time of the emergence of the double chromosomes from synizesis the oocyte has been quite small, little larger than the corresponding spermatocyte, but it now begins to increase greatly in size, and, in many animals, to accumulate a large amount of yolk, so that its volume may enlarge to many thousand times that of the oogonium from which it has developed. The nucleus, as has been seen in an earlier chapter, in some way controls the metabolic activities of the cell, and its 

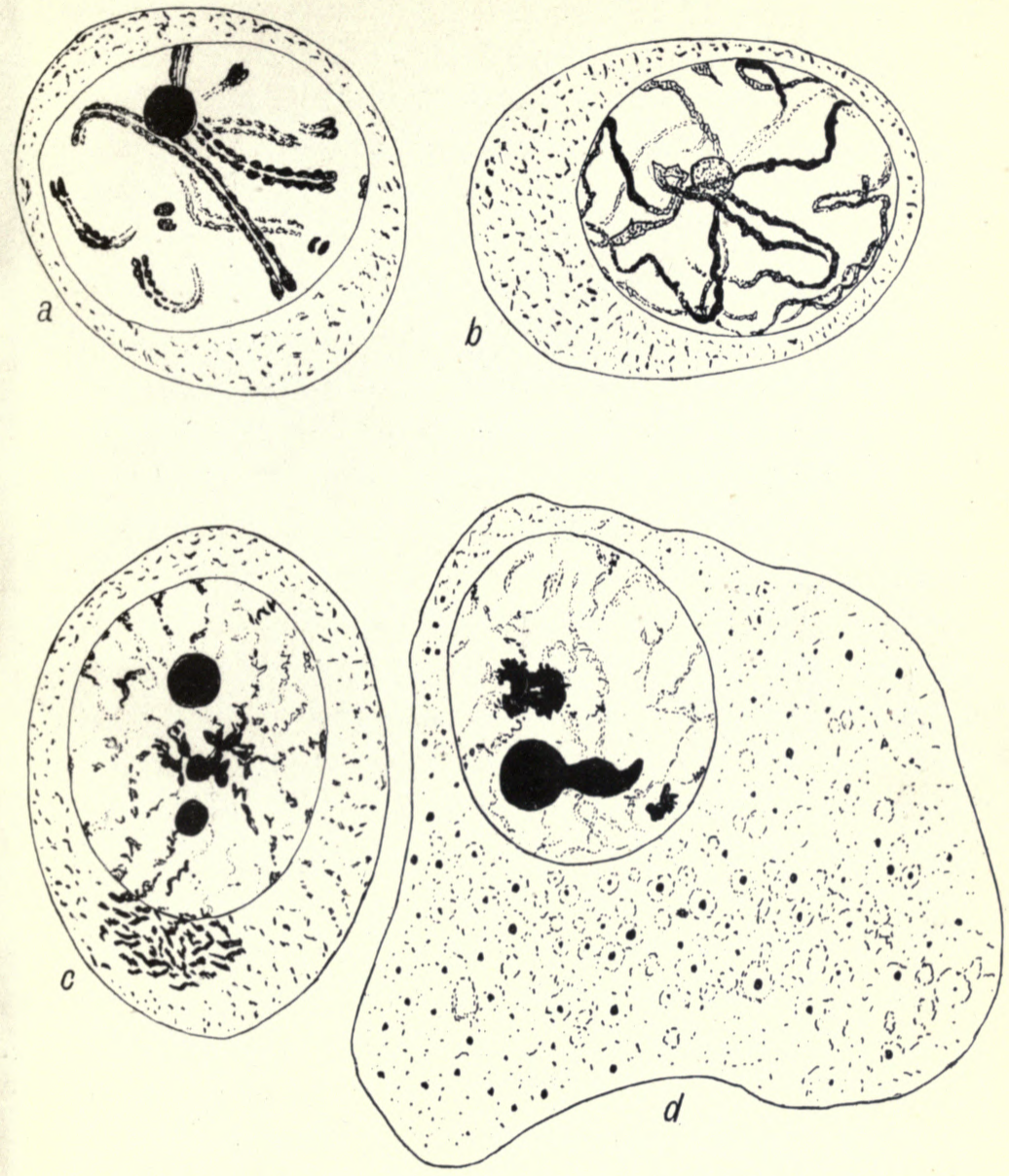

Stages in the development of the oocyte of Paludina (after Popoff, 1907).

a. Zygotene stage of chromatin threads.

b. Chromatin threads beginning to break up.

c. The nucleus has reverted to a network with scattered chromatin. Production of "chromidia" in preparation for deposition of yolk in cytoplasm*.

d. Cytoplasmic growth and deposition of yolk.

* The "chromidia" stain with osmic acid like Golgi apparatus, and are probably of the same nature (PopofF). 

peculiar behaviour in the growing oocyte can only be ascribed to its activities in this connection. In some eggs it may be seen that the first deposit of yolk takes place in the immediate neighbourhood of the nucleus. It must probably be supposed that in the early stages of gametogenesis, which correspond in the two sexes, the chromosomes are prepared for the "reducing" division which is to follow, and to which the spermatocytes proceed directly, while in the egg the framework, so to speak, persists, although the chromatin particles are then distributed through the nucleus in accordance with their function of controlling the growth of the egg-cell and the deposition of yolk. Sometimes staining granules appear at this stage in the neighbourhood of the nucleus (cf. Pl. VIII, c), which have been interpreted by some observers as chromatin discharged from the nucleus into the cytoplasm in connection with the deposition of yolk. Other investigators, however, maintain that these granules are not chromatin, but are true cytoplasmic structures, probably either mitochondrial in nature or belonging to the Golgi apparatus to which reference has been made in an earlier chapter. Popoff (1907) in the paper from which Pl. VIII is taken, points out that the granules, which superficially resemble chromatin, stain in the manner characteristic of the Golgi apparatus.

In some animals, such as Mammals, although very little yolk is deposited in the egg, there may be an extremely long waiting period between the preliminary stages of the maturation of the oocyte and the nuclear divisions which complete them. In Mammals the oocytes develop as far as the strepsitene stage before or shortly after birth, and the actual maturation divisions do not occur till the egg is ready for discharge from the ovary, so that in the longer- 
lived species as much as fifty years may elapse between the prophase of the division and its completion.

While the growth of the oocyte and the deposition of yolk are taking place the nucleus is very large, appearing as a conspicuous vesicle, usually with a large nucleolus (plasmosome). In the older works on embryology the nucleus at this stage was called the "germinal vesicle," and the nucleolus the "germinal spot." When the egg becomes full-sized, and approaches the final stages of maturation, the nucleus shrinks to a very much smaller volume, sometimes becoming so small as to be found only with difficulty. The chromosomes then reappear, often tightly packed together, and the egg is ready for the "maturation-divisions," which prepare its nucleus for conjugation with the spermatozoon.

In spermatogenesis, as has been seen in the preceding chapter, each primary spermatocyte divides twice, giving rise to four spermatozoa. In the egg also two nuclear divisions occur in rapid succession, but instead of the egg-cell dividing into two and then four equal cells, the division is extremely unequal, and the minute cells which are separated off from it are called polar bodies (Pl. IX). The first maturation or polar mitosis may take place in the ovary or after the egg is discharged; in some animals the act of laying seems to be the stimulus, and in others the polar division only occurs when a spermatozoon enters the egg. Typically the oocyte nucleus comes to lie near the surface of the egg, usually enclosed in a little patch of protoplasm free from yolk. A spindle is then formed perpendicular to the egg-surface, with its outer pole at the extreme margin; the nuclear membrane disappears and the bivalent chromosomes arrange themselves in the equatorial plate. A heterotype division follows, the bivalent chromosomes divide into their component halves, and these travel to the poles of the spindle. 

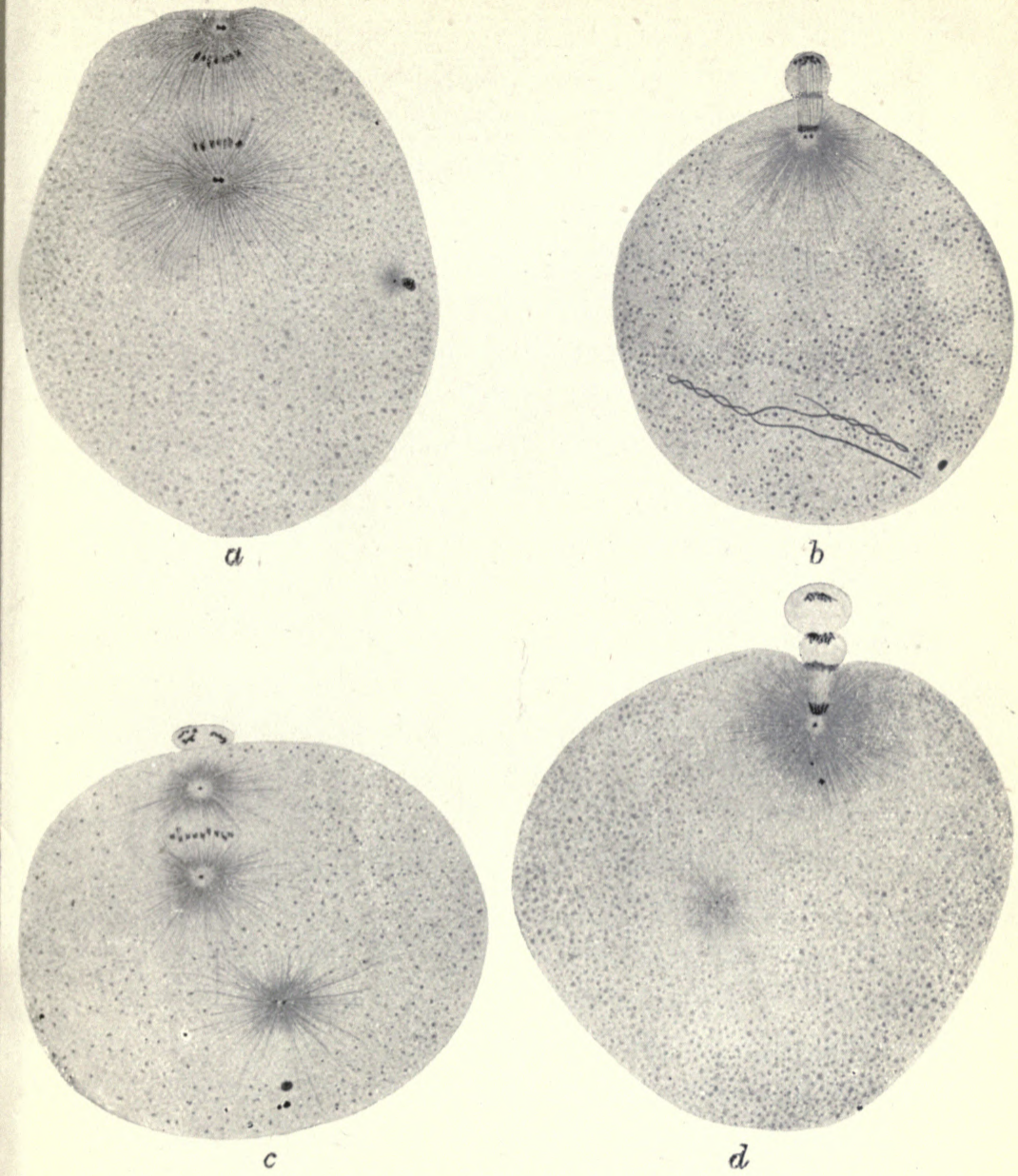

Four stages in the production of polar bodies in the egg of the Mollusc Pbysa fontinalis. From KosTANECKI and WiERzEJSKI.

a. Anaphase of first polar mitosis; on the right, sperm head with aster appearing in front of it.

b. Telophase of first polar mitosis ; polar body being extruded. Below, head and tail of spermatozoon.

c. Metaphase of second polar mitosis ; first polar body completely extruded. Below, sperm aster with divided centrosome widely separated from sperm head.

d. Telophase of second polar mitosis, and extrusion of second polar body.

All the figures are reconstructed from more than one section. 
Those which go to the outer pole at the egg-margin form a nucleus which is pushed out of the egg, possibly by the elongation of the spindle, together with a very small amount of cytoplasm; this constitutes the "first polar body." The other group goes to the inner pole of the spindle, and usually also forms a vesicular nucleus for a short time. In most animals the second division follows rapidly, though in some a considerable interval may elapse; a new spindle is formed, the egg-nucleus again is resolved into chromosomes which split and separate as in the homotype division of the secondary spermatocyte. Again the outer group of chromosomes travels to the edge of the egg and is extruded as a second nucleus enclosed in a small mass of cytoplasm, constituting the second polar body. Sometimes, but not in all animals, the first polar nucleus divides concurrently, so that the whole process gives rise in all to three minute polar cells and one large nucleated egg-cell. The nucleus of the egg-cell, sometimes now called the "female pronucleus," then sinks into the egg and is ready for-conjugation with the sperm-nucleus, which in many animals is already present in the egg at this stage.

The maturation phenomena in the egg are thus closely comparable with those of the spermatozoon. The early stages are very similar; then follows a period of growth and usually of nuclear change not represented in spermatogenesis, but when this is completed two successive nuclear divisions occur, giving rise in all to four cells as in spermatogenesis (cf. diagrams in Text-fig. 7, p. 6I). There is, however, the conspicuous difference, due to the great size and elaboration of the egg, that instead of these cells all being similar, one is a large, functional germ-cell, while the other three are minute bodies which in most cases are thrown out of the egg and lost. The differences, how- 
ever, are evidently superficial, depending on the nature and function of the cells concerned; fundamentally the process is similar in the two sexes. This similarity is further illustrated by the observation of FRANCOTTE that in the Polyclad Prostheceraeus the first polar body is nearly as large as the egg-cell. It undergoes an unequal division, giving off a small second polar body, just as the egg-cell does, and may be fertilised by a spermatozoon and segment as far as the gastrula stage. This production of a giant polar body has been imitated by Con KLIN (1917) in the eggs of the Mollusc Crepidula by centrifuging them during the maturation divisions. His results lead him to formulate an interesting hypothesis to account for the rather puzzling fact that in the production of normal polar bodies the cell divides extremely unequally in a way that contradicts the rule of protoplasmic mass referred to in Chapter III. He finds that if an egg is centrifuged violently during the prophase of the polar division, the whole polar spindle may be carried from the egg surface into the centre. If, however, the mitosis has reached the metaphase or anaphase stage, it is impossible to draw the outer pole of the spindle from the surface, with the result that the whole spindle becomes greatly elongated, and when the cell divides, the polar body separated off is relatively enormous. He concludes, therefore, that the polar spindle is firmly anchored, especially in the later stages, to the elastic "spongioplasm" of the egg surface by means of its outer pole or aster, while the inner pole is relatively free. The inequality of the polar divisions thus arises from the firm attachment of the mitotic spindle to the egg surface by one pole only, while in ordinary divisions, such as of a segmenting egg or of a spermatocyte, the poles are usually symmetrical in this respect. In this connection, and as showing still further the homology of 
the polar and spermatocyte divisions, it may be mentioned that very unequal spermatocyte divisions occur in the Bee, Aphids and Pediculus. In these cases one centrosome is drawn out at the tip of the finger-like process of the cell, and in the telophase of the mitosis this process is constricted off, much as a polar body is separated from an egg-cell.

In insects and some other animals the polar divisions are of a somewhat different type from that described above, though retaining the same essential features. The difference
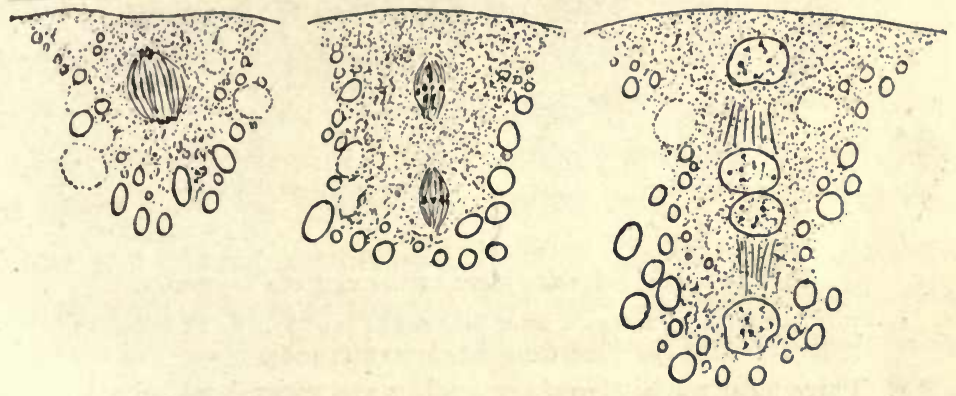

Fig. 9. Stages in the maturation of the egg of the Sawfly Nematus.

First polar mitosis, second polar mitosis, and close of second mitosis with egg-nucleus below and three polar nuclei, of which the two inner unite. (From the writer's preparations.)

consists in the fact that the polar nuclei are not extruded from the egg, but remain within it and usually degenerate in a short time. The first maturation division begins as described above, but the outer pole of the spindle is not quite at the egg margin, and the mitosis gives rise to two groups of chromosomes, one close to the surface and one rather deeper. Each group may form itself into a nucleus, or in some insects the second division follows so rapidly that this does not occur, and two new spindles are formed immediately, lying end to end perpendicular to the egg surface, with the two groups of chromosomes from the first division

\section{nic.}


in their equatorial plates. The two nuclei or chromosome groups then divide again, giving rise to four groups of chromosomes, which usually all form themselves into vesicular nuclei. Of these the two outer, at the poles of the outer spindle, are the halves of the first polar nucleus, and the other two are the second polar nucleus and the mature egg-nucleus. In some insects the polar chromosomes never

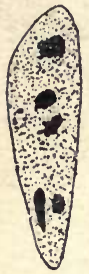

1

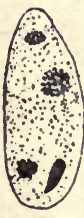

2

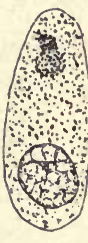

3

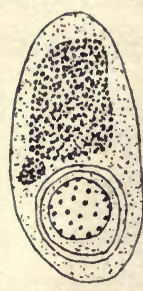

4
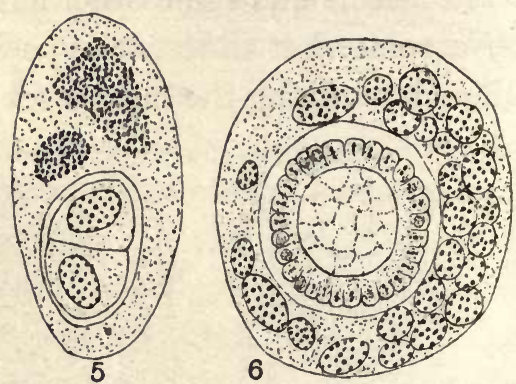

FIG. I0. Early development of the egg of Platygaster.

I-5. Platygaster dryomyiae, after Silvestri (1916). 6. P. ornatus slightly modified from MARCHAL (i 906 ).

1. Three polar nuclei above, egg-nucleus and sperm-head below.

2. The two inner polar nuclei have fused.

3. The egg and sperm nuclei have fused.

4. The protoplasm surrounding the zygote nucleus is separated as a distinct cell ; the fused polar nuclei are giving rise to the "paranucleus" of the trophamnion.

5. First segmentation division.

6. Blastoderm stage; paranucleus giving rise to nuclei of trophamnion. See also Fig. 20, p. 181 .

form vesicular nuclei, but remain as groups of chromosomes which gradually disintegrate. Not infrequently the second polar nucleus conjugates with the inner half of the first, or in some cases all three polar nuclei unite. With very rare exceptions, however, the polar nuclei, whether united or separate, disintegrate and disappear; the only known exception is in certain parasitic Hymenoptera (Chalcididae, Proctotrypidae), especially in those in which polyembryony 
(embryonic fission) occurs. In some of these it has been shown by SilvesTRI that the united polar nuclei persist and later divide and give origin to the nuclei of the envelope ("trophamnion") which encloses the mass of embryos into which the egg divides. In most species of the same families in which there is no embryonic fission, the polar nuclei degenerate, but Silvestri finds that Platygaster is an exception to this rule in having a trophamnion with nuclei derived from the polar nuclei (Text-fig. 10).

In all eggs, therefore, which are destined to be fertilised the maturation phenomena are closely comparable with those found in developing spermatozoa, and a similar process is found in the development of the spores of the Vascular Cryptogams and of the pollen-grains and embryo sac of the Flowering Plants. In all these cases the essential feature is the formation of double or bivalent chromosomes which appear in half the somatic number, followed by two rapidly succeeding divisions, in one of which the bivalents separate into their constituent univalent halves, and in the other the univalents divide by a longitudinal split. Each mother cell thus gives rise to four daughter cells containing half the somatic number of chromosomes; in the male all these cells develop into gametes, while in the female three are small and degenerate and only one mature gamete is formed.

It remains to consider some points in connection with the theoretical interpretation of the maturation-process or meiosis. Of these perhaps the most important is the constitution of the bivalents. The somatic number of chromosomes, sometimes called the diploid or " $2 n$ " number, is produced in the fertilised egg by the union of the egg and sperm. nuclei each of which contains the reduced (baploid or " $n$ ") number, and, as has been said above, the object of the 
maturation process is to produce germ-cells which again have the reduced number. But it is obviously of great importance to know whether the chromosomes unite indiscriminately in pairs to give rise to the bivalents, or whether the chromosomes differ among themselves and constitute a definite series which must be handed on unchanged to the next generation. If the chromosomes are all alike, it is evident that any one may pair with any other, but if they differ among themselves, one of each kind must obviously go into each daughter cell if the two cells are to have similar complements. In most animals it is quite easy to see that the chromosomes differ among themselves in size and in the shape which they assume in the metaphase and anaphase of the division, and especially in insects it is often possible to recognise each member of the series of chromosomes by means of careful measurements. It is then found that in the somatic and spermatogonial or oogonial cells there are two of each kind, so that these cells contain a double set (whence the expression "diploid" or $2 n$ number), and it is clear that the two sets are descended from the chromosomes introduced into the zygote by the two germcells which conjugated in its production. The diploid number may thus be said to consist of similar paternal and maternal haploid sets mixed together. When, as in Lepidosiren, it is possible to see the chromosomes coming together in pairs before the heterotype division, it is perfectly evident that similar or homologous chromosomes unite to form the bivalents, but even when the union takes place only in the spireme stage, and no separation and reunion of the members takes place in diakinesis, it is equally clear that each bivalent consists of two similar halves, for the univalent components are exactly alike as they separate in the anaphase of the heterotype mitosis. If, then, we call 
the set of chromosomes introduced into the zygote from the male parent $A, B, C, D, \ldots$, and the corresponding maternal set $A^{\prime}, B^{\prime}, C^{\prime}, D^{\prime}, \ldots$, the somatic or diploid series consists of $A, A^{\prime}, B, B^{\prime}, C, C^{\prime}, \ldots$, usually mingled indiscriminately together ${ }^{1}$. When, however, the meiotic phase in the maturation of the germ-cells supervenes, the chromosomes pair together, $A$ with $A^{\prime}, B$ with $B^{\prime}$, and so on, so that each bivalent in the heterotype division consists of two homologous chromosomes, one of which is of paternal and the other of maternal origin. Since the two of each pair are commonly alike it is not possible to say from direct observation whether all the paternal chromosomes go to one pole of the spindle, and all the maternal to the other, or whether they behave irregularly, but a comparison of the chromosome behaviour with the facts of heredity leads to the definite conclusion that in respect of any pair it is a matter of chance whether one or the other member goes to a given pole. This conclusion is confirmed by observations such as those of Miss CARothers (1917) on the rather rare cases in which the members of a pair may differ in size or in the position at which the spindle-fibre is attached. Miss Carothers found that in a grasshopper the spindlefibre may be attached at the end of the chromosome, giving a rod-shape in anaphase, or nearer the middle giving a $\mathbf{J}$ shape. In the spermatocytes of some individuals rods and $\mathrm{J}$-forms were paired together. She then found that there was no regularity in the arrangement of these pairs on the spindle, but that the segregation of the members of different pairs was quite independent of one another. It must there-

1 In the Diptera in all the cells of the body, whether somatic or germinal, which have the diploid number, homologous chromosomes constantly lie next to one another in the equatorial plate, $A$ being associated with $A^{\prime}, B$ with $B^{\prime}$, etc. (METz). This is not usual in other groups of animals. 
fore be concluded that the daughter-nuclei may contain any combination of paternal and maternal chromosomes, as long as one and one only of each kind is represented; that is to say, any combinations such as $A, B, C_{2} \ldots, A$, $B^{\prime}, C, \ldots, A^{\prime}, B, C^{\prime}, \ldots$ are possible, but every letter must be represented once and once only. In other words, it appears that the function of the reducing division is to ensure that each germ-cell shall contain one complete

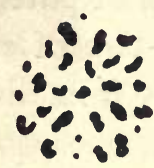

1

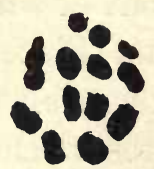

2

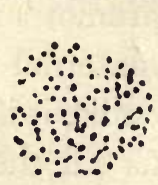

3

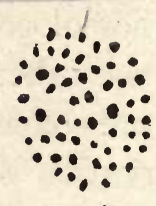

4

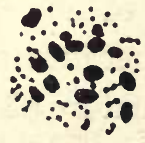

5

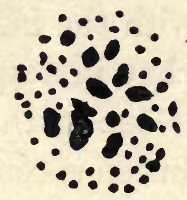

6

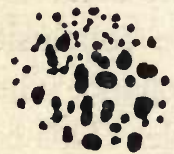

7

FIG. I1. Chromosomes of Biston hirtarius and Nyssia zonaria and the hybrid between them, from HARRISON and DONCASTER.

1, 2. B. birtarius, spermatogonial group (1) and first spermatocyte group (2).

3, 4. N.zonaria spermatogonial group (3) and first spermatocyte group (4). 5, 6, 7. Hybrid zonaria $q$ and hirtarius $\delta: 5$, spermatogonial group; 6,7 , first spermatocyte groups.

haploid set, but that within this set all possible combinations of chromosomes of paternal and maternal origin are possible.

That the bivalent chromosomes of the meiotic phase do in fact consist of pairs, one of which is of paternal and the other of maternal origin, is clearly shown by the behaviour of the chromosomes in certain hybrids, especially in Moths, and also in various plants. A particularly clear example is seen in the hybrid between the moths Biston birtarius and 
Nyssia zonaria ${ }^{1}$. In B. birtarius the reduced germ-cells contain 14 mostly rather large chromosomes of which two are usually united, giving the appearance of 13 ; those of $N$. zonaria have 56 small ones. The hybrid has 70 , some large and the rest small, in its spermatogonia. After the growthphase about 60 to 65 chromosomes emerge from the spireme, a few of which are evidently double (bivalent), while the majority are single (univalent), a few of these being large and the rest small. At the first division the bivalents divide into their components, and the rest divide equationally, and probably all divide equationally at the second division, producing spermatids with only a few less than the somatic number of chromosomes. The explanation is clearly that most of the chromosomes derived from the one parent are too unlike any of those derived from the other for pairing to be possible, so that hardly any pairing takes place, and the chromosomes in the maturation divisions are nearly as numerous as in the spermatogonial, instead of only half as many. A few of the chromosomes derived from the two parents, however, correspond sufficiently to be able to pair, and these give rise to the bivalents, which seem to vary in number from five to about ten.

Further evidence pointing in the same direction will be given in the chapter on the cytological phenomena of sex.

That the pairing and separation of the chromosomes in the meiotic phase brings about the required result is perfectly evident, but it is not so clear why the reducing division should always be followed by a second division of the equational type, that is to say, in which the individual chromosomes are split into longitudinal halves. A number of hypothetical explanations of this second division have been offered, but as most of them are quite devoid of

\section{Harrison and Doncaster, 1914.}


experimental support it is not necessary to consider them here. The most probable explanation is that the second maturation division represents the completion of a process which began at a much earlier stage, and that the heterotype mitosis is a peculiar form of division, intercalated between the prophase stages and the completion of an ordinary nuclear division. In an ordinary somatic division the chromosomes emerge from the nuclear reticulum and contract to form rods which are twice as thick as the chromosomes in anaphase, so that if the width of the chromosomes in anaphase is taken as the standard or unit, those in the prophase may be regarded as having twice the normal thickness. They then split longitudinally, and the two halves, now of the standard width, separate to the two poles of the spindle. In the meiotic phase the same process takes place, with the addition that the chromosomes of double width are now associated in pairs, so that each bivalent consists, actually or potentially, of two double rods lying side by side. The heterotype division separates the members of the pair, but since each member is of double width, and has often already a visible longitudinal split, the second (homotype) division is needed to complete the process and produce chromosomes of standard or unit thickness. This is illustrated diagrammatically in Textfig. I2. That this is the true explanation of the double division in the maturation of the gametes is strongly indicated by the phenomena found in most parthenogenetic eggs, and in the spermatogenesis of the Hymenoptera, which will be considered in a later chapter. In these cases, for reasons which will be explained, the reduction process is omitted; there is no pairing of chromosomes and the maturation consists of a single equational or homotype mitosis. 
Adifferent explanation of the occurrence of two successive maturations, due to JANSSENS, is of such great theoretical importance that it must be mentioned here, though it will be referred to again later. JANssEns (1909) maintains that
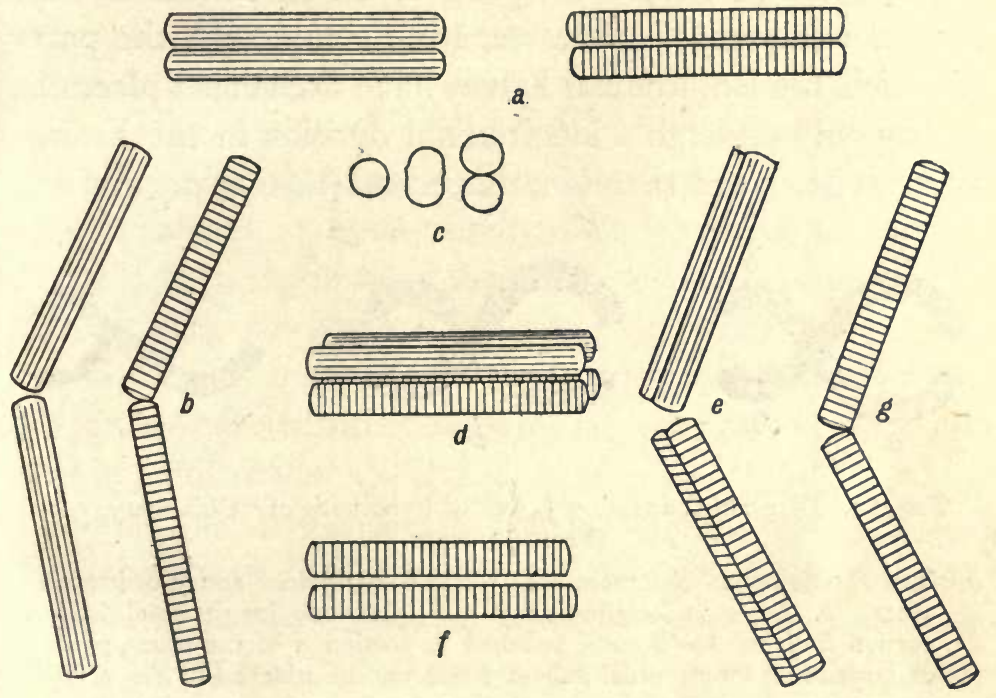

FIG. 12. Diagram of chromosome division in somatic and in maturation mitoses.

a. Two chromosomes in metaphase, and b. in anaphase, of a somatic or spermatogonial mitosis. c. Transverse section illustrating the doubling in thickness and splitting of a chromosome before division.

d. Bivalent chromosome of first maturation division, consisting of two univalents, each longitudinally split.

e. Anaphase of first maturation division, separating complete, split univalents from each other.

$f, g$. Metaphase and anaphase of second maturation division, separating longitudinal halves of the uniyalent.

when the chromosomes twist round one another, as they often do in strepsinema, they may fuse at the points of contact, and when they separate parts of the two homologous chromosomes may exchange places. He also gives evidence that when the heterotype chromosomes split 
longitudinally, part of one longitudinal half of one chromosome may exchange places with a similar part of the corresponding longitudinal half of the other. In consequence of this the heterotype division does not separate whole chromosomes, for while the parts of the chromosomes that have not exchanged places separate completely, the parts of which the longitudinal halves have exchanged places in reality only undergo a longitudinal division in the heterotype division, and therefore the second (homotype) division
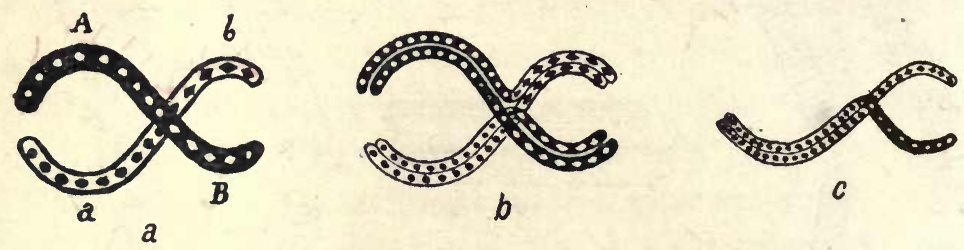

FIG. 13. Diagram illustrating Janssens' hypothesis of "Chiasmatypy."

(From Janssens.)

a. Two homologous chromosomes, $A B$ and $a b$, paired and crossing each other. $b$. Each is longitudinally split, and one longitudinal half of portion $B$ of one has become attached to portion $a$ of the other, while a corresponding longitudinal half of $b$ has become attached to $A$. c. Split chromosome in the anaphase of the first (heterotype) maturation division; one longitudinal half consists of portions $a b$, the other of $a B$.

The complete segregation of $A B$ from $a b$ into separate nuclei will only be effected in the succeeding homotype division which separates these longitudinal halves.

is needed in order to complete the separation. Reference to Text-fig. I 3 will make his scheme clearer. He points out that this scheme not only explains the curious crossshaped figures, which may show twisting at one end and not at the other, that are often seen in the heterotype division, but also accounts for the universal presence of two divisions in the maturation of the gametes, for both divisions are required in order completely to separate the homologous chromosomes into different nuclei. 


\section{CHAPTER VII}

\section{The Cytoplasmic structures in Spermatogenesis and Oogenesis}

\section{Spermatogenesis}

]$^{N}$ the foregoing account of the maturation phenomena of spermatozoa and ova attention has been directed almost exclusively to the nuclear changes; it will now be necessary to turn aside for a space, in order to consider some of the cytoplasmic processes characteristic of the development of the germ-cells, before describing the behaviour of these cells in fertilisation.

During the two cell-divisions which take place in the development of each primary spermatocyte into four spermatids, important changes commonly occur in the cytoplasm in preparation for the peculiar structure of the mature spermatozoon. The nature of these changes will perhaps be best understood if a short description is first given of a typical spermatozoon. With a few conspicuous exceptions, for example in the Nematode worms and the higher Crustacea, the spermatozoa throughout the animal kingdom are built on a common plan, although there are of course great differences in detail between those of members of different groups, or even of different species within one group. A typical spermatozoon consists of three parts, usually called head, middle-piece and tail. The head varies greatly in shape in different animals; it is perhaps most commonly oval in outline, but may have the form of an elongated rod, straight or sometimes bent, or it may be twisted into a cork-screw shape or have other peculiar 
forms. These spiral forms are an instance of the asymmetry which is frequent, if not general, in the structure of spermatozoa, and it has been suggested that the excentric position of the nucleus of the unfertilised egg is a manifestation of the same thing in the female gamete. The head consists chiefly of a highly concentrated nucleus, which stains intensely and almost evenly with chromatin stains. The nucleus is probably enclosed in a thin sheath of cytoplasm, although this is usually not readily visible. At its anterior end there is often a pointed apical body or acrosome, the function of which is probably to help in perforating the surface of the egg when the spermatozoon enters it. The

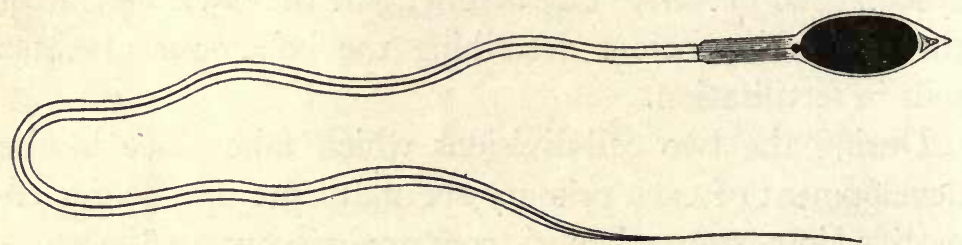

Frg. 14. Diagram of a spermatozoon, showing head with nucleus and acrosome, middle-piece with end-knob, and tail with axial filament. (To save space the tail is represented as bent on itself.)

middle-piece is usually short and cylindrical, and contains the base of the axial filament of the tail, which may be swollen at the front end of the middle-piece, at its junction with the head, into an "end-knob." It is this thickened base of the axial filament which becomes, or at least produces, the centrosome of the first segmentation division of the fertilised egg. The tail is a long tapering filament, actively motile like a flagellum, by the lashing of which the spermatozoon swims vigorously in the seminal fluid or in water. It consists of a thin outer sheath which stains only slightly, surrounding a fine axial filament arising from the base in the middle-piece and running throughout the length of the tail. 
The nucleus of the spermatozoon is formed directly from that of the spermatid, but usually goes through a characteristic series of changes. In many animals the nucleus of the young spermatid consists of a vesicle with the chromosomes all arranged immediately within the nuclear membrane. In these nuclei little or no reticular structure is visible, but the chromatin becomes spread out over the inner surface of the membrane so that in section the nucleus has the appearance of a darkly-staining ring, often at first with irregular lumps of chromatin projecting into the cavity. Gradually the nucleus contracts, and in animals which have spermatozoa with elongated heads it becomes (in optical section) at first oval and then a long narrow ellipse. Finally the clear inner space disappears and the nucleus in the head of the mature spermatozoon appears as a dense evenly stained mass of chromatin. Although the nucleus with an outer ring of chromatin and clear inner space is very characteristic of the spermatid, it is by no means universal, and animals of various groups have reticular spermatid nuclei with the chromatin scattered on the network, usually in rather coarse masses. The later development of these nuclei, however, differs very little from that of the type described above.

Apart from the nucleus, the only other structure of importance in the head of the spermatozoon is the acrosome, and it will be convenient to leave the discussion of its origin to a rather later stage.

While the spermatid nucleus is undergoing the changes just described, the whole cell is elongating, and important changes, which may have begun still earlier, are taking place in connection with the centrosome. During the spermatocyte divisions, which usually follow each other rapidly, the centrosomes (centrioles) have remained conspicuous objects, generally lying near the edge of the cell, and in the 
young spermatid, just after the last division, the centrosome is commonly just below the surface, and begins to grow out a filament which projects outside the cell-protoplasm. In some animals this outgrowth into a projecting filament may begin at an earlier stage; in Moths, for example, it occurs in the primary spermatocytes and the two centrosomes each develop two filaments, which separate from one another when the centrosomes divide for the second spermatocyte division (Pl. XI, 2, 3). More commonly the filament is only developed after the last cell division is completed; it grows out rapidly, and at the same time the centrosome itself sinks in to the neighbourhood of the nucleus, and sometimes pushes in the nuclear membrane so as to appear as if enclosed within the nucleus. At first the filament growing from the centrosome is naked, but as the spermatid becomes converted into the spermatozoon its cytoplasm grows out along the filament and gives rise to the cytoplasmic sheath or fin surrounding the axial filament of the tail. The tail thus arises from a filament which grows out from the spermatid centrosome and becomes clothed with a thin sheath of cytoplasm. The middle-piece at the same time originates chiefly from the centrosome itself and the thickened base of the axial filament, surrounded by part of the mitochondrial apparatus next to be described ( $\mathrm{Pl}$. X, $a-g$ ).

The formation of the middle-piece and tail is not, however, a simple growing out or lengthening of the cytoplasm of the spermatid around the axial filament produced from the centrosome, but is accompanied by important processes for the description of which it will be necessary to turn back to the earlier stages of spermatogenesis. In the young primary spermatocyte, when suitably stained, granules or strands may often be seen in the cytoplasm, especially in the neighbourhood of the centrosome. These bodies are the 
Plate $X$
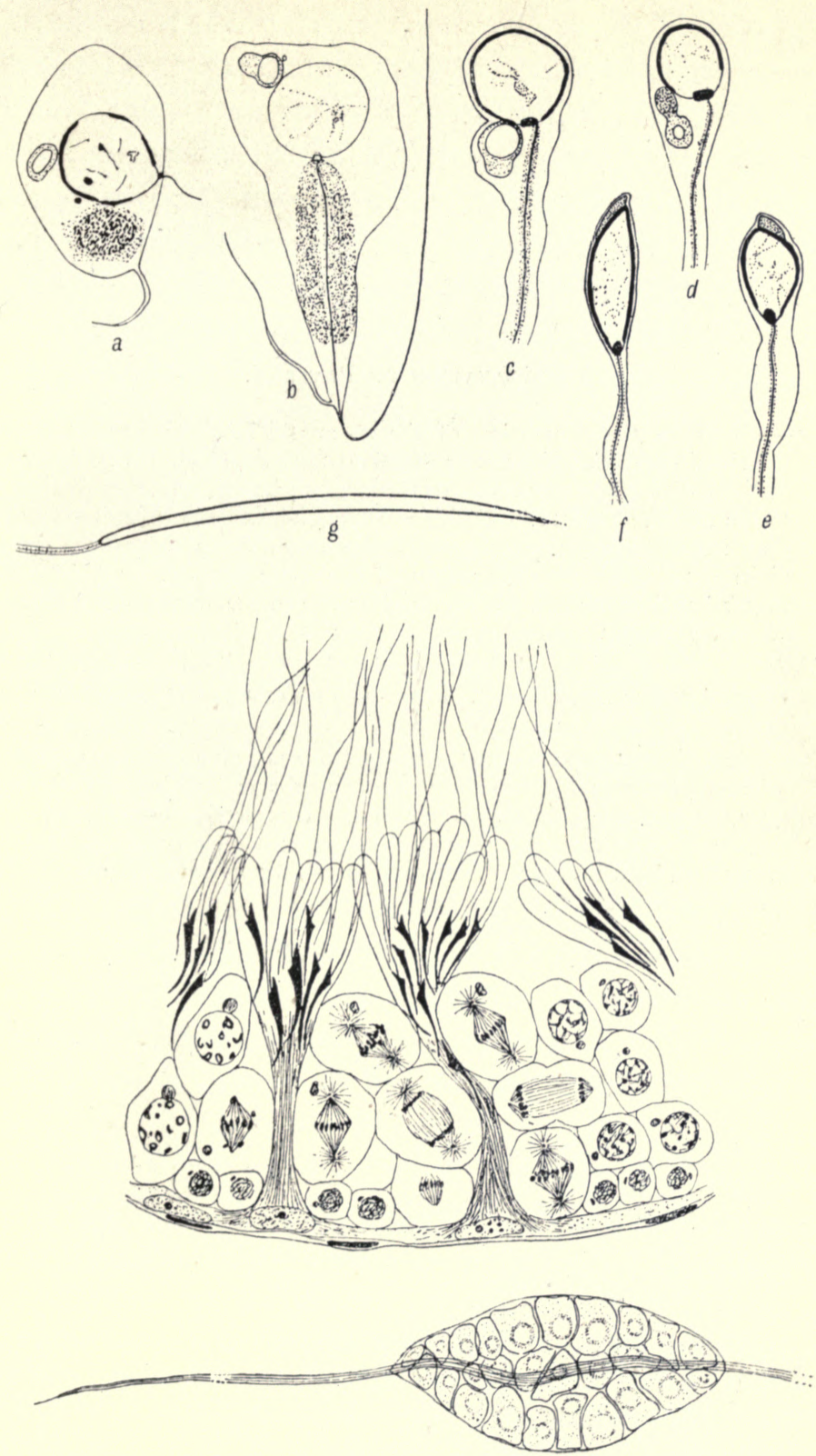


\section{Explanation of Plate $X$.}

Above-Stages of the conversion of the spermatid into the spermatozoon in the Hemipteran Euschistus, after Montgomery ( I 9 I I $a$ ). (a) In the centre, nucleus with centrosome and axial filament; below, mitochondrial body; to the left, the "sphere." (b) The mitochondrial body is beginning to be drawn out along the growing axial filament; the sphere shows signs of division. $(c),(d)$ Further stages of the same process. $(e),(f)$ The sphere has divided, and part of it gives rise to the acrosome. ( $g$ ) Head of nearly mature spermatozoon.

Middle - Part of testicular tubule of Rat, showing Sertoli cells to which bundles of spermatozoa are attached. From Korschelt and Heider, after LenHOSSEK.

Below - So-called apyrene spermatozoon of Vermetus, after KuschaKewiTsch. The cell-body is filled with "albumen-spheres" which are deposited after the chromatin of the spermatid has completely degenerated. The bundle of axial filaments (the full length of which is not represented) grows out from numerous centrosomes arising by multiplication of the spermatid centrosome. 
mitocbondria already shortly referred to in Chapter II. They were first observed in spermatocytes and spermatids, but have since been found in many other cells of various animals, especially in the embryo. To see them well the tissue must be preserved in a special manner, the most important point being that the fixative should contain no acetic acid. Theystain well, but somewhat capriciously, with Heidenhain's iron-haematoxylin, and particularly with BENDA's process. In the spermatocyte, as the cell enlarges, the mitochondrial bodies increase in size, and are usually described as becoming definite threads of staining substance which frequently take the form of loops or rings. In both the spermatocyte divisions they may appear to be drawn out parallel with the spindle, in a somewhat irregular manner, and as the cell divides they are separated about equally into the daughter cells. In the young spermatid they unite to form a fairly compact mass near the nucleus at the side of the cell at which the tail will grow out ${ }^{\mathbf{1}}$. In the stages for which GATENBY has proposed the name spermateleosis, that is, during the final development of the spermatozoon from the spermatid by the outgrowth of the cytoplasm along the axial filament (sometimes also called "spermiogenesis"), the mitochondrial mass becomes drawn out so as to form an elongated sheath enclosing the filament. Its final fate is uncertain, but will be shortly referred to below.

In testes fixed by the usual methods (for example Flemming's fluid, alcohol-sublimate acetic mixtures, etc.) designed especially for giving clear figures of the nucleus

1 This mitochondrial mass ("mitosome") constitutes the "Nebenkern" of some authors, but as the word has been used to designate the remains of the division-spindle ("Spindelrestkőrper," "spindle-bridge"), the "idiozome" (or "sphere") and other cell-structures, it is now dropping out of use. 
and chromosomes, the mitochondria have such appearance as is described and figured by Meves (1900, 1907), MontGOMERY (IgI I $a$ ), and others. More recently, however, GATENBY $(1917,1918)$ has re-investigated the subject in Moths and Molluscs and concludes that the appearances commonly seen are deceptive in consequence of faulty fixation. He finds that fixatives containing acid or alcohol either destroy the mitochondria or distort them so badly as to make accurate observation impossible. He maintains that the long strands frequently seen, especially in dividing cells, have in most cases no real existence, but are produced by the running together of bodies which are in life semifluid vesicles ${ }^{1}$. Some of his figures, taken from Moths, are reproduced in Pl. XI. According to Gatenby the mitochondria appear in Lepidoptera as a cloud of granules partially encircling the nucleus of the spermatogonium and early spermatocyte. During the growth-phase the granules become hollow and filled with a non-staining substance; the vesicles so formed enlarge and show a tendency to coalesce, but the coalescence at this stage may be due to the action of the fixative. During the spermatocyte divisions the mitochondrial vesicles become separated, apparently passively, into the daughter cells; they may be drawn out along the length of the spindle and coalesce to elongated strands, which then break apart, but there is no regular division of the individual vesicles, nor is it clear that their separation into the daughter cells is at all closely controlled by the spindle mechanism. In the spermatid the mitochondria take on a new phase. The vesicles begin to flow together and form a tangled filament, derived from the outer staining layer of the vesicles, enclosing in its meshes a non-staining substance

1 Paludina is an exception; its spermatocytes have bent, rod-shaped mitochondria. 


\section{Explanation of Plate XI.}

Mitochondria in the spermatogenesis of Lepidoptera, from Gatenby. (Reproduced by permission from Quart. Journ. Micr. Sci. LxiI. I 9 I 7, Plates 2 3, 25.)

I-5. Smerinthus populi; 6, Euchelia jacobaeae.

I. Spermatogonium with mitochondrial granules $(M D)$, around nuclear membrane.

2. Primary spermatocyte with mitochondrial vesicles $(M D)$, acroblasts $(A B)$, and two centrosomes $(C)$, with double axial filaments.

3. First spermatocyte division.

4. Spermatid showing mitochondrial vesicles coalescing to form the mitosome.

5. More advanced spermatid. The mitosome has assumed the form of a spiral thread around the axial filament $(F L)$. The acroblasts are applied to the nucleus as vesicles.

6. Head of a later spermatid, well advanced towards a mature spermatozoon. The acroblast vesicles have coalesced to form the acrosome $(A G)$.

\section{Explanation of lettering.}

$A B$, acroblast ; $A G$, acrosome ; $A X F$, axial filament ; $C^{1}$, proximal centrosome; $C^{2}$, distal centrosome of spermatid, which passes down into the tail; $C O$, non-staining portion of mitosome ; $F L$, axial filament ; $M$, micromitosome ; $M D$, mitochondria; $N$, nucleus; $S P$, mitochondrial spireme; $X Y$, outline of neighbouring cells. 
derived from their centres. The filament then arranges itself in a coil or spiral, and gives rise to the "mitochondrial spireme" ("macromitosome"), a conspicuous body lying immediately behind the nucleus of the now elongating spermatid. As the spermatid lengthens to form the spermatozoon, the macromitosome is drawn out and wrapped round the axial filament, but it becomes so attenuated that it could not be determined whether it remained as a final constituent of the tail.

In a number of Molluscs examined Gatenby finds the behaviour of the mitochondria in spermatogenesis to differ only in detail from that observed in Lepidoptera, with the exception that no mitochondrial spireme or macromitosome is formed, and the mitochondria pass as small vesicles in to the tail of the spermatozoon. He describes, however, two other kinds of cytoplasmic bodies to which reference must be made. The first of these he names micro-mitochondria; they differ from the larger "macro-mitochondria" not only in their smaller size but also in always forming a sheath round the proximal end of the axial filament. Since in some species there is no difference in size, although the mitochondria become separated into proximal and distal groups, these bodies probably differ little from true mitochondria. In some forms (for example the slug Arion) "post-nuclear gramules" are also found. These granules differ from mitochondria in staining like chromatin with certain aniline dyes (toluidin blue); they appear in the spermatocytes in the neighbourhood of the nucleus, and form a dense ring just behind it in the spermatid. A third body, often known as the "Nebenkern," which is conspicuous in Molluscs, will be referred to below.

Some writers have ascribed great importance to the mitochondrial bodies as a whole, and have supposed especially

D.c. 
that they play a predominant part in the transmission of inherited characters. For this there is little evidence, and it seems more probable that their main function is nutritional, and concerned in spermatogenesis with the rapid transformation of the spermatid into the spermatozoon. They are usually most conspicuous in cells which are about to undergo differentiation, and some workers have regarded them as purely temporary structures, produced by the physiological condition of the cell, perhaps as reserves of nutritive material. It seems to be established, however, that they have the power of division and propagation like the plastids of plant cells, and with our present very imperfect knowledge it seems best to regard them as living structures, of the nature of cell-organs, which may multiply and become conspicuous at times when rapid intracellular differentiation is about to take place. That they are intimately associated with cell-metabolism may be inferred from Montgomery's observation (I9II $a$ ) on the Hemipteran Euschistus, that in the spermatogonial nucleus part of the chromatin is always in contact with the membrane in one area, forming a "chromatin-plate," and that although the position of this plate varies, with respect to the rest of the cell, in different cells, the mitochondria and "idiozome" (dense protoplasm equivalent to GATENBY's archoplasm) always appear in the region of the chromatin-plate. This suggests that the mitochondria arise or at least multiply in connection with a process of nucleo-cytoplasmic reaction. Montgomery (1912) also maintains that in Peripatus the whole mitochondrial body is thrown out of the spermatid before its transformation into the spermatozoon is quite complete, a fact impossible to harmonise with the belief that mitochondria transmit hereditary characters.

The fate of the mitochondrial body of the spermatozoon, 
like that of the Golgi apparatus to be mentioned below, varies in different cases. In Ascaris, which has a spermatozoon with a considerable cell body, the mitochondria are carried over into the egg and there multiply and doubtless provide part of the mitochondrial apparatus of the embryo. In Echinoids, as Meves has shown, the mitochondrial body is carried into the egg as the "middle-piece," which is recognisable to a late stage of segmentation by appropriate staining as a distinct granule, but takes no apparent part in development; and lastly, if Montgomery is correct, the mature spermatozoon of Peripatus is without mitochondria. All this points to their being functional in spermatogenesis rather than as being bodies transmitted by the spermatozoon for the needs of the embryo.

The last element in the spermatozoon of which the origin must be described is the acrosome, about which there has been great divergence of opinion. According to Montgomery it arises from a body which appears in the spermatid after the second spermatocyte division, and has been called by various writers the sphere or idiozome. The word sphere, however, has often been used as equivalent to centrosphere, that is, the outer layer of "archoplasm" (Bove RI) enclosing the centrosome, and although the "sphere" of the spermatid is described in some cases as developing in close relation with the centrosome, in others there is no such connection, so that the two structures are probably quite distinct. The word "idiozome" introduced by Meves is therefore more satisfactory, but it is by no means certain that the idiozome of the spermatid is in any way equivalent to the body which Montgomery calls by that name in the spermatogonia (see p. 98). The idiozome or sphere, then, arises in the spermatid usually, but not necessarily, near the centrosome, and therefore usually in a position which would be 
behind the nucleus in the mature spermatozoon. As the tail grows out and the spermatid elongates, the idiozome gradually travels forward, so that by the time when the nucleus is being converted into the head of the spermatozoon the idiozome is in front of it and in close contact with its anterior pole, where it becomes concentrated and pointed in shape and gives rise to the apical body or acrosome. Usually the whole idiozome is described as being converted into the acrosome, but in Euschistus Montgomery finds that it divides into two parts, one of which travels forward to form the acrosome while the other remains behind the nucleus and seems eventually to disappear (Pl. X, $a-f)$.

In Lepidoptera, however, GATE nBy (I9I7 $a$ ) has given an entirely different account of the origin of the acrosome. In the primary spermatocytes he finds deeply stained rod-like bodies which from their subsequent fate he names acroblasts; they are usually curved with their concave sides lying toward the nucleus, and lying in parts of the cytoplasm free of mitochondria. Their number varies somewhat in different species. In the spermatocyte divisions they become distributed to the daughter cells, and their movements appear to be more definitely controlled by the astral rays than those of the mitochondria. In the spermatid they become first hemispherical, with a non-staining centre, and then round hollow vesicles. The vesicles, which in Smerinthus populi are from three to five in number in the spermatid, arrange themselves around the nucleus and then become adherent to it. At the point at which each vesicle touches the nucleus a staining granule is formed, and as the spermatid nucleus contracts to form the head of the spermatozoon the acroblast vesicles coalesce, and the contained granules unite to form a single body which gives rise to the acrosome (P1. XI, 3-6 $A B, A G$ ). 
GatenBy's later papers (1919 $b, c)$ bring these and other observations on the acrosome into a comprehensive scheme by indicating that the acroblasts of Lepidoptera, and possibly the acrosome generally, belongs to the Golgi apparatus. The acroblasts are at first Golgi rods, of the typical crescent shape, and with the typical relation to the mitotic spindle. In the later stages they become vesicular, and appear to unite to produce the acrosome as described. In Molluscs (Gatenby, 1917 $b, 1918 a$ ) the Golgi rods of the spermatocyte are at first very similar, but in the spermatid, instead of becoming attached to the nucleus at the anterior end of the developing spermatozoon, they arrange themselves around a dense "archoplasmic" mass behind the nucleus, and with it constitute the so-called "Nebenkern." As the spermatid elongates, this Nebenkern, consisting of Golgi rods and archoplasm, passes gradually down the tail and appears finally to be sloughed off. Meanwhile a small acrosome, of unknown origin, appears at the anterior side of the nucleus; by analogy with the Lepidoptera it seems possible that further investigation may show that this is derived from a portion of the Golgi apparatus separated off from the rest. Like the mitochondria, therefore, the Golgi bodies, while possibly deserving the name of cell-organs, appear to be concerned with cell-differentiation, and can hardly be considered as specific structures having an invariable function, since their fate differs so widely in spermatozoa of different animals.

The rather complicated processes which take place in the later stages of spermatogenesis may be summarised as follows. The greater part of the head consists of the nucleus which develops directly from that of the spermatid by a process of contraction and concentration. The acrosome is described in some forms as arising from a mass of denser 
protoplasm known as the idiozome, but in Lepidoptera from rod-like bodies belonging to the Golgi apparatus, which become vesicular, coalesce around the nucleus, and produce the acrosome. The centrosome of the spermatid sinks in to the neighbourhood of the nucleus, grows out a long filament which forms the axial fibre of the tail, while the centrosome itself is the chief constituent of the middle-piece. In the primary spermatocyte, and sometimes earlier, mitochondrial granules appear as a cloud around the nucleus; they multiply, enlarge, become vesicular, and (possibly in consequence of defective fixation) may coalesce into strands. They become passively distributed during cell division, and in the spermatid either unite to form a definite "mitosome," or may remain as a compact group of vesicles. As the spermatid elongates, the mitochondrial mass is drawn out and forms a sheath around the axial filament of the middlepiece and tail. Whether it is carried in to the egg, or becomes absorbed, or, as has been maintained in at least one case, thrown off, appears to vary in different animals. In addition. to the mitochondria, various other bodies have been -described, the nature of which is not less obscure. The chief of these is the so-called "Nebenkern," which seems, like the acrosome, to be derived from the Golgi apparatus and to be closely connected with the "idiozome" of Meves-a body probably identical with GatenBy's "archoplasm." For the fuller elucidation of the origin and fate of these structures, it seems clear that improvements in the technique of fixing and staining are essential. It should be noted that at least both mitochondria and Golgi apparatus also occur in the earlystages of the developing egg and may be very abundant in the mature egg, but our knowledge of the finer details of oogenesis is even more defective than that of the development of the spermatozoon. 
Finally, it should be mentioned that GoLdschmidt (1915) has shown, by growing spermatocytes and spermatids of Moths in artificial cultures outside the body, that the development of the spermatozoon is dependent on the osmotic relations of the cells as controlled by the follicle membrane in which the sperm-cells are enclosed. And he makes the interesting suggestion, supported by some experimental evidence, that the abnormal spermatozoa, constantly found in a number of Moths and Prosobranch Molluscs, are produced by failure of the follicle membrane to provide the necessary conditions for proper development. In these species certain follicles become abnormal at an early stage of the spermatocytes, and all the cells in the follicles develop abnormally. In the molluscs some of the nuclear matter, and in the moths all of it, is extruded giving rise to so-called oligopyrene and apyrene spermatozoa, which are apparently functionless ${ }^{1}$ (cf. Pl. X). They develop chiefly in rather old testes, and in Moths, as Goldschmidt has shown, are especially abundant in hybrids in which the male characters are largely reduced. The explanation that they are due to abnormal conditions consequent upon the failure of the follicle membrane to supply the necessary conditions seems more probable than any of the numerous other speculations that have been advanced.

Before passing on to consider the development of the ovarian egg, a short space may be devoted to some accessory structures in spermatogenesis found in a number of animals. Frequently not all the primitive germ-cells give rise to spermatozoa; some of them are used up in producing accessory cells of various sorts. In mammals, for example, among the spermatogonia which line the seminiferous tubules of the testis, there are placed at intervals Sertoli cells

1 Cf. Meves (1903), KuschakeWitsch (1913), Gatenby (1917 a). 
which have been shown by Montgomery (I II $b$ ) to be derived by a differential division from the primitive spermatogonia (cf. Pl. X). The manner of their differentiation will be described in Chapter XII; their function appears to be to act as nutrient cells for the developing spermatozoa, bundles of which become attached to them. In some insects

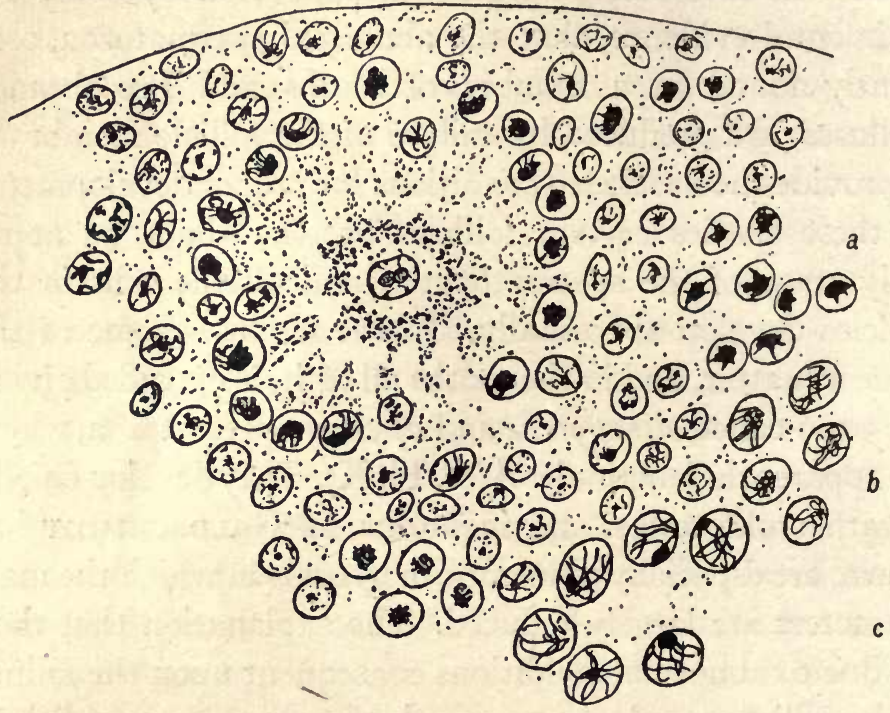

FIG. 1 5. Verson's cell in testis of a butterfly (Pieris brassicae) surrounded by developing spermatocytes.

$a$. Synizesis; $b$. emergence of chromosomes from synizesis.

c. Growing spermatocytes becoming arranged in cysts. (Writer's preparation.)

each compartment of the testis contains a large Verson's cell, around which the spermatogonia are aggregated, and which in the same way appears to serve as a channel for the supply of nutriment to the sperm-cells. In a number of invertebrates the spermatozoa develop in connection with a protoplasmic mass known as a cytophore, from which the spermatogonia are produced by repeated nuclear division, 
giving rise to a large central mass surrounded by a layer of smaller spermatogonia, and later of spermatocytes and spermatids. The cytophore itself is non-nucleated, and seems to serve both for the supply of nourishment and for a point of attachment for the spermatozoa until they are mature. This development of spermatozoa by means of a cytophore is well known in the earthworm, in which the primitive germ-cells are discharged from the testis at an early stage, and the whole of the later spermatogenesis takes place in the seminal vesicles.

\section{OOGENESIS}

The development of the egg in different animals is much less uniform than that of the spermatozoon, in consequence of their relatively great variability in size, structure and amount of yolk in different eggs, and only an illustrative account of some typical features can be given here. An egg before fertilisation is usually a moderately or very large cell containing a variable amount of yolk and enclosed in some kind of membrane. The nucleus before the maturation divisions is commonly large and vesicular, and nearly always has a conspicuous nucleolus; it may be situated either near the centre of the egg, or, especially when there is much yolk, near the surface in a patch of protoplasm free from yolk granules. The egg is often enclosed in a very delicate vitelline membrane, though it may be naked, and outside this there is frequently some sort of outer covering or shell (e.g. the chorion of insects or zona radiata of vertebrates), which may belong to the egg itself or be produced by the surrounding follicle cells. When this outer covering is present before fertilisation, it is usually pierced by a micropyle, a fine canal, at the pole of the egg nearest to the nucleus, by which the spermatozoon enters. Not infrequently 
there is in addition a true shell which is secreted by the oviduct after the egg has left the ovary.

The yolk consists of granules which may be of more than one kind; they may be scattered in the protoplasm with no definite arrangement, or, especially when the yolk is abundant, may be disposed in zones. The yolk-granules of some species stain deeply with chromatin stains, a fact which may occasionally lead to confusion in studying the nuclear divisions in the egg, if care is not exercised. In a variety of animals a body known as the yolk-nucleus ("Dotterkern") has been described, which may persist to a late stage of segmentation as the egg develops. Its function is uncertain and there has been considerable discussion as to its origin and nature. Munson concludes that most of the bodies described as "yolk-nuclei" are in reality the persistent centrospheres of the egg, though the reason for their persistence in this way remains obscure, and further investigation is needed. In a few cases the body is of a different character, and appears to be a true nucleus which has migrated into the egg from one of the surrounding follicle cells.

Some account of the so-called "organ-forming substances" which are present in many eggs will be given in a later chapter.

The chief points that require some consideration in the development of the egg, apart from the history of the nucleus which has already been described, are the deposition of the yolk and the formation of the egg-membranes. In many species no special provision for the supply of yolk to the developing egg seems to be present; the oocytes gradually increase in size in the ovary and as they do so yolk-granules begin to appear in their cytoplasm. In such cases the substances from which the yolk is formed must 
presumably reach the egg by osmosis or by transference from the surrounding cells. In some animals it has been observed (e.g. in Medusae, Echinoderms and Tunicates by SCHAXEL) that during the earlystages of the yolk-deposition chromatin is emitted from the nucleus into the cytoplasm, and it is supposed that this chromatin plays an important part in the formation of the yolk and other substances of the egg. It should be mentioned, however, that other observers have interpreted somewhat similar appearances in the opposite way, and regard the chromatin granules seen in the cytoplasm around the nucleus at various stages of cell-differentiation as chromatin which is being formed, and which will then be absorbed into the nucleus. It has also been maintained that SCHAXEL's granules are not chromatin, but are mitochondrial in nature, and appear, no doubt in connection with the nucleus, at periods of special metabolic activity, for the process only takes place just before substances are deposited in the cytoplasm, as for example in the growing oocyte before the yolk appears, and in the Echinoid mesenchyme when the calcareous spicules are about to develop.

Although the production of yolk without any special external mechanism is not rare, a large number of animals have definite organs or methods for the supply of the yolk to the growing oocyte. One of the commonest of these is by accessory cells generally known as nurse cells, which are often derived from oogonia indistinguishable at first from those which will grow up into true oocytes. These nurse cells supply nourishment to the growing eggs in various ways. A frequent method is by means of an egg-follicle, consisting of an envelope of cells enclosing the oocyte. At first these cells are not conspicuously different from the oocyte itself, especially when they arise from oogonia, but 
gradually the oocyte increases in size while at the same time the follicle cells become smaller, and seem not only to act as channels for the supply of nutriment to the oocyte, but also themselves to be used up in providing it. As was mentioned in the previous chapter, in some insects at least these nutritive cells undergo all the earlier stages of the meiotic phase, and only after reaching the pachytene stage become differentiated from the oocytes which they surround, while at the same time the double chromosomes of their nuclei break up into their univalent components. In insects there are three distinct types of arrangement of the nutritive cells (cf. Text-fig. 8, p. 74). The ovary consists of elongated strands or egg-tubes, with oogonia or young oocytes at the narrow apex, and the older eggs arranged in regular order one behind the other to the lower end, where the tube is continuous with the oviduct. In some insects, for example Orthoptera, the eggs are enclosed in simple follicles, which doubtless supply nourishment to them in their early stages, and secrete the shell or chorion when they become full-sized. In others, for example Coleoptera and Hymenoptera, each egg is separated from the next by a group of nutritive cells, so that the eggs and groups of nurse cells alternate along the tube. Finally, in the Hemiptera there is a single large mass of nutritive cells near the apex of the egg-tube, and all the growing oocytes are connected with this by long strands, along which the nourishment flows from the nurse cells to the egg.

A peculiar condition occurs in Hymenoptera, and possibly some other insects, during the deposition of yolk in the growing egg. Numerous small nuclei appear in the yolk, especially around the edge of the egg, and presumably are concerned with the process of yolk-deposition. GAtEnBY $\mathbf{1}$

1 See also BUChNer, 1918. 
finds that these nuclei arise from minute chromatin granules which increase in size and become vesicular, and that the granules themselves are originally extruded from the eggnucleus at an earlier stage.

A process which is similar in essentials to that found in insects occurs in many Crustacea, in which some oocytes grow at the expense of the rest and completely use them up as they develop into mature eggs. One of the most extreme cases of the kind is seen in the Annelid Opbryotrocha where the eggs develop in the coelom. In this animal each young oocyte has attached to it a single nurse cell, which is at first larger than the oocyte, and has an enormous nucleus; gradually, however, the egg absorbs the substance of the nurse cell, until only a shrivelled vestige of it remains attached to the full grown egg.

Finally, it should be recalled that in the Platyhelminthes the egg itself is without yolk, but the yolk is provided by special glands which open into the oviduct or vagina. The fully formed egg there becomes surrounded by a mass of yolk-cells, and a shell is secreted by glands around the whole group. The "egg" of Platyhelminthes is thus a complex body consisting of a small ovum embedded in yolkcells and the whole enclosed in a shell.

The outer coverings of various eggs differ greatly in different animals, as might be expected in view of the widely varying circumstances in which the eggs begin their development. In a number of marine invertebrates the egg is naked, or is covered only by a soft gelatinous envelope which is easily penetrated by the spermatozoon. Such eggs are generally shed into the water and fertilised by spermatozoa similarly discharged by the male. In Echinoids eggs of this kind secrete a membrane immediately after being fertilised. Many aquatic and most terrestrial animals have 
not only a thin vitelline membrane but also a stronger outer covering. The vitelline membrane is usually if not always part of the ovum, but the outer covering (often called the shell, though a true egg-shell has a different origin) is most frequently secreted by the follicle cells of the ovary, although in several instances, for example the zona radiata of Vertebrates, its origin is uncertain, and it also is possibly produced by the egg itself. Finally in a considerable number of animals a true shell is secreted by glands in the oviduct after the egg leaves the ovary, as, for example, in Elasmobranch fishes, Reptiles, Birds, and among Invertebrates in many Molluscs, some Crustacea, etc. Not rarely there may be an albuminous envelope, either naked or enclosed in the shell, as in Amphibia and Birds. 


\section{CHAPTER VIII}

\section{Fertilisation and Segmentation}

$T^{\text {HE }}$ details of the process of fertilisation are hardly less varied than those of the structure of the egg itself, although its essential features are practically constant throughout the animal kingdom. With rare exceptions the egg is fertilised after it leaves the ovary, but the entrance of the spermatozoon may take place in the oviduct or uterus or after the egg is discharged, according to the species. In some animals the spermatozoon enters before the maturation (polar) divisions of the egg, in others after the first division but before the second, and in others again after both divisions are completed. Frequently only one spermatozoon normally enters each egg, but in a number of animals several constantly penetrate into the egg, although only one conjugates with the egg-nucleus and the rest either degenerate or in Elasmobranchs and Reptiles may form the nuclei of the "vitelline syncytium" around the edge of the germinal disc". The normal entrance of more than one spermatozoon occurs chiefly in eggs rich in yolk, for example in Insects, Elasmobranchs, Reptiles, etc. In eggs which are provided with an outer covering before they leave the ovary the spermatozoon enters by the micropyle, in naked eggs it can often penetrate the surface at any point, though there may be a special region for its reception. It is uncertain whether the spermatozoa are attracted to the egg by some

1 In Bats, according to KohlBRUgGe, several spermatozoa enter the egg, and while one conjugates with the egg-nucleus others fuse with the nuclei of the blastomeres during segmentation. This observation requires confirmation. 
secretion, such as is known to be produced by the archegonia of Mosses and Ferns, or whether they meet the egg by chance; it is usually assumed that the egg exercises some attractive influence, probably of a chemical nature. It is at least certain that the eggs of Echinoderms secrete a substance which greatly increases the activity of the spermatozoa of the same species. It is also known that the spermatozoon will enter a mature egg, or even a nonnucleated fragment of one, in those species in which the maturation divisions normally take place before the spermatozoon enters, while it will not penetrate into an egg which is not yet ready to be fertilised.

When a spermatozoon comes in contact with a naked egg, and sometimes at least when it reaches the egg itself if an outer covering is present, the surface of the egg-protoplasm rises to meet it in the form of a small eminence known as the reception cone (P1. XII, $A-E)$. The formation of this conical projection of the egg-protoplasm is almost certainly a surface-tension phenomenon, due to an alteration of the surface tension at the point where the spermatozoon touches the egg. The head of the spermatozoon is drawn into the protoplasm of the reception cone, and in some cases the tail is separated off; in others the whole spermatozoon enters the egg. In Echinoderms immediately after the spermatozoon touches the surface of the egg a delicate membrane is formed from the egg surface (the so-called vitelline or fertilisation membrane); its production begins in the region immediately around the point of entrance of the spermatozoon, but rapidly travels over the surface of the egg, so that in an extremely short time the egg is enclosed in a membrane-which is separated by a space containing fluid from the surface of the egg. The formation of the fertilisation membrane of Echinoderms is also 
almost certainly a surface-tension phenomenon, and can be induced in unfertilised eggs by certain reagents such as are used to bring about artificial parthenogenesis ${ }^{\mathbf{1}}$.

Although it is often said that the fertilisation membrane has the function of preventing the entrance of more than one spermatozoon, it does not provide the only means by which polyspermy is prevented, as is proved by the fact that if a fertilised egg is cut in two, and the membrane destroyed, the halves do not allow the entrance of additional spermatozoa, while these readily enter the halves of an unfertilised egg. This change in the condition of the egg appears to arise from the influence exerted by the centrosome which is introduced by-or at least formed in connection with-the middle-piece of the spermatozoon. As has been described in Chapter IV, around the centrosome an aster develops. When several spermatozoa enter an egg simultaneously, as may happen when the sperm is concentrated, or if the egg has been treated with an anaesthetic, an aster develops in connection with each, and these asters appear to repel one another so as to divide the protoplasm of the egg among them, without any overlapping. Each centrosome and aster, in fact, seems to take possession of the area of protoplasm around it, and make it impenetrable either to another spermatozoon or to the rays of. another aster. In an ordinary monospermic egg the same thing happens; the single centrosome "takes possession" of the whole egg and it then becomes impenetrable to any

1 Some investigators maintain that the membrane is not formed at fertilisation, but that a pre-existing membrane is separated from the egg by a "wave of contraction" accompanying the expulsion of waste substances from the egg consequent on the stimulus and resulting change of permeability induced by the entrance of the spermatozoon. The membrane after fertilisation, however, must have a different consistency, for it is then impermeable to spermatozoa. For a discussion of this subject see BraCHET (1917).

D.C. 
later spermatozoa. It has also been maintained that the fluid between the egg and the membrane has an agglutinative effect upon spermatozoa, and inhibits the movement of any which may have penetrated within the membrane.

In a number of animals only the head and middle-piece of the spermatozoon enter the egg; in others the whole spermatozoon penetrates into it, but the tail soon breaks off. The head and middle-piece then rotate, so that the middle-piece precedes the head as they sink deeper in to the egg substance. Round the middle-piece radiations then begin to appear in the egg-protoplasm, and the middle-piece is either converted into the centrosome which will give rise to the spindle of the first segmentation division, or, according to some investigators, the centrosome arises from the interaction of the middle-piece and the egg cytoplasm (Pl. XII, $B-I)$.

From this stage onward the details of the process of fertilisation differ somewhat according to whether the egg is one of those which undergoes its maturation-divisions before or after the entrance of the spermatozoon. If the maturation-divisions have already taken place before the spermatozoon enters, as for example in Echinoids, the eggnucleus is ready immediately for fertilisation. In such eggs the sperm head, preceded by the middle-piece or developing centrosome, sinks rapidly into the egg, at first towards the centre, and then, if the egg-nucleus is excentric, towards the egg-nucleus; as the sperm head approaches, the egg-nucleus also begins to move to meet it. Especially in yolky eggs, the sperm head, as it sinks into the egg, leaves behind it a definite track in the egg substance, so that the course which it has followed remains visible.)

The nature of the attraction between the two nuclei is quite unknown. As it sinks in, the sperm head begins to 
swell, becones vacuolated, and gradually assumes the form of a nucleus. In eggs in which the egg nucleus is ready for fertilisation before the spermatozoon enters, the sperm nucleus (sometimes called the "male pronucleus") often reaches the egg nucleus before it has reached its full size, and in this case the two nuclei come into contact and unite to form a single large zygote nucleus. If the sperm nucleus is still small as compared with the egg nucleus this conjugation may have the appearance of the small sperm nucleus being absorbed into the larger egg nucleus. In such eggs the development of definite chromosomes only takes place after the union of the two nuclei, and they then appear, of course, in the double (somatic) number, half being derived from each parent. The centrosome derived from the middlepiece of the spermatozoon also only divides after the two nuclei have come into contact, and the spindle of the first segmentation division arises when the membrane of the zygote nucleus disappears (Pl. XII, $\mathcal{F}, K, L)$.

In a large number of animals the polar divisions of the egg only take place, or are only completed, after the spermatozoon enters it. Sometimes the whole of the maturation division is subsequent to the entrance of the spermatozoon; often the first polar spindle is already developed, but the entrance of the spermatozoon seems to provide the necessary stimulus for the completion of that division and for the formation of the second polar body. In other species, again, the first polar body has already been formed but the second division occurs only when the spermatozoon is present. In all these cases the egg nucleus is not ready for conjugation when the spermatozoon penetrates the egg surface, and the subsequent behaviour of the spermatozoon is therefore somewhat different from that described above. In eggs of this kind the spermatozoon penetrates for some. 
distance into the egg and then stops; there appegrs to be no attraction between it and the egg nucleus at this stage, and it waits until the egg nucleus is ready. As it does so it increases in size until it becomes a large vesicular nucleus, and the chromatin separates out into definite chromosomes which appear in the reduced number. The centrosome, derived from the middle-piece, meanwhile divides, and between the two centrosomes so produced a spindle arises in the neighbourhood of the sperm nucleus. While these changes have been in progress in the spermatozoon, the egg nucleus has been completing its maturation, and when this has happened the mature nucleus shows a definite attraction for the sperm nucleus, and begins to travel towards it. The two nuclei, now alike in size and appearance and each containing the reduced number of chromosomes, thus come together, usually from opposite sides of the spindle formed between the two centrosomes, and as they come into contact their membranes dissolve and they give rise to two groups of chromosomes, which arrange themselves in the equator of the spindle. In these cases, therefore, no complete conjugation of the egg and sperm nuclei as such takes place; the two nuclei break down into groups of chromosomes before uniting, and the intermingling of the paternal and maternal chromosomes takes place only in the anaphase of the first segmentation division (cf. Pl. III). In some animals, for example in Copepods, it has been described as taking place even later, for the two groups of chromosomes travel to the poles separately, and these give rise to nuclei which are still recognisably double, so that the paternal and maternal portions of each of the first two segmentation nuclei are still quite distinct (cf. Pl. XXII, lower series $a, b$ ).

It is clear, however, that the differences between the two 
types of fertilisation are all differences of detail. In essentials the process of fertilisation is uniform throughout the animal kingdom; it consists in the coming together of two nuclei derived from the two parents, and the mingling and division of their substance in such a way that the chromosomes from each parent are equally represented in both the daughter nuclei produced by the first segmentation division.

Some discussion of the relation of fertilisation to the initiation of development in the egg will be given in connection with the subject of Artificial Parthenogenesis in a later chapter. It will be convenient, however, here to devote a few lines to the early segmentation divisions which normally follow immediately after the conjugation of the sperm and egg nuclei. The zygote nucleus as has been said, divides immediately, but the nature of the division of the cell varies greatly according to whether the egg is poor or rich in yolk. In an egg with relatively little yolk, as for example in Mammals, Amphibia, Echinoderms and many other Invertebrates, a complete cell-division takes place immediately after the nuclear division, separating the egg into two blastomeres which are only loosely connected with each other. These two blastomeres are usually of equal size, though in some forms one is large, containing most of the yolk, and the other small. The position of the first cleavage plane nearly always has some definite relation to the orientation of the parts of the future embryo, and most frequently corresponds with its sagittal plane, dividing the egg into right and left halves. It is evidently of importance, therefore, to discover what determines the position of this first cleavage plane, and whether it depends on the structure of the egg alone, or may be affected by the spermatozoon. The same problem arises in eggs in which the cleavage is 
partial, as happens when a large amount of yolk is present, at least in the case of telo-lecithal eggs such as those of Vertebrates and Cephalopods, in which the greater part of the protoplasm is at one pole and in which the cleavage furrows divide the polar cap of protoplasm but do not extend far into the yolk. The problem in centro-lecithal eggs, characteristic of Arthropods, is somewhat different, and will be considered later.

In many eggs in which the first cleavage plane has a definite relation to the position of parts in the future embryo it is undoubtedly true that this orientation of the embryo depends on the pre-existing structure or polarity of the egg, and in these the position of the first cleavage plane is also determined by the same causes. In a number of eggs, however, there is no such complete predetermination. It is maintained by DRIESCH and others that eggs of this type are equipotential systems, that is to say, they are not differentiated before fertilisation into regions of different potentiality, and any part of the egg is capable of giving rise to any part of the embryo. It is doubtful whether any egg exists which is not to some extent differentiated into different regions, but experiments in separating blastomeres after the first or second cleavage division show that in a number of eggs each of the first four blastomeres can give rise to a perfect larva, and that therefore the differentiation of parts in the embryo cannot be due solely to pre-existing arrangements of "organforming" substances in the egg. Eggs of this type bring to out notice in a striking manner one of the fundamental problems of biology, the problem of the differentiation by division of a cell in which the parts are equipotential into an embryo in which the parts are conspicuously different. Most commonly, under natural conditions, the first step of this process is the division of the egg into two blastomeres 
which will form the right and left halves of the embryo, although experiment shows that in eggs of this kind each of these blastomeres has in it the potentiality of producing a whole embryo if they are artificially separated. What, then, determines the position of the first cleavage plane? The plane of cell-division is always perpendicular to the axis of the mitotic spindle, and this in turn depends on the position of the centrosomes. In an egg which is truly equipotential there would appear to be only one factor which could determine what position the centrosomes shall take up; this factor is the entrance path of the spermatozoon. As the sperm nucleus travels towards the egg nucleus the centrosome derived from the accompanying middle-piece divides and the daughter centrosomes diverge in a line at right angles to the entrance path; since, therefore, the plane of cytoplasmic division is at right angles to the line joining the centrosomes it must coincide with the entrance path of the sperm nucleus, if this is straight, and even if it is curved the division plane has a definite relation to it. In the majority of eggs there is a definite polarity, but all meridians running through the two poles are alike, and in eggs of this type, as was shown by Roux in the Frog, the first cleavage plane runs through the poles, but its meridian is usually determined by the path of entrance of the spermatozoon. Subsequent investigators (BRACHET and others) found that in the Frog the first cleavage plane does not always coincide with the sagittal plane of the embryo; in about 70 per cent. of the eggs it does so, but in the remainder it is either at right angles to it or inclined to it at a varying angle, often approaching $45^{\circ}$. Although, however, the first cleavage plane is variable in position, the entrance path of the spermatozoon nevertheless always determines the plane of bilateral symmetry of the embryo, for the substances of 
the egg undergo re-arrangement immediately after the entrance of the spermatozoon in such a way as to give the egg a bilateral symmetry, and the meridian of symmetry always coincides with the subsequent sagittal plane of the embryo, whether the first cleavage plane does so or not. Roux showed that although the first cleavage plane of the Frog usually includes the entrance path of the spermatozoon it is easy to alter this arrangement by compressing the egg in a glass tube, in accordance with the law of cytoplasmic division mentioned in Chapter III, and presumably in the relatively small proportion of naturally developing eggs in which the two planes do not coincide, the re-arrangement of the materials of the egg consequent on the entrance of the spermatozoon takes place in such a way as to involve an alteration in the position of the first cleavage spindle similar to that induced by compression.

In the second and later segmentation divisions the arrangement of the cleavage planes depends on the law just referred to- that the spindle takes up its position in the longest axis of the cell-protoplasm as distinct from yolk. In eggs in which there are materials of different sorts, these become segregated sooner or later into separate blastomeres or groups of blastomeres, and it is pointed out by BRACHET that during segmentation there is no true cell-differentiation, in the sense of the production of new structures in some of the cells, but only "a cutting up of the egg into smaller and smaller territories ${ }^{1}$ " which differ from one another in including different parts of the original egg materials. The relation of this segregation of the egg materials into separate blastomeres to true cell-differentiation will be referred to in a later chapter. Apart from its

1 BRACHET, 1917 , p. 259. 
connection with differentiation of parts, the actual pattern or arrangement of the blastomeres in a segmenting egg is a matter of interest. It appears that this follows the law of the minimal area of surface contact, according to which any drops of liquid are compelled by surface-tension to arrange themselves so that the sum of their surfaces is reduced to a minimum. Where this law appears to be broken, the cause is probably to be sought in the different consistency of different parts of the egg 1 .

All the examples of segmentation mentioned hitherto have been drawn either from eggs having total cleavage, or from telo-lecithal eggs in which a polar blastoderm is formed. Especially among the Arthropods a different type of cleavage is found, known as centro-lecithal. In these eggs the yolk forms a central mass and is enclosed in a sheath of yolk-free protoplasm which extends over the whole surface of the egg, though it is usually thicker in one region than elsewhere. In eggs of this type the conjugation of the egg and sperm nuclei usually takes place deep in the yolk and the division of the zygote nucleus is not followed by any immediate cleavage of the egg protoplasm. The zygote nucleus divides, its two daughter nuclei separate and divide again, and the process is repeated until a number of nuclei are scattered through the yolk. Then some or all of them migrate to the surface and enter the superficial layer of protoplasm, which thus becomes a blastodermic epithelium in which the cells are more or less clearly marked. In these eggs there appears to be no necessary relation between the position of the first division-figure and the orientation of the embryo. The position of the various parts of the embryo is determined by a definite polarity of the egg itself, and the subsequent fate of each of the numerous segmentation 
nuclei depends on the region of the outer protoplasm with which it happens to be associated when it comes to the surface at the formation of the blastoderm.

One other point of some interest in connection with cleavage remains to be mentioned. In developing eggs in which the blastomeres are all approximately equal in size, all the cells divide simultaneously. When there is much yolk in some of the cells, or after visible differentiation into cells of different sizes has begun, the larger cells begin to lag behind the smaller ones, but in some eggs, even many hours after segmentation has begun, the simultaneity of division is very striking. In Insects, for example, it persists until the blastoderm stage; an egg may often be found in which all the cells of the blastoderm are not only dividing, but are in the same stage of division. This may be due simply to the fact that all the cells, being similar, develop at the same rate, but the possibility of mutual influence upon one another must not be overlooked. That the blastomeres do influence one another is clearly shown by the fact that in an equipotential egg each of the first two or four cleavage cells, if isolated, will produce a complete embryo, though when associated with its fellows it gives rise only to one half or one quarter. The association and mutual position of the blastomeres is thus an essential condition for the differentiation of parts of the embryo.

Although not very nearly connected with the preceding subjects, it will be convenient to give here some account of the phenomenon of polyspermy, that is, of the abnormal fertilisation of the egg by two or more spermatozoa, to which reference has already been made in earlier chapters. In Echinoid eggs the entrance of a spermatozoon normally not only induces the almost instantaneous secretion of a "fertilisation membrane," but also very quickly renders 
the egg-surface impermeable to other spermatozoa even if the membrane be broken. If, however, the vitality of the egg is impaired by treatment before fertilisation in various ways (e.g. with certain alkaloids, heat, or long standing in water) the formation of the membrane is delayed and more than one spermatozoon may enter, and in consequence a more or less abnormal fertilisation process takes place. The simplest and commonest case is that when two spermatozoa are present in the egg. When this happens both spermatozoa give rise to nuclei and centrosomes, and usually each centrosome divides, with the result that at the first segmentation division there are four centrosomes and three times the reduced number of chromosomes. Not rarely one of the centrosomes fails to divide, so that there are three instead of four. In the first case the division figure takes the form of a tetraster, a figure having four poles with a spindle arranged between each and the next, forming roughly the sides of a square; some observers maintain that there are also diagonal fibres running across the middle of the square from corner to corner; others deny this. When there are only three centrosomes the figure is a triaster consisting of three spindles arranged in a triangle (cf. Text-fig. 6, p. 57). The chromosomes become arranged on the spindles rather irregularly, and some may be omitted altogether and remain lying in the centre. Those which take their places on the spindles divide normally, and their halves pass to the poles in the usual way, thus giving rise to three or four nuclei near the centrosomes. The egg then divides simultaneously into as many blastomeres as there are nuclei, and development may proceed apparently normally for a considerable period, though in the later embryo and larva abnormalities always appear.

A number of interesting problems centre round these 
multipolar spindles in dispermic eggs, some of which have already been referred to and others will be mentioned later. Among other things, they have figured largely in the discussion of HARTOG's hypothesis of a dual force acting at unlike poles as the cause of mitotic division. It is impossible here to enter into HARTOG's ingenious explanation of multipolar spindles on his hypothesis; it must suffice to say that he maintains that they are in no way inconsistent with it. 


\section{CHAPTER IX}

\section{Parthenogenesis}

$T^{N}$ the great majority of animals reproduction is biparental-new individuals arise from a zygote formed by the union of an egg and a spermatozoon. There are, however, at least three other methods of reproduction which are widespread among the lower animals and found to some extent even in some of the "higher." These are fission, budding, and parthenogenesis. Fission is of course a common method of reproduction among the Protozoa, but is not by any means confined to them; it occurs in the adults of some Coelenterates and Echinoderms, and embryonic fission is found in certain Polyzoa and Insects. Budding is not quite easy to separate sharply from fission; they grade into one another, though usually the two processes are sufficiently distinct. Parthenogenesis belongs to a rather different category, though at times it is possible to confuse it with budding. It may be defined as the production of a new organism from an unfertilised egg, and if a bud should arise from a single cell, it is evident that the decision as to whether the process is or is not parthenogenesis would depend on whether the cell were or were not an egg. Usually there is no difficulty; in a few cases, as in the paedogenesis of the Cecidomyid flies, some observers have maintained that what is in fact true parthenogenesis is internal budding.

True parthenogenesis occurs in nature under a number of different circumstances. Most frequently it alternates more or less regularly with bisexual reproduction; in only very few species is it the only method of reproduction known. 
The manner of the alternation, however, varies greatly. In some species, for example, many of the Hymenopterous Gall-flies (Cynipidae), parthenogenetic and sexual generations succeed each other quite regularly-there are two generations in the year and one is always parthenogenetic and the other bisexual. More commonly the alternation is less regular, and several parthenogenetic generations intervene between each bisexual generation, as in most Aphids, Daphnids and Rotifers; or again in other members of the same groups there may be no regularity, and one individual female may at one time lay parthenogenetic, at another fertilised, eggs. Finally, in some species parthenogenesis may be "facultative," that is to say, the eggs are capable of being fertilised but develop equally well parthenogenetically; this condition is best known in the Hymenoptera, for example in the Bee and in various members of other Hymenopterous families. In all these cases it is the rule that fertilised eggs produce only females; in most Hymenoptera unfertilised eggs produce only males, and in other groups and in some Hymenoptera, either males and females, according to the nature of the maturation divisions. To this question of sex-determination further reference will be made later.

It has been seen in the chapter dealing with the maturation of the egg that this process normally involves the reduction of the chromosomes to half the somatic number, the full number being restored at fertilisation by the union of the egg and sperm nuclei. Evidently, then, some modification of the process is to be expected in eggs which are destined to develop parthenogenetically, for otherwise these eggs would give rise to individuals having only the reduced number of chromosomes, unless some process of doubling occurred later. And, in fact, it is found that various 


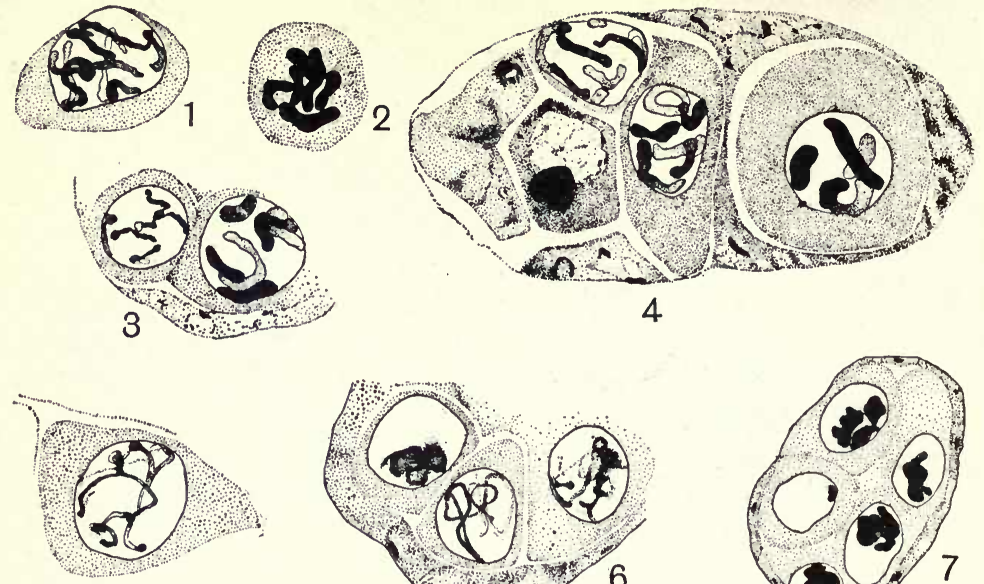

5
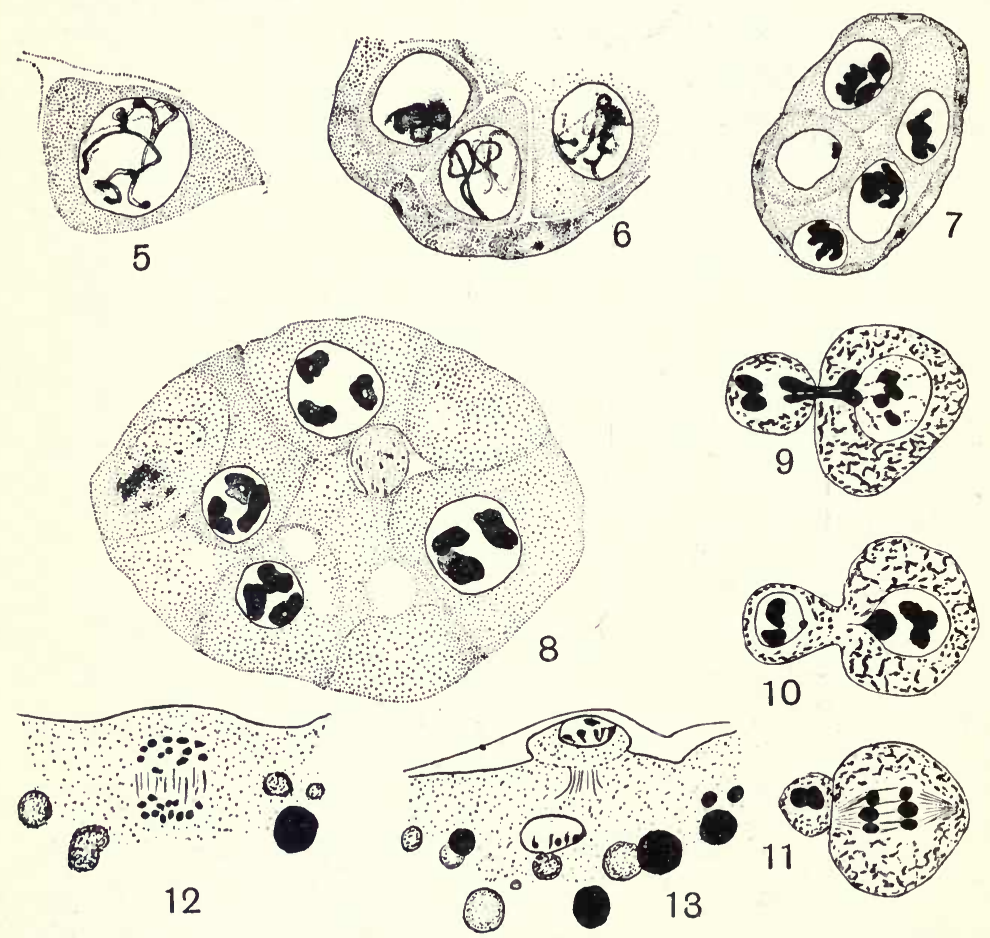

12

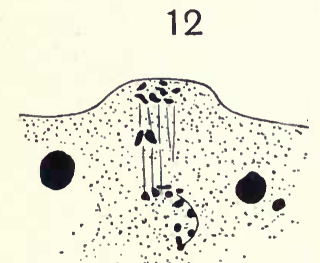

14

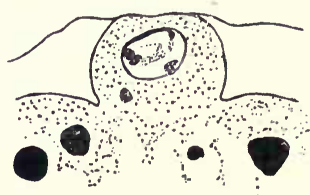

15

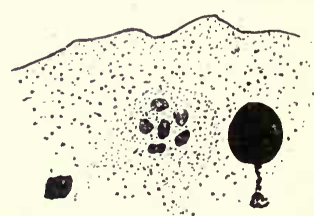

16

Oogenesis and spermatogenesis in Aphids, after Morgan (19 I 5).

1-8. Oogenesis of Phyllaphis.

I. Prophase, 2. Metaphase, of oogonial division, with six chromosomes. 3 , 4, Young parthenogenetic ova with six chromosomes. 5, 6, 7. Synizesis stages of sexual ova. 8. Young sexual ova after synizesis with three double chromosomes.

9, I $\circ$ I I. Three stages of spermatogenesis. 9, r 0 . Unequal first spermatocyte division. I r. Equal division of the larger secondary spermatocyte.

1 2-16. Polar divisions of eggs of Phylloxera fallax.

12, I3. Anaphase and telophase of the single polar division of the female producing egg. 
modifications of the maturation divisions of parthenogenetic eggs do occur, the purpose of which, if one may speak thus teleologically, is to obviate this difficulty. The maturation processes vary in different cases, and it will be convenient to classify them under several heads.

(I) Maturation with one polar division: no chromosome reduction.

This may be regarded as the most typical mode of maturation of parthenogenetic eggs; it is found in the parthenogenetic Crustacea (Phyllopoda, Ostracoda), in the Aphids, in the paedogenetic ova of the Cecidomyid flies, and probably in at least the female-producing eggs of Rotifers. Although this type of maturation occurs in so many species, the details have been worked out in comparatively few, and there is room for much further investigation before our knowledge can be complete. It has long been known that in members of the groups mentioned the parthenogenetic eggs give off only one polar body, while the eggs that require fertilisation produce two, and it is a matter for surprise that more detailed work on the subject has not been undertaken. One of the most complete papers on the subject is that by Morgan (1915) on Phyllaphis coweni and otherspecies (Pl.XIII). In Pbyllaphis the somatic number of chromosomes is six. In the oogenesis of the fertilisable eggs there is a typical contraction-phase (synizesis) from which emerge three doublechromosomes; the eggs undergo a typical double maturation division, giving off two polar bodies, and leaving three single chromosomes in the nucleus. In the development of the parthenogenetic eggs, on the contrary, there is no synizesis, six chromosomes are clearly visible in the growing oocytes, and the egg undergoes only one polar division, leaving six chromosomes in the mature egg-nucleus. The occurrence of synizesis in the oocytes of parthenogenetic 
forms seems to be variable. In the parthenogenetic Ostracoda (Crustacea), Woltereck (1898) and Schleip (I908) have described a typical synizesis in the development of parthenogenetic eggs, although the chromosomes emerge from it in the somatic instead of in the reduced number. In Artemia, however, FrIEs found no synizesis, although this stage is represented in a typical manner in the nearly related Brancbipus, the eggs of which are fertilised, and KÜHN finds no definite synizesis in the summer eggs of Daphnids. There appears, therefore, to be no general rule with regard to the presence or absence of the synizesis stage in the oogenesis of forms in which the parthenogenetic eggs give off only one polar body, but all observers agree that these eggs contain the somatic number of chromosomes, and that the reduction division is absent. In those eggs which go through the synizesis stage, either this is unconnected with the pairing of the chromosomes, or, if pairing occurs, it is of a temporary nature, and the paired chromosomes break apart permanently as they do temporarily in Lepidosiren. The very interesting problems connected with the differences between the male- and female-producing eggs in animals having this type of parthenogenesis must be left for consideration in a later chapter.

A subdivision of the class of parthenogenesis described above is that in which the eggs which will give rise to females undergo no reduction division and produce one polar body, while those which yield males have a typical reduction division and two polar bodies. This type, confined, so far as is known, to the Rotifers, will be referred to again in the chapter on the cytological basis of sex-determination (Chapter XI).

(2) A second, and very rare, type of maturation process in eggs which develop unfertilised was first described by 
Brauer in the Phyllopod Artemia, and has subsequently been observed in a few other cases. BRAUER stated that in some eggs of Artemia, instead of the usual production of only one polar body without reduction, two polar divisions took place, presumably with reduction of chromosomes. The second polar nucleus, however, instead of being thrown out of the egg, remained in it, and after a time sank back from the surface of the egg towards the egg nucleus and the two conjugated exactly as the sperm nucleus conjugates with the egg nucleus of a fertilised egg. The polar nucleus thus took the place of a sperm nucleus, and the egg may be said to be fertilised by one of its own polar nuclei. Confirmation of BRAUER's statement was for a long time lacking, and doubt was cast upon its correctness, although investigations intended to settle the matter showed the existence of other curious facts in Artemia. For example, Аrтom finds that a race from Capodistria on the Adriatic has a somatic number of 84 chromosomes and is always parthenogenetic, while the race found at Cagliari in Sardinia has 42 chromosomes in its somatic nuclei, which are consequently half the size and twice as numerous, and this race always reproduces sexually. Subsequently to BRAUER's paper other observers have discovered a similar fertilisation by the second polar body in two or three cases-by BRUEs in Xenos, an insect of the family Stylopidae, and Buchner (I9I I) in eggs of a starfish made to develop parthenogenetically by treatment with carbon dioxide, while more recently Miss Morris has made similar observations on the eggs of the Mollusc Cumingia caused to develop parthenogenetically. Among plants also a similar process has been described by Rosen Berg in Hieracium, so that the phenomenon of fertilisation by the second polar nucleus may be regarded as established, at least in rare cases.

D. c. 
(3) Maturation with two polar divisions, giving rise to three polar nuclei and an egg nucleus.

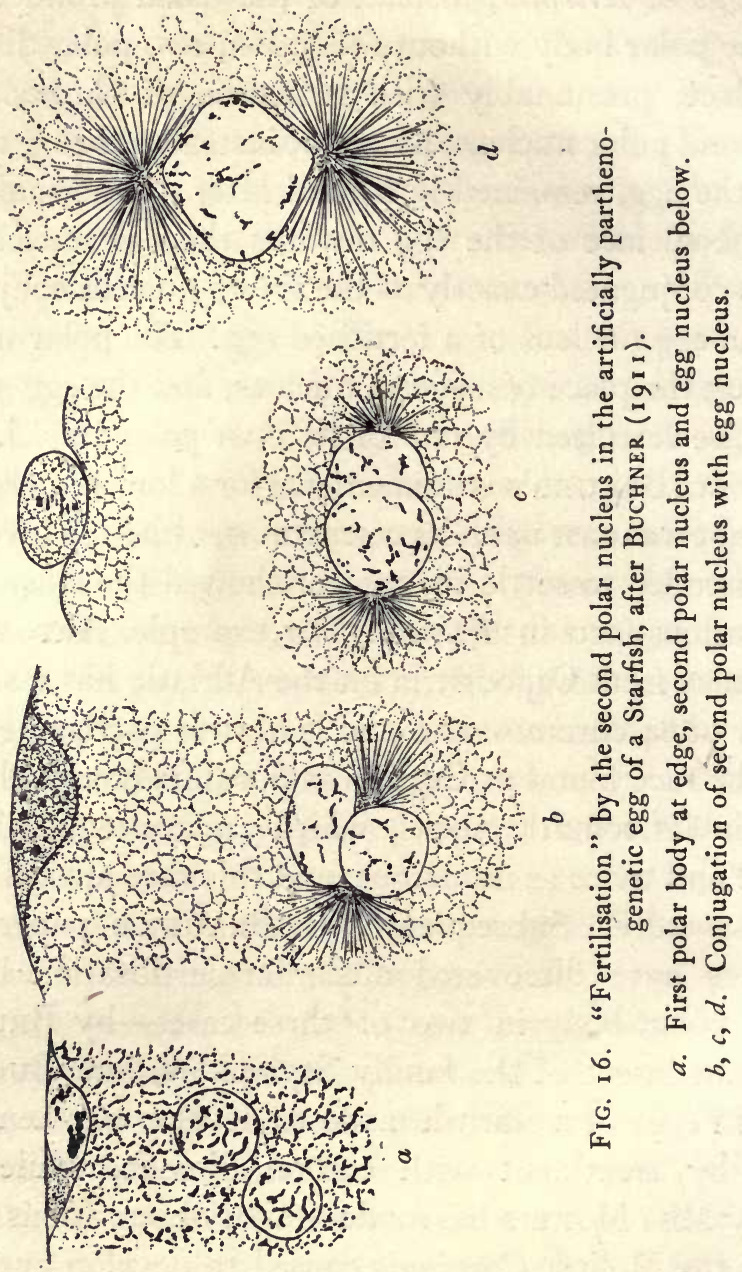

The maturation of parthenogenetic eggs with two polar divisions appears to be confined to the Insects ${ }^{1}$, and is best

1 Apart from the male-producing eggs of the Rotifers mentioned in group ( $\mathrm{I}$ ) above. 
known in the Hymenoptera. This method can be subdivided into two sub-classes, of which the first is well known while the second is somewhat doubtful, and requires further investigation before it can be regarded as substantiated with certainty. The first sub-division includes a large number of Hymenoptera, of several families, in which the polar divisions are undoubtedly reducing, so that the mature egg nucleus contains half the somatic number of chromosomes. These eggs may then be fertilised or develop parthenogenetically; if fertilised they produce females, if parthenogenetic, males. In this group, therefore, the parthenogenesis is of the kind known as facultative. The second subdivision consists in the not very well established case of two polar divisions each of which is equational, resulting in the production of an egg nucleus containing the somatic (double) number of chromosomes. This condition has been described in certain Sawflies (Tenthredinidae) and Gall-flies (Cynipidae) among Hymenoptera, in the stick insect Bacillus (probably) among Orthoptera, and perhaps exists also in the Moths in which unfertilised eggs occasionally undergo development. The nature of the maturation process in certain other parthenogenetic insects, for example the Thysanoptera ( $($ hrips), is unknown.

In the Hymenoptera the maturation divisions have been investigated in a number of species belonging to several families, and with the exceptions to be mentioned later they generally conform rather closely to a common type. The early stages leading up to the maturation divisions take place in the oocytes before the yolk is deposited, and appear to be typical, giving rise to the reduced number of double chromosomes (pseudo-reduction). During the deposition of yolk the nucleus is large and vesicular, with scattered chromatin, but when the egg is fully formed the nucleus 
becomes very small and moves to the edge of the egg near the anterior pole, where there is a thickening of the outer protoplasmic envelope free from yolk. Spermatozoa are received by the female at copulation into the receptaculum seminis, and in some species, for example, the Bees and Wasps, it appears that the female is able to control the fertilisation of the egg; that is to say, she can "at will" allow an egg as it is laid to receive spermatozoa, or withhold them. In other species the females lay equally readily whether they have copulated or are virgin. The maturation process is not affected by the presence or absence of spermatozoa; immediately after the egg is laid the egg nucleus breaks down, a spindle is formed vertical to the surface of the egg, and the chromosomes divide and pass to the poles. They may there form daughter nuclei, but more often two new spindles arise immediately, one below the other, with the two groups of chromosomes from the first division in their equatorial planes. These chromosomes then divide again, and pass to the poles, giving rise to four groups in a line vertical to the egg surface. The outermost group is just below the surface, the two middle ones are close together, at the inner end of the outer spindle and the outer end of the inner, and the innermost group, which forms the mature egg nucleus, is rather deep in the egg (cf.Text-fig.9, p. 8I). In some species each group forms a vesicular nucleus; in others the three outer, or polar, groups remain as little clumps of chromosomes, while the innermost sinks inwards and forms the egg nucleus. In either case it is common for the two middle nuclei or chromosome groups to unite; when they are vesicular nuclei they may appear to conjugate just as an egg and a sperm nucleus do, and then break down into chromosomes which may undergo an irregular division. It has been maintained that in the Bee this false zygote-nucleus 
persists and ultimately gives rise to the genital cells of the larva, but this has been shown to be erroneous. Sometimes all three groups of polar chromosomes unite into a single mass, and Silvestri has shown that in certain parasitic Hymenoptera of the families Chalcididae and Proctotrypidae this united mass gives rise to a nucleus which later divides and provides the nuclei for the envelope which encloses the embryonic mass. With rare exceptions this only happens in species which have embryonic fission; in those species in which each egg gives rise to only one embryo, the polar nuclei or chromosomes sooner or later degenerate completely (cf. Fig. 10, p. 82).

Development proceeds similarly whether the egg has received a spermatozoon or not. If spermatozoa are present, one of them conjugates with the egg nucleus, so giving rise to the somatic (double) number of chromosomes. If the egg is unfertilised, the egg nucleus segments normally, but, at least in the earlier divisions, only the reduced number of chromosomes is present. The embryo derived from a fertilised egg develops into a female, that from an unfertilised egg into a male. The male thus starts life with only half the normal number of chromosomes, and this condition persists throughout its life in the germ-cells and, at least in some species, in the cells of the nervous system. In other tissues, by a process which has not been sufficiently investigated, the number becomes doubled, or in some cells even quadrupled, so that in most cells of the body the male and female have the same number of chromosomes.

Since the chromosomes are present in the reduced number in the spermatogonia before maturation, it is evident that their number cannot be again halved in the spermatocyte divisions, and it is found in all the Hymenoptera in which the spermatogenesis has been followed that one of 
the spermatocyte divisions is suppressed. This was first discovered by Meves (1907) in the Hive Bee; it has since been observed in other bees, and in Wasps, Sawflies and Gall-flies. In most species the spermatocytes undergo only one division, in which the chromosomes divide equationally, so that the spermatids contain the same number of chromosomes as the spermatogonia. Traces of a first division may occur in the formation of a spindle in which the chromosomes do not divide, followed by the separation of a minute piece of cytoplasm containing one of the centrosomes (Pl. XIV). In the Hive Bee a curious and at present unexplained modification of this process is found ( $\mathrm{Pl} . \mathrm{XV})$. After a first abortive division, in which a small piece of cytoplasm is separated from the spermatocyte, a fresh spindle is formed; one pole of this spindle extends into a long finger-like projection of the cell, at the apex of which is the centrosome. The chromosomes divide, and one group passes into the projection and forms a vesicular nucleus, while the other group forms a nucleus in the body of the cell. An extremely unequal cell division then occurs, by which the nucleus in the narrow projection is separated almost without any accompanying cytoplasm from the rest of the cell. The cell, now a spermatid, proceeds to develop in the normal way into a spermatozoon, while the extruded nucleus, after passing through similar stages for a time, gradually degenerates. No adequate explanation of this "polar-body formation" by the spermatocytes of the Hive Bee has yet been given, and in other Hymenoptera, including the Humble Bee, the spermatocytes divide equally (cf. Pl. XIV).

In all the species mentioned hitherto under this heading the eggs which develop parthenogenetically give rise to males; this is sometimes called arrbenotokous partheno- 


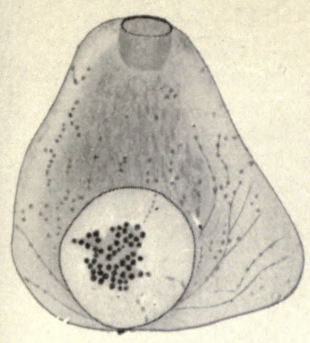

( I)

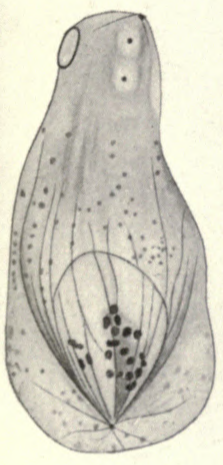

(4)

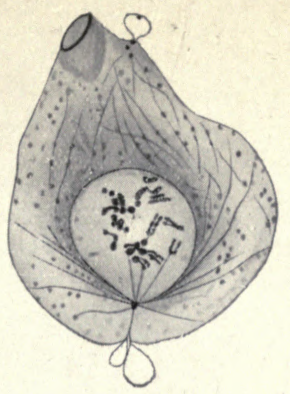

(2)

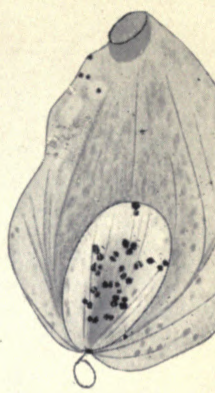

(3)

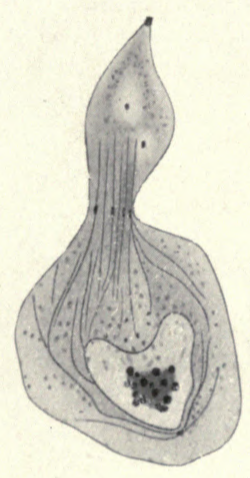

(5)

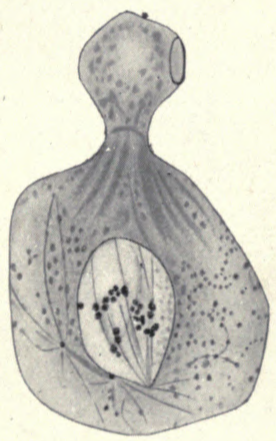

(6)

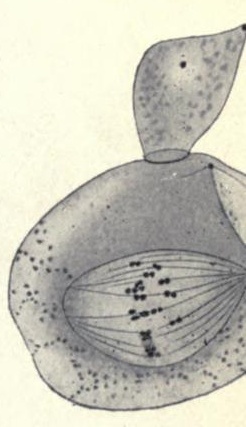

(7)

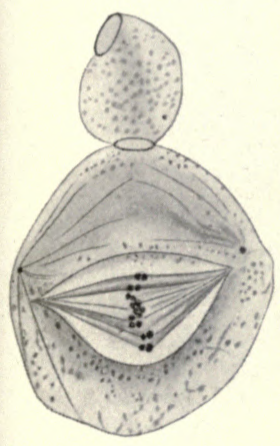

(8)

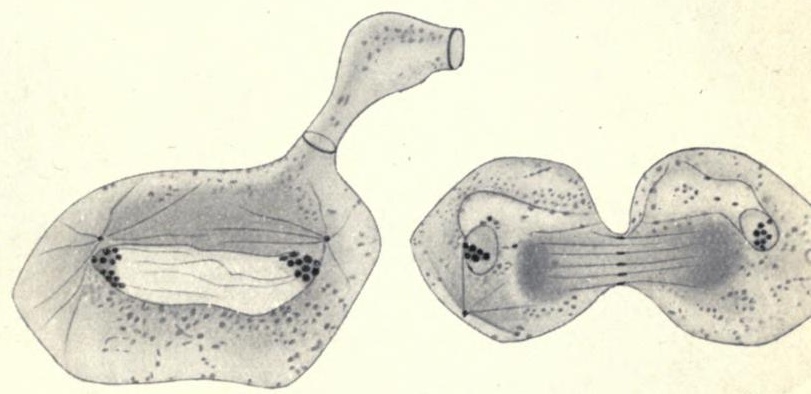

(9)

( 10$)$

Spermatogenesis of the Hornet (after Meves, from the Author's Determination of Sex). (1) Sperm mother-cell. (2-6) Abortive division of the nucleus, resulting in the separation of a piece of protoplasm above, but leaving the whole nucleus in the larger (lower) cell. (7-10) Normal division of the large cell into two daughter-cells, each of which gives rise to a spermatozoon. 

Plate XV

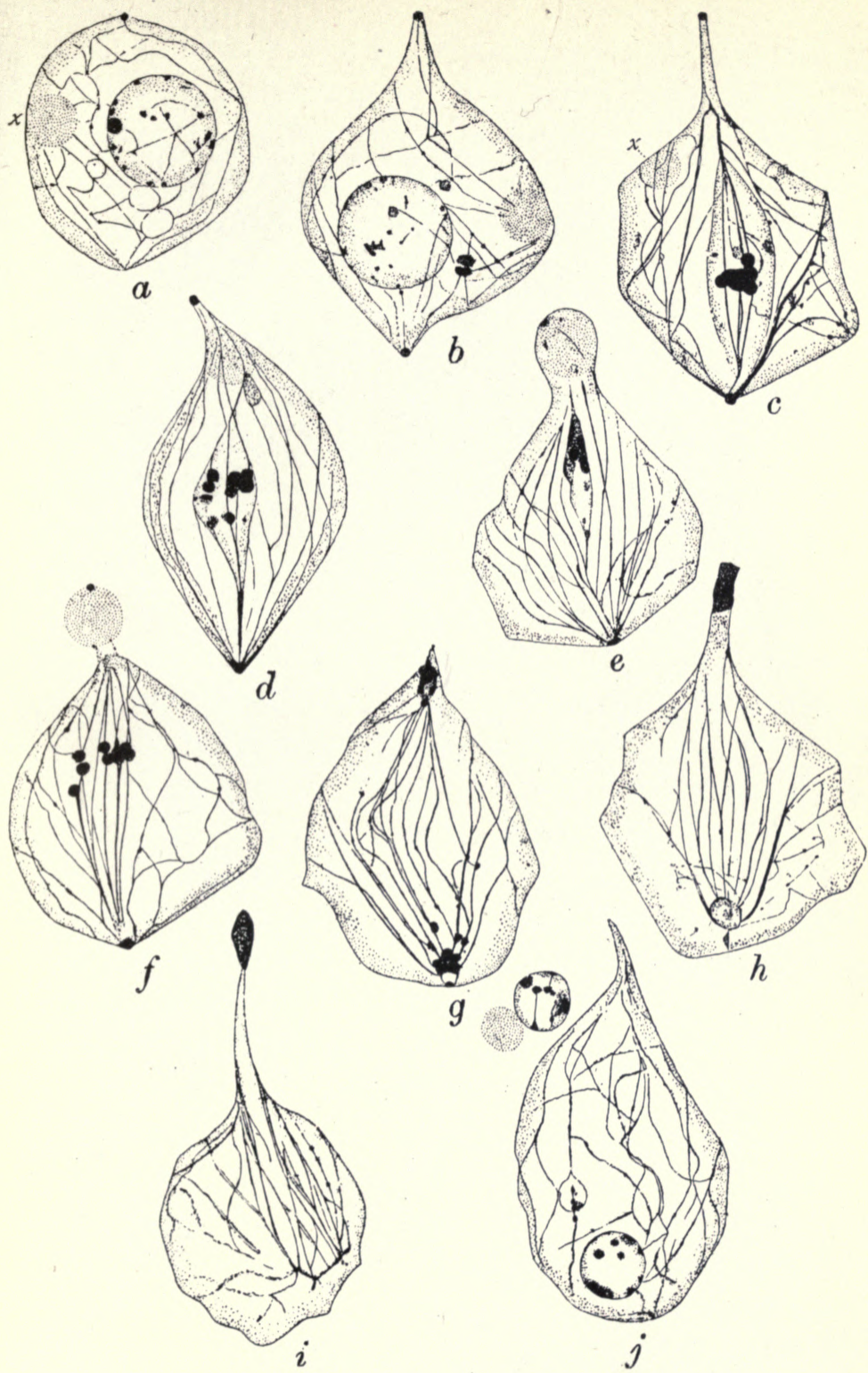

Spermatogenesis of the Hive Bee. From Mark and Copeland.

$a-e$. First (abortive) spermatocyte division, in which only a portion of protoplasm is separated. $f-j$. Second division, in which nuclear division takes place but cell division is extremely unequal. 

genesis. There are, however, a certain number of species in which females arise from virgin eggs; such species are called thelytokous. In the Hymenoptera they occur here and there among the Sawflies (Tenthredinidae) and Gall-flies (Cynipidae). In some of them no males are known and the species appear to be permanently parthenogenetic, as for example in certain species of the genus Poecilosoma among the Sawflies and in some species of Gall-flies in which the usual sexual generation seems to have dropped out, as in Cynips kollari and some species of Andricus. Other thelytokous species have occasional males, though the females are much more numerous, and in these it seems probable that both sexes may be produced from parthenogenetic eggs. The maturation of the eggs has been examined in Poecilosoma luteolum (a Sawfly) ${ }^{\mathbf{1}}$ and in Rhodites rosae ${ }^{\mathbf{2}}$, which produces the common "bedeguar" galls on the wild rose. In each case it was concluded that both the polar divisions are equational, and that no reduction in chromosome number takes place-a conclusion which seems probable a priori, since it is difficult to understand how perpetual parthenogenesis can take place with chromosome reduction in each generation, but one which requires fuller investigation before it can be regarded as definitely proved.

In the Phasmids (Stick Insects) it is known that virgin eggs frequently, and probably usually, give rise to females, though males are known sometimes to be produced from them. They also have two polar divisions of the egg, giving rise to three polar nuclei and an egg nucleus, but the details of the process are insufficiently known. Such observations as exist suggest that normal reduction occurs, but no explanation is offered of the means by which the

\footnotetext{
1 Doncaster (1906).

2 SCHLEIP (I 909).
} 
full chromosome number is restored, and the subject needs further investigation.

(4) Maturation with no polar divisions in eggs which produce females, and two in those which produce males.

This condition, which is comparable with that found in the Rotifers and referred to above, is known only in the Hymenopteran family Cynipidae (Gall-flies), in which it has been described by the writer in the common Neuroterus lenticularis ${ }^{1}$. The life-history and the cytological phenomena of the Gall-flies are so remarkable that it seems advisable to describe them in a separate section, instead of placing them with the other Hymenoptera. Typically there are two generations in the year, one of which is sexual and the other parthenogenetic; in some species, as has been mentioned previously, the sexual generation appears to have dropped out, and the flies are constantly parthenogenetic with one generation only in the year. Neuroterus lenticularis is a typical example of the species with alternation of generations. Its life-history is as follows (Pl.XVI). In May males and females hatch from the round sappy galls on the leaves and catkins of the oak. They pair, and the females lay their eggs in the tissue of the oak leaves, where they give rise to hard lenticular galls quite different from those of the previous generation. The flies which hatch in the following March or April from these galls are all females; they differ conspicuously in structure from their mothers, and lay in the oak buds eggs which develop into either males or females. The offspring of any one parthenogenetic female are all of one sex; some females produce only males, others only females, and it is further almost certain that all the parthenogenetic daughters of any one sexual female are of the same type-that is to say, any sexual female will have

1 Doncaster (1910, 1911, 1916). 


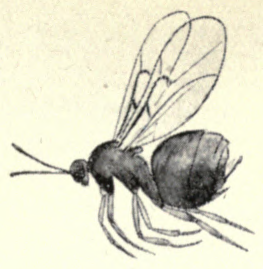

(I)

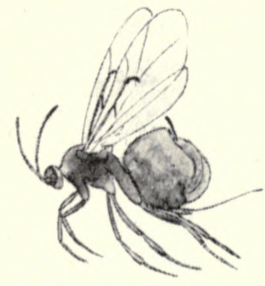

(2)

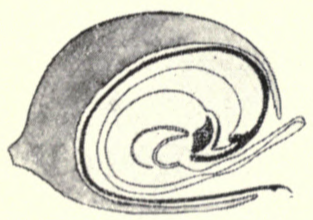

(3)

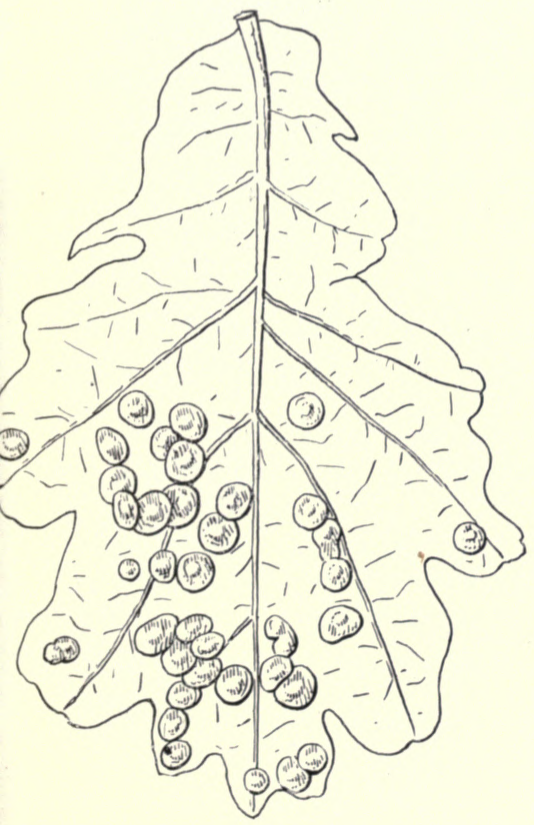

(4)

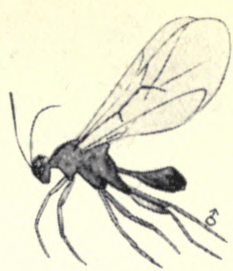

(5)

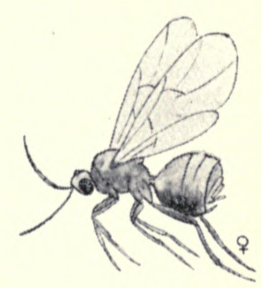

(6)

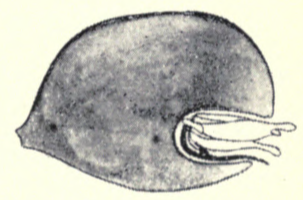

(7)

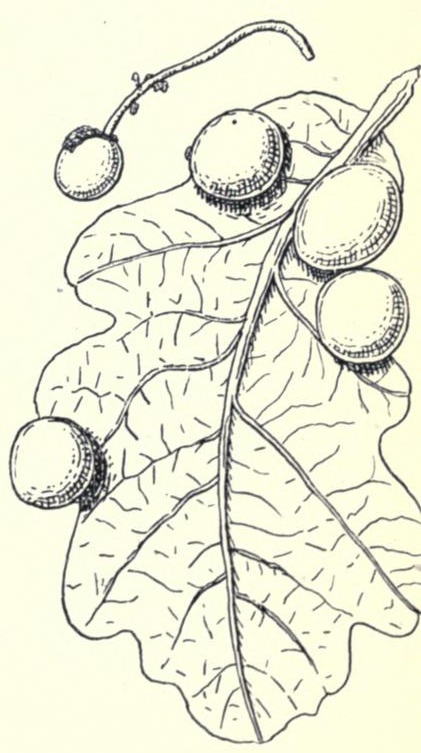

(8) 


\section{Explanation of Plate XVI.}

Life-history of the Gall-fly Neuroterus lenticularis. On the left, the parthenogenetic (spring) generation. ( 1 ) Fly with ovipositor withdrawn; (2) with ovipositor extended; (3) abdomen and ovipositor enlarged; (4) galls from which the parthenogenetic females hatch. On the right, the sexual (summer) generation, known as Spathegaster baccarum. (5) Male ; (6) female; (7) abdomen and ovipositor of sexual female enlarged; (8) galls from which the sexual generation hatch. (The figures of the flies are from drawings by F. BALFour Browne, Esq.; the galls from specimens in the Cambridge Museum.) (From the Author's Determination of Sex.) 
only male-producing or only female-producing parthenogenetic daughters.

The cytological behaviour of the eggs is not less remarkable. The fertilised eggs of the sexual generation undergo a double polar division of a peculiar type, leaving the reduced (haploid) number of 10 chromosomes in the egg nucleus. The peculiarity of the polar divisions lies in the fact that the nucleus divides in the first division without the formation of an equatorial plate; as the nuclear membrane disappears the chromosomes seem rather to sort themselves out at opposite poles of the nucleus. The egg nucleus with Io chromosomes then conjugates with the sperm nucleus also containing 10, giving rise to a zygote with 20 . This develops in to the female of the parthenogenetic generation. The eggs of different females of this generation behave differently from one another. Those of a male-producing female undergo a double polar division of the same peculiar type as is found in the sexual eggs, the chromosomes are reduced from 20 to 10 , and the egg develops with the reduced number into a male, as in other Hymenoptera. In the femaleproducing eggs the nucleus comes to the surface as if about to divide in the usual manner, but then sinks back into the egg and begins immediately to undergo the segmentation divisions. These eggs, having undergone no maturation division, contain the diploid number of chromosomes, and develop into the sexual females. No cytological explanation has yet been found of the fact that some sexual females have only male-producing daughters, others only female-producing.

(5) Parthenogenesis consequent on the entrance of a spermatozoon-" false fertilisation."

It has been found experimentally in several cases that if a spermatozoon of one species enters the egg of a species 
widely separated from it in classification, the egg may be caused to develop although the sperm nucleus, or its chromosomes, take no part in development. This has been observed, for example, in the eggs of Echinoids fertilised by sperm of the Mussel (Mytilus) ${ }^{1}$, and cytologically the development must be regarded as parthenogenetic, although a spermatozoon is present and has stimulated the egg to develop. It has more recently been discovered that a similar process takes place naturally in at least one Nematode worm-Rhabditis aberrans, described by Eva KRÜGER (1913). In this species the vast majority of specimens are protandrous hermaphrodites, which produce spermatozoa early in the stage of sexual maturity, after which only eggs are produced. Very rarely true males occur. When the eggs are ripe, spermatozoa stored up from the earlier spermproducing stage enter them, but take no part in development, and very soon degenerate. Only one polar body is formed, and in the polar mitosis the chromosomes appear in the somatic number and divide equationally. In a single egg the formation of a second polar body was observed, and in this case the egg nucleus conjugated with the sperm nucleus. It is suggested that the exceptional males are produced from these rare fertilised eggs.

The chief cytological facts of natural parthenogenesis may be summarised as follows:

I. Perhaps the most frequent type is that in which the egg nucleus undergoes one maturation division and no chromosome reduction, so that the single polar nucleus and the egg nucleus both contain the diploid number of chromosomes. This is characteristic of Crustacea, Homoptera, Cecidomyidae, and some Rotifers.

2. In rare cases (e.g. Artemia, Xenos) there may be two 1 KupelWieser (1909, I 9 1 2 ) 
polar divisions and the second polar nucleus sinks back and conjugates with the egg nucleus.

3 a. In many Hymenoptera and in the male-producing eggs of Rotifers there are two polar divisions, with reduction of the chromosomes to the haploid number. These eggs when they develop unfertilised give rise to males, which have in their primitive germ-cells the reduced number of chromosomes, and consequently undergo no chromosome reduction in spermatogenesis, the first spermatocyte division being suppressed. Eggs of this type are frequently capable of fertilisation, and when fertilised yield females.

3 b. In a few Hymenoptera and possibly some other insects there are two polar divisions both of which are equational, leaving the egg nucleus with the diploid çhromosome number. These eggs usually produce females.

4. In many Cynipidae a sexual generation alternates with a parthenogenetic. The parthenogenetic females which produce males lay eggs which behave as in $3 a$ above; the eggs of those which produce females have no maturation division.

5. In several cases under experimental conditions, and in one instance in nature (Rbabditis aberrans), parthenogenetic development of an egg is induced by the entrance of a spermatozoon, which, however, takes no direct part in development. 


\section{CHAPTER X}

\section{Artificial Parthenogenesis}

]$^{\mathrm{N}}$ addition to the naturally occurring parthenogenesis which, as has been seen in the preceding chapter, is widespread in the animal kingdom, there is another type of parthenogenesis that has attracted much attention in recent years. It has long been known that certain animals, for example the common silk-worm, occasionally lay eggs which may develop without fertilisation although they are not normally parthenogenetic, and that this occurs more readily if the eggs have been shaken or treated with acid. The matter attracted little attention, however, until J. LoeB undertook experiments with the eggs of Sea-urchins, and found that by various methods of treatment he could cause them to develop without fertilisation. It is not proposed here to enter upon a full history of the earlier experiments and the hypotheses that were put forward to account for their results, nor to consider in detail the very complicated physico-chemical problems raised, but rather to give a general account of the results obtained and their bearing on cytological study.

It has been found possible to cause parthenogenetic development of Sea-urchin eggs by very various methods of treatment. One of the earliest was by the addition of various mineral salts, such as magnesium chloride, to the sea-water, but since many salts were effective it was not possible to ascribe the initiation of development to the chemical action of specific substances; it seemed rather to be caused by the 
raising of the osmotic pressure of the water. One method, therefore, bringing about artificial parthenogenesis is by the action of hypertonic solutions. It was then found that acids and alkalis were also effective; in some cases causing only the beginning of development, which was often irregular, but in others bringing about normal segmentation followed by development into typical larvae. In the earlier experiments segmentation took place without the formation of a "fertilisation membrane" and the larvae produced were weakly and failed to develop to the later stages. Further experiment, however, proved that it was possible to induce the formation of a typical fertilisation membrane, and that by suitable treatment all the eggs could be caused to develop into quite strong healthy larvae. LoEB's improved method for bringing about artificial parthenogenesis of Sea-urchin eggs is to treat them first with butyric acid ( 2.8 c.c. $\mathrm{N} /$ Io butyric acid to 50 c.c. sea-water). Other acids, especially organic acids of the fatty series, are effective but butyric is found to be most suitable. After treatment for a few minutes (at $15^{\circ} \mathrm{C}$.) with butyric acid the eggs are transferred to pure sea-water, and then after 15 to 30 minutes to a hypertonic solution consisting of 50 c.c. seawater to which 8 c.c. $2 \frac{1}{2} \mathrm{~m}$. sodium chloride has been added. After half-an-hour in the hypertonic sea-water batches of eggs are transferred during the next half hour at five minute intervals to pure sea-water, when the eggs that have been treated for the correct time begin to segment normally. Different species of urchin, and the same eggs under different conditions of temperature or other circumstances, require somewhat different lengths of exposure to the solutions. The treatment with hypertonic sea-water may precede that with butyric acid instead of following it, but in this case the exposure must be about twice as long. 
If the solutions are used in the way described, no normal development takes place unless both solutions are used; under certain circumstances, however, it is possible to obtain development by treatment with either acid or hypotonic solution alone, although with the concentrations and length of exposure mentioned both are necessary. This fact naturally leads to the question-What are the effects of the two solutions, and how do they co-operate to bring about development? In the earlier experiments on artificial parthenogenesis no fertilisation membrane was formed, but experiments designed to elucidate the nature of this membrane have led the way to an analysis of the factors which initiate development. When the eggs have been treated with butyric acid and returned to sea-water they immediately begin to form a fertilisation membrane, but if they are left in sea-water after the membrane is formed, although a mitotic spindle may be produced, followed by division of the nucleus, they then begin to disintegrate. Droplets of protoplasm begin to be exuded, especially in the region in which the first segmentation furrow is forming, and gradually the protoplasm breaks up into fragments, the disintegration beginning at the surface and gradually extending through the egg. At a lower temperature the disintegration or cytolysis proceeds more slowly, and the egg may divide into two or more blastomeres before cytolysis has progressed so far as to destroy it. It appears, therefore, that the same treatment which brings about membrane formation also leads to cytolysis, and this conclusion has been confirmed by numerous observations on the effects of various substances on the egg. All these experiments point to the conclusion that cytolytic agents of any kind, if used in the proper concentration, tend to bring about the production of a fertilisation membrane, and to initiate cell-division, 
but unless some corrective process is applied, the cytolytic action continues and leads to the final disintegration of the egg. In the improved method for bringing about artificial parthenogenesis the function of the hypertonic solution is to have this corrective effect; its exact mode of action is unknown, but it seems to induce the formation within the egg of some substance which counteracts the later cytolytic effect of the butyric or other acid.

The cytolytic effect of the agents which induce artificial parthenogenesis can also be counteracted by depriving the eggs of oxygen after treatment with the butyric acid or other agent. Absence of oxygen completely inhibits celldivision, but if the eggs are placed in normal sea-water after being deprived of oxygen for some hours, they begin to segment in the typical manner instead of undergoing cytolysis. This might suggest that the effect of the hypertonic solution is to prevent the absorption of oxygen, but that this is not a full explanation is shown by the fact that the hypertonic solution must contain oxygen if it is to be successful. The actual formation of the membrane can take place in the absence of oxygen, but it seems that a result of the processes which lead to its production is greatly to increase the oxygen absorption of the egg, and this leads to cytolysis. If the egg is kept without oxygen for some hours, or treated with a hypertonic solution, other processes take place in the egg which regulate the oxygen absorption when the egg is replaced in sea-water, and allow normal development to occur. When development is brought about by treatment with a hypertonic solution alone, it must be supposed that this acts in a double capacity; it tends to cause cytolysis and thus to initiate development, but at the same time it corrects the excessive cytolytic effect, and allows development to proceed. 
To sum up, then, it may be concluded that the formation of the fertilisation membrane is due to a cytolytic action on the surface layer of the egg, and that a further effect of this action is to induce nuclear and cell-division, but that in many cases when once this cytolytic action has begun, it tends to continue, probably through want of regulation of the oxygen absorption, and to lead to complete disintegration. If, however, the excessive oxidation can be inhibited until the egg has restored its power of regulation, the celldivision that has been started will continue, and the egg will develop into a normal larva, which, as experiment has shown, may undergo metamorphosis and grow into a fully developed urchin.

This conclusion suggests a number of interesting enquiries, especially with regard to the nature of fertilisation by the spermatozoon and of the stimuli which lead to celldivision. To pursue these at all fully would lead into somewhat abstruse questions with regard to the physico-chemical condition of protoplasm, its permeability to electrolytes, and the nature of the chemical processes which take place in its metabolism. Work on these subjects is as yet only in its infancy, and it promises to lead to some of the most fundamental results which biological enquiry can ever hope to reach. In the present short sketch of the subject, however, only some of the more superficial aspects of the problem can be touched.

LOEB comes to the conclusion that the development of the egg when fertilised by a spermatozoon is due to the introduction of a cytolytic agent by the sperm, which alters the stability of the egg surface, so causing the production of a fertilisation membrane, and that destructive cytolysis is prevented by the presence of a second substance which inhibits any further cytolytic action. This idea is 
supported by the experiment of fertilising Sea-urchin eggs with starfish sperm (which can be done if the eggs are first suitably treated); under these circumstances some of the eggs form membranes but then disintegrate unless they are subsequently treated with hypertonic sea-water, while others continue to segment normally without the hypertonic solution. This suggests that the spermatozoon introduces a cytolytic agent into all the eggs, since all produce membranes, but that the foreign sperm does not always introduce the proper corrective to prevent complete cytolysis. Although the spermatozoon appears to introduce a cytolytic agent, this does not explain why nuclear and cell-division are brought about by its entrance, and LoEB concludes that this is caused by increased oxidation. It is known that eggs immediately after fertilisation, or after treatment which gives rise to artificial parthenogenesis, absorb oxygen much more rapidly than unfertilised eggs, and the same is true of eggs undergoing complete cytolysis; it seems probable, therefore, that the cytolysis of the egg-surface which results in membrane formation allows increased oxidation of the egg, and that this increased absorption of oxygen is the stimulus which brings about division of the nucleus followed by that of the cell as a whole.

The work of GRAY (1916) on the electrical conductivity of Echinoderm eggs before and after fertilisation or treatment with substances that bring about artificial parthenogenesis suggests a somewhat different explanation of fertilisation from that arrived at by LовB, though it does not alter the general conclusions. Gray's experiments indicate that both fertilisation and treatment with parthenogenetic agents alter the electrical condition of the egg surface, making it more permeable to electrolytes. He therefore regards the subsequent changes as due to an alteration of the surface

D. c. 
of the egg, rather than to the introduction of any substance into its interior, but there seems to be no reason against accepting this conclusion and at the same time regarding the initiation of development as depending on factors which, if unrestrained, lead on to the cytolysis of the egg as a whole.

It should also be noted that DELAGE (1908) has brought about artificial parthenogenesis by a method based on different reasoning. He considers that the rhythm of celldivision is based on a rhythmic cycle of alternate coagulation and liquefaction of the protoplasmic colloids, the formation of the centrosome, spindle and asters being due to coagulation, while the dissolution of the nucleus and splitting of the chromosomes are examples of liquefaction. He therefore subjected eggs to the successive influence of an acid and an alkali, since acids coagulate and alkalis liquefy colloids. By these means he produced very successful parthenogenetic development in Sea-urchin eggs, a result which seems to confirm his theoretical interpretation.

The changes undergone by the nucleus and cytoplasm of artificially parthenogenetic Echinoid eggs are closely similar to those seen in eggs fertilised by a spermatozoon. Slight preliminary changes occur in the interval between the treatment with butyric acid and the hypertonic solution; these are suspended while the eggs are immersed in the hypertonic solution, but as soon as they are replaced in sea-water the nucleus begins to enter the prophase stage of mitosis, and shortly afterwards an aster appears at one pole of the nucleus, the rays of which radiate from a diffuse centrosome close to the nuclear membrane. The centrosome then divides, its halves diverge and a normal spindle is formed between them. The chromosomes have by this time made their appearance in the nucleus, the membrane disappears, and the chromosomes take up their position in 
an equatorial plate on the spindle, split in the typical way, and the halves travel to the poles. The chromosome number in the first and subsequent divisions is the reduced or haploid number; although this was at one time denied, later work has proved it beyond doubt, so that here, as in the males of the Hymenoptera, an individual which appears to be normal in every respect may exist with only half the typical number of chromosomes. With regard to the origin of the centrosome and aster there has been some dispute, and the matter can hardly be regarded as finally settled. WiLson found that small asters with a central dot could be produced in non-nucleated fragments of eggs by the agents which induce artificial parthenogenesis, and both he and many other investigators believe that the centrosome arises de novo. Others, however, maintain that a centrosome persists from the polar divisions and accompanies the eggnucleus, and that it gives rise to the centrosome of the first segmentation spindle in the parthenogenetic egg ${ }^{1}$.

Although the greater part of the work on artificial parthenogenesis has been done on Sea-urchin eggs, it has been shown that the eggs of quite a large number of animals are capable of being caused to develop in this way. The methods used, of course, vary somewhat with different species, but the essentials of the process appear to be the same in all cases. The eggs of certain Starfish can be caused to develop parthenogenetically even more easily than those of Sea-urchins. Delage found that a strong solution of carbon dioxide in sea-water was an efficient means, and it has been shown that simple shaking is sufficient in some species to induce membrane formation and development. Some species may be naturally parthenogenetic, and LoEB suggests that the egg-surface is in an unstable condition so

1 E.g. Petrunkewitsch (1904), Buchner (1911). 
that a very small stimulus is required to induce the slight cytolysis which acts as the stimulus to development. The Starfish egg, unlike that of the Sea-urchin, undergoes the maturation divisions after it is shed into the sea-water, and as has been mentioned earlier, Buchner (I9II) finds that if the eggs are treated with carbon dioxide during the maturation process, after the eggs are replaced in sea-water the second polar division occurs, but the second polar nucleus sinks back into the egg and fuses with the egg-nucleus, so taking the place of a spermatozoon.

Quite a number of Annelids have been successfully caused to develop parthenogenetically, and several of them are interesting from the fact that in nature the entrance of a spermatozoon is necessary to bring about the maturation divisions. In these it is found that treatment with the parthenogenetic agent not only causes the production of a fertilisation membrane, but that this is followed by the extrusion of the polar bodies and the subsequent segmentation of the egg and development into a larva. Two curious facts with regard to Annelids may be noted. In Chaetopterus it was found that the ordinary parthenogenetic agents induced development, which went as far as a larval stage, but that no true segmentation of the egg into distinct blastomeres took place; segmentation, however, could be induced by blood serum. And in Thalassema it was observed that the polar bodies, instead of degenerating, divided mitotically several times, so giving rise to groups of cells like miniature embryos.

The eggs of several Molluscs have been caused to develop at least as far as early larval stages by methods of the same general type as are used for Echinoderms and Annelids, but in general only a small proportion of the eggs develop, no fertilisation membrane is produced, and true segmentation 
into blastomeres is more or less suppressed. In the eggs of Mactra Kostanecki observed a process of intranuclear division of the chromosomes in the first division of the parthenogenetic egg, by which he supposes that the normal somatic number may be restored, instead of the reduced number present in the egg-nucleus after maturation.

Finally, it has been found possible to cause the eggs of Frogs to develop parthenogenetically, although the methods used for Invertebrates are not effective for either Fishes or Amphibia. The first successful experiment was by GuYER, who injected frog's blood into the eggs. Bataillon then succeeded in producing parthenogenesis by merely pricking the eggs which were smeared with blood, and LoEB and BANCROFT have shown that the presence of blood is not absolutely essential. Bataillon believed that the development was caused by introduced fragments of leucocytes, which act as "catalysers," but an ingenious suggestion has been made by HERLANT with regard to the function of the blood, the introduction of which certainly facilitates development. He finds that around each leucocyte or fragment a small aster is produced, and the presence of these asters reduces the volume of protoplasm over which the main division spindle must take control before actual cell-division can be initiated. Herlant, in fact, maintains that all methods of inducing artificial parthenogenesis are either such as to increase the size of the division spindle, or to reduce the effective volume of protoplasm which it must control, and so make possible a beginning of segmentation which would otherwise fail to be effective. In the experiments of LOEB and BANCROFT, in which development was induced by simple pricking without any introduction of foreign cells, parthenogenesis is probably caused by an alteration of the surface due to the prick, comparable with 
the effect produced by shaking Starfish eggs. Larvae obtained in this way have been reared through the metamorphosis to almost or quite adult frogs, which have been found to be both males and females (LOEB, I9I8).

Although, as has been mentioned earlier, the plane of the first segmentation division in fertilised frog's eggs usually depends on the position of the entrance path of the spermatozoon, it is found that in the parthenogenetic eggs the position of the puncture has no relation to that of the first segmentation plane; this confirms the conclusion that the position of the segmentation plane depends on that of the dividing centrosome introduced by the spermatozoon, and not upon any alteration of the egg-substance induced by the spermatozoon's passage.

From this short summary of the subject it will be seen that the study of artificial parthenogenesis has led, and is likely to lead still further in the future, to important advances in our knowledge of the physiological or functional aspects of cytology. Not only is it the means of throwing important light on the nature of the metabolic processes concerned in the development of the egg, and of the physical and chemical conditions of cell-division, but it also gives us definite indications of the manner of action of the spermatozoon in bringing about development. Since eggs which under normal circumstances require fertilisation can develop to larvae or even adults without it, it is clear that the nuclear fusion which is usually regarded as the essential feature of fertilisation is not in itself the cause of development. The spermatozoon, in fact, appears to play two quite distinct parts; as the bearer of hereditary characters it is equivalent to, and of equal importance with, the egg, but as far as the early stages of development are concerned, it plays quite a minor part, and merely acts as a stimulus to a development 
which under suitable conditions can take place equally well without it. It is possible, however, to some extent to reverse the parts, for a spermatozoon may enter a nonnucleated portion of an egg and give rise to a nucleus which divides and causes the piece of egg to segment and develop into a larva. In such a case there is something of the nature of male parthenogenesis, for although the cytoplasm is of maternalorigin, the whole of the nuclear matter is descended from the spermatozoon only.

A number of problems arising from artificial parthenogenesis are still unsolved. One of the most interesting is concerned with the determination of sex, which will be further dealt with in the next chapter. If, as now seems to be almost certain, the sex of every individual depends on the presence and activity of certain chromosomes, important experimental evidence should be obtainable from animals reared to maturity from unfertilised eggs, but unfortunately few animals have yet been reared in this way to a stage at which the sex could be decided with certainty. Lов B (I9I8), however, finds that parthenogenetically-produced frogs may be either male or female; out of nine individuals reared to an age at which the sex could be distinguished with certainty seven were male and two female. It appeared that the males had the diploid chromosome number in the spermatogonial nuclei. 


\section{CHAPTER XI}

\section{The Cytological Basis of Sex-Determination}

]$^{\mathrm{N}}$ the chapter (Chapter IX) on natural parthenogenesis reference was made to several examples of eggs, the development from which of males or females is dependent either on the nature of the maturation process, or on whether the egg is or is not fertilised. Such cases naturally lead to the suspicion that the determination of sex, that is, the production of a male or a female, is dependent on the cytological condition of the egg before development begins, and a great amount of evidence pointing toward the same conclusion has been accumulated in recent years. This is not the place to discuss the question whether sex is in all animals irrevocably determined from the beginning of development ${ }^{1}$; all that can be done is to describe the evidence which shows that it is so in many cases.

Perhaps the clearest instances of determination of sex by a cytological difference in the egg before development begins are provided by the Hymenoptera. In every example of this order of insects which has been examined, the female is derived from an egg containing the diploid number of chromosomes and the male from one with only the haploid number. Most frequently the difference depends on whether the egg is fertilised or not, as was first discovered in the Honey Bee, and subsequently shown to be true of a number of other species. In all these cases fertilised eggs yield females and unfertilised eggs males, and it is remarkable that

1 For a discussion of this problem reference may be made to the writer's book The Determination of Sex (Camb. Univ. Press, 19 I 5 ). 
in the social species, such as bees and wasps, subsequent treatment of the female larva may turn it into a worker or a queen, but never into a male, nor a male larva into a worker. In the Gall-flies (Cynipidae) the difference between the eggs with the haploid and diploid chromosome numbers is produced in a different way, for both males and "sexual"
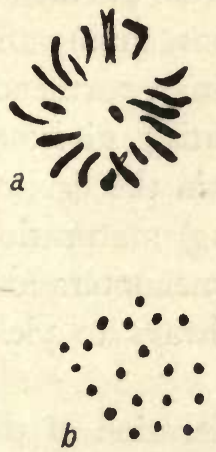
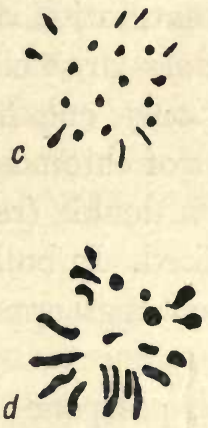
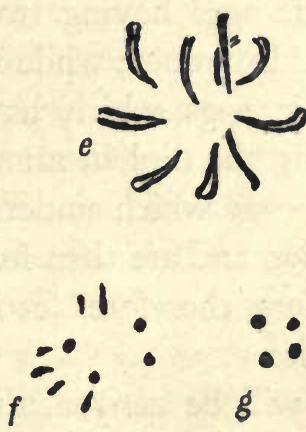

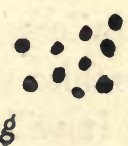

Fig. 17. Chromosome-groups of dividing cells in the Gall-fly Neuroterus lenticularis. (Preparations by the writer.)

a. Diploid equatorial plate in ovary of sexual female.

b. Diploid anaphase group in ovary of sexual female.

c. Diploid anaphase group in nervous system of female larva (cf. $e$ ).

d. Diploid equatorial plate of segmentation divisions in egg of spring (agamic) generation, which would develop into a female (cf. $g$ ).

e. Haploid metaphase group in nervous system of male larva; each chromosome split (cf. c).

$f$. Haploid anaphase group in spermatogonial division.

g. Haploid anaphase group in segmentation division in egg of spring (agamic) generation, which would develop into a male (cf. $d$ ).

females arise from parthenogenetic eggs. These eggs are of two kinds, laid by two classes of females; those of one kind undergo a double maturation division, leaving the haploid number of chromosomes in the egg, and grow up into males, while those of the other kind have no maturation division and have thus the diploid number and develop into females. 
The first type of sex-determination by means of a difference of the chromosomes in the egg at the beginning of development is thus that in which a male-determining egg has $n$ chromosomes and the female-determining egg of the same species $2 n$. This type is best known in the Hymenoptera. It probably occurs also in the Rotifers, in which it seems fairly clear that males are produced from parthenogenetic eggs having two maturation divisions, giving the haploid number; while females arise either from parthenogenetic eggs which have only one maturation division, leaving the diploid number of chromosomes in the egg, or from eggs which undergo a double (reducing) maturation division and are then fertilised. In both Hymenoptera and Rotifers, therefore, fertilised eggs appear always to yield females.

It will be convenient to postpone consideration of the other chief group of parthenogenetic species-that including the Aphids and parthenogenetic Crustacea-in which both sexes may arise from unfertilised eggs having only one maturation division, and in which, as in the Hymenoptera and Rotifers, fertilised eggs always produce females. The phenomena presented by this group will be more easily understood after some account has been given of the relations between chromosomes and sex in animals in which the eggs all require fertilisation.

In the account of the maturation of the germ-cells, and of fertilisation, given in Chapters V-VIII, it was assumed that the chromosome-groups of the male and female, and hence of the sperm and egg, were alike. In a large number of animals this is to all appearance true, but in recent years it has been shown that in many species, belonging to very different groups, such a statement is not strictly correct. Nearly all the chromosomes do accurately correspond in the 
two sexes, so that if we letter them $A, B, C, \ldots$ both male and female will contain in each nucleus $A, A, B, B, C, C, \ldots$ But it was discovered by several investigators about the beginning of the present century that in the males of certain insects of the orders Hemiptera and Orthoptera one chromosome behaved differently from the others during the stages preceding the maturation divisions of the spermatozoa. This chromosome, at first known as the accessory chromosome, was found to take no part in the spireme and synizesis stages; while these stages are in progress it remains a compact deeply staining body which was sometimes described as a chromatin nucleolus. When the other chromosomes emerge from synizesis and contract to form the double chromosomes of the first maturation division, the "accessory" also gives rise to a definite chromosome which takes its place on the spindle with the rest. It may differ from the others in size, being either larger or smaller, but this is by no means invariable. When the bivalent chromosomes divide into their component units and pass to the poles, the accessory usually passes undivided to one pole of the spindle, and hence has been called the beterotropic or hetero-cbromosome, the name by which it is now most frequently called. Very often, however, especially by American writers, it is known simply as the $X$-chromosome. When, as most frequently happens, the heterotropic or $X$-chromosome passes undivided to one pole of the first spermatocyte division-figure, it divides equationally in the division of those secondary spermatocytes which contain it. In the Hemiptera the process is reversed; it divides longitudinally in the first spermatocyte division and then passes over undivided in the second division into half the spermatids. In either case, therefore, since this chromosome passes to one pole without division in one or other of the spermatocyte mitoses, it 
follows that half the spermatids contain it and half are without it, so that the resulting spermatozoa are of two kinds which are produced in equal numbers (P1. XVII, I-5).

After these phenomena had been observed in the spermatogenesis of several insects, a comparison was naturally made of the oogenesis of the same species, and it was then discovered that in each case the female contained two similar chromosomes corresponding with the heterotropic or $X$-chromosome of the male. These usually differ from the $X$-chromosome of the male in not forming a chromatin nucleolus in the spireme stage of the oocyte; they behave at this stage like the other chromosomes and unite to form a bivalent element, the two members of which separate in the first maturation division of the egg and divide equationally in the second, with the result that all the mature eggs contain a single $X$-chromosome.

From these facts it was natural to infer that the $X$ chromosome acts as a determiner of sex, for since all the eggs before fertilisation contain one such body, and half the spermatozoa contain it while half do not, it follows that half the fertilised eggs will contain one $X$ and the other half two, an expectation which has been verified by the examination of the mitotic figures in the segmentation of the eggs. It is known, however, that adult males of these species contain one $X$-chromosome while females contain two, and it seems natural to infer that the spermatozoa which contain an $X$-chromosome cause the eggs which they fertilise to, become females, while those which lack the $X$ are maledetermining. Some further consideration of this conclusion will be given at a later stage.

An obvious objection to this hypothesis of sex-determination was at first a difficulty. The known cases in which 

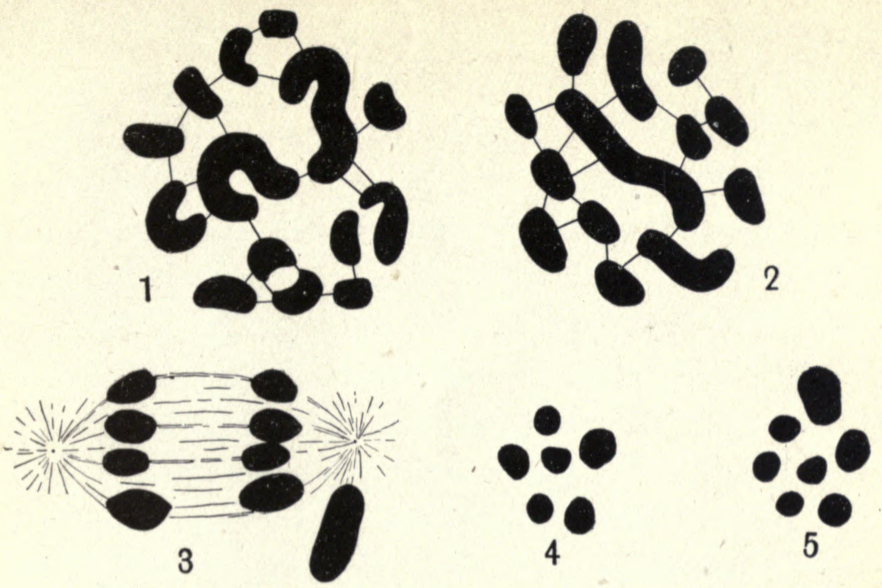

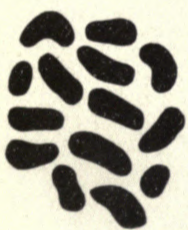

6

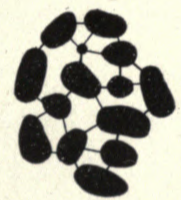

7
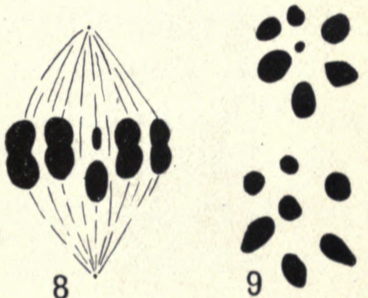

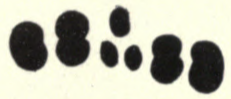

10

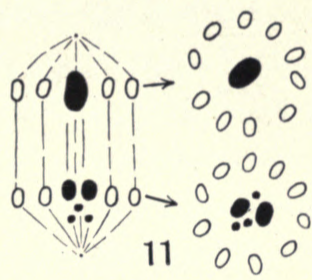

Hetero- and Idio-chromosomes in Insects (Hemiptera). (Reproduced, by permission of the Editor, from Quart. Journ. Micr. Science, LIX (I9I4), p. 499.) (I-5) Protenor belfragei. (I) Female, equatorial plate before reduction, two large hetero-chromosomes. (2) Similar group of the male, one large hetero-chromosome. (3) Spindle of second maturation-division of spermatozoon; large hetero-chromosome below right pole. $(4,5)$ Two daughter-plates of same division; hetero- chromosome in group 5. (6-9) Euschistus variolarius. (6) Female, equatorial plate before reduction; no conspicuously small chromosome. (7) Similar group of the male; one very small idio-chromosome. (8) Spindle of second maturation-division of spermatozoon ; large and small idio-chromosomes in centre. (9) Two daughter-plates of same division ; small idio-chromosome in upper, large in lower. (10) Rocconota annulicornis. Side view of equatorial plate of second maturation-division of spermatozoon, showing large idio-chromosome paired with two small. (I I) Acholla multispinosa, second maturationdivision of spermatozoon, showing large idio-chromosome paired with five small. (I-IO from WiLson; i I from Wilson, after PAYNE.) 
the male had one hetero-chromosome and the female two were few, and as far as was known the chromosome-groups of the two sexes in the great majority of animals were alike. It thus seemed difficult to suppose that in a few species sex was dependent on the presence or absence of a particular chromosome while in many others no such difference existed. This difficulty has been to a great extent removed by a discovery made almost simultaneously by Miss N. M. Stevens and E. B. Wilson (1905-6), and since extended by other investigators to a large number of different species. These American cytologists observed that in certain Beetles and Hemiptera, although both sexes possessed the same number of chromosomes, the male had an unequal pair which behaved in the spireme stages like the heterotropic chromosome, but came together as a bivalent and separated from one another in one of the spermatocyte divisions. In this division it was easy to see that the two members of the pair were of different sizes, so that two kinds of spermatozoa were formed, one having a large chromosome and the other a small one. In the female, however, as in the species in which the male possesses only a single hetero-chromosome, there is an equal pair, both members being of the same size as the larger member in the male. Chromosomes of this kind WiLson called idio-chromosomes, a term which is often used in a rather more general sense to include the heterochromosome as a special case. Frequently these chromosomes are spoken of as $X$ - and $Y$-chromosomes; it is said that the male has an $X-\Upsilon$ pair, while the corresponding female has two $X$-chromosomes (Pl. XVII, 6-9).

By investigating a number of species WILson found that he could obtain a series ranging from forms in which no $r$-chromosome was present, through those in which it was very small, and others in which it was nearly as large as the 
$X$-chromosome, to the final condition in which the two idio-chromosomes were of the same size. Or, probably more correctly, if we begin with species in which both sexes have two apparently similar idio-chromosomes, we find all stages of the degeneration of one of them in the male until we reach the stage at which the smaller or $r$-chromosome has disappeared altogether. We may therefore suppose that in these species the female has always two $X$-chromosomes while the male has an $X-r$ pair. In many forms $X$ and $r$ are not visibly different and in these the chromosome-group of the male is indistinguishable from that of the female; in others the $r$ is reduced in size, and all stages of its reduction can be found till the stage is reached at which the male has one idio-chromosome (hetero-chromosome) and the female two. In all these cases, therefore, the male may be regarded as producing in equal numbers two kinds of spermatozoa, $X$ - and $Y$-bearing, while all the eggs bear $X$, with the result that two kinds of zygotes are formed, $X Y$ and $X X$, of which the former kind will become males and the latter females. On this hypothesis, therefore, those species in which the male has only a single heterotropic chromosome constitute only a special case of a phenomenon which is of much more general occurrence-the determination of sex by spermatozoa which either do or do not contain a female-determining $X$-chromosome.

This condition in which the male contains either only a single "sex-chromosome," or an unequal pair of idiochromosomes, while the female has two equal chromosomes corresponding with the larger or " $X$ " element in the male, has now been found in a large number of animals of various groups. In addition to a number of species of Hemiptera (e.g. Protenor, Anasa (Coreidae), Pyrrbocoris, Pentatoma, Lygaeus, Euschistus, etc.) and Orthoptera (e.g. Blatta, 
Forficula, Locusta, Gryllus, Stenobotbrus, etc.), the Orders in which it was first observed, it has been found in many Insects of the Orders Diptera, Coleoptera and Neuroptera, in Myriapods, in some Spiders, in a number of Nematode worms, and in several Mammals, including Man. A somewhat similar condition has also been described in one or two Molluscs and in a Trematode (Schistosoma $=$ Bilbarzia). It is thus a phenomenon of widespread occurrence, but, as will be seen later, there is evidence that it is not by any means universally true that sex is determined by the presence or absence of a particular sex-chromosome in the spermatozoon. In the groups in which this condition is found, even when the " $X$ " and " $Y$ " chromosomes of the male are alike, they can usually be distinguished from the other chromosomes (sometimes called autosomes) by their different behaviour in the spireme stages and also not infrequently by the fact that)in the maturation mitoses they divide and pass to the poles either rather before or rather after the ordinary "autosomes." Most frequently the idiochromosomes lag behind the autosomes in the spermatocyte anaphases, and the presence of such a lagging pair has sometimes been the first observed indication of the existence of a pair of idio-chromosomes. Another difference between the idio-chromosomes and the autosomes that is characteristic of the Hemiptera, but not usually found in other groups, is that the two idio-chromosomes divide equationally in the first spermatocyte mitosis and then pair and separate to opposite poles in the second. The autosomes in this Order, as elsewhere, pair and separate in the first division and divide equationally in the second.

In some species of Insects the condition is rather more complicated than in the examples described above. Several cases have been described in which the male has a multiple 
$X$-chromosome, but only one $r$ element. In a local race of the Pentatomid species Thyanta custator, for example, WiLson found that two chromosomes both behave as mates for the single $Y$-chromosome; they pair with it in the prophase and both separate from it in the second spermatocyte mitosis, the $Y$ going to one pole and the two $X$ elements to the other. The female has four $X$ elements, so that two are present in every mature egg. In anòther Hemipteran, Acbolla, F. PAyne has described an $X$-group consisting of five chromosomes, two fairly large and three very small, all of which pair with a single $Y$-chromosome and pass together to one pole while the $r$ goes to the other (Pl. XVII, IO, I I). Another complication, with which the origin of these multiple idio-chromosomes is possibly connected, arises from the occasional presence of supernumerary chromosomes, such as have been described by Wilson in three species of the Hemipteran genus Metapodius (P1. XVIII). In these species the typical condition is that both sexes have 22 chromosomes, the male an unequal $X-\gamma$ pair, and the female two similar $X$ elements. In some races, however, the $r$-chromosome is absent in the male, giving $2 \mathrm{I}$ in the male and $\mathbf{2 2}$ in the female. In other races there may be a variable number of small "supernumerary" chromosomes, which behave exactly like idio-chromosomes in the spireme stage and in the mitotic figure. They may attach themselves to either the $X$ - or the $Y$-chromosome, and pass with it to its pole of the spindle, and the variability of their behaviour in this respect leads to the production of individuals with any number up to 28 (i.e. twenty autosomes, one pair of idio-chromosomes, and up to six supernumeraries). WiLson suggests that they arose originally by abnormal division in which both $X$ and $r$ went together to one pole ("non-disjunction," see below, p. 232), 

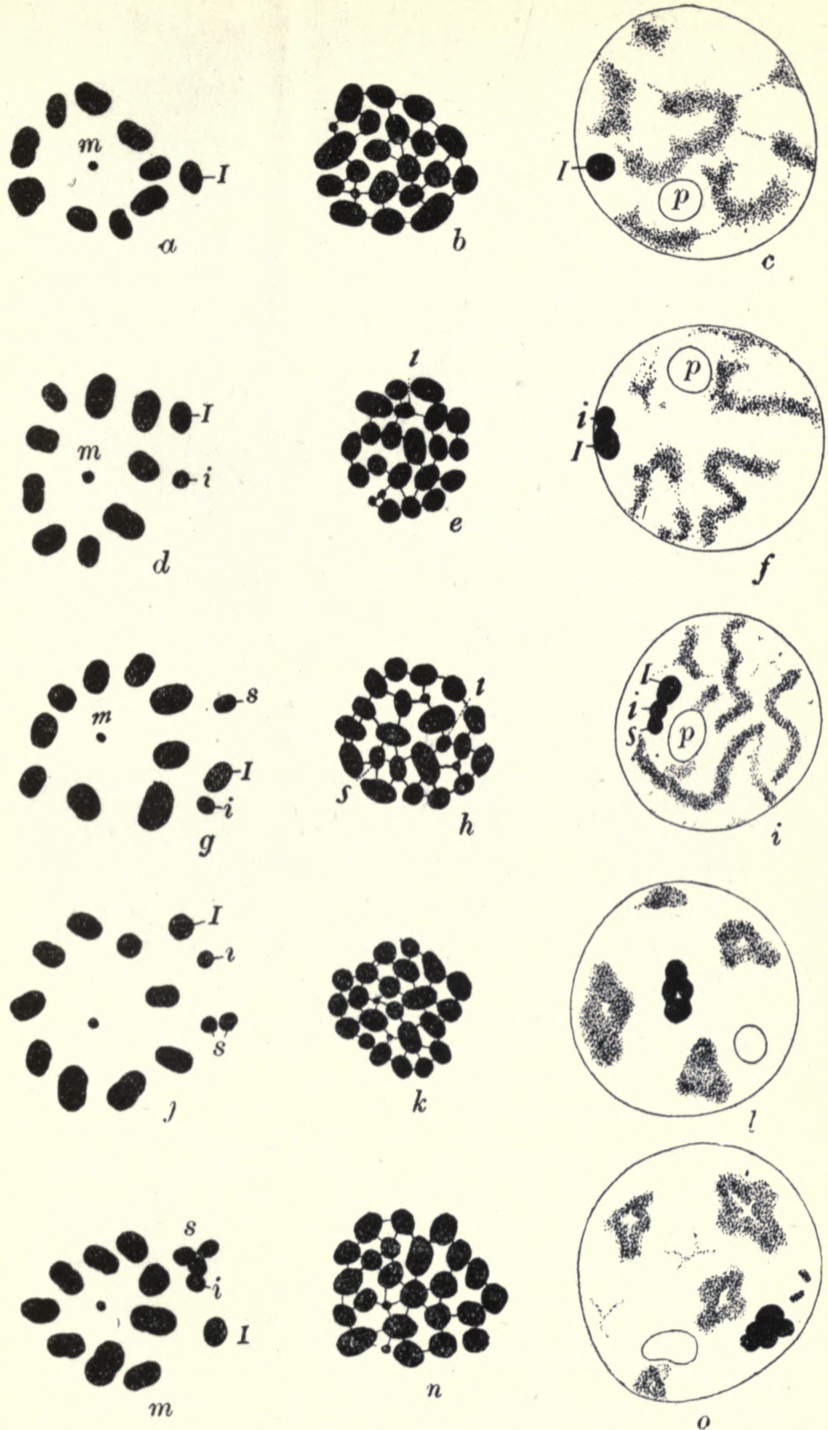

Chromosome groups of Metapodius, from Wilson ( $1909 b$ ).

$a, d, g, j, m$. First spermatocyte metaphase; $b, e, h, k, n$, spermatogonial metaphase ; $c, f, i, l, o$, nuclei in growth-period.

$a-c, 2$ I-chromosome form ; $d-f, 2$-chromosome form ; $g-i$, 23-chromosome form, one supernumerary ; $j-l, 24$-chromosome form, two supernumeraries; $m-0,25$-chromosome form, three supernumeraries.

$I, i$. Large and small idio-chromosomes ; $m, m$-chromosome ; $p$, plasmosome ; $s$, supernumerary chromosome. 

giving rise to a spermatozoon with both $X$ and $r$. This, fertilising an $X$-bearing egg, would give rise to a female with two $X$-chromosomes and a supernumerary $(\eta)$, and all the observed combinations can easily be derived from this by suitable matings.

Another point that must be noted is that the $X$-chromosome (heterotropic chromosome) may be closely associated with one of the ordinary "autosomes"; this has been observed as a constant condition in certain Orthoptera, and is

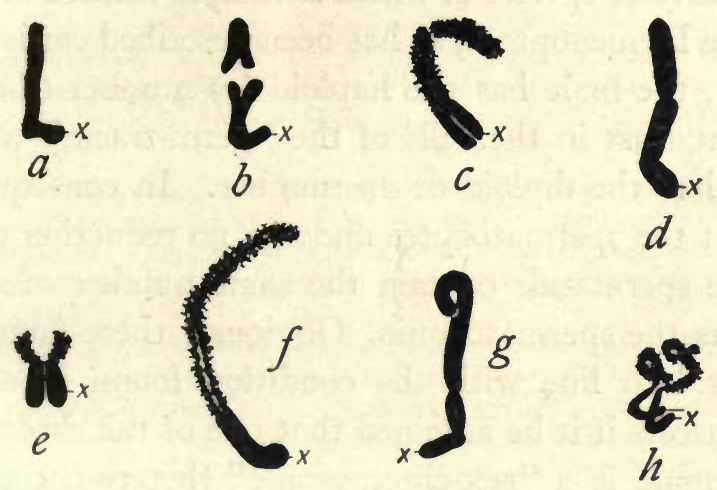

FIG. 18. Compound groups formed by union of the $X$-chromosome with other chromosomes in Orthoptera (from WiLson, 1911). $a, b$ after SinÉTY, $c$ - $h$ after McClung.

also sometimes found in the Nematode Ascaris megalocephala, though in other individuals of the same species the $X$-chromosome is quite independent of the others (Text-fig. I8).

Although, therefore, the details vary considerably, it is clear that in a large number of animals of very different groups the male differs from the female in not having one, or in some cases more than one, chromosome which is present in the female; either the chromosome is absent altogether in the male, or it is represented by an element

D. c. 
which is different from, and commonly smaller than, the corresponding body in the female. As a result the male produces two kinds of spermatozoa, one containing a chromosome-group exactly similar to that present in all the eggs, and the other differing in respect of the sex- or idio-chromosome. It remains to consider how these facts can be related, firstly to the condition already described in the Hymenoptera, secondly to the production of males and females by parthenogenesis in such forms as the Aphids, and thirdly to species in which hermaphroditism occurs.

In the Hymenoptera, as has been described earlier in this chapter, the male has the haploid ( $n$ ) number of chromosomes at least in the cells of the "germ-track," while the female has the diploid or $2 n$ number. In consequence of this fact the spermatocytes undergo no reduction division, and the spermatids contain the same number of chromosomes as the spermatogonia. Obviously these facts can be brought into line with the condition found in so many other insects if it be assumed that one of the chromosomes of the male is a "sex-chromosome" (hetero-chromosome) and that therefore two of those of the female are sexchromosomes. If this assumption is made, it is clear that the Hymenoptera are similar to the other insects described in having a single hetero-chromosome in the male and two in the female, but since there is no reduction in the spermatogenesis all spermatozoa will contain the hetero-chromosome and therefore all fertilised eggs will contain two and will become females. From the facts of artificial parthenogenesis it is known that a normal individual can develop from an egg containing only the haploid number of autosomes, and it may thus be supposed that the Hymenoptera offer only a special case of the condition found in so many other insects, being similar in respect of the sex-chromo- 


\section{Explanation of Plate XiX.}

Diagram of chromosome-cycle of Phyllaphis corveni.

At the top, chromosome group of stem-mother with four autosomes and two $X$-chromosomes. From this three lines are represented as diverging, leading on the left to a female which will produce a sexual egg, in the middle to a female which will reproduce parthenogenetically, and on the right to a male.

2nd and 3 rd lines, metaphase groups and diagrammatic anaphases of three eggs, of which the left and middle will produce females, the right a male. In the female-producing eggs the $X$-chromosomes divide equationally; in the male-producing they pair and separate, so that the male has only one (4th line).

$5^{\text {th }}$ line, on the left, metaphase group of first polar division of the sexual egg, all chromosomes paired. Below this, anaphase of first, and telophase of second polar division, leaving three chromosomes in the egg.

$5^{\text {th }}$ line, on the right, metaphase group of first spermatocyte division, with paired autosomes and single $X$-chromosome. Below this, diagrams of the first (unequal) and second (equal) spermatocyte divisions, leading to spermatozoa with three chromosomes and a small degenerate cell with two. Union of egg and spermatozoon restores the number six in the stem-mother. 

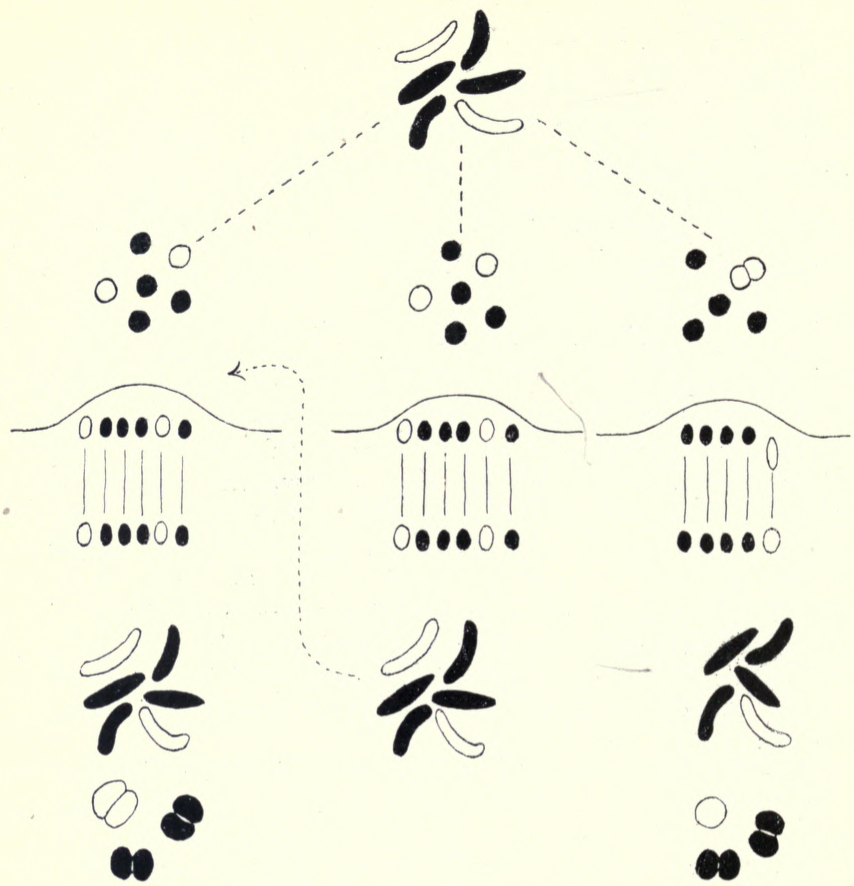

$\infty$ *

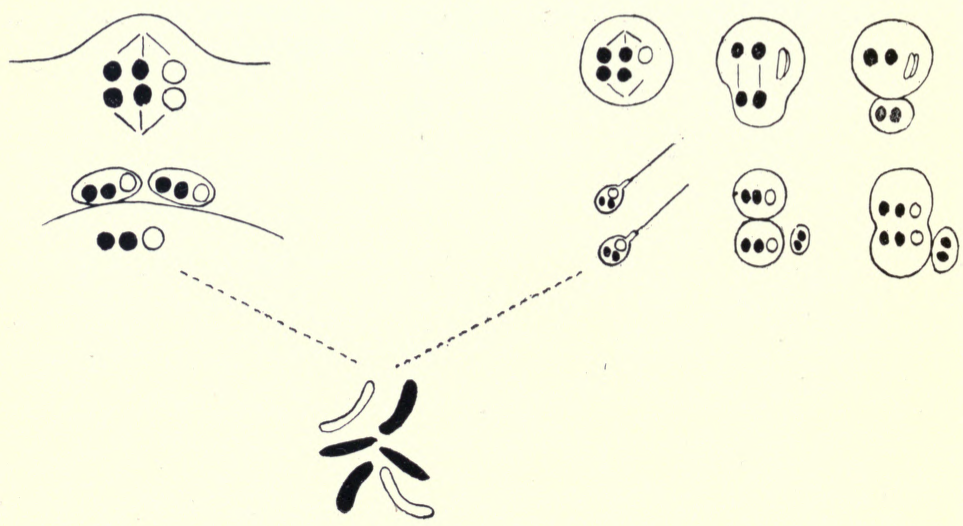


somes but differing in the existence of only the haploid number of autosomes in the male instead of the diploid number that is present in almost all other groups.

The phenomena seen in the parthenogenetic Aphids and Phylloxerans are more closely comparable with those of the sexually reproducing species, although some points of great interest have not yet been completely worked out. Our knowledge of the main facts depends chiefly on the work of Miss Stevens (1909, I910 a), T. H. Morgan (1915) and von Baenr (I909); Morgan's account of Phyllaphis coweni may be taken as typical (Pls. XIX, XIII). In this species the nuclei of the "stem-mother" (female produced from a fertilised egg) contain six chromosomes, two of which are $X$-chromosomes. She produces two kinds of eggs, (I) eggs that will develop parthenogenetically into females, and (2) eggs that develop parthenogenetically into males. The females produced from these eggs are either sexual females or again reproduce parthenogenetically like the stem-mother. There are thus three kinds of eggs in all, (I) and (2) which develop parthenogenetically and produce respectively females and males, and (3) sexual eggs which require fertilisation and produce females (stem-mothers). The oocytes of class (3) undergo a synapsis (synizesis) stage and then have a double polar division, reducing the chromosomes to three-two autosomes and one $X$-chromosome. The oocytes that will develop parthenogenetically have no synapsis, and these eggs undergo only one maturation division. In those which will produce females all the chromosomes divide equationally, so that the female offspring, like the mother, have six chromosomes. In those that produce males, however, the two $X$-chromosomes pair together before the single polar division, and while the autosomes are dividing equationally the $X$-chromosomes separate from each other, one going 
into the polar body and the other remaining in the egg. As a result, the male has five chromosomes-four autosomes and one $X$-chromosome-as compared with the six of the female. As in many other Hemiptera, therefore, the male has one $X$-chromosome while the female has two, and this 'condition is reached by the $X$-chromosomes undergoing a reduction division at the time when the autosomes are dividing equationally in the single polar mitosis of the maleproducing parthenogenetic egg.

In the Aphids, however, as in other parthenogenetic forms, only females arise from fertilised eggs, and since the male has only one $X$-chromosome one would expect that two kinds of spermatozoa would be formed, and therefore the production of nothing but females from fertilised eggs requires explanation. The explanation of this apparent anomaly is that the first spermatocyte division is an unequal one. Just as in other forms the autosomes come together in pairs in the preceding growth-phase, giving rise to three chromosomes in all, two bivalent autosomes and the single $X$-chromosome. In the first spermatocyte division, as in other Hemiptera, the bivalent autosomes divide into their separate components and the $X$-chromosome splits longitudinally, but in the anaphase of the mitosis both halves of the $X$-chromosome travel together into one daughter-cell; they lag behind the autosomes but in the telophase both halves are included in one nucleus, which thus contains two single autosomes and a divided $X$ chromosome, while the other nucleus contains only two autosomes (cf. Pl. XIII, 9-II). When the cell divides the division is unequal; the cell containing the three chromosomes is much larger than that which has only two. The first spermatocyte division thus results in a large secondary spermatocyte containing two autosomes and an $X$-chromo- 
some, and a small one containing only the two autosomes. This smaller cell never divides again; it persists for a time and then degenerates. The larger secondary spermatocytes proceed almost immediately to the second division, in which all three chromosomes divide equationally, producing two equal spermatids each with three chromosomes. The final result of the process, therefore, is that every spermatogonium gives rise to two spermatids each with an $X$-chromosome, and to a small degenerate cell which disappears. Since all the mature eggs contain two autosomes and an $X$-chromosome, all the fertilised eggs have four autosomes and two $X$-chromosomes, and thus all develop into females.

An interesting problem arises with regard to the cause of the throwing out of one $X$-chromosome in the polar body of the male-producing eggs. In Phyllaphis the same female may produce both female- and male-producing eggs, but in Pbylloxera the males and females are produced from different parents, and in some species the two kinds of eggs are of different sizes. In Phylloxera caryaecaulis investigated by Morgan the life-cycle is as follows. The "stem-mothers,"

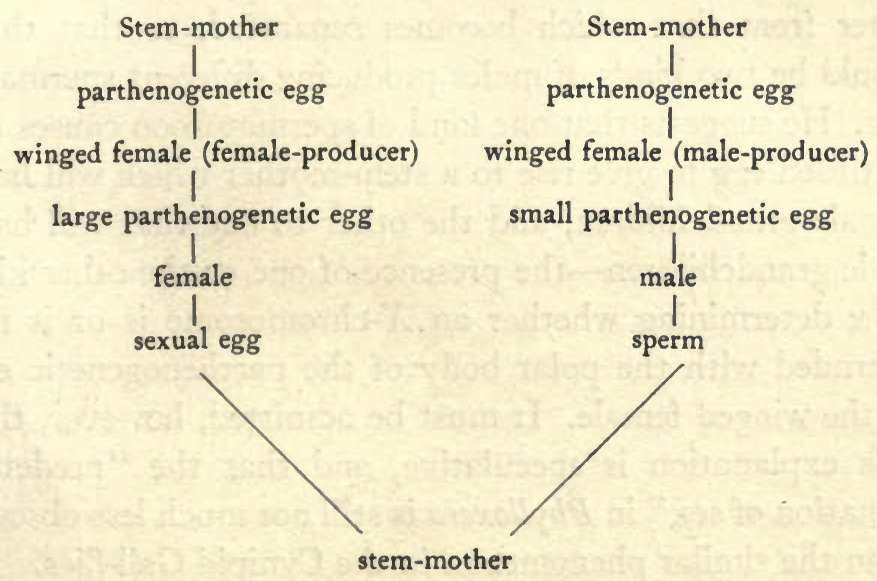


derived from fertilised eggs, produce galls on the hickoryleaf. They lay parthenogenetic eggs in the gall, and from these eggs winged females develop. These are of two kinds; some lay small eggs from which males hatch, others large eggs which produce females, and since all the individuals in one gall are of the same kind, the stem-mothers must also be of two kinds-those with male grandchildren and those with female. The females produced from the winged forms each contain one egg which requires fertilisation and gives rise to the stem-mother again. The life-cycle is represented schematically on the preceding page.

In the $P$. caryaecaulis female there are four autosomes, two large $X$-chromosomes and two small " $x$-chromosomes" which are attached to the $X$-chromosomes. In the male there are the four autosomes but only one $X$ and one $x$, the other $X x$ having been extruded with the polar body of the egg. In the spermatogenesis of some males Morgan found that the $X$ and $x$ remain attached to each other, while in others they separate, giving apparently five chromosomes in some and six in others. He suggests that the $x$ which remains attached to the $X$ is different in character from that which becomes separated, so that there would be two kinds of males producing different spermatozoa. He suggests that one kind of spermatozoon causes the fertilised egg to give rise to a stem-mother which will have female grandchildren, and the other to one that will have male grandchildren - the presence of one or the other kind of $x$ determining whether an $X$-chromosome is or is not extruded with the polar body of the parthenogenetic egg of the winged female. It must be admitted, however, that this explanation is speculative, and that the "predetermination of sex" in Phylloxera is still not much less obscure than the similar phenomenon in the Cynipid Gall-flies. 


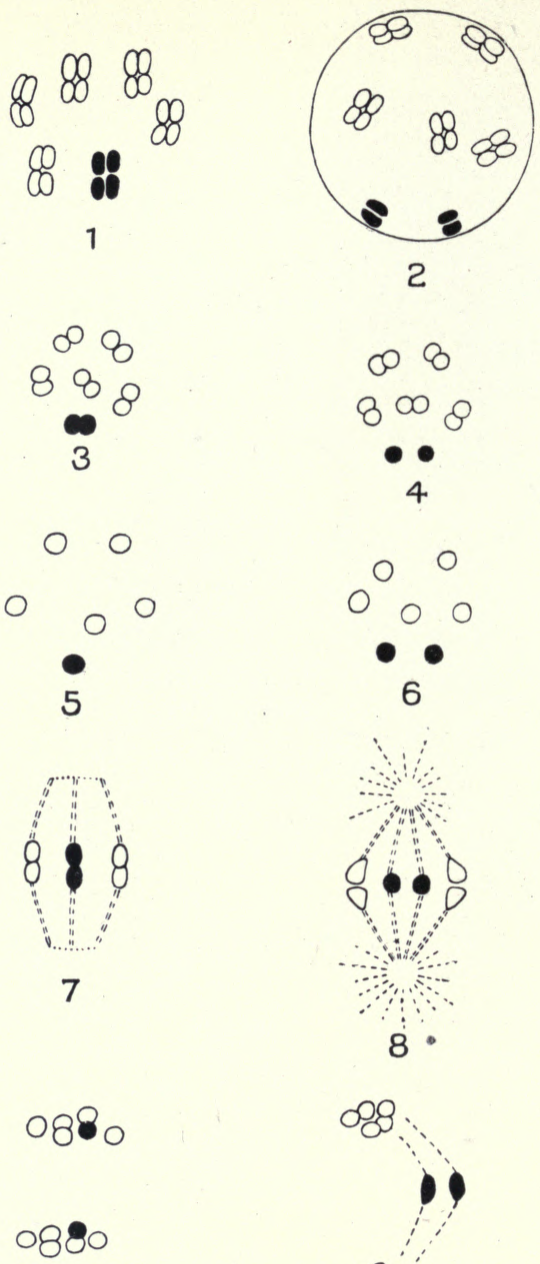

9

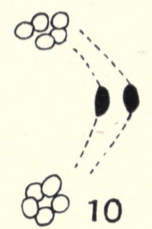

Chromosomes in the development of eggs and spermatozoa of the hermaphrodite generation of Rhabdonema nigrovenosum. (After Bover I, I 9 I I, reproduced by permission of the Editor, from Quart. Journ. Micr. Science, Lix ( I 9 I 4), p. 5 ○I.) Oogenesis on left, spermatogenesis on right. Hetero-chromosomes black. ( 1,2 ) Early stages of first maturation division of egg and spermatozoon. (3, 4) Daughter groups of first maturation division. $(5,6)$ Equatorial plates of second maturation division. $(7,8)$ Diagrammatic side views of same stage. (9, 10) Side views of last stage of second maturation division. 
Our knowledge of the behaviour of the sex-chromosomes in hermaphrodite species is still far from complete. The best-known case is that of the Nematode Rbabdonema (Rbabditis) nigrovenosum studied by BoverI (I9II) and by Schleip (I9II). This species has two generations in the complete life-cycle, one of which consists of hermaphrodites living as parasites in the lungs of the Frog, while the other is free-living and consists of males and females. The somatic chromosome number of the hermaphrodites and free-living females is twelve, and the males have eleven; that is to say, the hermaphrodites and females have ten autosomes and two $X$-chromosomes, and the males only one $X$-chromosome (Pl. XX). In the spermatogenesis of the free-living males two kinds of spermatids are produced, one of which has six and the other five chromosomes. Boveri supposes that the spermatozoa having five, and thus lacking the $X$-chromosome, are functionless, so that all fertilised eggs contain twelve, and develop into hermaphrodites. The evidence, however, for the degeneration of the spermatozoa without an $X$-chromosome is at present very slender. In the hermaphrodite generation the gonad consists of two parts, a larger ovarian portion and a smaller testicular portion which produces spermatozoa. The primitive germ-cells, whether oogonia or spermatogonia, have twelve chromosomes. In oogenesis all these chromosomes behave alike; before the polar divisions they unite in pairs to form six bivalents, and the two maturation mitoses leave six chromosomes in the egg. In the spermatogenesis of the hermaphrodites, however, the two $X$-chromosomes behave differently from the others. Instead of pairing to form a bivalent they usually remain separate; only rarely do they pair. In the first spermatocyte division the bivalent autosomes separate into their single components, while the $X$-chromosomes 
divide equationally. In the second spermatocyte division the autosomes divide equationally, but the two $X$-chromosomes pair and separate towards opposite poles. They lag behind the autosomes, and according to Schleip one is included with the daughter-nucleus at one pole, while the other lags so much that it remains outside the nucleus in the telophase and is ultimately extruded from the spermatozoon with the "Spindelrestkörper." As a consequence, half the spermatozoa have an $X$-chromosome and half are without it. Boveri describes the second spermatocyte mitosis rather differently; he believes that the two $X$-chromosomes may either go both to one pole, giving spermatids with seven and five chromosomes (two $X$ and no $X$ ), or may separate from one another and go one into each spermatid, giving six in each. He supposes that the spermatids with seven are functionless, that those with six cause the fertilised egg to become a female, and those with five, a male, of the free-living generation. No explanation has been offered of the fact that fertilised eggs with twelve chromosomes become females in one generation and hermaphrodites in the next, nor of what causes the different behaviour of the $X$-chromosome in the oogenesis and spermatogenesis of the hermaphrodite.

Another hermaphrodite species in which sex-chromosomes have been described is the Pteropod Creseis investigated by ZARniK. He found a difference between the oogenesis and spermatogenesis to some extent comparable with that observed in Rhabdonema, but his results are in some respects obscure, and until the case has been examined again it is hardly possible to accept them as final, and in any case the cytological basis for the production of male and female germ-cells by one individual remains unexplained. Further investigation of the whole subject is greatly needed. 
In all the cases mentioned hitherto the male differs from the female in producing two kinds of germ-cells, while the eggs are all alike, and the natural conclusion to be drawn is that in species which show this condition the sex of the individual developing from a fertilised egg depends upon the spermatozoon. For reasons that will be considered in a later chapter, however, it has for some years been known that at least in the Lepidoptera and Birds there must be male- and female-determining eggs, and it was suggested that in these groups of animals it was possible that the converse condition might be found-that the female might have an odd chromosome or an unlike pair of idio-chromosomes while the male had two equal sex-chromosomes. For some time the search for cases of this kind was unsuccessful, but in I9I3 SeIler discovered one in the moth Pbragmatobia fuliginosa. In this species he found 56 chromosomes in each sex, 54 autosomes and two large idiochromosomes, but in the maturation divisions the idiochromosomes behave differently in the two sexes. In the spermatocytes they pair and separate like the autosomes, so that all the spermatids receive 28 . In the anaphase of the first polar division of the egg, however, the two idiochromosomes behave differently. In the equatorial plate of this division they appear as a bivalent, as in the male, but in the anaphase one of them divides into two unequal parts, while the other, which goes to the opposite pole, remains as a single body. At the end of the first polar spindle, therefore, there are 28 chromosomes at one pole ( 27 autosomes and one large idio-chromosome, as in the male), and 29 ( 27 autosomes and a divided idio-chromosome) at the other. In some eggs the divided chromosome travels to the outer pole and enters the polar nucleus, in others it goes to the inner pole and is included in the egg-nucleus. There 
are thus two kinds of eggs, one with a complement of chromosomes like that of the spermatozoa, and one in which the idio-chromosome is divided.

A still clearer case was discovered shortly afterwards (1914) by the writer in the moth Abraxas grossulariata. In

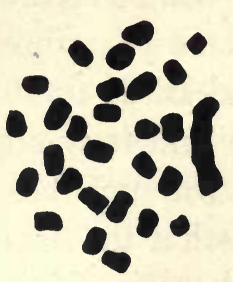

(1)

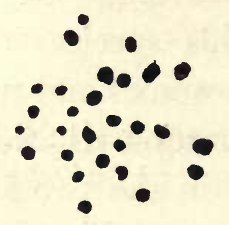

(3)

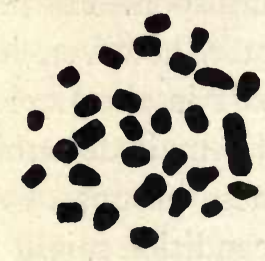

(2)

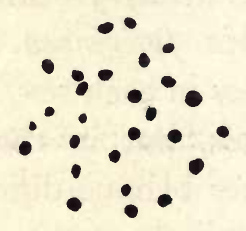

(4)

FIG. 19. Chromosomes in the maturation of the eggs of Moths. (Reproduced, by permission of the Editor, from Quart. Journ. Micr. Science, LIX (1 9 I 4), p. 506.) (I, 2) Phragmatobia fuliginosa, after SEILER. (I) Outer group of first polar division, 27 ordinary and one large chromosome (total 28). (2) Inner group of same egg, with 27 ordinary and one large chromosome divided (total 29). (3,4) Abraxas grossulariata, strain which has 55 chromosomes in the female before reduction. (3) Outer group of first polar division, 28 chromosomes. (4) Inner group of same egg, 27 chromosomes. In other eggs the outer group has 27 , and the inner group, from which the egg-nucleus is produced, 28.

this species, as in Phragmatobia, the somatic number is 56 in the male, and also in the majority of females, but in a strain which showed peculiar sex-ratios it was found that the females had only 55. The equatorial plates of the second polar division in this strain show clearly that one has 28 
and the other 27 , and as each kind of equatorial plate may be either the inner or the outer, there are evidently two kinds of mature eggs, some with 28 chromosomes in the eggnucleus and others with 27. Since all spermatozoa appear to have 28 , two kinds of fertilised eggs must exist, some with 56 and others with 55 , corresponding with the somatic numbers in the male and female of this strain ${ }^{1}$.

Up to the present time no other certain example has been found of females which produce two kinds of eggs differing in their chromosome number, but the two known examples are sufficient to make it clear that in the Lepidoptera at least this condition can exist ${ }^{2}$. If, therefore, we are justified in regarding sex as being determined by the presence or absence of a particular chromosome introduced into the zygote by one of the conjugating gametes, we must conclude that in a number of animals the presence or absence of this chromosome depends on whether it is or is not borne by the spermatozoon, but that in the Lepidoptera, and possibly some other groups, the spermatozoa are all alike, and that the differential chromosome is carried by the eggs.

1 Although it can hardly be doubted that the production of males and females in the strain of Abraxas in which the females have 55 chromosomes is usually dependent on the presence of either 28 or 27 in the egg-nucleus, a curious anomaly must be mentioned. Some females of a strain of this kind produce only female offspring, and yet the writer found that the eggs of these females may have either 27 or 28 chromosomes in the equatorial plate of the second polar division, so that, unless a chromosome is omitted in this division, for which there is no evidence, the egg-nucleus must contain sometimes 27 and sometimes 28 , although eggs of the same parents, reared to maturity, produced only females.

2 SeILER has recently (1917) described a third case in the Psychid moth Talaeporia in which the female has 59 chromosomes, the male 60 , and the mature eggs either 29 or 30 . 


\section{CHAPTER XII}

\section{Germ-cell Determinants}

$\mathbb{T}^{\mathrm{HE}}$ evidence given in the preceding chapter points

Strongly to the conclusion that in many animals sex is determined by, or at least is correlated with, the presence or absence of a particular chromosome in the fertilised egg, or, among parthenogenetic animals, in the egg after maturation. Before proceeding to consider whether other chromosomes may be regarded as determining the presence or absence of other inherited characters in the same sort of way, it will be convenient to make a short digression in order to describe how the separation of the germ-cells from the other cells of the organism may be dependent on the presence of definite bodies which are often known as "germcell determinants."

The conception of the "continuity of the germ-cycle" was introduced by WEISMAN , and was based largely on his researches into the origin of the germ-cells of Diptera and Hydrozoa. The essence of the idea, at least in its original form, was that the germ-cells are not produced by the body, but exist as an independent cycle, continuous from generation to generation, living in and nourished by the body, but not formed from it. According to this conception, when an egg segments, some cells are from the first marked off from the rest as primitive germ-cells; they remain undifferentiated while the other embryonic cells become modified into the various tissues, and when the organism is adult they give rise to ova or spermatozoa. The fertilised egg thus 
gives rise on the one hand to the body or soma, and on the other hand to the cells of the "germ-track" (Keimbabn); the body grows, becomes adult, and finally decays and dies, but the germ-cells have a sort of potential immortality, giving rise to similar germ-cells, as well as to a body, in the next generation.

This idea, in its original simple form, was soon modified in consequence of such phenomena as budding and regeneration, in which it was clear that definitely somatic cells could give rise to complete new individuals or parts, and in place of the distinction between soma and germ-track WeIsmann substituted that of somato-plasm and germplasm, and supposed that although the somato-plasm or body-plasm could never be converted into germ-plasm, yet ordinary body-cells might retain sufficient germ-plasm to enable them to regenerate lost parts or even to produce a complete individual. It is not our purpose to discuss any further this speculation of WeIsmann's, which, whether true or false, has had very great influence on the biological thought of the past thirty years, but rather to consider some of the cases in which the differentiation of body-cells from germ-cells may actually be observed.

In a considerable number of animals, belonging to different groups, at an early stage of embryonic development certain cells are recognisable which remain distinct from the surrounding cells and ultimately give rise to the gonads. Cells of this kind have been known for many years in Sagitta, in certain Nematodes and Insects, and in some other forms, but only more recentiy has it been shown that they are cytologically different from the neighbouring cells which give rise to the body tissues. One of the earlier cases in which this was demonstrated was the embryo of the Nematode Ascaris megalocepbala investigated by 
BOVERI (1903 a). In the variety univalens of this species there are two long chromosomes in the fertilised egg, each of which has the ends somewhat thickened. In the first segmentation division each of these divides longitudinally in the ordinary way, giving rise to two cells each with two long chromosomes in the nucleus. In the second division, however, the two cells behave differently; in one the two chromosomes divide as before, but in the other they each break up into a string of small elements, and throw off the swollen ends (Pl. XXI). The small bodies into which the middle part of the chromosomes have broken up divide like ordinary chromosomes; the cast-off ends do not divide but become included irregularly in the two daughter-cells when the cell divides. In the four-cell stage there are thus two cells with large nuclei containing the complete long chromosomes, and two cells with smaller nuclei in which "chromatindiminution" has taken place. In the next (third) segmentation division the numerous small chromosomes appear and divide in the two cells that have undergone diminution; of the other two cells, one retains the two full-sized chromosomes and the other in its turn undergoes diminution, the chromosomes breaking up into numerous small elements and throwing off their ends. In the fourth and fifth divisions the same process is repeated (i.e. four times in all), so that in the equatorial plate of the division between the sixteen and thirty-two cell stages fourteen cells have already the "diminished" chromosomes, in one the chromosomes are undergoing diminution, and in the sixteenth the chromosomes remain as they were in the fertilised egg. This last cell is the primitive germ-cell; its descendants undergo no further diminution, and from them only the gonads are produced, while the remaining fifteen cells give rise to the rest of the body. 

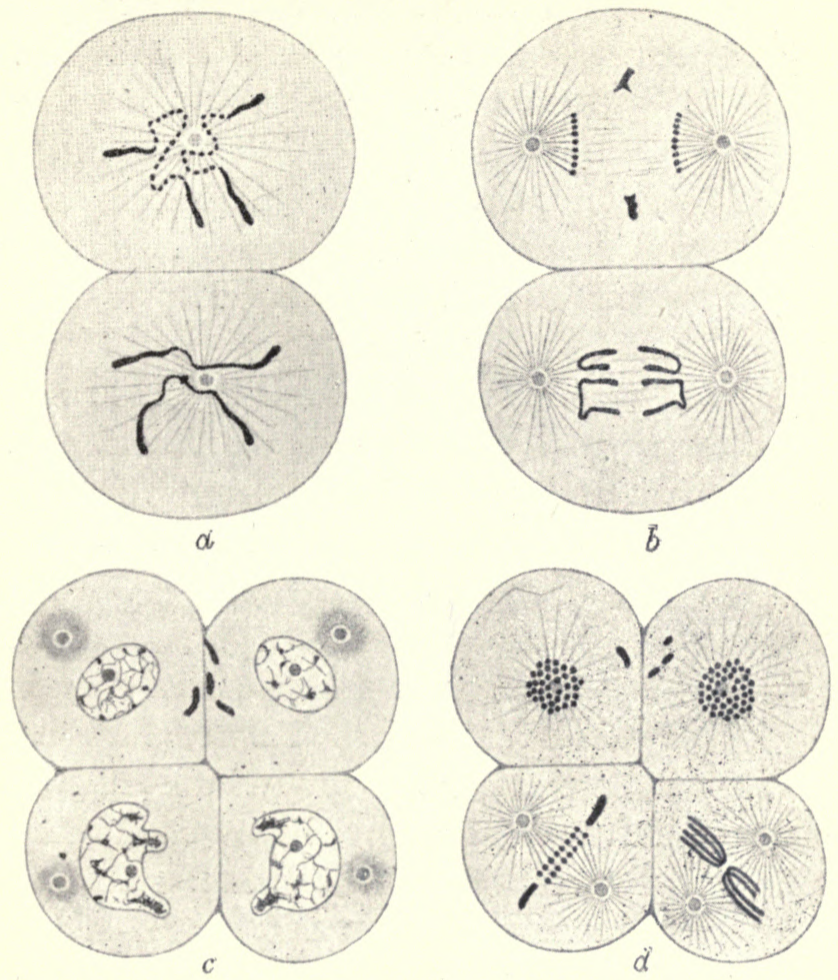

Four stages of the segmentation of the egg of Ascaris megalocephala univalens, somewhat diagrammatic, after BOVERI ( $1903 a$ ).

a. Close of first division; in the upper cell the long chromosomes are breaking up into short units and throwing off the thickened ends.

b. Anaphase of second division; in the upper cell the thickened ends of the chromosomes take no part in the mitosis.

c. Four-cell stage with resting nuclei.

d. Metaphase of third division, in the upper cells in face, in the lower cells from the side. The left lower cell is undergoing "chromatin diminution." 

Somewhat similar chromatin-diminution has been found by later investigators in other species of Ascaris (for example, $A$. lumbricoides), but it has not been observed in a number of other Nematodes which have been examined. Although the whole of the chromatin is retained in the cell which will give rise to the germ-cells, while some of it is extruded from the nucleus in the other cells, experiments (such as centrifuging the eggs during development) show that some cytoplasmic factor determines whether diminution shall occur or not, for in eggs which have been centrifuged or are otherwise abnormal two cells may retain the full complement of chromatin and both become primitive germ-cells. The case may thus be compared in this respect with the process that determines sex in Pbylloxera, where the presence or loss of a particular chromosome in the egg determines the sex of the individual which develops from it, but some pre-existing factor decides whether the chromosome shall be extruded or not. Phenomena in some ways comparable with the chromatin-diminution of Ascaris are found in the developing oocytes of certain Insects, but before describing this it will be convenient to consider other cases of bodies which appear to determine the origin of the primitive germ-cells in the early embryo.

One of the classical examples of the very early differentiation of the germ-cells is the little marine organism Sagitta, the systematic relationships of which are still quite obscure. In the adult the coelom is divided towards the hind end of the body by a transverse septum, and a longitudinal septum separates each portion into right and left halves. Just in front of the transverse septum on each side lie the two ovaries, and the testes are in corresponding positions behind it. Each ovary and testis is derived from a single cell, and these four cells are formed by the division of two 
cells which are visibly differentiated from the rest of the embryo in the gastrula stage. Lastly, these two primitive germ-cells are derived from a single cell of the 32-cell stage, so that this one cell gives rise to the gonads and to nothing else. To describe the origin of this cell it will be best to begin with the egg at the time of fertilisation and trace its development, as it has been worked out by Elpatiewsky (I9Io), Miss Stevens (I9Iob) and Buchner (I9I0). At the stage when the male and female pronuclei are approaching conjugation, a body appears at the edge of the egg (usually called Elpatiewsky's "besonderer Körper"), consisting at first of a mass of granules which stain deeply and which later condense to a homogeneous stained mass. The origin of this body is unfortunately uncertain. Elpatiewsky and Miss Stevens find no trace of it at an earlier stage, while BUCHNER maintains that it is derived from the nucleus of an accessory cell which is concerned in guiding the spermatozoon to the egg, and which, he supposes, enters the egg protoplasm and gives rise to the "besonderer Körper." The two former investigators, however, give good reasons for believing that BUCHNER is mistaken, and the only other suggestion is that the body is derived from chromatin granules which are extruded from the oocyte nucleus at a rather earlier period, although, if this is so, the intervening stages have not been found. Whatever its origin, the "besonderer Körper" does not divide in any of the first five segmentation divisions of the egg, with the result that at the 32 -cell stage it is confined to one cell, and this becomes the primitive germ-cell (P1.XXII, upper four figures). When this cell divides, the body breaks up into several pieces, some of which pass into both daughter-cells, but according to Elpatiewsky and to Miss Stevens the distribution is uneven, one cell receiving more than the 


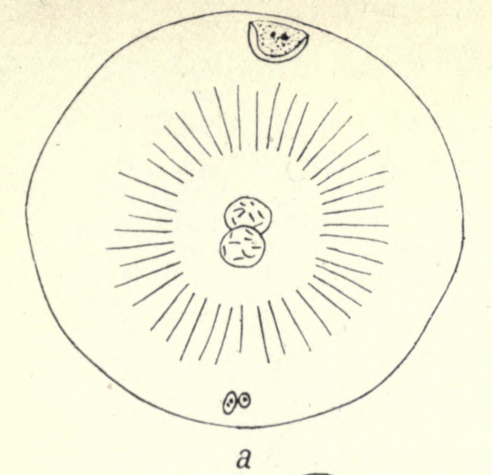

Plate XXII
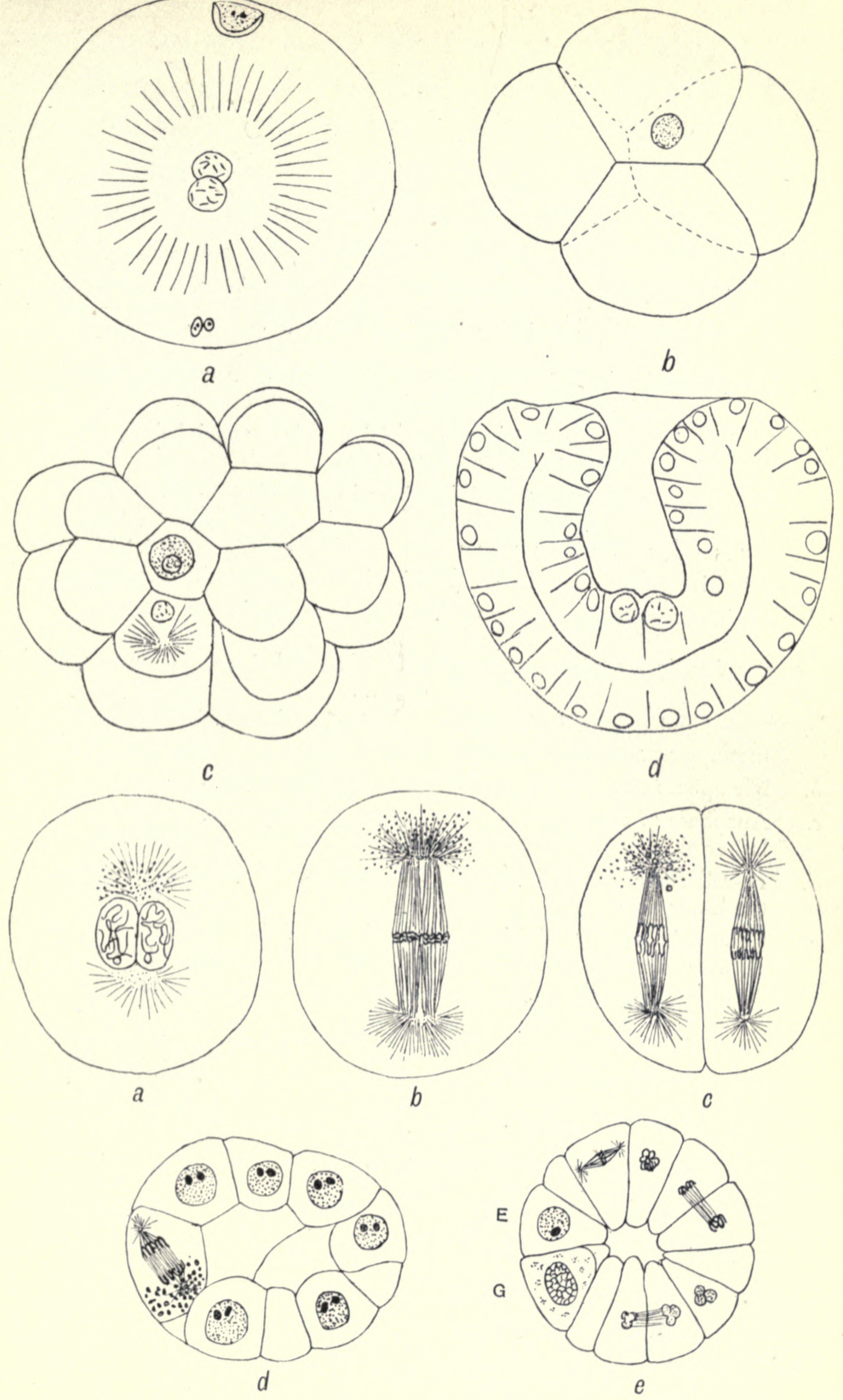


\section{Explanation of Plate XXII.}

Upper four figures. Stages in the development of the egg of Sagitta.

Slightly modified from Elpatiewsky (1 909).

a. Conjugation of egg and sperm nuclei. Polar nuclei below, germ-cell determiner ("besonderer Körper") above.

b. Four-cell stage-germ-cell determiner in one blastomere.

c. 32-cell stage. The cell of the 16 -cell stage which contained the germcell determiner has divided into two cells, an upper which forms the primitive germ-cell and a lower which is the first primitive endoderm-cell.

d. Section of gastrula with two primitive germ-cells in archenteron.

Lower five figures. Stages in the fertilisation and segmentation of Cyclops, after Амма.

a. Sperm and egg nuclei in contact. Beginning of first cleavage spindle and first appearance of ectosomes.

b. Metaphase of first cleavage; ectosomes at upper pole.

c. Anaphase of second cleavage; ectosomes at upper pole of one cell. The larger granules are ectosomes persisting from the preceding division.

d. I 5-cell stage, $4^{\text {th }}$ division of the granule-containing cell. Ectosomes at the lower pole.

e. Completion of $4^{\text {th }}$ division of granule-containing cell into a lower cell, $G$, with granules, which forms the primitive germ-cell, and an upper cell, $E$, which gives rise to the endoderm. The other blastomeres are undergoing their fifth division. 
other. They suppose that this division is a differential one, and that one of the cells formed by it gives rise to both the testes, the other to the ovaries. Here again BUCHNER disagrees, and maintains that the separation of the primitive spermatogonium from the primitive oogonium takes place at the next division, when the two primitive germ-cells divide into four, but on the whole Miss Stevens' evidence points more strongly to the former opinion. In Sagitta, therefore, as in Ascaris, a single primitive germcell is differentiated at an early stage of segmentation by the presence of a body that is absent from the other cells, but in Sagitta the body is of unknown origin and not certainly chromatinic, while in Ascaris it consists in parts of the chromosomes.

A third case of the very early differentiation of a primitive germ-cell is provided by the paedogenetic eggs of Miastor, a fly of the Dipteran family Cecidomyidae. The main outlines of the process were observed more than fifty years ago by Leuckart and Metschnikoff, and more recently by Kahle (1908) in Europe, and Hegner (19I4) in America. Miastor is remarkable in having larvae which reproduce viviparously by a process of parthenogenesis ("paedogenesis"). The mature larvae have two ovaries, each containing typically thirty-two oocytes, though the number may be smaller owing to the suppression of some of them. The oocytes are peculiar in having attached to them a group of nurse-cells that are not derived, as in most other insects from oogonia, but are immigrant mesoderm cells. At the end of the egg opposite the nurse-cells is a patch of protoplasm called by HeGner the pole-plasm. The egg-nucleus undergoes a single, non-reducing polar division, and the mature nucleus then begins the segmentation mitoses in the central yolky portion of the egg. When two divisions have taken

D. c. 
place, one of the four nuclei produced enters the pole-plasm at the posterior end of the egg, and in the next division its behaviour differs from that of theother three(P1. XXIII). In the division of the three nuclei in the yolk a process of chromatin-diminution takes place. Parts of each chromosome, or possibly some complete chromosomes-the descriptions are not explicit on this point-are left behind in the equatorial region of the spindle in the anaphase, so that the daughternuclei contain only a portion of the chromatin that was present in the parent nucleus. The fourth nucleus, situated in the pole-plasm, meanwhile undergoes a normal mitotic division without diminution, and of the two nuclei produced from it one remains in the pole-plasm and the other moves forward into the yolk. At the next (fourth) segmentation division each of the nuclei in the yolk, including the sister-nucleus of that in the pole-plasm, again undergoes chromatin-diminution, and according to KAHLE in their subsequent divisions these nuclei show only half the number of chromosomes that are present in the germ-cells. At the stage when eight nuclei are present the pole-plasm with its included nucleus is cut off from the rest of the egg as a separate cell, which does not divide again immediately. This is the primitive germ-cell, and in its subsequent divisions it always retains the full amount of chromatin. It later divides into two, and then into four and eight, and these eight cells group themselves in two rows of four, one on each side of the embryo, to give rise to the ovaries. Then by three more divisions the number of cells in each ovary is increased to thirty-two, which is the number of oocytes in the mature ovary. It is remarkable that the primitive germ-cell gives rise to nothing but germ-cells, for the nurse-cells originate from the enclosing mesodermal envelope; also that the total number of cell-divisions from 

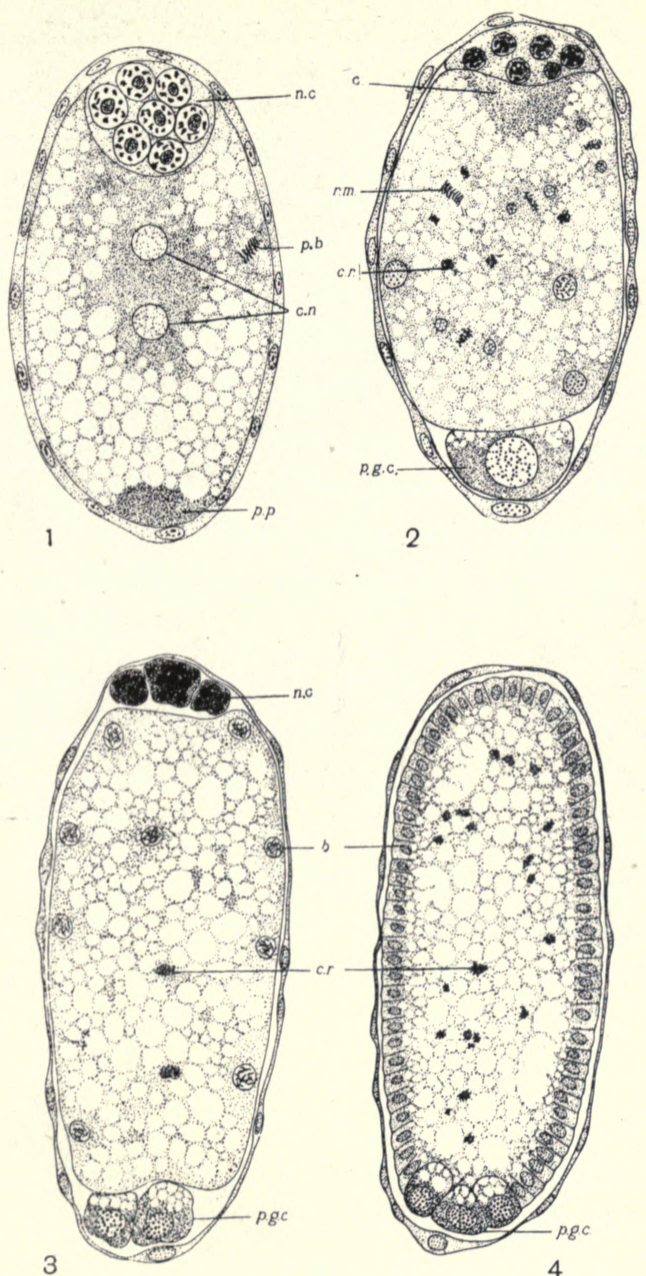

Segmentation of the egg of Miastor americanus, after Hegner (1 914 ). I, two segmentation nuclei (c.n.), dividing polar nucleus ( $p . b$.$) , polar plasma$ (germ-cell determiner, p.p.), and group of nurse-cells (n.c.).

2, rather later stage with one primordial germ-cell (p.g.c.), nuclei undergoing chromatin-diminution (r.m.), and clumps of cast-out chromatin (c.r.). 3 , 4, early and later blastoderm stages with two and four primordial germ-cells. 

its separation to the production of the full-grown oocytes seems always to be six.

The case of Miastor is interesting from the fact that it includes a process of chromatin-diminution in the bodycells, but not in the germ-cells, much as in Ascaris, but that the determining cause of the development of one nucleus into a germ-cell seems to be its association with the visible pole-plasm at the hind end of the egg. A similar pole-plasm, or a group of staining granules which corresponds with it, has been observed in a number of other Diptera, and as in Miastor those segmentation nuclei which come into relation with it become the primitive germ-cells. Miastor is, however, the only insect in which it has been observed that the body-cells are distinguished from the germ-cells by chromatin-diminution.

Very similar phenomena to those found in the Diptera other than Miastor have been described by Hegner in the Chrysomelid Beetles Calligrapha and Leptinotarsa. In these there is a group of granules at the posterior end of the egg, and when, after repeated segmentation divisions, some of the nuclei come to the surface to form the blastoderm, four nuclei come into relation with these granules, and instead of taking part in the general blastoderm formation, they push their way through the egg-surface and form a group practically outside the egg. As they do this they take some of the protoplasm and the granules with them, and by two successive divisions give rise to sixteen cells, which are the primitive germ-cells. It appears to be a matter of chance which of the segmentation nuclei come into connection with the granules and so give rise to germ-cells, and HEGNER showed that by destroying the granules with a hot needle in the fresh egg, embryos without germ-cells were produced. 
A germ-cell determinant of a different kind has been described by Silvestri and others in the parasitic Hymenopteran Litomastix (Copidosoma). It consists of a deeplystaining compact body lying in the egg before maturation, called by Silvestri the oosoma (Text-fig. 20). The egg is very small, and undergoes total segmentation (very rare in Insects) and the stained body remains undivided and is enclosed in one cell at the four-cell stage. It then enlarges and breaks up, so that when this cell divides both daughter-cells will contain part of it. As these cells divide, always containing portions of the stained body, they form a mass at the anterior end of the embryo. This mass then breaks up into a number of cell-groups or morulae, some of which again divide in the same way. Ultimately each of the morulae develops into a larva, so that one egg gives rise to scores or hundreds of larvae. The larvae are said to be of two kinds; some are normal Hymenopterous larvae with all the usual organs, including gonads; others are "sexless" larvae with no gonads and reduced alimentary canal. The sexless larvae never develop to adults, and Silvestri believes that they are formed from groups of cells which have received no portion of the stained body of the egg. In related species in which polyembryony does not occur it seems almost certain that a similar stained body determines the cells which will become primitive germcells. Of the origin of the stained body little is known; Silvestri's supposition that it is the nucleolus extruded from the egg-nucleus, and HEGNER's, that it is derived from the chromatin of a degenerate oocyte, have both been disproved, and it seems more probable that it is a portion of modified and concentrated protoplasm, as in the Diptera and Coleoptera.

The early differentiation of primitive germ-cells has also 

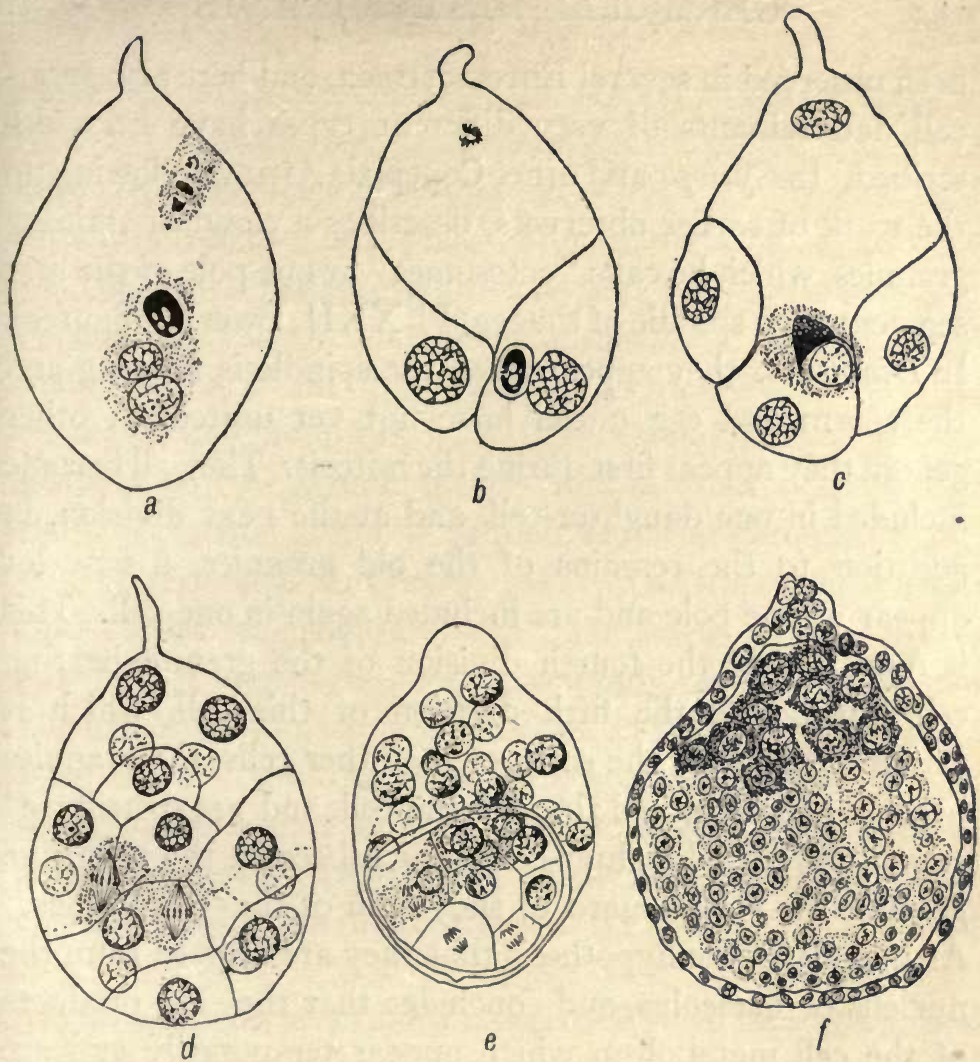

FIG. 20. Stages in the development of Litomastix, after Silvestri (1906).

a. Conjugation of egg and sperm nuclei. The dark vacuolated body is the " oosoma," and near the upper pole of the egg are the three groups of polar chromosomes.

$b, c$. Two- and four-cell segmentation stages. The polar chromosomes unite into a single nucleus and the oosoma passes undivided into one daughter cell $(b)$ and begins to break up $(c)$.

d. Fourteen-cell stage : the polar nucleus has divided into four; the oosoma has broken up and its fragments are contained in two cells which lag behind the others in division.

e. Twenty-cell stage: the fragments of the oosoma are contained in four cells; the polar nucleus has divided into sixteen.

$f$. Later stage. The polar nuclei and the protoplasm surrounding them have given rise to an envelope (trophamnion) surrounding the embryonic mass. The latter consists of larger cells at the anterior and smaller cells at the posterior end. The larger cells are probably derived from those containing fragments of the oosoma. 
been observed in several Entomostraca, and here also germcell determinants of very different types have been described. In Cyclops and other Copepods Amma, following up the work of earlier observers, describes a cloud of staining granules, which he calls "ectosomes" at one pole of the first segmentation spindle of the egg(Pl.XXII, lower five figures). In Diaptomus they appear when the spindle is forming and the sperm and egg nuclei have not yet united, in other genera they appear first during the mitosis. They all become included in one daughter-cell, and at the next division, in addition to the remains of the old granules, a new lot appear at one pole and are included again in one cell. This is repeated till the fourth division of the granule-bearing cell but before the fifth division of this cell, which is simultaneous with the sixth of the other cells, the granules become evenly spread through the cell, and are distributed equally to both daughter-cells, which become the primitive germ-cells. With regard to the origin of the "ectosomes," Aмma rejects the hypothesis that they are derived from the nucleus or nucleolus, and concludes that they are products of the cell metabolism which appear temporarily and are then re-absorbed.

In the Cladoceran Polyphemus, Küн (19I3) finds that a nurse-cell enters the egg before cleavage; in the fourth segmentation division the blastomere containing it divides into two cells, of which the one which includes the now fragmented nurse-cell becomes the primitive germ-cell and the other the first endoderm cell. In the succeeding divisions of the primitive germ-cell, the remains of the nurse-cell are distributed to both daughter-cells. In other Cladocera which have been examined for comparison no similar germ-cell determinant has been discovered.

From the examples described it will be seen that the so- 
called germ-cell determinants are of very different types in different animals, and that their occurrence appears to be very irregular, since they may be present in one genus and absent in others that are nearly related to it. In some cases the difference between germ-cells and body-cells seems to depend on the presence of chromatin in the germ-cell nuclei which is lost from those of the body-cells, but the extrusion of chromatin, when it occurs, appears to be dependent on cytoplasmic factors, and in other animals only the cytoplasmic factor is present and the chromatin content of germ-cells and body-cells is to all appearance alike. And of the real nature of the cytoplasmic factor, hardly anything is known; its existence is only certain when some portion of protoplasm, or of bodies in it, stains differently from the rest, and the apparently great difference between related species may be due, in some instances at least, to a mere difference of staining capacity of the protoplasm concerned with the differentiation.

The examples mentioned hitherto have all been concerned with the very early differentiation of germ-cells in the embryo, but other cases have been observed where a visible cytological differentiation occurs at a much later stage. Only two of these will be described. One of the best known is that of the separation of the oocyte from the nurse-cells in the Beetle Dytiscus, described by Grardina and subsequently by Debaisieux and by Günthert. After a series of typical oogonial divisions, their nuclei undergo a curious differentiation; the chromosomes appear in one half of the nucleus while the other hemisphere is filled with staining granules which, according to GÜNTHERT, are produced by separation of fragments from the chromosomes, though Debaisieux regards them as of nucleolar origin. The mass 
of granules becomes arranged in a crescentic form around the nucleus, and when the cell divides the whole of this crescent passes to one pole, where it surrounds the reconstituted
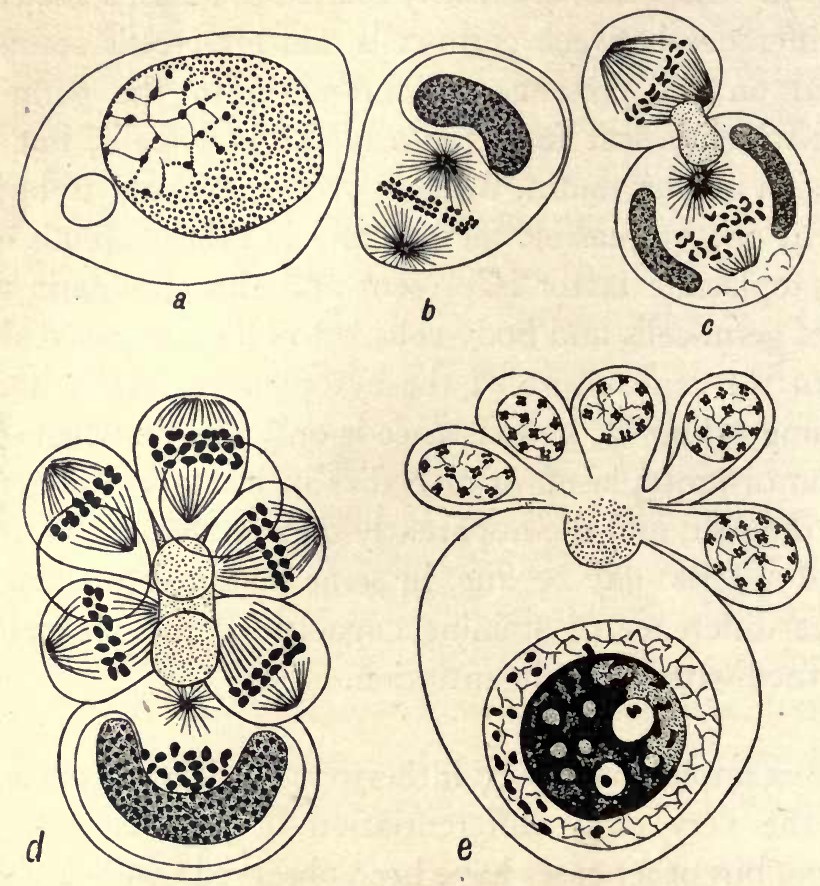

FIG. 2 I. Stages in the development of oocytes and nurse-cells in Dytiscus, after GüNTHERT.

a. Oogonium with cresent-shaped mass of granules in nucleus.

b. First differential division; the granules form a crescent at one pole.

c. Second differential division, metaphase; the granules encircle the spindle.

d. Fourth differential division.

e. Growing oocyte after completion of fourth differential division. Only five of the fifteen nurse-cells are seen in the section.

nucleus of the daughter-cell. The cell which receives it is that which contains the "Spindelrestkörper" of the preceding division. Both cells then divide again, that with the crescent or "chromatic ring" of GIARDINA again,under- 
going a differential division, and the process is repeated in all four times, giving rise to a group of sixteen cells, of which one contains the chromatic ring and the other fifteen are without it. All the cells remain connected together by short

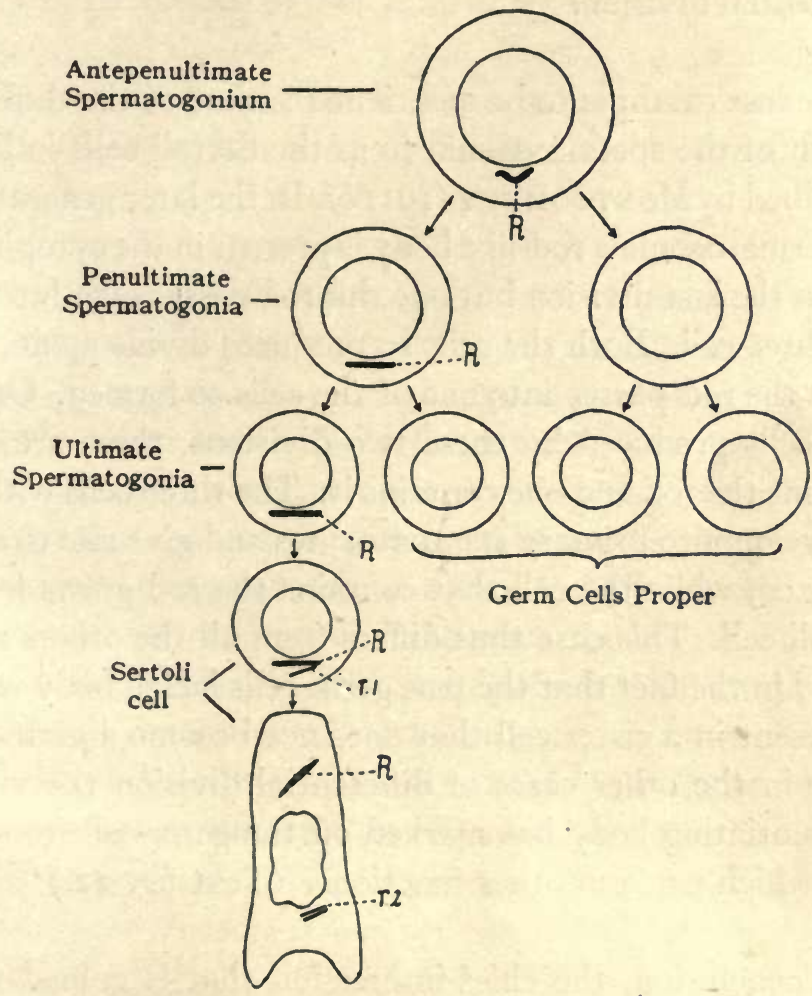

FIG. 22. Diagram of differential division of a Sertoli cell from spermatogonia in Man, after Montgomery ( $1911 b$ ).

stalks, due to incomplete division. The cell with the chromatic ring is the oocyte, and the fifteen others become nutritive cells which supply nourishment to the oocyte during its subsequent growth. In the later oogenesis of Dytiscus, therefore, as in Ascaris and Miastor, a true germcell is separated from cells that will not become germ-cells 
by possessing chromatin which is absent from the others, but in this case the nutritive cells do not simply extrude chromatin which is lost, but all of it goes to the germ-cell by means of what BoverI calls a more economical type of differential division.

The last example to be mentioned is that of the differentiation of the spermatogonia from the Sertoli cells in Man, described byMontgomery (I9I I $b$ ). In the later generations of spermatogonia a rod-like body is present in the cytoplasm, and in the last division but one this rod passes over into one daughter-cell. Both the cells so produced divide again, and again the rod passes into one of the cells so formed. Of the four cells produced by these two divisions, three are thus without the rod and one contains it. The three cells without it develop into primary spermatocytes and give rise to spermatozoa, while the cell that contains the rod grows into a Sertoli cell. This case thus differs from all the others mentioned in the fact that the true germ-cells lack a body which is present in a sister cell that does not become a germ-cell, while in the other cases of differential division the visible differentiating body has marked off the germ-cell from the cells which perform other functions. (Text-fig. 22.)

In conclusion, the chief impression that is gained from the comparative study of "germ-cell determinants," at least when they are present at the beginning of development and become confined to the cells of the germ-track by differential division, is that although these bodies are evidently strictly correlated with the germ-cells, there is no absolute certainty that they are the cause of the differentiation of germ-cells from body-cells. In Miastor, for example, the germ-cells do not undergo the chromatin-diminution that is character- 
istic of the body-cells, and yet the cause of the difference seems rather to lie in the pole-plasma than in the additional chromatin. In Ascaris, although no visible cytoplasmic factor is present, the experimental evidence points to a similar conclusion, and it may therefore be important to keep in mind the possibility that a body may be a constant feature of the germ-cells as distinguished from body-cells, and yet not be the cause of the difference in function and behaviour.

Gatenby's suggestion in his work on Trichogramma (1918b) that "the function of the determinant is nutrimental" may well be true of similar bodies in other forms. If so, it may be supposed that the various forms of germ-cell determinants are at least usually either themselves masses of reserve material, or are the visible expression of the existence of supplies of such material in the cells, which result in delaying or preventing cell differentiation, and so cause the cells to remain in an embryonic condition. The body-cells would thus undergo differentiation, while the cells containing the "germ-cell determinant" would remain in their primitive unmodified condition, and so be capable at a later stage of giving rise to germ-cells ${ }^{1}$.

1 Gatenby (1918c) concludes that in the polyembryonic Hymenoptera (Litomastix, etc.) there is no evidence of a "germ-track," and that Silvestri"s oosoma " has no other effect than that of temporarily stopping mitosis in the cells which happen to contain it." But in these species the egg divides at the beginning of development into an anterior part which forms the embryonic envelope, and a posterior part which forms the cells that give rise to the numerous embryos (Fig. 20; cf. also Fig. 10, p. 82). This suggests the speculation that the posterior part may perhaps be regarded as a single primitive ovum, which divides into many ova, and that these then undergo parthenogenetic development into larvae. If this were true, the polyembryony of such forms as Litomastix could be correlated with the alternation of sexual and parthenogenetic generations in the Cynipidae. 


\section{CHAPTER XIII}

\section{The Theory of the Individuality of the Chromosomes}

$T^{T}$ is now necessary to return to a further consideration of the behaviour and functions of the chromosomes, from which the subject of germ-cell determinants was to some extent a digression, and in particular to discuss a theory which may be regarded as one of the broadest and most fundamental generalisations of modern cytology. This theory, due very largely to the late Prof. Boveri, is now very widely, but by no means universally, accepted, and is the foundation of all the more recent hypotheses and speculations on the function of the chromosomes in relation to the transmission of hereditary characters. Put quite shortly the theory of the individuality of the chromosomes states that the chromosomes behave in a certain sense as individuals, remaining in existence from one nuclear division to the next, during the so-called resting stage of the nucleus, and dividing in mitosis to form two new individuals each exactly like the parent chromosome from which they originated. Stated in an extreme form, the theory maintains that the chromosomes behave like independent organisms living in a sort of symbiosis with the rest of the cell, deriving their substance from it and secreting substances that are used by it, but remaining as distinct from it as the "Zooxanthellae" in the body of a Radiolarian. Such a statement must of course be regarded as metaphorical, and rather as illustrating the kind of individuality that is meant than as describing the condition as it is supposed actually to exist, but even with this 
provision the most convinced adherents of the individuality theory no longer hold it in this extreme form. For in what has gone before it has already been seen that in several cases the chromosomes cannot be regarded as individuals in any such sense, for bodies which at some stage behave as one chromosome may sometimes break up at another stage into several or many smaller units. A conspicuous example of this is seen in the segmentation of the eggs of Ascaris, in which the two long chromosomes of the fertilised egg not only throw off their thickened ends, but also break up into many small portions, in the cells which do not give rise to the germ-track. Similar division of chromosomes that at some stage behave as units has been observed in several animals, and other reasons for regarding the chromosomes as compound will be considered in the chapter on their relation to hereditary characters.

In consequence of facts of this nature, the original individuality hypothesis has been modified in such a way as to assume that the chromosomes as they ordinarily appear are not indivisible units, but are composed of smaller portions to which the theory of individuality applies strictly. According to this view the chromosomes are definite aggregations of individual units arranged in a constant manner; the units persist as individuals from one mitosis to the next during the resting stage, and since they are arranged along the chromosomes in a definite order, when the chromosomes divide in the mitotic figure, their component units are similarly divided, so that each daughter chromosome is composed of a similar series of individual units. Although, therefore, the units are the ultimate individuals, the chromosomes themselves have also a definite individuality, for they are composed of a constant series of these smaller individuals. As a rough analogy, the units may be com- 
pared to the men of which a regiment is composed, and the chromosomes to the regiment. The regiment commonly acts as a whole, and has a definite corporate individuality of its own, but is made up of many indivisible units, which may on occasion be replaced by others, and to which the word individuality in its strict sense can be applied ${ }^{\mathbf{1}}$. It now remains to consider the evidence upon which this theory rests.

The theory of chromosome individuality is based on a number of different and often quite independent arguments, most of which are due very largely to Boveri, who set them forth in a famous paper in 1903 (see bibliography). Since then many more recent discoveries have confirmed and amplified some of his arguments, and some new ones have been added, while very few of the facts and ideas which he brought forward in support of the theory have been seriously challenged. The discussion that follows will therefore be based to a considerable extent on Boveri's paper, although more recent examples will be used when they seem better adapted to illustrate the matter under discussion. The lines of argument may be arranged most conveniently under separate headings.

(I) The definite number of chromosomes in each species.

In general, though with significant exceptions, the chromosome number is constant not only in all the cells of an individual, but in all individuals of a species. This fact, without in any way proving their individuality, certainly makes it more probable, for if the chromosomes behave as individuals the constant number is a logical necessity, while otherwise it must be assumed that some innate characteristic of the species causes the chromatin of the nucleus to concentrate itself into the same number of portions at each mitotic

1 This analogy of the chromosomes to regiments of men is due originally to FICK, and is a modification of his well-known "Manövrier-hypothese." 
division. And the various kinds of exceptions to the rule provide strong arguments in favour of the individuality hypothesis, for all, or nearly all, are easily explained by its aid, but are quite inexplicable otherwise. For example, Boveri points out that occasionally in Ascaris megalocephala univalens an abnormal polar division may leave two chromosomes in the mature egg instead of one. Such an egg, fertilised by a normal spermatozoon with one chromosome, contains three chromosomes in its zygote-nucleus. If the chromosome number were determined by some inherent
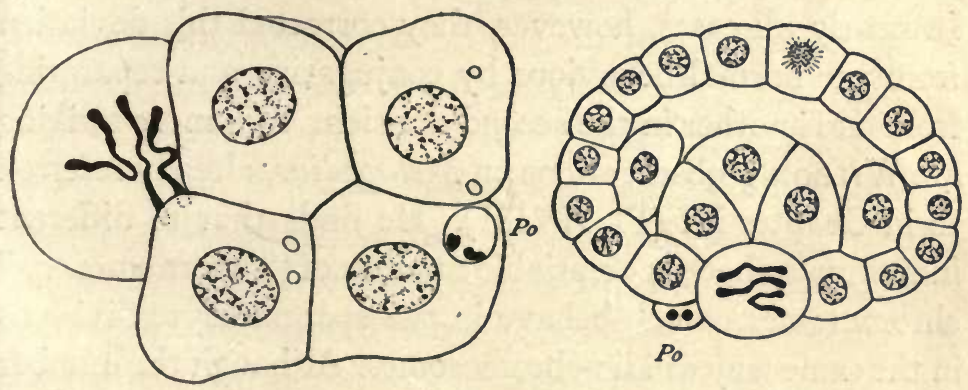

FIG. 23. Segmentation of a three-chromosome egg of Ascaris, showing that the abnormal chromosome number persists to the gastrula stage. The large cell in the later embryo is the primitive germ-cell; Po, the polar body. From Boveri (1903 a).

character of the species, it would be expected that in the later segmentation divisions the chromatin would be aggregated into the two chromosomes which are characteristic of normal embryos of this species, though each might perhaps be rather larger than usual. But in fact it is found that such eggs continue to show three chromosomes in the cells of the germ-track (which do not undergo diminution) up to the end of the segmentation stages; when once the number has become abnormal through any cause, it remains so although the development is otherwise typical (Text-fig. 23). 
A similar and still more striking instance of the same sort of thing will be described in a later chapter when discussing "non-disjunction" of chromosomes in Drosophila.

Other examples of abnormal behaviour of chromosomes causing a departure from the typical number, but supporting the individuality hypothesis are provided by the observations of Montgomery on Euschistus (1910) and of Wilson on Metapodius (1909), two genera of Hemiptera. In Euschistus Montgomery found that not very rarely a pair of chromosomes failed to conjugate in the first spermatocyte division, but remained separate and divided equationally. In nearly all cases, however, they corrected this deviation from the normal behaviour by conjugating and separating from one another in the second division. Still more striking are WiLson's observations on Metapodius; already referred to in Chapter XI (Pl. XVIII). He finds that in different individuals there is a variable number of "supernumerary" chromosomes, which behave in the spermatocyte divisions in the same way as idio-chromosomes. Although the number varies in different specimens, it is constant in the cells of any one specimen, and all the various numbers found can be accounted for on the assumption of the occasional passing of the $r$-chromosome to the same pole as the $X$, giving rise to a spermatozoon with an extra chromosome, if it is conceded that this chromosome retains its individuality. Furthermore, there is in Metapodius one pair of very small chromosomes, and in all the sperm-cells of one specimen WiLson found that instead of a normal supernumerary there was an extra chromosome which in the first spermatocytes united with this pair and passed with one of them to the pole. In appearance it was indistinguishable from an ordinary supernumerary, but its different behaviour proved its individuality. 
Again, the persistence of the reduced number of chromosomes in the cells of parthenogenetic embryos is an example, of a somewhat different kind, of the retention of an abnormal number when it has once been produced. In this case, however, in some animals at least the normal (diploid) number is restored in some of the tissues, probably by a division of the chromosomes without nuclear division. In some male Hymenoptera, for example, the haploid number persists in the germ-cells, and, at least to a late larval stage, in the cells of the nervous system, while in other somatic cells the diploid number is restored.

Instances of abnormality in chromosome number in the opposite direction are afforded by so-called "tetraploid" races of animals and especially plants, in which all the nuclei contain twice as many chromosomes as are characteristic of the species. In a number of plants (Primula, Oenothera, etc.) races are known in which the chromosomes have double the normal number; frequently such plants are giants, in consequence of the large size of their cells, and often also they are apogamous, that is, their ovules develop parthenogenetically without requiring fertilisation. This apogamy of tetraploid plants may be compared with the condition already referred to in Artemia, in which the race with 84 chromosomes is parthenogenetic, while that with 42 reproduces sexually. In the banana three races have 8, i 6 and 24 chromosomes respectively in their nuclei, and in different species of Chrysanthemum the (haploid) numbers 9, I $8,27,36$ and 45 have been recorded ${ }^{1}$. WINGE points out that in plants $6,8,9,12$ and 16 are much the commonest haploid chromosome numbers, and that the next most frequent numbers are simple multiples of these. All these facts suggest that the chromosomes are to some extent individuals,

- 1 Tahara and Tischler, quoted by Winge (1917).

D. C. 
and that changes from one number to another occur either by reduplication or by nuclear fusion not followed by a "reducing" division.

The examples of various kinds of numerical abnormality given above thus suggest that the chromosome number is dependent on the behaviour of the chromosomes as individuals, and not on some innate tendency for the chromatin of the nucleus to aggregate itself into a definite number of masses during mitosis, while ceasing to have any such arrangement in the resting condition of the nucleus.
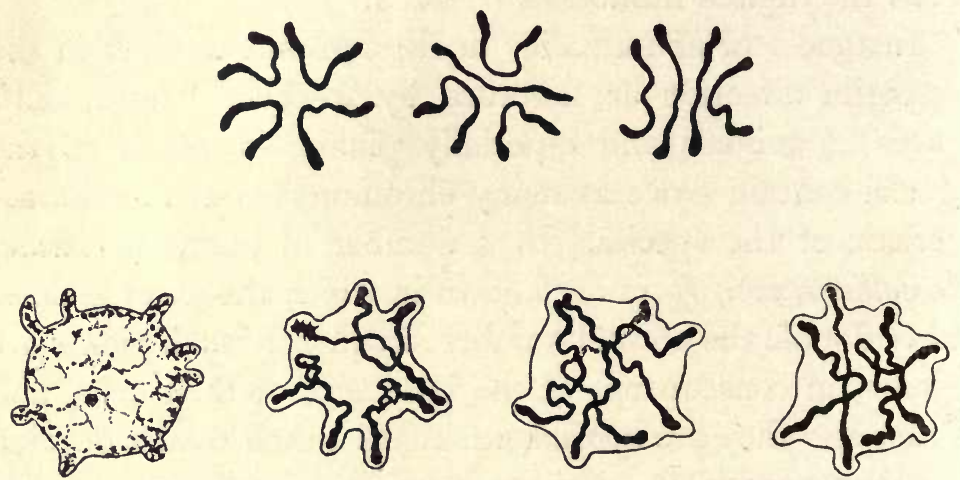

Fig. 24. Ascaris megalocephala bivalens. Above, three equatorial plates of first segmentation division showing different arrangements of chromosomes. Below, nuclei of two-cell stage in reticular condition and in preparation for the next division. From BOVERI (1903 a).

(2) The persistence of definite chromosomes in the resting stage of the nucleus.

Generally speaking the chromosomes are only visible as definite bodies during mitosis, and one of the chief objections to the individuality hypothesis is that not only do they disappear in the reticular stage of the nucleus, but observation seems to show that in this stage the chromatin becomes more or less uniformly distributed over the network, and that the chromosomes, as such, cease to exist. Careful 
observation of nuclei, however, especially in the telophase and early prophase of mitosis, has provided evidence that in some organisms the chromosomes, or at least the framework or basis of them, may be traced continuously from one mitosis to the next, and if this is true in some cases, the failure to observe it in others does not prove that no continuity exists, but only that the conditions are unsuitable for detecting it. One of the first instances of apparent continuity to be observed was described by BovERI in the early segmentation stages of the egg of Ascaris. In A. megalocephala bivalens there are four long chromosomes with thickened ends in the equatorial plate of the first segmentation division. They divide longitudinally, so that four similar chromosomes travel to each pole and are included in the nuclei of the two-cell stage. Their arrangement in the equatorial plate varies in different eggs, as is shown in Textfig. 24. When the daughter nuclei are formed, projections of the nuclei membrane are seen, corresponding with the projecting ends of the chromosomes, and although in the resting stage no trace can be seen of the chromosomes as such, when the next nuclear division approaches the chromosomes can be seen forming in the nucleus with their ends in the projections that have persisted since the preceding telophase. The impression gained from a study of such nuclei is that although the chromosomes are invisible in the resting stage, they, or their frame-work, have been there all the time, and that they become visible in the prophase of one mitosis in the exact position in which they disappeared in the telophase of the one before.

In the plant Carex aquatilis Stout has been able to observe the chromosomes continuously from one mitosis to the next through the whole of the resting stage. The only period in which they are not recognisable is in the spireme and 
synizesis stages of the maturation of the pollen grains, when they become "spun out" into long threads that are not separable as individuals. And several investigators, in attempting carefully to follow the chromosomes from one division to the next have thrown important light on the nature of the nuclear network in the so-called resting stage. The work of Bolles LeE (1912) on the development of the pollen-grains of the plant Paris quadrifolia, and that of WiLson (I9I2) and others on the spermatogonia of several insects, may be taken as examples. Bolles LeE finds that at the close of the second maturation division of the pollen grains the chromosomes first become vacuolated and then gradually elongate into spiral threads, and that with sufficient magnification the spirals are still recognisable in the resting nucleus. The apparent reticulum of the resting nucleus is in fact a number of spiral threads, to some extent intertwined, but evidently retaining their individuality and each derived from a single compact chromosome of the preceding anaphase stage. In plants there is a nuclear division in the pollen grain after the second maturation division, and when this approaches, the fine spirals are seen to contract and thicken and to become vacuolated as they were in the preceding telophase. The vacuoles form a row along the centre of the chromosome, and mark the line along which the longitudinal division will take place. After the division is completed, the same process of forming elongated spirals can again be seen, and in the two resting nuclei of the mature pollen grain, the structure which on superficial examination appears as a reticulum is found when examined carefully to consist of these fine spiral threads.

Kristine Bonnevie has drawn similar, but notidentical, conclusions from her work on Ascaris, Allium (the Onion) and Amphiuma. She finds that within the chromosomes of 
the telophase a spiral chromatinic thread appears; the outer part of the chromosome disintegrates, and the spiral thread elongates and sends out branches which join those of neighbouring threads, so forming the nuclear reticulum. In the prophase of the next division the anastomoses are withdrawn, and the spirals contract to form the chromosomes. She points out that this means that the chromosomes of one

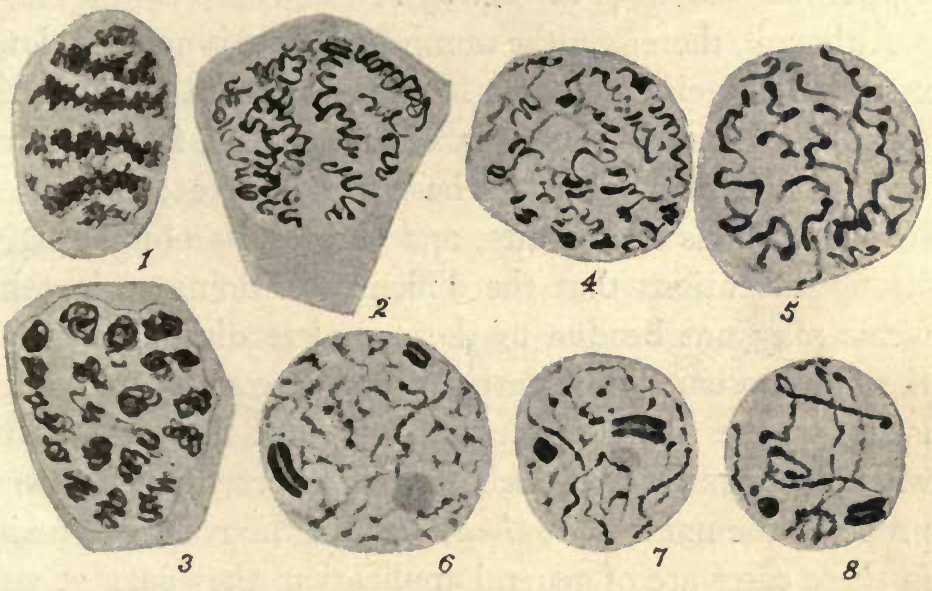

Fig. 25. Chromosomes emerging from nuclear network in Hemiptera, from WILson (1909 b). I-5, Spermatogonial prophases of Pbrynotettix. r, Reticular nucleus with irregular chromatin masses ; 2, 3, these masses becoming resolved into spirals ; 2 , side view, 3 , polar view ; 4,5 , spiral threads becoming shortened and thickened; $6-8$, emergence of elongated chromosomes from reticulum of spermatocyte of Lygaeus; the thick double rods are the $X$ - and $\gamma$-chromosomes.

division are not identical with those of the telophase of the preceding, but are formed endogenously within them.

In the spermatogonia of various insects, especially certain Hemiptera and grasshoppers, and also in other forms, a somewhat analogous condition has been described. In the resting nuclei the chromatin is distributed in masses, the number of which is probably the same as that of the chromosomes in the preceding division. When the transition to the 
leptotene stage of the spermatocyte approaches, each of these masses becomes resolved into a fine spiral filament; at first the spirals are distinct, but as they elongate they become intertwined and soon give rise to a reticular appearance of the whole nucleus. The threads of this apparent reticulum then undergo a synaptic contraction, from which emerge the pachytene threads that contract to form the spermatocyte chromosomes (Text-fig. 25).

Although, therefore, the number of cases is not very large in which the chromosomes have been traced through the reticular or resting condition of the nucleus from one mitosis to the next, this has been done quite definitely in several animals and plants, and the results obtained give strong indications that the difficulty of doing so in other forms may not be due to the complete disappearance of the chromosomes, but rather to their complicated interlacing in the so-called reticular stage of the nucleus, for which Bolles LEe, on the basis of his observations on Paris, proposes the name spirophase. And if the results obtained in these cases are of general application, they suggest very clearly that the individuality of the chromosomes does persist in the resting nucleus. It is probable, however, that the elongated filaments of the resting ("spirophase") stage are not precisely equivalent to the chromosomes of the mitotic figure, for as has been described in an earlier chapter, not only does the absolute amount of chromatin in the nucleus, as judged by its staining capacity, undergo considerable fluctuations at different stages, but there is also strong evidence that, in some cases at least, substance is withdrawn from the nucleolus and converted into the chromatin of the mitotic chromosomes. Several instances have also been referred to of the emission of chromatin from the nucleus, into the cytoplasm. It would seem, therefore, that what 
persists from one mitosis to the next is not the precise masses of chromatin which constitute mitotic chromosomes, or at least make up the greater part of their substance, but rather a framework or basis in which a greater or less amount of chromatin is concentrated at different times. Here again the analogy - though it is only an analogy - of independent symbiotic organisms may be helpful. If such organisms, for example Zooxanthellae, contained chlorophyll, the amount of it might vary from time to time, giving rise to corresponding variation in their visibility, but the organisms would nevertheless persist as individuals even though at times they were only distinguishable with difficulty in consequence of their temporary poverty in chlorophyll. The chromosomes, in fact, must not be imagined as simple masses of chromatin, but as organised bodies which contain a greater or less amount of chromatin at different times, and it is the bodies themselves that must be regarded as persistent individuals, and not merely the chromatin which by its staining capacity makes them especially visible during mitosis.

(3) The recognisable and definite differences in size and shape of the chromosomes.

Under the heading of the definite number of chromosomes no explicit reference was made to the constant differences of size and shape which characterise the chromosomes of many animals, although this was implicitly assumed in referring to Wilson's observations in Metapodius. In quite a large number of animals, especially in the Orthoptera, Hemiptera and Diptera among insects, and in Echinoderms, some or all of the chromosomes are constantly recognisable by their size, by their shape in the anaphase of mitosis, and frequently also by the shape of the bivalents in the first maturation (heterotype) division of the germ-cells. In 
species with relatively few chromosomes, such as grasshoppers and a number of Diptera, each chromosome is easily recognisable in the equatorial plate of any division, and MEEK has shown that in the spermatogonial and spermatocyte divisions each chromosome has constant dimensions. In division-figures of nuclei which have the diploid number there are of course two of each kind (excluding the sexchromosomes, which may be unpaired), and in the Diptera the two homologous chromosomes commonly lie side by side on the spindle in all somatic mitoses. When the maturation period of the germ-cells approaches, these pairs of homologous chromosomes unite to form bivalents (gemini), and separate to opposite poles in the reducing division, with the result that each germ-cell contains a single complement including one of each kind. The individuality of the sexchromosomes (heterotropic or idio-chromosomes) is of course a still more striking example of the same thing-the constant appearance of a definite chromosome of characteristic behaviour in every cell of the individual. As their behaviour has been described in Chapter XI, no further reference to them is needed here.

The argument for chromosome individuality from the definiteness and permanence of appearance and behaviour of particular chromosomes in ordinary individuals of many species is strongly reinforced by the examination of hybrids. It has been observed in a number of cases that when a hybrid is made between two species in which the chromosomes are recognisably different in size, both kinds of chromosomes retain their characters in the hybrid. The example already referred to in Chapter VI will suffice to make this clear. The moth Biston birtarius has in its diploid cells 28 chromosomes, of which 24 are moderately large and four quite small. The nearly related moth Nyssia zonaria 
has 56 chromosomes in the mitoses of the maturation divisions, and, as nearly as can be counted, II 2 in its diploid nuclei; all are very small, but four (two in reduced nuclei) somewhat larger than the rest. The mature germ-cells of birtarius have thus 14 (twelve large and two small); those of zonaria have 56 (two of moderate size and 54 small). The hybrid has in its spermatogonia, as nearly as it is possible to count, 70 chromosomes, of which 14 are noticeably larger than the remainder. This by itself would indicate that the chromosomes of the two species retain their individuality in the hybrid, but the phenomena seen in the spermatocyte divisions confirm this conclusion in a very striking way. As already described (p. 87), since there is only a single set from each parent species, and the chromosomes of one species are not homologous with those of the other, very few of the chromosomes are able to find mates in the prophase of the maturation divisions, and in consequence the spermatocyte equatorial plates have only a few chromosomes less than those of the spermatogonia, instead of half the number. If the number and size of the chromosomes depended simply on an inherent tendency of the species, causing the chromatin to be aggregated into a constant number of masses at mitosis, these facts are inexplicable, but on the individuality hypothesis they are precisely what would be expected.

(4) The different physiological values of distinct chromosomes.

An argument based on the physiological function of the chromosomes is necessarily speculative, since at present their significance in this respect can only be inferred, or, as some would say, guessed at. It seems clear, however, that the idio-chromosomes have a definite function in connection with sex-determination, and in the next chapter the possi- 
bility of the chromosomes being connected with the transmission of hereditary characters will be considered. On the supposition that the chromosomes play an important part in the life of the cell, and therefore of the development of an embryo, BoverI has based an ingenious argument in favour of the belief that different chromosomes have different functions in this respect. When in Echinoderms two spermatozoa enter an egg, as may happen if the egg is treated
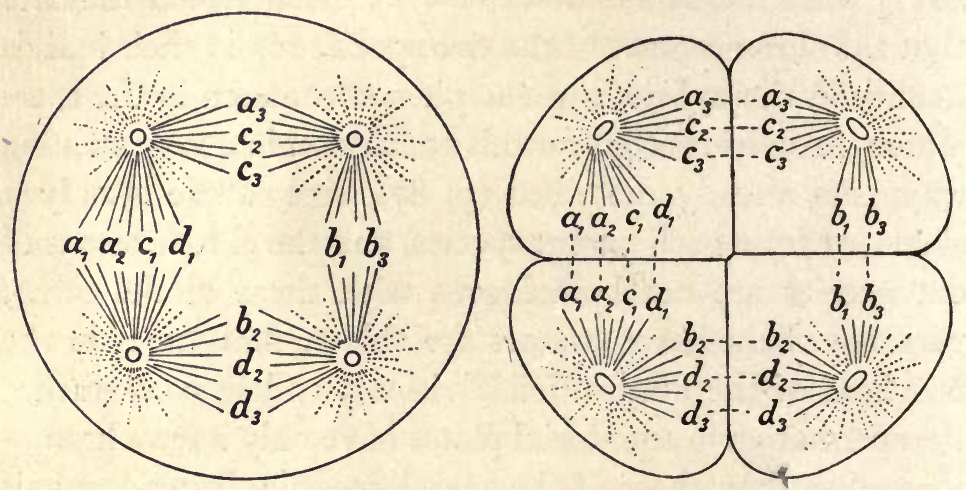

FIG. 26. Diagram of the distribution of chromosomes on a quadripolar spindle, from BOVERI ( 1903 a).

with an anaesthetic before fertilisation, a quadripolar spindle may result in the first segmentation division. Each spermatozoon gives rise to two centrosomes, and these four centrosomes become connected by four spindles, with the result that the egg divides simultaneously into four blastomeres. Since there are, at the beginning, three nuclei (egg nucleus and two sperm nuclei) each with the reduced number $(n)$ of chromosomes, there are in all $3 n$ chromosomes distributed in the equatorial plates of four spindles. And since each chromosome divides and its halves pass to the poles of its spindle, there will be altogether $6 n$ chromosomes distributed among the four daughter nuclei, or an average 
of $\frac{6 n}{4}=\frac{3^{n}}{2}$, in each nucleus. In a normally fertilised egg there are of course $2 n$ in each nucleus of the segmenting egg, but the phenomena of artificial parthenogenesis show that half this number $(n)$ is sufficient to allow of normal development. If, therefore, the chromosomes were functionally all alike, every cell that receives as many as $n$ chromosomes should be capable of normal development. If, however, the chromosomes differ from one another in function, it is unlikely that a complete set will be included in each of the four nuclei resulting from the quadripolar spindle of a dispermic egg, if the three sets of chromosomes are distributed on the four spindles at random. In the accompanying diagram, reproduced from BovERI, the number $n$ is represented for convenience as four (in Echinoids it is really about eighteen), and the chromosomes from the three germ-nuclei are represented by $a_{1}, b_{1}, c_{1}, d_{1}, a_{2}, b_{2}, c_{2}, d_{2}, a_{3}, b_{3}, c_{3}, d_{3}$. If, now, the chromosomes are distributed on the spindles in some such way as in the diagram, only one of the four daughter nuclei (the left lower) will receive all four kinds of chromosomes $(a, b, c, d)$; the left upper has no $b$, the right upper no $d$, and the right lower neither $a$ nor $c$. This imaginary example is probably an extreme one, but when the full (reduced) complement is eighteen, it is quite likely that one or more of the nuclei will lack some members of the complete series, although each nucleus contains not less than $n$ chromosomes in all. If, then, simply $n$ chromosomes were required for normal development, each of the four cells should be able to continue its development in the typical way, but if the chromosomes differ from one another in function, it might be expected that the parts of the larva derived from one or more of the first four blastomeres should sooner or later show abnormalities. And this is what is actually found, as 
Boveri points out. Some larvae from dispermic eggs are completely normal; others develop normally up to the gastrula stage, or even later, but lack the skeleton in one half or one quarter of the larva in the pluteus stage; while others begin to show abnormalities in the blastula and fail to become gastrulae. Furthermore, if the blastomeres of a normal four-cell embryo are shaken apart, each produces a complete small larva, but the separated blastomeres of a dispermic egg are rarely able to do this, although some proceed very much further in development than others. Now the only difference between the four cells of a dispermic egg is in the chromosomes; if abnormalities are induced in the cytoplasm they must be common to all four cells. Since, therefore, these cells differ widely from one another in their powers of development, it must be because some have received chromosomes that are absent from others.

This kind of argument cannot be regarded as a conclusive proof of the functional individuality of chromosomes; it rather assumes that individuality, and shows that the results observed are easily explicable on that assumption, but are hard to account for otherwise. A weak point in the argument is that in polyspermic eggs of the frog, as described by BRACHET (I9I7), the early development may be normal, and yet the larvae die after hatching, although the conditions are such as to exclude Boveri's interpretation. In poly= spermic frog's eggs one spermatozoon conjugates with the egg-nucleus, while the others form independent nuclei in connection with each of which a spindle develops, and the egg divides into as many blastomeres as there were spermatozoa. In the resulting embryo, therefore, some nuclei contain the diploid, others the haploid number of chromosomes, but every cell contains at least the whole haploid series, and yet, when the embryo becomes a larva, it is not able to 
survive. BRACHET ascribes this non-viability to incompatibility between the tissues arising from such different cells, and if this is so in the frog, some similar condition may perhaps also exist in those parts of the dispermic Echinoid larva which fail to develop normally.

In conclusion, although all the four groups of arguments given above in favour of the individuality theory refer to the chromosomes as complete entities, it is important to remember that they are in all probability divisible into smaller units, and that these units, supposed to be arranged in a definite order along the length of a chromosome, probably have an individuality more fundamental than the chromosome as a whole. That this is so is strongly suggested by such cases as that of $A$ scaris, and is a necessary assumption for the hypotheses regarding the function of the chromosomes in heredity that will be considered in the next chapter. Not infrequently, especially in the spireme stage of the nucleus, the chromosomes can be seen to be made up of a series of granules, like beads on a string, and with the support of instances like the compound chromosomes of the germ-track of $A$ scaris, it seems a not unreasonable extension of the individuality theory to regard these granules ("microsomes") as the fundamental units, and to modify the theory in the way suggested in the introductory portion of this chapter. 


\section{CHAPTER XIV}

\section{The Mechanism of Hereditary Transmission}

Dinecialiy since Weismann brought forward the first C outline of his theory of heredity in 1885 , one of the chief motives and objects of cytological investigation has been the discovery of the means by which inherited characters are transmitted from parent to offspring. We know that in general the father and mother contribute equally to the hereditary features of their children, and yet in Man the whole of this almost infinitely complex web of family likeness is transmitted, on the father's side, by a spermatozoon about one five-hundredth of an inch in length ${ }^{1}$. If the facts of heredity were not so familiar, they would be regarded as the most wonderful thing in nature, and it is not surprising that one of the chief aims of cytologists has been to find some explanation of their mechanism.

WEISMAN N's famous theory of the germ plasm was largely instrumental in leading to more thorough investigations of the behaviour of the chromosomes, for according to his scheme these bodies bore the "ids" or determinants which were the basis of hereditary transmission. It is not intended here to discuss these older hypotheses; much of Weismann's complicated theory is already disproved and almost forgotten, but it is impossible to consider more recent ideas on the subject without referring to the fact that he not only provided the stimulus which has led to such brilliant results in the last few years, but also first gave sound reasons for

1 A human spermatozoon is approximately $05 \mathrm{~mm}$., or about $\frac{1}{500}$ inch, in length, and of this length fully nine-tenths is tail. 
the belief, which is daily gaining ground, that the chromosomes have a preponderant share in the transmission of hereditary characters.

The arguments for ascribing this leading part to the chromosomes in hereditary transmission, like those for the individuality of the chromosomes themselves, are of several distinct kinds, some direct and some purely inferential. They may be classed at the outset into two groups, firstly, those which indicate that the nucleus rather than the cytoplasm is the more important factor, and secondly those which point to the chromosomes rather than any other nuclear constituent as being the "bearers of hereditary characters." The main lines of argument will be dealt with in this order.

One obvious reason for supposing that the nucleus plays the chief part in the transmission of inherited characters is that the spermatozoon consists almost entirely of nucleus, middle-piece, and tail, and of these the tail in some forms is thrown off as the spermatozoon enters the egg, and the middle-piece seems to give rise to little beside the centrosomes. Further, the egg and spermatozoon are equivalent as regards the transmission of most characters, and the nuclei are the only parts of them which are alike; if the cytoplasm took any preponderant share, it is hardly possible to believe that the maternal characters would not be more strongly developed in the offspring than the paternal. There are also several kinds of experimental evidence pointing to the same conclusion. It is known that non-nucleated fragments of Sea-urchin eggs can be fertilised by a spermatozoon and give rise to larvae which show the normal characters of the species. BoverI $(1895,1903 b)$ attempted to use this fact to test the hypothesis that the nucleus bears the determiners for hereditary characters by fertilising non-nucleated 
fragments of the eggs of one species (Spbaerechinus granularis) by the sperm of another (Echinus microtuberculatus). He shook up the eggs in water in a tube till they were broken up, and picked out under the microscope non-nucleated fragments to which Echinus sperm was added. Some of these fragments segmented and developed into larvae which in their skeletal characters at least were of the pure Ecbinus type. If there are no sources of error, this experiment proves that some characters at least are determined purely by the nucleus. Objection, however, has been taken to it on various grounds-chiefly that the ordinary hybrids of these species, made from nucleated eggs, may at times have the Ecbinus type of skeleton. Boveri, however, in his control experiments never got hybrid larvae showing no Spbaerecbinus characters, and the experiment may probably be taken as proving that the skeletal characters of Echinoid larvae are determined by factors contained in the nucleus.

Additional evidence is provided by the experiments of Herbst. He treated the eggs of Sphaerechinus with a fatty acid that induces artificial parthenogenesis, and then, before segmentation began, fertilised them with sperm of Strongylocentrotus lividus. By this procedure the nucleus in some eggs was caused to divide before the sperm nucleus was ready to conjugate with it, and the sperm nucleus was carried into one of the first two blastomeres, and there conjugated with its nucleus. As a result, one of the first two blastomeres contained a nucleus of purely maternal origin, and containing the reduced number of chromosomes, while the other was hybrid, and contained both paternal and. maternal chromosomes. Among the plutei reared from eggs treated in this way, some were found in which one half of the body had purely maternal skeletal characters, while the other half. had the characters commonly found in 
hybrids, and it was further observed that the nuclei on the purely maternal side were much smaller than those on the side with the hybrid skeleton. Boveri has shown that in Echinoids the size of the nucleus is generally proportional to the number of chromosomes, and it may therefore be assumed that the half of the pluteus with maternal skeletal characters and small nuclei is derived from a blastomere
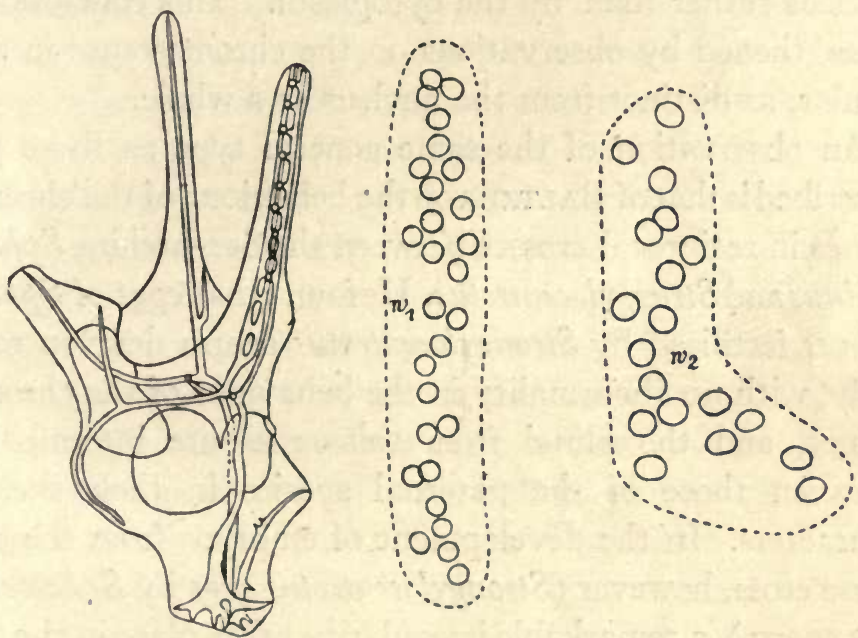

FIG. 27. A "partially thelykaryotic" pluteus from the cross Sphaerechinus $q \times$ Strongylocentrotus $\delta$. The left side of the pluteus has the skeleton of the paternal (Strongylocentrotus) type; on the right the skeleton is intermediate but more nearly resembling Spbaerechinus. $w_{1}$ represents small nuclei from the ciliated band of the left (paternal) side; $w_{2}$ corresponding larger nuclei of the right side. (From HERBST, I 907.)

with a purely maternal nucleus and the reduced number of chromosomes, and the hybrid half from the other blastomere in which the nucleus conjugated with the sperm nucleus. The only objection to this conclusion is that larvae with asymmetrical skeletons are common in the hybrids of these species, but if Herbst's "partially thelykaryotic" larvae were simply examples of this asymmetry, the difference in

D. C. 
size of the nuclei on the two sides is quite inexplicable. Hybrids also practically never show the maternal (Sphaerecbinus) characters developed to the extent to which Hersst found them in the maternal halves of his larvae.

Experiments such as those described, which by no means exhaust all the evidence available, give strong indications that the factors for hereditary characters are borne by the nucleus rather than by the cytoplasm. This conclusion is strengthened by observations on the chromosomes in particular, as distinct from the nucleus as a whole.

An observation of the same general type as those just described is that of BALTZER on the behaviour of the chromosomes in reciprocal crosses between the Sea-urchins Sphaerechinus and Strongylocentrotus. He found that eggs of Sphaerchinus fertilised by Strongylocentrotus sperm develop regularly, with no abnormality in the behaviour of the chromosomes, and the plutei from this cross are intermediate between those of the paternal species in their skeletal characters. In the development of embryos from the converse cross, however (Strongylocentrotus eggs by Sphaerechinus sperm), a remarkable irregularity takes place in the first and second segmentation divisions of the cross-fertilised egg. In these divisions altogether fifteen or sixteen chromosomes are omitted from the division spindles. They split in the equatorial plate of the first division, but fail to divide completely, and some are left behind on the spindle in the anaphase stage, while others are carried with the normal chromosomes to one or other daughter nucleus. In the second division, those which have been included in the nuclei reappear as split rods, and are left out when the four daughter nuclei are formed. The haploid numbers of the parent species are twenty for Sphaerechinus and eighteen for Strongylocentrotus, and after the elimination of the abnormal 
chromosomes twenty-one or twenty-two chromosomes are found in the subsequent mitoses. BALTZE R believes that the eliminated chromosomes are all paternal (from Sphaerechinus), chiefly on the grounds (I) that Sphaerechinus has two long straight chromosomes and Strongylocentrotus a long hooked one, and that in the hybrid embryos the straight chromosomes are never present while the hooked one always is; and (2) in a quadripolar spindle derived from a dispermic egg there are only a few (about four) more chromosomes behaving normally than in an ordinary mitosis, while if the paternal chromosomes behaved normally there would be twenty extra. Nearly all the embryos become abnormal in the gastrula stage, and die off, but in the very rare cases in which plutei have been reared these were of the maternal type. If the eliminated chromosomes are paternal, their absence would thus account for the lack of paternal characters in the hybrid, while in the converse cross no chromosomes are eliminated and the hybrid pluteus shows the characters of both parents. Similar elimination of chromosomes has been observed by Miss Pinney in Teleostean hybrids.

Further evidence in favour of the hypothesis that the chromosomes are of especial importance in determining inferited characters has been obtained from plants. In Oenothera (Evening Primrose) at least two species have a variety known as lata, in which the leaves are broader than in the type form, and these lata varieties have one more chromosome than the normal, the typical number being fourteen while lata has fifteen. Since one chromosome is unpaired, various irregularities arise in the maturation divisions of the germ-cells; the unpaired chromosome may go over undivided to one pole in the heterotype division, and divide equationally in the homotype, so giving equal 
numbers of gametes which have it and are without it; or it may be omitted from the daughter nuclei altogether, or in some cases it may divide in the first and pass to one pole without division in the second. In consequence of this variable behaviour less than half the germ-cells possess the odd chromosome, the number varying in different plants, and correspondingly it is found that lata parents crossed with the type form give a variable percentage of lata offspring, ranging from 4 to 45 per cent. Gates, to whom our knowledge of the facts is chiefly due, has occasionally seen abnormalities in the maturation of the pollen cells in the type form of Oenothera by which both members of a pair of chromosomes go to one pole in the heterotype division, and he supposes that the fifteen-chromosome (lata) races arise in this way. It is not known whether the extra chromosome is always homologous in different lata races, or whether the duplication of any chromosome produces a lata form; since there is another variety (semilata) that also has fifteen chromosomes, it is possible that the duplication of one chromosome produces lata and of a different one semilata.

Another variety of Oenothera that is correlated with a difference in the chromosome number is the form gigas, which has twice the normal number, that is to say, it is "tetraploid" instead of diploid, and has 28 chromosomes in its somatic cells. In this form also there are irregularities in the maturation divisions, leading to frequent sterility. Another somewhat similar case of a tetraploid variety in Primula will be described after the possible relation between chromosomes and Mendelian characters has been considered.

In the examples given above there is more or less direct evidence, even though it is not always as satisfactory as might be wished, of a connection between one or more 
chromosomes and the appearance of definite somatic characters. This evidence is greatly strengthened by arguments of a more indirect kind, based on the extraordinary similarity between the known behaviour of the chromosomes in the maturation divisions of the germ-cells and the manner of transmission of Mendelian characters. In the chapters on spermatogenesis and oogenesis, it has been seen that the behaviour of the chromosomes can be summarised as follows. Since the chromosomes of the diploid nucleus before maturation are in pairs, the members of each pair being descended from the corresponding chromosomes introduced into the fertilised egg from the two parents, they may be represented as $A, B, C, D \ldots$ derived from one parent and $a, b, c, d \ldots$ derived from the other. In the maturation divisions $A$ pairs with $a, B$ with $b$, and so on, and the members of each pair pass into different germ-cells. There is no reason, however, for supposing that chromosomes of different pairs, but derived from the same parent, have any tendency to go together; that is to say, $A$ may equally often be associated with $B$ or with $b$, and with $C$ or with $c$. The mature germcells may therefore contain $A, B, C, D \ldots, A, b, C, D \ldots$, $A, b, c, d \ldots, a, B, c, D \ldots$ or any other combinations, so long as no two members of one pair are included in one cell. Now in the segregation of independent Mendelian characters precisely this kind of distribution is found; the characters occur in pairs, which are said to be allelomorpbic with each other, and the two members of an allelomorphic pair are never transmitted by the same germ-cell. If two varieties are crossed, differing in three pairs of characters which may be called $M, N, O$ and $m, n, o$, the heterozygote will contain the "factors" for all six, but its germ-cells will bear, on the average in equal numbers, those for all combinations in which neither $\mathrm{Mm}, \mathrm{Nn}, \mathrm{O}_{0}$ are included. Or, expressed 
differently, the germ-cells will bear the factors $M N O, M N o$, $M n o, m N O$ and so on. It is evident, as Sutton pointed out in 1903 , that the correspondence between the distribution in the germ-cells of the factors for Mendelian characters and that of the paternal and maternal chromosomes is so exact as to make it hardly possible to doubt the existence of a causal connection between them.

In matings in which both parents belong to the same form the homologous chromosomes $A$ and $a$ etc. would of course be alike, and would bear identical Mendelian factors. When, however, a mutation takes place, it must be supposed to arise by a change in, or loss of, a factor in some part of one chromosome. This may be represented by saying that factor $M$ is changed to $m$. If, then, chromosome $a$ now bears factor $m$ instead of $M$, there will be Mendelian segregation between the factor $M$ borne by chromosome $A$ and factor $m$ borne by $a$. This may perhaps be made clearer by a specific instance that will be discussed more fully later in this chapter. In the fly Drosopbila there are reasons for believing that the factor for the red eye, which is characteristic of the normal fly, is borne by the $X$ (sex) chromosome. A mutant form of the species has white eyes, and the red and white factors are allelomorphic. It is therefore supposed that the factor for white eye is due to a change in, or loss of, that unit in the $X$ chromosome which in the normal fly determines the red-eye character. In the heterozygous (hybrid) fly produced by mating red with white eyed, one $X$ chromosome bears the factor for red, the other for white eyes, and since these chromosomes separate from each other at the maturation of the egg, the red and white factors must segregate in the Mendelian manner.

There are, however, several difficulties in the way of accepting the hypothesis that the segregation of the chromo- 
somes in gametogenesis is the mechanism by which the segregation of Mendelian characters is brought about. These difficulties will be discussed below, and it will be seen that most of them are not insuperable, but first it may be advisable to mention two pieces of direct evidence in favour of the hypothesis.

FEDERLey has shown that when the moth Pygaera curtula is crossed with $P$. anachoreta, the chromosomes behave in the gametogenesis of the hybrid much in the same way as in the cross, already referred to, between Biston birtarius and Nyssia zonaria. The reduced number in curtula is 29 , in anachoreta 30 ; the spermatogonia of the hybrid have 59, but in the spermatocytes very few chromosomes pair; they. divide equationally in both divisions, with the result that the mature germ-cells contain about 59. If, now, the hybrid is mated back with pure anachoreta, a secondary hybrid with nearly 89 chromosomes in its spermatogonia is produced. In the spermatocytes of the secondary hybrid the two sets of anachoreta chromosomes pair together, so that on the spindle of the first spermatocytes there are about 30 large (bivalent) chromosomes derived from anachoreta and about 29 univalents derived from curtula. The mature germ-cells of the secondary hybrid therefore again contain nearly 59 chromosomes. Corresponding with these cytological facts FEDERLEY found that in the primary hybrid some characters of anachoreta and others of curtula were dominant, while in other features the hybrid was intermediate between the parents. When the hybrid was mated back with pure anachoreta, no segregation of the moth characters took place; all the secondary hybrids had the same characters as the primary. In one larval feature, however, segregation took place. Now since all the germ-cells of the secondary hybrid contain both curtula and anachoreta chromosomes, 
as in the primary hybrid, if the chromosomes bear the "factors" for Mendelian characters, it is to be expected that no segregation will occur. But FEDERLEY observed that in the spermatocytes of the hybrids, at least in some cases, one or two chromosomes paired and separated, and he suggests that the segregation of the larval character is due to its being borne by one of these chromosomes.

The second example of direct evidence in favour of the hypothesis is that of the late R. P. GREGory on a tetraploid race of Primula sinensis. In this race there is twice the normal number of chromosomes; that is to say, there are four instead of two of each kind. The race is fertile when two tetraploid plants are crossed together; each germ-cell contains the diploid number of chromosomes, and the zygote again is tetraploid. If, now, the chromosomes determine the segregation of Mendelian characters, such a tetraploid race should show a new type of segregation, for in the zygote there would be four chromosomes of each kind, and in the germ-cell two. Suppose a plant to receive the character $A$ from one parent, and its allelomorph $a$ from the other, then, since each chromosome is duplicated, the heterozygote would be $A A a a$, and instead of producing two kinds of germcells, $A$ - and $a$-bearing, it would produce three, $A A, A a$, and $a a$, in the ratio of $\mathbf{I}: 2: \mathbf{I}$. Such a plant self-fertilised would give fifteen offspring showing the dominant $A$ character to one showing the recessive $a$. This is exactly what GrEgory found; instead of getting a $3:$ I ratio when the heterozygote is self-fertilised, he got ratios approaching $15: \mathrm{I}$, and similarly, when the heterozygote was crossed back with the pure recessive, instead of a I : I ratio he got $3: \mathbf{I}$. At first sight this appears a convincing demonstration that the Mendelian characters of Primula sinensis are borne by the chromosomes, or at least that their segregation is determined 
by them, but GREGory points out that this conclusion does not necessarily follow, very probable though it may be. He writes "Although the fact that the duplication of the chromosomes has been accompanied by a duplication of the series of factors may seem at first sight to suggest a definite connection between chromosomes and factors, yet, on the other hand, the tetraploid number of chromosomes may be nothing more than an index of the quadruple nature of the cell as a whole. The case is, in fact, exactly analogous to the ordinary zygotic cell, which has $2 x$ chromosomes and in which each factor is represented twice."

Additional evidence, some of it of very great importance, will be given below in support of the hypothesis that the behaviour of the chromosomes in the maturation of the germ-cells is responsible for the segregation of Mendelian characters, but first it will be advisable to consider some of the difficulties by which the hypothesis is faced. These may be grouped in two classes, first those raised by cytologists, and second those arising from the facts of genetics.

The cytological objections that have been raised against the hypothesis are of several kinds. Some observers deny the existence of any such individuality in the chromosomes as is required by the hypothesis. If the individuality theory is false, the hypothesis that the chromosomes determine the segregation of Mendelian characters necessarily falls with it, but the facts and arguments in favour of the existence of some sort of individuality have been considered in the preceding chapter, and no further discussion of them is required here. A second objection that has been raised is that although the main facts already described are admitted, it is maintained that when the chromosomes come together in pairs before the maturation divisions, they do not merely come into contact and then separate as complete units in 
the heterotype division, but fuse so intimately that the chromosomes after separation cannot be regarded as identical with those which paired in the synaptic stages ${ }^{1}$. Other observers, again, maintain that after the chromosomes have come together in pairs they never separate again, and that in both the maturation divisions they split longitudinally, with the result that each chromosome of the mature germcells contains parts of the two chromosomes which paired in synapsis ${ }^{2}$. Both these latter arguments would be fatal to the hypothesis under consideration if they were substantiated, for each maintains that the chromosomes of the mature germ-cells contain a mixture of paternal and maternal elements, and the essence of Mendelian segregation is that the characters borne by the germ-cells are pure and not mixed. The evidence, however, on which these observers rely is negative; in their material they cannot see the separation of complete chromosomes and so they deny its existence. But if it is visible in material derived from other species which are possibly better adapted for the study of these stages, it seems more probable that the failure of some observers to find it is due rather to the unsuitable material on which they have worked than to the real absence of a true "reduction division ${ }^{3}$."

The chief arguments that have been brought against the hypothesis from the standpoint of genetics are, first, the existence of segregation of characters elsewhere than in the germ-cells, and, second, the fact that there are many more Mendelian characters than chromosomes in at least several species. The evidence for segregation of inherited characters

1 e.g. Bonnevie, 1908.

2 e.g. MAtSCHECK.

3 Since in some cases the chromosomes appear to fuse more or less completely in synapsis, it has been suggested by several writers that when this happens, "blended" inheritance results, while when they pair and separate without fusion the factors borne by them segregate according to MENDEL's Law. 
at stages other than the maturation of the germ-cells is provided chiefly by plants. "Bud-variation" is a not uncommon phenomenon in many plants, and the character which distinguishes the varying shoot from the rest of the plant may be one that is known to follow Mendel's law when the two forms are crossed together. In such a case it is clear that segregation has taken place between the main plant and the varying bud. This, however, does not invalidate the chromosome hypothesis, for the variation of the bud may have been due to an irregular division in the cell from which the bud was derived, by which, perhaps, one chromosome, or part of a chromosome, was omitted from the daughtercell that gave rise to the bud. Less easy to explain are those cases in which one part of a plant constantly bears Mendelian characters that are lacking in another. In those forms of the Stock (Matthiola), for example, which are heterozygous for the factor for doubleness, Miss SAUnders has shown that all the pollen grains bear the recessive double character, while of the ovules some bear the double and some the single factor. In this form, therefore, the dominant factor for singleness is eliminated at some stage of the development of the pollen before the maturation divisions take place. Another remarkable case is that described by Bateson and Miss Pellew in peas. Certain peas throw a small proportion of abnormal offspring known as "rogues," and when these rogues are crossed with the type the young plants have the type form, but as they grow up they become rogues and produce only rogue offspring. As the heterozygous plant grows, the type characters (to quote Bateson and Pellew) "are in some way used up and cut out of the germ-lineage in the early stages of the somatic development." If the chromosome hypothesis be true, a chromosome, or part of one, must apparently be omitted from 
the nuclei at some stage, or the chromosome must undergo a qualitative change during the development of the plant, and it should be noticed that in the "rogue" pea the change of type during the growth of the plant is a constant phenomenon in some varieties, and not the apparently irregular and accidental event to which bud variation is commonly ascribed. Until cases such as these have received an adequate explanation, the hypothesis that the segregation of Mendelian characters takes place by the separation of chromosomes at the maturation divisions of the germ-cells cannot be regarded as fully established.

A further difficulty of somewhat the same sort arises, especially in plants, from the divergence from expectation of the numbers of germ-cells bearing the alternative characters of a pair. If in the production of germ-cells by a heterozygote the segregation of alternative characters takes place at the maturation divisions, there should always be equal numbers bearing each of the two characters. When the numbers from several or many families are added together, this is generally true, except when disturbing causes, such as differential mortality, intervene, but especially in plants it is often not true of individual families. Quite often in some families there is a constant excess of one character and deficiency of the other, as compared with expectation, and this has led some investigators to believe that the segregation occurs at an earlier stage, and that in some individuals an excess of cells bearing one character, in others of cells bearing the other, is produced by unequal multiplication of the two kinds of cells in the germinal tissue. Such multiplication could only occur before the maturation divisions, for in the higher plants no multiplication of germ-cells occurs after it. Irregularities of this sort, while not unknown in animals, are less frequent in them than in plants. 
The second chief objection brought on genetic grounds against the chromosome hypothesis is that there may be more Mendelian characters in a species than there are chromosomes, and that, nevertheless, these characters appear to segregate independently of one another ${ }^{1}$. The answer to this objection leads up to a most interesting extension of the hypothesis, due to Prof. Morgan of New York, and provides an important argument in favour of the hypothesis as a whole.

If there are more Mendelian characters than chromosomes, and if the chromosomes in some way bear the determiners or "factors" for these characters, one of two things must follow. Either the chromosomes must be made up of smaller units which are to some extent interchangeable, or, if the chromosomes behave as indivisible units, all the factors for characters that are borne by one chromosome must be associated with one another in inheritance. In the earlier cases to be discovered, in which the pairs of Mendelian characters were more numerous than the haploid number of chromosomes, no such association of the characters was known, and several suggestions were made with regard to the manner in which the factors were borne by the chromosomes. One was that the true units were the microsomes, or small particles of which the leptotene threads are composed, and that when the chromosomes unite in pairs these units might be interchanged. Another, based on Fick's "Manoeuvre-hypothesis," was that in the resting stage of the nucleus the constituent elements of the chromosomes became separated, but came together in the same formation

1 It is pointed out by WINGE (1919), however, that no case is known in which the number of simultaneously and independently segregating pairs of characters existing together in one individual exceeds the haploid chromosome number of the species. 
for each mitosis. Since, however, there are two chromosomes of each sort in the diploid nucleus, it was supposed that the small units of which they were composed were interchangeable, and that therefore the chromosomes of one mitosis were not necessarily identical with those of the preceding, but that the chromosomes of each homologous pair were made up from a double set of microsomes. The microsomes would be in two series, $a, b, c, d \ldots$ and $a^{\prime}, b^{\prime}, c^{\prime}, d^{\prime} \ldots$, arranged in this order, but when the mitotic chromosomes are formed from the resting nucleus $a$ is interchangeable with $a^{\prime}, b$ with $b^{\prime}$, and so on. It has been seen in the preceding chapter that there is evidence for the compound nature of chromosomes, at least in some species, but the evidence for their general individuality as complete wholes does not favour this hypothesis of the interchange of parts in the resting nucleus.

-The matter has entered on a new phase since the discovery and thorough investigation of the phenomenon known in England as gametic coupling, and in America as linkage, first described by Bateson and Punnett in the Sweet Pea, but now known in many plants and animals, and most fully investigated by Morgan and his associates in the fly Drosopbila. The essence of gametic coupling is that if varieties are crossed which differ in two or more characters, the characters that are associated in the parents tend to be associated in the gametes of the heterozygous offspring. If, for example, an individual bearing the characters $A$ and $B$ is mated with one bearing their allelomorphs $a$ and $b$, the heterozygote $A a B b$, instead of producing in equal numbers four kinds of gametes bearing $A B, A b, a B, a b$, produces great excess of $A B$ and $a b$ and relatively few $A b$ and $a B$. This "coupling" in transmission by the heterozygote of characters that were associated in the parents strongly 
suggests that the factors for the coupled characters are borne by some body which behaves in gametogenesis as a unit, and the only bodies known to behave in the way required are the chromosomes. This conclusion is greatly strengthened by the facts as they are now known in Drosopbila. In $D$. ampelopbila the haploid number of chromosomes is four, and there are four pairs, easily distinguishable from one another, in the diploid cells. Of these four, two are long ordinary chromosomes (autosomes), one is a very short autosome, and one a sex-chromosome. To the last further reference will be made later; the facts which bear particularly on the present discussion are that in heredity it is found that there are four groups of coupled characters, which may be numbered I, II, III, IV. According to Morgan and his associates, when a female bearing any two or more of the characters in group $I$ is mated with a male that lacks them, all these characters are found to be coupled in the germ-cells of the offspring, and the same is true of the characters in groups II, III, and IV, but there is no coupling between characters belonging to different groups. Furthermore, groups I, II and III each contain a considerable number of Mendelian characters, while group IV is small, and only two characters belonging to it have as yet been discovered. On the basis of these facts, Morgan supposes that the factors for characters in group $\mathrm{I}$ are borne by the sex-chromosome (the reasons for this will be given below), those of groups II and III by the two long autosomes, and of group IV by the small autosome. If no other group of coupled characters is discovered, the correspondence between the coupled groups and the chromosomes is so remarkable that it seems hardly possible to doubt the existence of a connection between them.

One very important group of facts, however, remains to be explained. Although when a parent bearing the coupled 
characters $A$ and $B$ is mated with one bearing $a$ and $b$, the majority of the gametes of the offspring also transmit $A$ and $B$ or $a$ and $b$, yet absolute coupling is very rarely, if ever, found. Nearly always some gametes bearing $A$ and $b$ or $a$ and $B$ are also produced, the relative frequency of these exceptional combinations varying greatly in different pairs. The American investigators call these exceptional combinations cross-overs, since in the combinations $A b$ and $a B, A$ and $a$ are regarded as having crossed over from their normal combinations and to have exchanged places. In respect of any pair of characters, the number of cross-overs is on the average fairly constant, ranging from less than I per cent. in some pairs of characters to as much as 40 per cent. in others. If there were 50 per cent. of cross-overs there would of course be no coupling, and the characters would be transmitted independently of each other. The relative number of crossovers is thus a measure of the intensity of the coupling, a high intensity being synonymous with a low ratio of crossovers. It is further found that when there are in a coupled group several characters $A, B, C, D \ldots$, they can be arranged in a series in such a way that the ratio of cross-overs between the extremes is approximately equal to the sum of the ratios between the characters placed next to each other; for example, if there is I per cent. of crossing over between $A$ and $B, 30$ per cent. between $B$ and $C$, and 3 per cent. between $C$ and $D$, then there will be about 3 I per cent. between $A$ and $C, 33$ per cent. between $B$ and $D$, and 34 per cent. between $A$ and $D$. These numbers are only approximately realised in practice, but are sufficiently close to enable at least a rough forecast to be made of the intensity of coupling between two characters, if the intensity of their coupling with a third character is already known ${ }^{1}$.

1 In a case described by Sturtevant (Journ. Exp. Zool. xiv. p. 48) on 
The explanation of these facts offered by Prof. Morgan is of this kind. He assumes that when the chromosomes undergo side-to-side pairing in the zygotene stage, they donot simply lie parallel with each other, but become more or less twisted round each other like the strands in a rope, and that when the split takes place which separates the chromosomes in the first maturation (heterotype) division, the chromosomes do not unwind themselves, but the split is straight, so that part of what was one chromosome remains attached to the other, while a corresponding.part of the second becomes attached to the first. Such twisting of the zygotene chromosomes has been observed in several animals and plants, and while some observers maintain that they become untwisted as they shorten to form the heterotype "gemini," others (notably Janssens, who worked with Amphibia) describe a process exactly corresponding with what Morgan's hypothesis demands (cf. Text-fig. I3, p. 90). It is unfortunate, however, that hitherto nothing of the sort has been discovered in Drosopbila itself, perhaps in consequence of its unsuitability for cytological study.

Morgan thus assumes, firstly that when chromosome $A$ pairs longitudinally in synapsis with its homologue $a$, they become twisted round or across each other, and that instead of separating completely, a part of $A$ remains attached to $a$ and an exactly corresponding part of $a$ takes its place in $A$. Secondly, he assumes that the factors for the coupled characters are arranged in a definite order along the chromosomes like beads on a string; these factors in chromosome $A$ may be lettered $M, N, O \ldots$ and in its homologue $a$ the

which the above imaginary example is based, the average ratio of crossing-over between factors $A$ and $B$ was I per cent., between $B$ and $C 29^{\circ} 7$ per cent. and between $C$ and $D_{3}$ per cent. The percentage of crossing-over between $A$ and $C$ was found to be $32^{\circ} 2$, between $B$ and $D 33^{\circ} 7$, and between $A$ and $D 35.5$.

D. C. 
factors will be $m, n, 0 \ldots$. Then if the part of $A$ which contains the factor $M$ exchanges places with the part of $a$
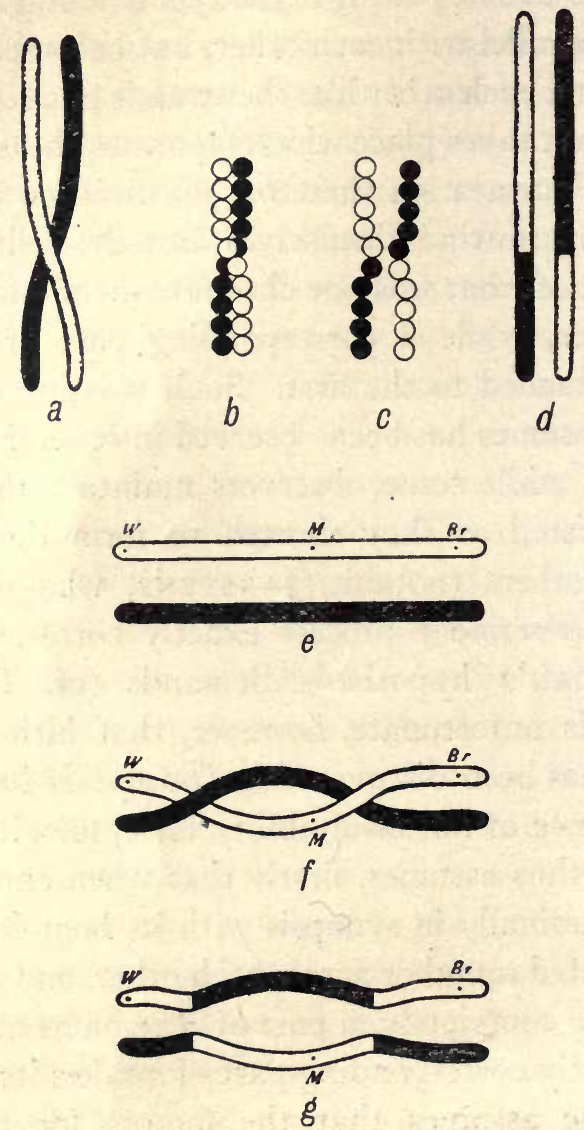

FIG. 28. Diagrams illustrating the hypothesis of "crossing-over" of characters borne by chromosomes, from Morgan.

$a-d$. Simple crossing-over; $b$ and $c$ represent the chromosome as made up of a series of microsomes each of which is supposed to bear one character.

$e-g$. Illustrating double crossing-over.

In consequence of the double cross-over, the character $M$ is transferred to the lower chromosome while $W$ and $B r$ remain in the upper. 
which contains $m$, the chromosomes on the spindles of the maturation divisions will contain factors $m, N, O \ldots$ and $M, n, o \ldots$; that is to say, $M$ and $m$ will have crossed over from their normal couplings, and will no longer be transmitted with the characters with which they were associated in the parents. Furthermore, if the factors $M, N, O, P \ldots$ are arranged along the chromosome in this order, and if the break in the chromosome occurs with equal frequency at any point, it will evidently occur more often between the widely separated factors $M$ and $P$ than between contiguous factors such as $M$ and $N$ or $O$ and $P$, and if it occurs in $x$ per cent. of cases between $M$ and $N$, and $y$ per cent. between $N$ and $O$, it will occur in $x+y$ per cent. between $M$ and $O$. That is to say, the frequency with which the break occurs between any two factors is proportional to their distance apart in the chromosome ${ }^{1}$. In all these respects the hypothesis corresponds closely with the facts. Again, if the chromosomes are sufficiently long, or if the twisting in synapsis is tight enough, it may be expected that more than one break may take place, which will result in an intermediate portion of chromosome $A$ being transferred to $a$ (and of $a$ to $A$ ), while the portions at each end of it remain in their original association (cf. Text-fig. 28). Such "double crossing-over" actually occurs in experiment; if an individual bearing the factors $M, N, O, P$ is mated with one bearing $m, n, o, p$, it is found that a small proportion of the gametes of the heterozygote have such combinations as $M, n, o, P$ and $m, N, O, p$, suggesting that there have been two breaks, one between $M$ and $N$ and one between $O$ and $P$, with the result that the intermediate portion of the chromosome, containing factors $N$ and $O$, has exchanged places

1 J. B. S. Haldane (Fourn. of Genetics, vili. 1919 , p. 299) shows that this is only approximately true. 
with that containing $n$ and $o$. And lastly, if a break occurs between $M$ and $N$, it is unlikely that another will take place between the contiguous factors $N$ and $O$, and correspondingly MORGAN and his associates give evidence of "interference" in crossing-over between two factors, in gametes in which crossing-over has taken place between two factors placed near them in the series.

One considerable objection to this hypothesis must be mentioned here, though further reference will be made to it later. Crossing-over in Drosopbila occurs only in the female; in the male characters that are normally coupled always show absolute coupling. In the Silk-worm, on the contrary, TANAKA has shown that there are several pairs of coupled characters, and that, while crossing-over takes place between them in the male, in the female the coupling is absolute. Although no cytological explanation of these facts is as yet definitely known, there are indications in Drosopbila of differences between the prophases of the male and female maturation stages which may account for them. A description of these will be given below (p. 235).

Apart from the fact that the theory of MoRGAN and his colleagues with regard to the mechanism of coupling or linkage and of crossing-over cannot be regarded as fully established until the difference in behaviour in this respect of the two sexes has been explained, the theory has also been criticised on other grounds. The most serious of these is that the facts are insufficient, as they are known at present, to provide an adequate basis for the hypothesis. There are undoubtedly a number of irregularities which involve secondary assumptions for their explanation; the ratios obtained in breeding experiments are often unconformable, requiring the assumption of differential mortality, of "lethal" factors, of factors modifying the intensity of coupling, and 
so forth. The evidence for some of these subsidiary hypotheses is strong, for others less satisfactory, but it remains true that no other hypothesis that has been suggested fits the main groups of facts in at all the same degree as that of Morgan and his school, and it must be concluded that the "chiasmatype" hypothesis, as Morgan prefers to call it, adopting the term from Janssens, is the only one which seems at all nearly to approach the truth.

Before leaving this section of the subject it should be noted that in Drosophila all the known Mendelian characters fall into one or other of the four groups of coupled characters, while in all other species hitherto investigated many or all of the characters show independent inheritance. This may be due to the small number (four) of the haploid chromosomes in Drosophila, but another possible explanation is that in Drosophila the chromosomes are perhaps only slightly twisted, so that only one or two breaks occur in each pair. If, however, they were more tightly twisted, so that a considerable but varying number of breaks and recombinations took place, it would be an even chance whether two factors not very near together in the chromosome remained together or became separated, and independent inheritance would result. In such a case coupling would only exist between factors that were arranged quite near one another in the chromosome.

The last and perhaps most important evidence in favour of the theory that the factors for inherited characters are borne by chromosomes is derived from the behaviour of the sex-chromosomes already described in Chapter XI. Here again, some of the most important work has been done in Prof. Morgan's laboratory, and although this aspect of the matter is not very intimately related to the chiasmatype hypothesis, the two are nevertheless closely connected. It 
will be remembered from the account of the cytological basis of sex-determination that in a number of insects, including the Diptera, as well as in other forms, the male has either an unpaired $X$-chromosome or an unequal $X-\gamma$ pair, while the female has two similar $X$-chromosomes, with the result that two kinds of spermatozoa are produced, one containing $X$ and onewithout it, while all eggs are $X$-bearing. From this it follows that individuals produced from eggs fertilised by an $X$-bearing spermatozoon contain two $X$ chromosomes in their nuclei, and become females, while those derived from eggs fertilised by a spermatozoon lacking $X$ contain only one $X$-chromosome, and are males. On the other hand, in two species of Lepidoptera it has been found that all the spermatozoa are alike, while there are two kinds of eggs, one containing and the other lacking a chromosome present in the spermatozoa, and in this case it seems that the presence of this chromosome in the egg before fertilisation leads to the production of a male. If, therefore, the hypothesis is correct that inherited, or at least Mendelian, characters are determined by factors borne by the chromosomes, it is to be expected that in those animals, such as Diptera and Mammals, in which some spermatozoa contain and others lack an $X$-chromosome, the factors for any characters borne by the $X$-chromosome will be transmitted by males only to their female offspring, while females will transmit them equally to their offspring of both sexes. And conversely in the Lepidoptera, in which the female-determining eggs are without one chromosome, the female should transmit any characters borne by. this chromosome only to their sons, while males should transmit them to both sexes. Inheritance of the kind described does exist, known as sex-limited or sex-linked transmission, and in Drosopbila among the Diptera, and in Man and the Cat 
among Mammals, it is of the kind in which the male transmits certain features only to his daughters, while in Lepidoptera and Birds the converse condition exists in which the female transmits characters only to her sons. In each case, therefore, the sex in which one chromosome is either unpaired, or has a dissimilar mate, transmits certain characters only to the opposite sex, exactly as the odd chromosome is transmitted to offspring of the other sex. In Drosopbila, for example, a red-eyed, normal-winged male mated to a white-eyed, rudimentary-winged female has red-eyed normal-winged daughters and white-eyed, rudimentary-winged sons. The transmission of these two characters thus corresponds with the fact that the male transmits the $X$-chromosome only to his daughters. In the converse cross of redeyed, normal female by white, rudimentary male, all the offspring of both sexes are red-eyed and normal-winged, since the female transmits an $X$-chromosome, which by hypothesis bears these characters, to her offspring of both sexes. Similarly in Man, the male transmits the factor for normal vision as contrasted with colour-blindness only to his daughters, and in the Cat the male transmits the orange colour in the same way, and it has been found that in several Mammals, including Man and the Cat ${ }^{1}$, an unpaired $X$ chromosome exists in the male and is therefore transmitted by him only to his female offspring.

In the Moth Abraxas, on the other hand, the conditions are reversed. A female of the type form mated with a lacticolor male has sons of the type form and lacticolor daughters, so that in this species the female transmits the type character only to her sons, while in the converse cross the male transmits the same character to both sons and daughters And correspondingly in some races of this moth

1 VON WiNIWARTER, 1912, 1914. 
there is an unpaired chromosome in the female, with the result that in the maturation of the eggs it is left in the eggnucleus of about half the eggs, and goes into the polar body in the other half. If this odd chromosome is a sex-determiner, its behaviour corresponds with the transmission of the type as contrasted with the lacticolor character. It should be mentioned, however, that there are peculiarities in regard to this chromosome which have hitherto received no satisfactory explanation ${ }^{\mathbf{1}}$.

This correspondence between the transmission of characters showing sex-limited (sex-linked) inheritance and of the sex-chromosome is so exact that it is hardly possible to doubt the existence of a causal relation between them, but the evidence has been still further strengthened by the work of Bridges in Morgan's laboratory. In most species in which sex-limited inheritance is known occasional exceptions occur, in which the male transmits a sex-linked character to a son, or (in Lepidoptera and Birds) in which a female transmits it to a daughter. Such exceptions have repeatedly occurred in experiments with Drosopbila, and BRIDGes first suggested that they might be due to "non-disjunction" of the sex-chromosomes, and then by cytological investigation showed that his hypothesis was correct. He suggested, for example, that if a red-eyed male, mated to a white-eyed female, produced a red-eyed son or a white-eyed daughter, this might be due to abnormal behaviour of the sex-chromosomes in the polar divisions of the egg, by which the two $X$-chromosomes after pairing failed to separate, and either both remain in the mature egg or are both extruded with the polar bodies. If both $X$-chromosomes (bearing the whiteeye factor) remain in the egg and the egg is fertilised by a $r$-bearing spermatozoon, the resulting zygote, of consti-

1 Doncaster, 1914, 19156. Cf. note, p. 171. 
tution $X X Y$, will be a female, since two $X$-chromosomes are present, but will be white-eyed because no red-bearing $X$-chromosome has been introduced from the male parent. And if both $X$-chromosomes are extruded, and the egg is fertilised by an $X$-bearing spermatozoon, the zygote, of
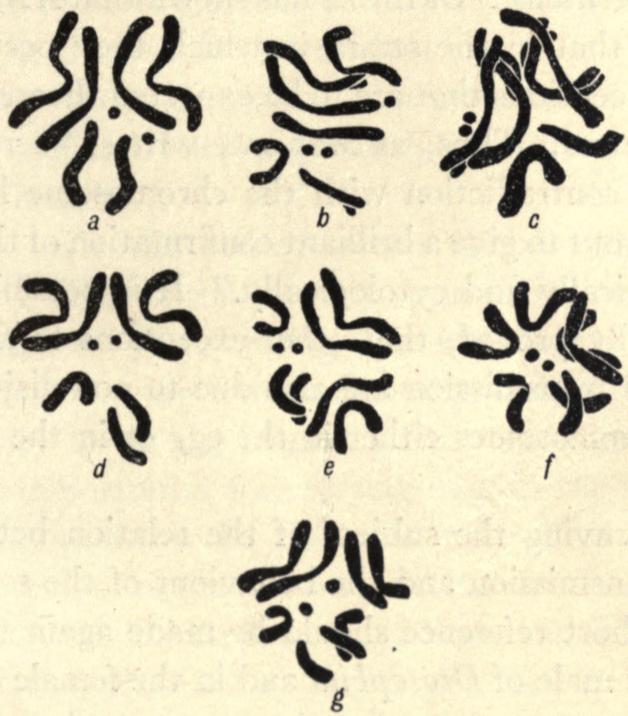

FIG. 29. Chromosome groups of Drosophila ampelophila (from original drawings by C. B. BRIDGES).

a. Normal oogonial group; the two lowest chromosomes are ' $X$.'

$b, c$. Normal spermatogonial groups. The bent chromosome (left bottom) is ' $Y$,' the straighter rod on the right of it, ' $X$.'

Some of the chromosomes are beginning to split longitudinally.

In $b$ "the extra granule is probably of no significance" (BRIDGES).

$d, e, f$. Oogonial groups of $X X Y$ females.

g. Oogonial group of an $X X Y r$ female.

constitution $X$-, will be male, but will be red-eyed because the single $X$-chromosome is derived from the red-eyed father. Such "primary non-disjunction" of the sex-chromosomes is very rare, but when it has once happened it leads to the production of females of the constitution $X X Y$, and 
these will continue to produce sons and daughters that are exceptions to the normal rule of sex-limited transmission. For a female of constitution $X X Y$ will produce four kinds of mature eggs, bearing $X Y, X, X X$, and $Y$, and of these the $X X$ and $r$ eggs will produce exceptions to the rule of sexlimited inheritance. BrIDges has shown that $X X Y$ females exist, and that in the strain in which they occur all the kinds of exceptions that are to be expected theoretically are actually found. Thus, as Morgan writes, "a result that seemed in contradiction with the chromosome hypothesis has.turned out to give a brilliant confirmation of that theory both genetically and cytologically." It is possible, though not yet fully proved, that other exceptions to the rule of sex-limited transmission are also due to non-disjunction of the sex-chromosomes either in the egg or in the spermatocytes.

Before leaving the subject of the relation between sexlimited transmission and the behaviour of the sex-chromosomes, a short reference should be made again to the fact that in the male of Drosophila and in the female Silk-worm no crossing-over takes place between coupled characters, although it occurs regularly between these characters in the opposite sexes. If this were true only of characters that show sex-limited transmission, the fact would be easily explicable on the assumption that no crossing-over could take place between unequally paired sex-chromosomes such as exist in the male of Drosopbila and probably (by inference) in the female Silk-worm. But the absence of crossing-over in the sex that shows sex-limited transmission is equally conspicuous in characters that are not in any way sexlimited, and it must be concluded that the existence of an unpaired or unequaily paired sex-chromosome in some way inhibits the crossing-over of coupled characters in all the 
chromosomes. The more fundamental reasons for this difference between the sexes is still quite obscure, but indications of the immediate cause of it are given by the observations of C. W. Metz on Drosophila.

He writes " "In the female the critical stages of maturation appear to be of the conventional type. That is, long, delicate paired threads are evident early in the growth period. These are polarised [i.e. contracted to one side of the nucleus in synizesis] and apparently conform to the usual diplotene threads....

"In the male the process seems to be very different throughout. The chromosomes apparently remain in a fairly condensed state during the whole growth period, without transforming into delicate polarised threads or loops as they do in the female.... This would indicate that there is no opportunity for crossing over in the male."

In confirmation of the fact that this difference in the nuclear behaviour of the two sexes in Drosophila is the cause of the difference in respect of crossing-over, it should be mentioned that Plough (1917) finds that by subjecting female pupae to different temperatures the amount of crossing-over can be modified, and his experiments indicate that crossing-over must take place in the early stages of the oocytes-those stages, in fact, in which METz reports differences in nuclear behaviour in the two sexes.

Finally, a short space must be devoted to the hypotheses or suggestions that have been made with regard to mode of action of the chromosomes in determining sex and in transmitting the factors for Mendelian characters. It has been seen that in most Insects and in animals of a number of other groups the male has one $X$-chromosome, either

1 Quotation from a letter which the writer kindly gives permission to publish. 
unpaired or accompanied by a dissimilar mate $(\eta)$, while the female has two similar $X$-chromosomes. On the other hand, in Lepidoptera (certainly in two cases) and in Birds (by inference from the nature of their sex-limited transmission) the male has two similar sex-chromosomes and the female one or a dissimilar pair ${ }^{1}$. Two chief hypotheses have been suggested to explain their manner of action. One was that in the former group the two $X$-chromosomes of the female bore respectively the factors for maleness and femaleness, femaleness being dominant, and the single $X$-chromosome of the male bore only maleness. There would thus be two kinds of eggs, which may be called $M$ and $F$, and two kinds of spermatozoa, $M$ and $O(O$ representing the absence of an $X$-chromosome). If then there is selective fertilisation of such a kind that $F$-bearing eggs are only fertilised by $M$ bearing spermatozoa, and $M$-bearing eggs only by $O$ bearing spermatozoa, two kinds of zygotes, $F M$ and $M O$, will be produced, and these will become females and males of the same constitution as their parents. In the Lepidoptera and Birds maleness instead of femaleness must be supposed to be dominant, and the male would have the constitution $M F$, the female $F O ; F$-bearing eggs would then be fertilised by $M$-bearing spermatozoa, and $O$-bearing eggs by $F$-bearing spermatozoa. The great objection to this hypothesis is that it involves the assumption of selective fertilisation, for which there is practically no evidence, and it is now generally abandoned in favour of a somewhat simpler conception-that there are not two factors, for maleness and femaleness, but that the sex depends on the presence of a

1 GuYer maintains that in the Fowl there is an unpaired chromosome in the male. In view of the great difficulty in observing the chromosomes accurately in the spermatogenesis of birds, I venture to doubt the correctness of his statement, which has not been confirmed. 
greater or less amount of a sex-determining substance borne by the sex-chromosomes. In the groups in which the female has the constitution $X X$ and the male $X$-or $X . r$, femaleness is supposed to be due to the "double dose" of $X$-chromatin, while in the Lepidoptera and Birds males are produced by a double dose of a (presumably) different substance in the chromosome that is equally paired in the male and not in the female. This idea that sex is due to a quantitative rather than a qualitative difference in the chromosome complement is not only simpler than the other, but is supported by those cases (for example, several moths) in which the crossing of two species produces either all males, or males and gynandromorphs, when the cross is made one way, but both males and females in the converse cross. This suggests that the spermatozoa of one species introduce so much of the sexdetermining substance that all the eggs, whether they contain a sex-chromosome or not, are partially or completely turned into males, while in the converse cross the less potent spermatozoa of the second species allow the sex to be determined by the presence or absence of a sex-chromosome in the egg.

The most important evidence in this direction has been furnished byGoLDSCHMIDT's(I9I6) remarkable experiments with races of the Moth Lymantria dispar. He concludes that a male-determining factor $M$, of varying potency, is borne by a sex-chromosome, and is opposed to a female-determining factor $F$ borne by the egg-cytoplasm. When there are two sex-chromosomes, as there are in the male of Lepidoptera, $M M$ overpowers $F$, and the individual is a male, while when there is only one $M$ chromosome, as in the female, $F$ overpowers $M$. Both $F$ and $M$ vary in potency in different races, so that by crossing selected races it is possible to get individuals in which the potency of $F$ equals that of 
$M$, with the result that "female intersexes" (combining the characters of both sexes) are produced. He further finds that the potency of $M$ behaves as a Mendelian character, but that the potency of $F$ is transmitted only by the female, and remains constant in all descendants in the female line. From this he infers that $F$ is a cytoplasmic factor, borne by the egg and not by the spermatozoon.

A very nearly related question is the precise meaning to be attached to the statement that "the factors for sex or for Mendelian characters are borne by chromosomes." Evidence will be given in the following chapter that the cytoplasm is not without influence in the development of inherited characters, and this fact has been held to disprove the chromosome hypothesis. But a chromosome can obviously act only in its relation with the cytoplasm, and if the cytoplasm is altered this relation must evidently change with it. When, therefore, it is said that chromosomes bear the factors for characters it is implied that these factors are in relation with the cytoplasm, and will only produce their effects if that relation exists. A simple chemical analogy may make the conception clearer. If in the hydrocarbon $\mathrm{CH}_{3} \mathrm{H}$ the fourth hydrogen atom is replaced by chlorine $\left(\mathrm{CH}_{3} \mathrm{Cl}\right)$ or by hydroxyl $\left(\mathrm{CH}_{3} \mathrm{OH}\right)$, substances of very different properties are produced. A quite parallel series is represented by the compounds $\mathrm{C}_{2} \mathrm{H}_{5} \mathrm{H}, \mathrm{C}_{2} \mathrm{H}_{5} \mathrm{Cl}$, $\mathrm{C}_{2} \mathrm{H}_{5} \mathrm{OH}$, and although the two series of substances are not identical, it is perfectly correct to say that the change from a hydrocarbon to a chloride or an alcohol is due to the substitution of a hydrogen atom by one of chlorine or by the hydroxyl radicle. The cytoplasm, in this analogy, is represented by the hydrocarbon radicles, $\mathrm{CH}_{3}-\mathrm{C}_{2} \mathrm{H}_{5}-$ and so forth, and the effects on the general nature of the resulting compound of substituting $-\mathrm{Cl}$ or $-\mathrm{OH}$ for the $-\mathrm{H}$ 
of the hydrocarbon represents the effect produced by substituting a chromosome "bearing" one factor for a chromosome bearing another. Just as the hydrocarbon radicle is not negligible, since the compounds $\mathrm{CH}_{3} \mathrm{Cl}$ and $\mathrm{CH}_{3} \mathrm{OH}$ differ considerably from $\mathrm{C}_{2} \mathrm{H}_{5} \mathrm{Cl}$ and $\mathrm{C}_{2} \mathrm{H}_{5} \mathrm{OH}$, so the cytoplasm is not in any sense a negligible factor, but it is nevertheless true to say that the substitution of a hydrogen atom by a hydroxyl radicle $(-\mathrm{H}$ by $-\mathrm{OH})$ turns a hydrocarbon into an alcohol, and in the same way it may be true to say that the replacement of one chromosome by another in cytoplasm of a generally similar character may be the cause of the production of a definite inherited character. If the words are used in some such sense as this, the statement that inherited characters are due to factors borne by chromosomes, whether true or false on other grounds, cannot be refuted by the argument that the cytoplasm is not without effect in hereditary transmission.

Quite shortly, the evidence for the transmission of hereditary characters by chromosomes may be summarised as follows: (I) the nucleus must be the chief factor in hereditary transmission, since the spermatozoon consists of little else, and yet in respect of most characters the male and female parents are equivalent. Also experiments with non-nucleated fragments of eggs, and with "partial fertilisation," indicates that the nucleus plays a predominant part in the production of certain characters.

(2) A definite relation between abnormal chromosome numbers and hereditary characters is known in Oenothera; and in the giant tetraploid race of Primula studied by GREGORY, the peculiar type of Mendelian segregation corresponds with the behaviour of the chromosomes.

(3) The whole behaviour of the chromosomes in the maturation divisions of the germ-cells provides exactly the 
mechanism required for the Mendelian segregation of characters, and when, as in FEDERLEY's moth-hybrids, the normal segregation of chromosomes did not take place, there was also no Mendelian segregation. This correspondence of chromosome behaviour with transmission of hereditary characters is even more striking in the case of the sex-chromosome and characters which show sex-limited (sex-linked) transmission, and perhaps the most remarkable correspondence of all is that seen in the exceptions to normal sex-limited transmission, found by BRIDGEs to be associated with "non-disjunction" of the sex-chromosomes.

(4) Finally, the phenomena of gametic coupling or linkage of characters have been found, in the work of Morgan and others on Drosopbila, to correspond closely with the chromosome-condition, for in Drosopbila there are four groups of linked characters and four pairs of chromosomes, and all the phenomena of "crossing-over" can be accounted for on a simple hypothesis of chromosome behaviour. 


\section{CHAPTER XV}

\section{The Rôle of the Cytoplasm in Development and Heredity}

7 o some it may seem that the evidence for the preponderating part played by the chromosomes in the transmission of inherited characters is so strong that the cytoplasm must of necessity be regarded as taking a subordinate place, and as constituting the medium in which the chromosomes display their activities rather than having any independent function. A careful survey of the evidence, however, shows that this conclusion is by no means justified. In the first place, the arguments pointing to the primary importance of the chromosomes refer almost entirely to the transmission of characters which show Mendelian segregation, and the suggestion has therefore been made that the factors for Mendelian characters are situated in chromosomes, while those which do not show Mendelian segregation are borne by the cytoplasm. It is said (for example, by WALKER) that Mendelian characters are varietal, and that racial or specific characters, which are supposed by some not to "mendelize," are borne by the cell as a whole rather than by particular chromosomes. For such a statement as this there is very little real evidence; it is, in fact, by no means certain that there is any heredity other than Mendelian, for when characters apparently alternative to each other do not segregate in the typical way, this may be due to their being. in reality compound; or in crosses between more distantly related forms the absence of segregation may be due to the

D. c. 
incompatibility of the parental chromosomes, as in FEDERLEY's Pygaeras. The hypothesis must therefore remain little more than a speculation until both the genetic and cytological facts are better known. Goldschmidt's idea of a female determining factor borne by the cytoplasm in Lepidoptera might be quoted in support of this hypothesis.

Another argument that has been adduced in favour of the importance of the cytoplasm, or at least against the exclusive rôle of the chromosomes, in heredity, is that crosses between distantly related forms may give offspring of the pure maternal type. A well-known instance of this is provided by GoDLEwskr's experiment of fertilising the eggs of Ecbinus with sperm of the Crinoid Antedon, by which he got pluteus larvae showing no trace of Crinoid characters. He also fertilised non-nucleated fragments of Echinus eggs with Antedon sperm, and obtained blastulae and a few gastrulae of the Echinoid type, though these never developed any skeletal structures. Since the Antedon chromosomes were not extruded, but apparently behaved normally in the segmentation divisions, this experiment has often been used as an argument that the chromosomes do not determine inherited characters, or at least those characters which distinguish one Order from another. Similar results have been observed (for example by LоEв) in crosses between Seaurchins and Starfish and by PIN NEY in Teleostean hybrids. As was mentioned in Chapter IX, however, it is known that the entrance of a spermatozoon may stimulate an egg to develop even though the sperm-nucleus takes no part in the cell-divisions, as happens when Sea-urchin eggs are fertilised with Mytilus sperm, and there seems to be no sufficient reason against regarding these purely maternal "hybrids" as something of the same sort. It may be supposed, for example, that when forms are crossed in which there is a 
high degree of incompatibility between the egg-cytoplasm and the sperm-nucleus (e.g. Echinoid eggs and Mollusc sperm), the sperm-nucleus is unable to resolve itself into chromosomes, or, if it does so, that these take no part in mitosis. If the incompatibility be rather less, the chromosomes may be formed to all appearance normally, and divide in the usual way on the spindle, but nevertheless they may be so alien to the cytoplasm in which they exist, that they exert no influence upon it, and the characters of the resulting larva are as they would be if the egg developed parthenogenetically, that is to say, purely maternal.

Finally, if the incompatibility is relatively slight, the chromosomes may be able to adapt themselves to their strange surroundings, and not only continue to live and divide, but also to have some effect on the characters of the larvae or adults produced, as happens in generic crosses between Sea-urchins. It is impossible at present either to prove or to disprove this suggestion, but until it is disproved such results as those obtained by GoDLEwski cannotbeused as a final argument against the chromosome hypothesis, at least with regard to characters that appear later than the very early larval stages. The observation that gastrulae from non-nucleated Echinoid egg-fragments fertilised by Antedon sperm are of the Echinoid rather than of the Crinoid form does of course disprove that all characters, at every stage of development, are determined by factors borne by chromosomes, but as will be seen immediately, there is ample experimental evidence to show that the very early stages of development are controlled largely, if not completely, by the egg cytoplasm.

Although, therefore, several of the experiments that are often quoted as showing that the cytoplasm is no less important than the chromosomes in transmitting inherited

$$
\text { r6-2 }
$$


characters do not necessarily prove this conclusion, there are a number of observations which indicate that the cytoplasm is not a negligible factor.

In the first place it is clear that the quite early stages of development, including cleavage and the appearance of embryonic symmetry, are in most animals, perhaps in all, determined by the egg, and from their correspondence with the egg-structure, almost certainly by its cytoplasm. All eggs show a definite polarity, corresponding with the anterior and posterior ends of the future larvae, and in several groups of animals, such as Insects, the egg also has a bilateral structure, which, quite apart from the spermatozoon, determines the orientation of the larva to which it gives rise. Not infrequently, also, the egg contains areas or zones of different substances and a study of the development shows that these become distributed quite regularly to definite organs or tissues, and although the experimental rearrangement of these visible substances (as by centrifuging) may show that the orientation of parts in the larva depends on the polarity of the egg as a whole rather than on the specific substances, it seems indubitable that the process of segmentation and differentiation of the parts of the embryo in many species is conditioned by the egg-cytoplasm and influenced scarcely, if at all, by the spermatozoon. It is of course not easy to devise an experiment that shall prove this directly, for the type of cleavage is in general characteristic of large groups, and so it is hardly possible to cross species of different types in order to see whether the cleavage of the hybrid is different from that of the female parent's species.

About the rather later stages of development there has been some difference of opinion. It is said by several investigators, for example Driesch, that in Echinoids the number and mode of formation of the primary mesenchyme cells 
is a purely maternal character, and apparently dependent on the egg-cytoplasm, but Boveri maintains that on this feature the spermatozoon has at least some effect, the number being greater in a hybrid in which the paternal species has more than the maternal.

Another kind of cytoplasmic influence may arise from the direct transference to the offspring of structures or subtances present in the egg-cytoplasm. The eggs of the Seaurchin Arbacia contain much red pigment, and in consequence all hybrid larvae produced from Arbacia eggs are pigmented, while those produced from the converse cross may be colourless. And in plants the chloroplasts are transmitted only in this way; if the cytoplasm of the egg-cell contains chloroplasts these are present and multiply in the cells of the embryo, but an egg-cell lacking chloroplasts gives rise to offspring that are totally without chlorophyll.

Apart from these rather special instances of characters dependent exclusively on the cytoplasm or its inclusions, there is evidence of a more general character that the cytoplasm affects development independently of the chromosomes, although it may be that in consequence of the interaction between chromosomes and cytoplasm, some of these effects may have their origin in the chromatin-cytoplasm relation rather than in the cytoplasm itself. In Weismann's hypothesis of the germ-plasm it was supposed that all differentiation of cells or tissues arose from differential celldivisions in which the chromosomes of the two daughter nuclei were in some way unlike. But except in the few cases of germ-cell differentiation referred to in Chapter XII, there is little or no evidence for the existence of such differential nuclear divisions. One of the chief characteristics of the chromosomes is that they appear to divide in mitosis into longitudinal halves which are precisely similar to each other, 
and this fact alone would suggest that the cytoplasm must have an important share in the differentiation of cells and tissues from the relatively unmodified protoplasm of the egg. There is, however, definite evidence that this is true.
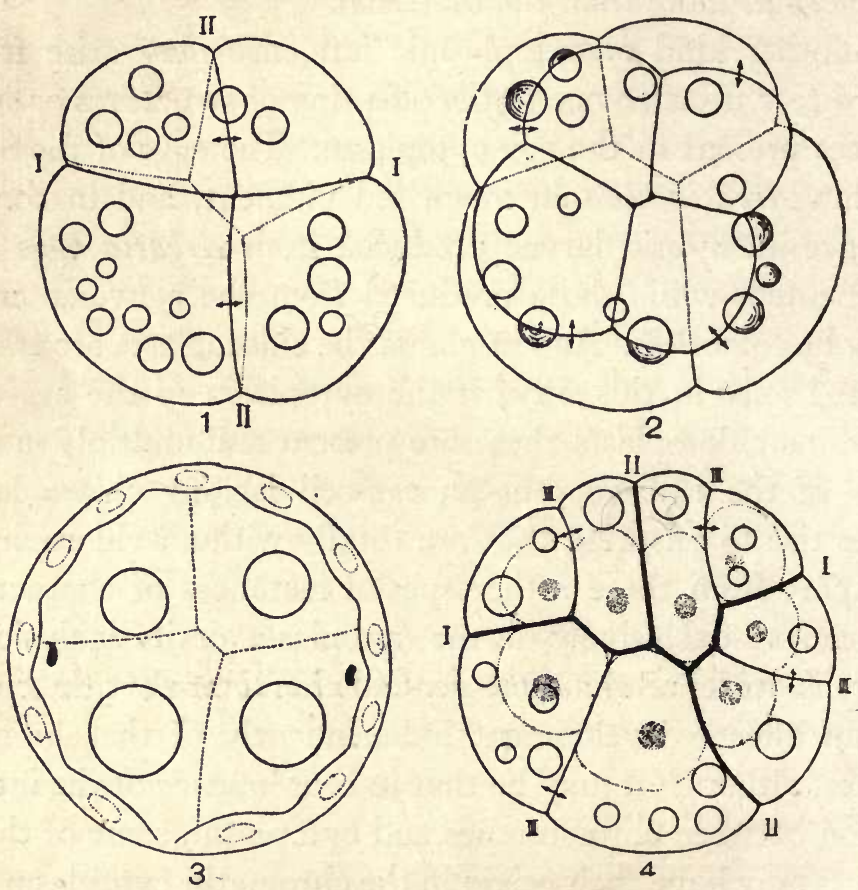

Fig. 30. Segmenting eggs of Nereis, from Wilson (1 896 ).

1. Normal four-cell stage. 2. Normal eight-cell stage; first quartet of micromeres above. 3. Embryo with four entomeres each containing a large oil drop, and twelve outer cells. 4. Eight-cell stage produced under pressure. The dotted lines show the outlines of the micromeres formed after release from pressure.

WILson (1896) in a well-known experiment on the eggs of the worm Nereis showed that nuclei which under natural circumstances would have been those of ectoderm cells could be made to belong to endoderm cells. If allowed to develop normally, the egg of Nereis segments by two cleavages into 
four cells, each of which then undergoes a very unequal division, giving rise to a small cell almost free from yolk and a large yolk-laden cell. Two similar unequal divisions of the large cells then follow, producing in all three quartets of small cells and four large cells containing yolk (oil-drops). The cap of small cells gives rise to the ectoderm; the four large cells undergo no further division for some time, and are the primary endoderm cells. If, however, the egg before segmentation is subjected to pressure between glass plates, all the cleavage furrows are vertical and the cells produced lie in one plane, so that after three cleavages there are eight cells all containing oil-drops. If they are now released from pressure, each cell divides unequally into an upper and Lower portion, giving rise to eight small cells and eight large oil-laden cells. The small cells then give origin to the ectoderm, and the eight large cells all become endodermal. It is evident, therefore, that the nuclear division of the third cleavage, which under natural circumstances would have separated ectodermal from endodermal cells, under these circumstances has not produced any differentiation, for all the eight cells become endoderm. In other words, the nuclei of four of the eight endoderm cells were naturally destined to become those of ectoderm, but actually, under experimental conditions, become part of the endoderm, from which it follows that the differentiation of the germ-layers is not conditioned by differential nuclear division, but by unequal division of the cytoplasm.

Many experiments have been made in order to determine the function of the various substances present in different parts of the egg of many animals, and from the regularity with which these are often distributed to definite organs or tissues, it has been concluded that they are specific "organ-forming" substances. Of the Ascidian Styela, for 
example, Conklin writes as follows: "There are four or five substances in the egg which differ in color, so that their distribution to different regions of the egg and to different cleavage cells may be easily followed, and even photographed, while in the living condition. The peripheral layer of protoplasm is yellow and it gathers at the lower pole of the egg, where the sperm enters, forming a yellow cap. This yellow substance then moves, following the sperm nucleus, up to the equator of the egg on the posterior side and there forms a yellow crescent extending around the posterior side of the egg just below the equator. On the anterior side of the egg a gray crescent is formed in a somewhat similar manner and at the lower pole between these two crescents is a slate-blue substance, while at the upper pole is an area of colorless protoplasm. The yellow crescent goes in to cleavage cells which become muscle and mesoderm, the gray crescent into cells which become nervous system and notochord, the slate-blue substance into endoderm cells and the colorless substance into ectoderm cells.

"Thus within a few minutes after the fertilization of the egg, and before or immediately after the first cleavage, the anterior and posterior, dorsal and ventral, right and left poles are clearly distinguishable, and the substances which will give rise to ectoderm, endoderm, mesoderm, muscles, notochord and nervous system are plainly visible in their characteristic positions.

"At the first cleavage of the egg each of these substances is divided into right and left halves. The second cleavage cuts off two anterior cells containing the gray crescent from two posterior ones containing the yellow crescent. The third cleavage separates the colorless protoplasm in the upper hemisphere from the slate-blue in the lower. And at every successive cleavage the cytoplasmic substances are segre- 

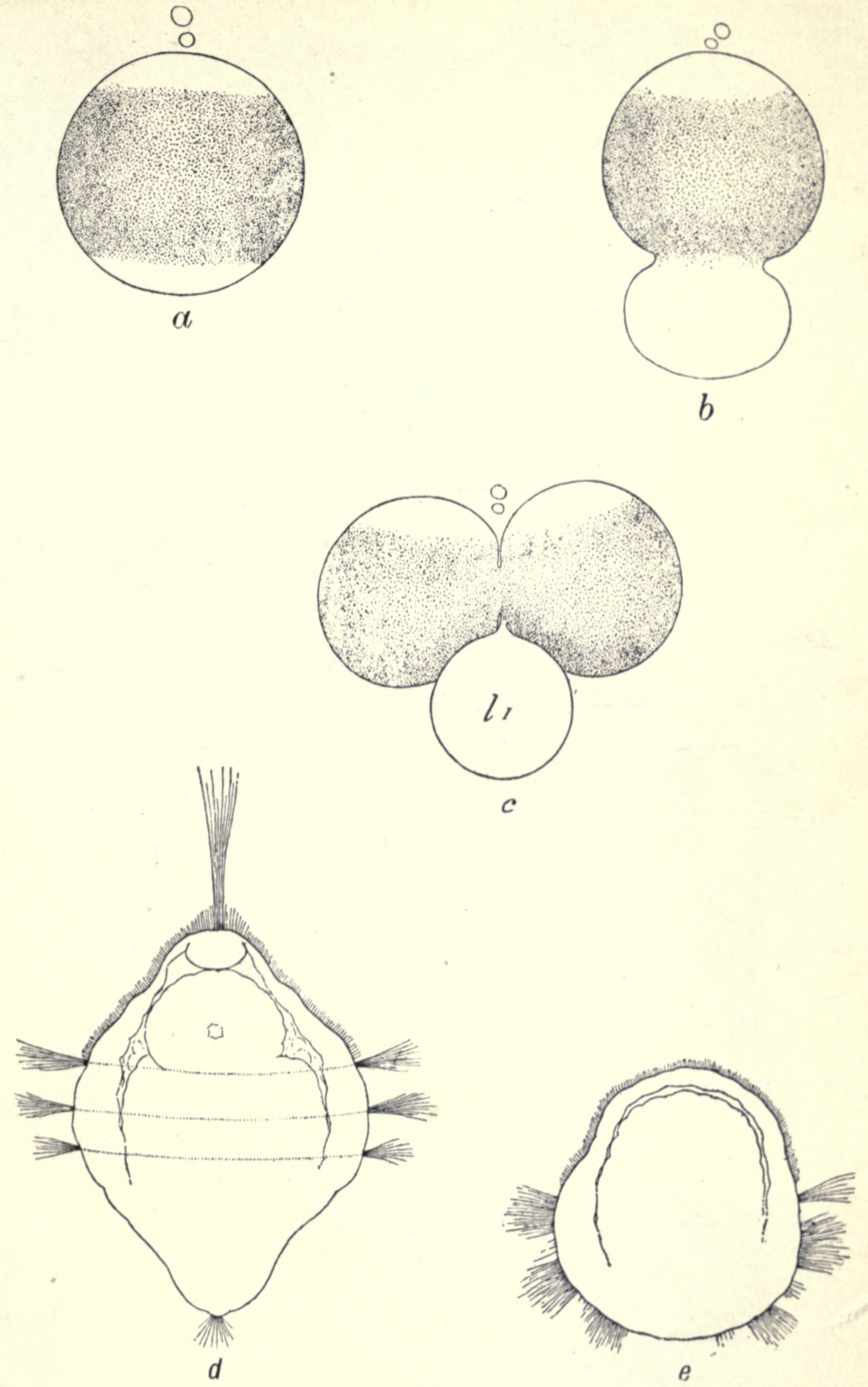

Development of Dentalium, from WiLson (1904).

a. Egg before development with upper and lower clear zones.

b. Formation of the yolk-lobe at the lower pole.

c. First segmentation division; the yolk-lobe is not divided.

d. Normal larva with anterior tuft of cilia and post-trochal cone.

e. Larva derived from an egg from which the yolk-lobe was cut off. 

gated and isolated in particular cells, and in this way the cytoplasm of the different cells comes to be unlike. When once partition walls have been formed between cells the substances in the different cells are permanently separated so that they can no longer commingle'."

Styela only offers a conspicuous example of a phenomenon that is widespread, for organ-forming substances have been described in the eggs and embryos of animals of very different groups. Sometimes there appears to be no immediate connection between the substance and the organ or region whose presence it determines. The egg of the Mollusc Dentalium, for example, has a broad equatorial band of reddish pigment, leaving small white anterior and posterior polar areas. In the first cleavage the posterior white area is not divided, but becomes a "yolk-lobe" attached to the two first cleavage cells (P1. XXIV). If before segmentation this white area is cut off, no yolk-lobe is formed, and in the resulting larva not only the post-trochal region, but also the apical tuft of cilia, is absent, so that it would appear that the substance of the yolk-lobe determines the production of parts at opposite ends of the larva (WILson, 1904).

Such observations as these, if taken by themselves, would point to the conclusion that the visible substances of the egg are in fact "organ-forming," that is to say, that they are or contain the determinants for the organs with which they are associated. But the experiments of Morgan (I909, I9I0), Boveri (I9IO), and others in centrifuging eggs that have visible differentiation into regions show that this conclusion is not justifiable in its simple form. Morgan has centrifuged the eggs of the Frog, the Sea-urchin Arbacia, the Mollusc Cumingia, the Rotifer Hydatina, and other forms, and obtains results in general agreement, indicating that

1 Conklin, 1918, pp. $123-125$. 
"the visible substances of the egg that can be centrifuged are not organ-forming" (1910, p. 613). In Arbacia the normal axis of the egg is marked by a funnel in the outer membrane, and it was found that the stratification produced by centrifuging had no constant relation to the original axis.

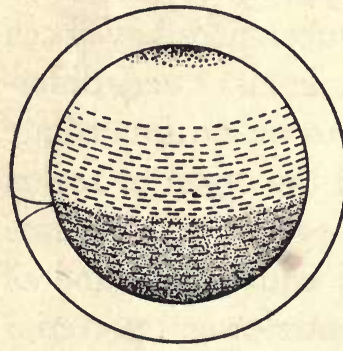

I

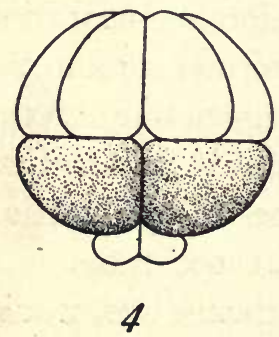

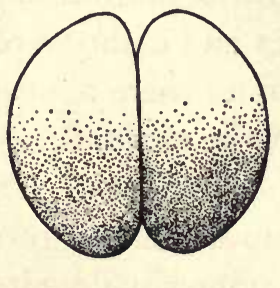

2

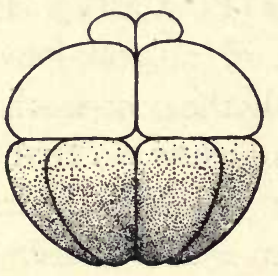

5

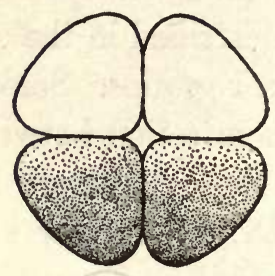

3

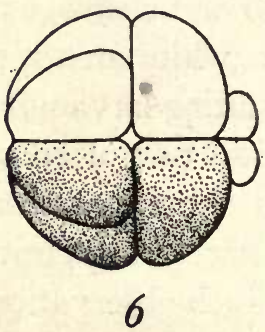

FIG. 31. Segmentation of centrifuged eggs of the Sea-urchin Arbacia, from MORGAN (1 909).

1. Centrifuged egg before segmentation; the attachment funnel on the left marks the original axis of the egg. 2, 3. First and second cleavages, determined by induced polarity. 4, 5, 6. Fourth cleavage; the position of the micromeres depends on the original polarity, and not on that induced by centrifuging.

"The first cleavage begins, almost invariably, at the white cap where the nucleus lies and cuts through the egg, dividing the stratified materials at right angles. The second plane is at right angles to the first, lying near the line of separation of yolk and clear zone, and the third at right 
angles to both. The fourth cleavage is a differential cleavage. Four micromeres appear at one pole; the cells of the opposite hemisphere divide equally. This cleavage is a critical one, for the micromeres show in the normal egg where the digestive tract will develop. The micromeres become the mesenchyme."

In the centrifuged eggs it was determined that the archenteron develops at the micromere pole, and that the micromeres lie opposite to the funnel in the envelope, independently of the secondarily induced axis. "In other words, while the first three cleavages come in with respect to the stratification, the fourth or differential cleavage comes in with respect to the original egg-axis." Normal plutei were obtained from such eggs, in some of which the red pigment was contained in one region, in others, in other regions.

Morgan's experiments on Cumingia and other forms, and Boveri's on Ascaris also lead to the same conclusion, that in general the differentiation of parts in the embryo is not dependent on the visible substances of the egg, but on an invisible polarity that is independent of the substances which can be moved or transposed by the centrifuge.

Conklin (1917) explains this polarity as being due to the highly viscid or elastic "spongioplasm" of the egg, "which always tends to comeback to its normal form if it is stretched or distorted, and which probably differs in structure in different parts of the egg and in different stages of development." And that the visible substances which distinguish the regions of the egg, especially in Tunicates (e.g. in Styela mentioned above), are not in themselves "organ-forming" is further indicated by the work of DUESBERG and of HirschLER, quoted by GATENBY (1919b) and of GATENBY himself, for these investigators find that the various regions of the

1 Morgan, I909, p. 157. 
egg owe their distinctive colours to the presence of mitochondria, Golgi elements and a certain amount of yolk in the different regions. It seems much more probable that these formative substances are present in regions which in normal development will give rise to parts where they are needed, than that their presence in itself in those parts should be the cause of specific differentiation.

Further support for this idea of polarity independent of visible substances may be derived from Driesch's famous experiments on Sea-urchin embryos. He finds that if, before any great differentiation has taken place, the embryo is divided in any plane, the fragments, if large enough, will produce complete larvae. From this it follows that a cell which in natural conditions would have formed part of one organ, in the divided embryo becomes part of another. The fate of a cell thus depends not only on its own inherent properties, but also on its position with respect to other surrounding cells, and this seems to involve the conclusion that the larva as a whole has some sort of polarity which to some extent at least is independent of the character of the separate cells of which it is composed, and therefore independent of any factors which may be borne specifically by their nuclei.

From facts of the kind just mentioned,Driesch has drawn the conclusion that living organisms differ fundamentally from all non-living matter in their activities, for while all changes in non-living matter are mechanical, that is, dependent solely on chemical and physical forces, he considers that it is impossible for any mechanism to bring about the results observed in living beings. A Sea-urchin embryo can be divided in its early stages in any direction, and the parts, if large enough, will produce complete larvae, and DRIESCH maintains that no conceivable mechanism can be so divided 
and remain complete after division. It seems evident that only by some inherent polarity existing in all the parts of the embryo can such a result be achieved, but whether such polarity is or is not conceivable on a purely physico-chemical basis is a question too large to be discussed here. It should be noted, however, that experiments on Echinoid embryos and other forms showing analogous phenomena provide only one of several arguments brought forward by DRIESCH in support of his belief in an extra-physical (nonmaterial) factor in the development and behaviour of living beings, although if this particular argument should be shown to be non-valid, the others would be greatly weakened if not completely destroyed.

Although in Echinoderm embryos, and in those of several other forms, the parts are equipotential during the first cleavages - that is, each can, under experimental conditions, give rise to any part of the larva - this is not true of the eggs or early embryos of many other forms. In such Molluscs as Dentalium the removal of part of the egg before segmentation causes the larva to be defective, and in Ctenophores, if the first two blastomeres are separated, each produces only a half-larva. Facts of this kind have been used to show that Driesch is mistaken, but, as he shows quite clearly, this objection is not valid. In any embryo, whether the parts in the early stages are equipotential or not, a stage comes sooner or later in which differentiation has gone so far that any cell or group of cells is only able to produce certain parts and not others; in Echinoderms, for example, when the cells that will naturally form endoderm are sufficiently differentiated, their removal results in the production of a larva lacking the archenteron. And such eggs as those of Molluscs or Ctenophores, in which each blastomere will only produce those parts which naturally arise from it, 
differ from Echinoid eggs, not in the fact that in one the parts are definitely determined while in the other they are more or less indeterminate, but that in the one determination appears early and in the other later. At some stage in every egg, so far as is known, the parts are equipotential, and from such a homogeneous egg heterogeneity arises sooner or later. Fundamentally, it is on this production of heterogeneity from the homogeneous apart from external influence that DrIEsch bases his belief in the inadequacy of physico-chemical forces to explain development.

The general conclusion to be reached with regard to the function of the cytoplasm in development and inheritance is that it cannot be relegated to the position of a mere medium in which the chromosomes carry on their activities, but that it plays an essential part in the process. By the nature of the case it is not possible to ascribe to it definite functions in the same way that it may be assumed, with something like probability, that the chromosomes are responsible for the segregation of Mendelian characters in inheritance. The chromosomes are definite entities, possibly - even probably-retaining their individuality from one cell generation to the next, while the cytoplasm is a complex in which, at present, it has not been possible as a rule to observe the different parts separately and continuously. But the chromosomes and cytoplasm together are parts of a continuous whole; it is no more possible to regard the chromosomes as acting independently than to think of the brain functioning apart from the body, and perhaps the crude chemical analogy of the parallel series $\mathrm{CH}_{3} \mathrm{H}, \mathrm{CH}_{3} \mathrm{Cl}$, $\mathrm{CH}_{3} \mathrm{OH}$ and $\mathrm{C}_{2} \mathrm{H}_{5} \mathrm{H}, \mathrm{C}_{2} \mathrm{H}_{5} \mathrm{Cl}, \mathrm{C}_{2} \mathrm{H}_{5} \mathrm{OH}$, given in the preceding chapter, expresses the idea of the relation as well as any single metaphor that can be devised. But this analogy fails in one important respect, for it can hardly be doubted 
that in the interaction that takes place between nucleus and cytoplasm, each side is more or less modified by the other. If chromosomes of Strongylocentrotus are introduced into Sphaerechinus cytoplasm by fertilising Sphaerechinus eggs by Strongylocentrotus sperm, it can hardly be doubted that the cytoplasm of the larva is modified by the paternal chromosomes, and it is possible, though less easily demonstrable, that the chromosomes of paternal origin also undergo changes, and that if such a hybrid could be crossed back with pure Strongylocentrotus, no larvae with quite unmodified Strongylocentrotus characters would be produced. Such an experiment is probably impossible to perform, for in cases in which the forms crossed differ in only one or few characters, their general constitution is so alike that no modification of the chromosomes by the cytoplasm is likely to appear. When, however, the forms crossed are sufficiently unlike to make such modification probable, even if the hybrids were fertile the segregating characters would be so numerous that complete analysis of them would be impossible. But if it be true that the factors for Mendelian characters are borne by chromosomes, these factors can only cause the characters to appear by producing modifications in the cytoplasm, and if the chromosomes modify the cytoplasm, it seems natural to assume that the relation is a reciprocal one, and that the cytoplasm may be not without effect on the chromosomes. That the effect may be relatively small is indicated by the persistence of chromosomes of the paternal type through the whole life-history of certain hybrids (for example the moths Biston hirtarius and Nyssia zonaria referred to in Chapter VI), but because the modification is not large or conspicuous it is not necessarily nonexistent. A wide field for research remains open in the investigation of the means and results of this interaction between the nuclear and cytoplasmic constituents of the cell. 


\section{CHAPTER XVI}

\section{Conclusion}

TN most of the preceding chapters some sort of summary has been given of the main results that have been arrived at in the branches of the subject with which they deal, and the purpose of this final chapter is not to give any general summary of results, but rather to look forward and to note the various directions in which investigation is progressing or is especially needed.

Cytology, in the sense in which the word is usually understood, has in the past been very largely a morphological and descriptive science; its aim has been to observe the structure of cells, to determine the parts of which they are composed, and to describe the changes through which they pass in the various phases of rest and of division. But as in other branches of biology, the study of morphology inevitably leads on to that of function. Anatomy is a necessary precursor of physiology, if the conclusions of physiology are to be more than superficial, but anatomy alone is an unsatisfying study if the functions of the organs remain unknown. And even more than the grosser anatomy of animals, cytology forces on its students the continual demand for explanation either in terms of function or by means of the still more fundamental laws of physics and chemistry. For, at least since the general acceptance of the principle of evolution, comparative anatomists have at times been content with purely morphological study, in the belief that they could thereby discover the phylogenetic relationships of different animal groups. But the science of comparative cytology is almost non-existent, with the result that 
without this aspect of the subject to distract them, cytologists are continually driven to ask for explanations in terms of functional or physical causes.

Very frequently the attempt to correlate cytological phenomena with the functions of the organism as a whole has been made in the opposite direction to that indicated above; instead of the cytologist looking for elucidation of his discoveries in terms of function, the investigator of function has turned to cytology for explanation of the facts that he observes. But in most of the chief advances that have been made, the first steps have been taken on the purely cytological side. This is seen especially in the subjects that have occupied cytologists more than any others in the last few years-the material basis of hereditary transmission and of the determination of sex. The foundations of modern ideas on the localisation of the factors for inherited characters was laid by Weismann, and though much of the theoretical superstructure built by him on insufficient factual evidence has proved unsound, his basal idea of the relation between these factors and the chromosomes is continually receiving fuller recognition. And Weismann was led to his idea of the germ-plasm directly from his work on the early origin of the germ-cells, and to his hypothesis of the function of the chromosomes from researches of cytologists on their behaviour in the maturation divisions. Similarly, the idea that the separation of the chromosomes in maturation is responsible for the segregation of Mendelian characters in the germ-cells of a heterozygote arose from the investigations of WrLson, Sutton and others on the spermatogenesis of Orthopterous and Hemipterous Insects, and the latest development of this theory-the chiasma type hypothesis of MORGAN - is based on the purely cytological investigations of JANSSENS. The same thing is true of the

D. C. 
hypothesis that sex is determined by specific chromosomes; the first suggestion on the subject came from MCCLUNG who was led to it from the study of the "accessory chromosome" in various insects. In general it is only after cytologists have discovered a possible basis for the explanation of some general function that a definite search can be made, with any prospect of success, for cytological facts that shall explain some specific phenomenon, for unless the investigator has some idea of what to expect, he is hardly likely to find his object. When, however, other facts are known which make some definite condition seem possible or probable in a specific case, cytological investigation may lead to the explanation sought for. Recent examples of this sort are the discovery by BRIDGES of non-disjunction of the sexchromosomes as an explanation of the exceptions to sexlimited inheritance in Drosopbila, and the finding of an unpaired or unequally paired chromosome in the females of certain Lepidoptera. In each of these cases the facts of heredity seemed to demand a definite cytological condition, and when examination was made the expected condition was found.

The study of heredity and sex-determination has had so large a share of recent biological research that it is not surprising that the attention of cytologists should have been especially attracted to this aspect of the subject, and this tendency has been accentuated by the comparative ease with which chromosomes are observed and the beauty of the mitotic process. There are, however, other directions in which research is no less needed. One of the most important is that of cell-differentiation. As was pointed out in the last chapter, the problem of differentiation is one of the fundamental problems of life; on the answer to the question of how differentiation is effected depends, to a great 
extent, the choice between a vitalistic and a mechanistic theory of life as a whole. The solution of this great problem is probably very distant, but clearly one of the conditions of approaching it is to discover the actual steps by which differentiation is accomplished. The answer to the question "In what manner?" does not necessarily give that of the question, "By what cause?"; it may be possible to describe every step in the process and yet to be ignorant of whether the causes at work are purely mechanical or not. But at the present time very little indeed is known of the simple stages of differentiation. Sometimes, as in the cleavage of Styela, some steps in the process can be seen, but even in such cases as this it is possible that the apparent clearness with which the changes can be watched is really misleading, and merely hides the more fundamental factors that lie behind. And in many cases in which a cell divides unequally it is possible to say no more than that a differential division has taken place; the nature of the unlikeness between the two cells is entirely beyond our present powers of analysis.

The study of differentiation leads the cytologist to the borderland between his territory and that of the experimental embryologist, and another subject that has attracted much attention in recent years may perhaps be regarded as carrying him across the frontier. This is the problem of the stimuli that lead to cell-division, and to the initiation of development in an egg. For a full elucidation of such questions the methods of the experimental embryologist are doubtless needed, but the subject is essentially one of cytology, and the chief hope for advance lies in the co-operation or combination of the two lines of investigation. In this respect science is like the boring of a tunnel; the workmen start far apart, and those at one end have no immediate knowledge of what those at the other end are doing, but 
success is only achieved, and a great human advance made, when they meet and discover that their work has all along been one. And when cytology becomes experimental, it will come into contact immediately, not only with other branches of Biology, but with the still more general facts and laws of Chemistry and Physics. Cytology touches at every point the fundamental problems of the chemical and physical basis of life, and whether the mechanistic or the vitalistic theory be the true one, it is equally important to discover the chemical and physical relations of living matter. Whether the living differs from the inorganic only in its greater complexity, or whether life is something unique and inexplicable in terms of matter and energy, it remains true that living things are composed of matter and obey the laws of energy, and in every branch of cytology the student is brought up against these laws for an explanation of the phenomena he observes. A conspicuous example of this is given by the various hypotheses of cell and nuclear division; osmosis, surface-tension, electricity, and a hypothetical form of energy named provisionally "mitokinetism" have all been invoked to account for the facts, and only a fuller knowledge of the action of physico-chemical forces in living matter can provide the answer to the riddle. Again, in the phenomena of fertilisation and parthenogenesis, some observers explain the facts in terms of chemistry, supposing that the spermatozoon or the parthenogenetic agent introduces substances into the egg, while others point to a physical explanation, and ascribe the changes to an alteration of, the electrical conductivity of the egg-surface. Even in the most elementary practice of cytology, in the fixation of cells for microscopic examination, it is only by some knowledge of the physics and chemistry of colloids that we can decide with confidence whether we are observing natural 
objects or artefacts; in general the technique is largely empirical, and could possibly be improved if this side of the subject were better known. Such examples 'could be multiplied indefinitely, and all indicate that the purely descriptive cytology of the past must give place to a more experimental science in the future, and that from the union of the two methods of work will be born the fuller knowledge for which we hope.

Lastly, further investigation is desirable in a direction quite different from the lines suggested above, and in a branch of cytology that has been scarcely mentioned in the preceding pages. At the present time the science of Comparative Cytology can hardly be said to exist, and yet, when the great results are borne in mind that have been obtained from the study of comparative anatomy of animals and plants, it can hardly be doubted that there is an important future for the comparative study of the cellular phenomena in the different groups. Perhaps the chief reason for the meagreness of our present knowledge is the remarkable uniformity of cytological behaviour not only in all groups of animals but also in many plants. If the Protozoa are excluded, the general structure of cells at rest and in division is extraordinarily similar in all groups of animals from the lowest to the highest, and differs only in minor points in the majority of plants. Even in the Protozoa essentially similar phenomena are found, though in their types of mitotic division, in the reconstitution of nuclei from chromidia, and in the great complication of the cell-body -if the Protozoan body is in fact a cell-they may show rather conspicuous deviation from the more usual type. There is, of course, immense variety of cytological type in the animal kingdom, but comparatively little has been done in the way of correlating this variety with the classification based on 
anatomical characters. A few attempts in this direction have been made. MEEk, for example, on the basis of measurements of the thickness of chromosomes in the metaphase of division, concludes that the chromosomes of the Protozoa are typically $0.2 \mathrm{I} \mu$ in width; that in those of the Coelenterata, Nemertinea and Echinodermata this width is doubled $(0.42 \mu)$ and that in all the chief higher Phyla (Nemathelminthes, Annelida, Arthropoda, Mollusca and Vertebrata) the width of the chromosomes is twice as great again, giving an average measurement of $0.83 \mu$. MEEK's conclusions have been criticised on the grounds that measurements of sufficient accuracy are hardly possible with existing methods, and that the sizes of the chromosomes vary according to the type of cell examined, but whether his results are substantiated or not, they suggest a wide and important field for further research. Comparative anatomy provides one of the chief foundations for the theory of evolution, and it can hardly be doubted that by a comparative study such as MEEK has made of a few forms and in respect of only certain characters, new and valuable information could be obtained with regard to the evolution of cell structure.

The successful prosecution of such investigations might lead very far towards a solution of one of the most fundamental and mysterious problems of biology, that of the nature of the earliest forms of living organisms. It has often been assumed that the first forms of life were composed of simple protoplasm, comparable with the most undifferentiated cytoplasm of simple embryonic cells. But this is a pure assumption; no such organism or undifferentiated cell is known, and it may be that in supposing life to have first arisen in a form of this kind, speculative biologists have been looking in the wrong direction. Present cytological knowledge gives little support for such a hypothesis, and 
it may be that a combination of comparative and experimental cytology will lead to a truer conception. In this connection reference must be made to Minchin's Presidential Address to Section D of the British Association in 1915, written very shortly before his death. It is impossible in a short space to give an adequate account of this most suggestive and illuminating paper, but its central idea may be shortly summarised. Minchin points to the facts that the nucleus, or, in some Protozoa, the chromatin granules (chromidia) which represent it, is essential to the life of the cell; that in the nucleus the chromosomes or other chromatin particles seem to be the essential portion; and that the chromatin particles are the only constituents of the cell that are not only universally present, but also seem to have a continuous existence and individuality through all cellgenerations. These facts suggest that the most primitive living organisms were composed, not of a homogeneous substance resembling cytoplasm, but of chromatin or something nearly allied to it. They were possibly very minute, even ultramicroscopic, and their nearest living relatives may be the ultramicroscopic and unfilterable organisms that are responsible for such diseases as Measles and Yellow Fever. From these primitive chromatinic corpuscles ("biococci") Minchin imagines that evolution may have proceeded on two lines. On the one hand they may have developed an enveloping membrane and have given origin to the Cocci and Bacilli known collectively as Bacteria. On the other hand they may have evolved an outer viscous or semi-fluid layer of "periplasm," and from being holophytic in habit have taken to ingesting solid food by means of this amoeboid covering, within which the biococci could multiply and form a group of chromatin granules enclosed in a mass of primitive cytoplasm. From such a condition it is not difficult 
to imagine the evolution of the more primitive forms of Protozoan cell, and by the aggregation or multiplication of the biococci to suppose that a simple nucleus could arise.

Evidently, as Minchin says, the cell and nucleus of the Metazoa are highly organised structures which must have passed through many stages of evolution before they reached their present form. To obtain clear indications of the more primitive condition the comparative cytologist will need to turn to the Protozoa, among which there is probably much greater variety of cell and nuclear structure than in all the Metazoa taken together. Even in the Protozoa, however, many kinds of differentiation have taken place in various directions, and the search for conditions that may be regarded as primitive is no easy one. On all grounds, however, it seems certain that just as the cytologist of the future whose interest is centred on function will be carried by his studies to the borderland between biology and physico-chemistry, so the student of cellular morphology will be driven to seek for the origins of cell-structure in the Protozoa, Protophyta and Bacteria.

Cytology in the last few years has been in a condition not unlike that of Zoology in the third quarter of the nineteenth century. After the publication of the Origin of Species morphological and systematic Zoology, carried away by an illuminating and unifying idea, devoted itself to the testing of the evolutionary theory and to seeking fresh evidence in its support, and allowed itself to become divorced from its natural partner, Physiology. So, since the discovery of a possible relation between Mendelian segregation and chromosome behaviour, and between specific chromosomes and sex-determination, descriptive cytology has been occupied very largely with the investigation of these problems, and has allowed the closely allied experi- 
mental science to pass into other hands. But zoology and physiology are essentially one, and each suffers from the division, inevitable though it was, that has come between them. If cytology is to avoid a similar misfortune, its students must keep in view the need for both the descriptive and comparative and the experimental methods, and remember that the bio-chemist and physicist are studying with flask and test-tube the same problems that they themselves are attacking with microscope and microtome. 


\section{BIBLIOGRAPHY}

\section{Text-books and Works of General Reference in English}

WiLson, F. B. The Cell in Development and Inheritance, 2nd Edn, pp. 483. New York, 1900, reprinted 1913. (The best general account and bibliography of Cytology up to the date of its publication.)

Morgan, T.H. Heredity and Sex, pp. 284. 2ndEdn. New York, I9I4. The Physical Basis of Heredity. Philadelphia, I9I9.

Sturtevant, A. H., Muller, H. J., and Bridges, C. B. The Mechanism of Mendelian Heredity, pp. 262. New York, I9I 5. (These books give a good summary of the chromosome theory of Hereditary Transmission and Sex-determination, and of the work on Drosopbila. They contain most useful bibliographies of the subject.)

Jenkinson, J. W. Experimental Embryology, pp. 341. Oxford, I909.

Driesch, H. The Science and Philosophy of the Organism. 2 vols. London, 1908.

Przibram, H. Experimental Zoology. Pt I. Embryogeny. Cambridge, 1908 .

Loeb, J. Artificial Parthenogenesis and Fertilization. Chicago, I9I3. Hegner, R. W. The Germ-cell cycle in Animals. New York, 1914. Doncaster, L. Chromosomes, Heredity and Sex. Quart. Journ. Micr. Sci: L1x. 1914, pp. 487-521. (A short summary of the chromosome theory of heredity and sex, with a list of the most important papers on the subject.)

- The Determination of Sex. Cambridge, 1915.

Goldschmidt, R. Theodor Boveri. Science, xi.rir. 19I6, pp. 263270. (An obituary notice of Boveri, including a useful summary of the chief steps in cytological research.)

\section{Books and Papers referred to in the text}

Agar, W. E. (I9II). The spermatogenesis of Lepidosiren paradoxa. Quart. Journ. Micr. Sci. LVII. p. I.

(I9I2). Transverse segmentation and internal differentiation of chromosomes. Quart. Journ. Micr. Sci. Lviri. p. 285.

Altmann, R. (1886). Studien über die Zelle. Leipzig.

--. (1887). Die Genese der Zellen. Leipzig.

Амма, K. (I9I I). Über die Differenzierung der Keimbahnzellen bei den Copepoden. Arch. Zellforsch. vi. p. 497. 
Artom, C. (1912). Analisi comparata della sostanza cromatica etc. dell' Uovo dell' Artemia. Arch. Zellforsch vir. p. 277.

von BaEHR, W. B. (I909). Die Oogenese bei einigen viviparen Aphiden, etc. Arch. Zellforsch. III.

- (1912). Contributions à l'étude de la caryocinèse, etc. La Cellule, xxvir. p. 385 .

Baltzer, F. (I9IC). Über die Beziehung zwischen dem Chromatin und der Entwicklung, etc. Arch. Zellforsch. v. p. 497.

Bataillon, E. (I910). Le problème de la fécondation...et la parthénogénèse traumatique. Arch. Zool. Exp. et Gén. vi. p. IOI.

Bateson, W. and Pellew, C. (1915). The Genetics of "Rogues" among culinary Peas. Journ. Genet. v. p. 14.

van Beneden,'E. and Neyt, A. (1887). Nouvelles Recherches sur la fécondation chez l'Ascaride mégalocéphale. Bull. Acad. Roy. Belg. vir.

Bonnevie, K. (1908 a). Chromosomenstudien. I. Arch. Zellforsch. I. p. 450.

P. (1908 b). Chromosomenstudien. II. Arch. Zellforsch. If. p. 201 .

BoverI, T. (I 888). Zellenstudien. Jena. Zeitschr. xxIr.

(1895). Über die Befruchtungs- und Entwicklungsfähigkeit kernloser Seeigeleier. Arch. Entwick. mech. II. p. 394.

(1903 a). Ergebnisse über die Konstitution...des Zellkerns. Fischer, Jena.

$-(1903 b)$. Über den Finfluss der Samenzelle auf die Larvencharaktere der Echiniden. Arch. Entwick. mech. xvı. p. 340.

- (1910). Über die Teilung centrifugierter Eier von Ascaris megalocephala. Arch. Entwick. mech. xxx. p. Ior.

(I9I I). Über das Verhalten der Geschlechtschromosomen bei Hermaphroditismus. Verhandl. Phys. Med. Ges. Würzburg, xıI. p. 83.

BRACHet, A. (1917). L'œuf et les facteurs de l'ontogénèse. Paris (Doin).

Brauer, A. (1893). Zur Kenntniss der Reifung des...Eies ron Artemia salina. Arch. Mikr. Anat. xLIII.

Bridges, C. B. (I9I6). Non-disjunction as proof of the chromosome theory of heredity. Genetics, I. Pp. I and 107.

Brues, C. T. (1903). A contribution to our knowledge of the Stylopidae. 7.ool. Jahrb. (Anat.), xviri. p. 255.

Buchner, P. (1910). Die Schicksale des Keimplasmas der Sagitta. Festschr. R. Hertwig. I. Jena, p. 236.

- (I9II). Die Reifung des Seesterneies bei experimenteller Parthenogenese. Arch. Zellforsch. vi. p. 577.

- (1918). Vergleichende Eistudien: Die akzessorischen Kerne des Hymenoptereneies. Arch. Mikr. Anat. xcr.

BüTschl, O. (1894). Investigations on Microscopic Foams and Protoplasm. Trans. E. A. Minchin. London. 
Carothers, E. E. (1917). The segregation and recombination of homologous chromosomes...in Acrididae. Journ. Morphol. xxvinr. p. 445 .

Child, C. M. (1907-8). Studies on the Relation between Amitosis and Mitosis. I-VI. Biol. Bull. xir., xirr. See also Biol. Bull. xvirI. rgIo, xxi. I9! I, and Anat. Anz. xxv. 1904, Xxx. 1907.

Conklin, E. G. (IgI2). Cell size and nuclear size. Journ. Exp. Zool. xiI. p. I.

(1917). Effects of centrifugal force on the structure and development of the eggs of Crepiaula. Journ. Exp. Zool. xxir. p. 3 II.

- (IgI8). Heredity and Environment in the Development of Men. Princeton (2nd Edn).

Davis, H. S. (1908). Spermatogenesis in Acrididae and Locustidae. Bull. Mus. Compar. Zool. Harvard, LiIr. p. 59.

Debaisieux, P. (1909). Les débuts de l'ovogénèse dans le Dytiscus marginalis. La Cellule, xxv.

Dein eCKa, D. (I9I2). Der Netzapparat von Golgi in einigen Epithelund Bindgewebezellen, etc. Anat. Anz. xu. p. 289.

Delage, Y. (1902-3). L'acide carbonique comme agent de choix de la parthénogénèse expérimentale, etc. C. R. Acad. Sci. 1902, 1903.

(1908). I es vrais facteurs de la parthénogénèse expérimentale. Arch. Zool. Exp. et Gén. Ser. Iv. T. vir.

Dobell, C. C. (I909). Chromidia and the Binuclearity Hypothesis. Quart. Journ. Micr. Sci. LiII. p. 279.

Doncaster, L. (1906). On the maturation of the Egg in the Tenthredinidae. Quart. Journ. Micr. Sci. xurx. p. 561.

- (I9IO). Gametogenesis and sex-determination in the Gall-fly Neuroterus. Pt I. Proc. Roy. Soc. B, 82, p. 88.

- (I9II). Gametogenesis and sex-determination in the Gall-fly Neuroterus. Pt II. Proc. Roy. Soc. B, 83, p. 476.

- (1916). Gametogenesis and sex-determination in the Gall-fly Neuroterus. Pt III. Proc. Roy. Soc. B, 89, p. 183.

(I914). On the relation between chromosomes, sex-limited transmission and sex-determination in Abraxas grossulariata. Journ. Genet. IV. p. I.

- (1915 a). The Determination of Sex. Cambridge.

$($ I9I $5 b)$. The relation between chromosomes and sex-determination in Abraxas. Nature, June 10, 1915.

- See also Harrison, J. W. H. and Doncaster, L.

Driesch, H. (1908). The Science and Philosophy of the Organism. Gifford Lectures, 1907-8. London.

Duesberg, J. (I910). Nouvelles Recherches sur l'appareil mitochondrial des cellules séminales. Arch. Zellforsch. vi. p. 40.

- (I9II). Plastosomen, "Apparato reticolare interno," und Chromidialapparat. Anat. Hefte: Ergebn. Anat. u. Entwick. xx. p. 566. 
Elpatiewsky, W. (1909). Die Urgeschlechtszellenbildung bei Sagitta. Anat. Anzeiger, xxxv. p. 226.

(1910). Entwicklungsgeschichte der Genitalprodukte bei Sagitta. Biol. Zeitschr. (Moscow), I. p. 333.

Farmer, J. B. and Moore, J. E. S. (1905). On the Meiotic Phase in Animals and Plants. Quart. Journ. Micr. Sci. xuvin.

Federley, H. (1913). Das Verhalten der Chromosomen bei der Spèrmatogenese von Pygaera. Zeitschr. f. indukt. Abstamm. Ix.p. I.

Fick, R. (1905). Betrachtungen über die Chromosomen, etc. Arch. Anat. und Physiol. (Anat.), 1905, Suppl. p. 179 (202).

Flemming, W. (i 882). Zellsubstanz, Kern und Zelltheilung.

- (1893). Zelle. Anat. Hefte, Abt. II. vol. III. p. 42.

Francotte, P. (1897). Recherches sur la maturation, etc. chez les Polyclades. Arch. Zool. Exp. et Gén. vi. Also Mém. Cour. Acad. Belg. Lv. 1897, p. 47.

Fries, W. (1908). Die Entwicklung der Chromosomen im Ei von Branchipus und der parthenogenetischen Generationen von Artemia. Arch. Zellforsch. I. p. 538.

Gallardo, A. (I909). La division de la Cellule, phénomène bipolaire, etc. Arch. Entwick. mech. xxviII. p. 125 .

Gaten By, J. B. (1917 a). The Cytoplasmic inclusions of the Germcells. Pt I. Quart. Journ. Micr. Sci. Lxil. p. 407.

- $(1917 b)$. The Cytoplasmic inclusions of the Germ-cells. Pt II. Quart. Journ. Micr. Sci. LXII. p. 555.

(IgI8 a). The Cytoplasmic inclusions of the Germ-cells. Pt III. Quart. Journ. Micr. Sci. LxIII. p. 197.

- (1918b). The Segregation of the Germ-cells in Trichogramma evanescens. Quart. Journ. Micr. Sci. Lxi11. p. 161.

- $(1918 c)$. Polyembryony in Parasitic Hymenoptera. Quart. Journ. Micr. Sci. Lxirr. p. 175.

(I919a). The Cytoplasmic inclusions of the Germ-cells. Pt IV. Quart. Journ. Micr. Sci. LxilI. p. 401.

- (1919b). The Cytoplasmic inclusions of the Germ-cells. Pt V. Quart. Journ. Micr. Sci. Lxin. p. 445.

(I919c). The Identification of Intracellular Structures. Journ. Roy. Micr. Soc. June, 1919, p. 93.

Gates, R. R. and Thomas, N. (1914). A cytological study of Oenothera mut. lata, etc. Quart. Journ. Micr. Sci. L1x. p. 523.

Godlewski, E. (1906). Untersuchungen über die Bastardierung der Echinoiden und Crinoiden Familien. Arch. Entwick. mech. xx. p. 579.

Goldschmidt, R. (1915). Some experiments on Spermatogenesis in vitro. Proc. Nat. Acad. Sci. (Washington), 1. p. 220.

(1916). Experimental Inter-sexuality and the Sex Problem. Amer. Nat. L. p. 705.

Gray, J. (1913). The effects of hypertonic solutions upon the fertilised eggs of Echinus. Quart. Journ. Micr. Sci. Lviri. p. 447. 
Gray, J. (1916). The Electrical Conductivity of Echinoderm eggs, etc. Phil. Trans. Roy. Soc. B, 207, No. 345, p. 48 r.

Grégoire, V. (1909). Les phénomènes de l'étape synaptique, etc. La Cellule, xxv. p. 87.

Gregory, R. P. (I9I4). On the genetics of tetraploid plants in Primula sinensis. Proc. Roy. Soc. B, 87, p. 484.

Günthert, T. (I9ro). Die Eibildung der Dytisciden. Zool. Jahrb. (Anat.), xxx. p. 301.

GUYFr, M. F. (1907). The development of unfertilized frog eggs injected with blood. Science, xxv. p. 9ro.

(I9I6). Studies on the chromosomes of the Common Fowl. Biol. Bull. xxxr.

HARDY, W. B. (1899). On the structure of cell protoplasm. Journ. Physiol. xxiv. p. 158.

(1913). Note on differences of electrical potential within the living cell. Journ. Physiol. xLvirr. p. 108.

Harrison, J. W. H. and Doncaster, L. (I9I4). On hybrids between moths of the Geometrid subfamily Bistoninae, etc. Journ. Genet. iiI. p. 234 .

Hartog, M. (1914). The true mechanism of mitosis. Arch. Entwick. mech. XL. p. 33 .

HeGNER, R. W. (I9I4). Studies on Germ-cells. I, II. Journ. Morphol. Xxv. pp. 375-509.

Heidenhain, M. (I894). Neue Untersuchungen über die Centralkörper, etc. Arch. Mikr. Anat. xLı11. p. 423.

Herbst, C. (1907). Vererbungsstudien. V. Arch. Entwick. mech. Xxiv. p. 185.

- (I909). Vererbungsstudien. VI. Arch. Entwick, mech. xxvir. p. 266.

HERlant, M. (I9I3). Étude sur les bases cytologiques...de la parthénogénèse expérimentale, etc. Arch. de Biol. xxvirr.

Hertwig, R. (1903). Über Korrelation von Zell- und Kerngrösse, etc. Biol. Centralbl. xxir. pp. 49, 108.

Janssens, F. A. (I909). Ira Théorie de la Chiasmatypie. La Cellule, xxv. p. 389.

JENKInson, J. W. (1904). The maturation and fertilisation of the egg of the Axolotl. Quart. Journ. Micr. Sci. xLvi1. p. 407.

Jordan, H. E. (I9I3). Amitosis in the epididymis of the Mouse. Anat. Anzeiger, xurr. p. 598.

Kahle, W. (I908). Paedogenese der Cecidomyiden. Zoologica, Igo8, P. I.

Kohlbrugge, J. H. F. (I9Io). Der Einfluss der Spermatoiden auf die Blastula. Arch. Mikr, Anat. Lxxv. p. 519.

Korschelt, E. and Heider, K. (I902). Lehrbuch der Vergleichenden Entwicklungsgeschichte. Allgemeiner Theil. Jena.

Kostanecki, K. (1904). Cytologische Studien an künstlich sich entwickelnden Eiern von Mactra. Arch. Mikr. Anat. Lxıv. p. I. 
Kostanecki, K. and Wierzejski, A. (I896). Über das Verhalten der sogen. achromatischen Substanzen im befruchteten Ei. (Physa fontinalis.) Arch. Mikr. Anat. xuvir. p. 309.

KrüGER, E. (1913). Fortpflanzung und Keimzellenbildung von Rhabditis aberrans. Zeit. Wiss. Zool. cv. p. 87.

KüнN, A. (1908). Die Entwicklung der Keimzellen in den parthenogenetischen Generationen der Cladoceren. Arch. Zellforsch. I. p. 538.

P. (1913). Die Sonderung der Keimesbezirke..,von Polyphemus pediculus. Zool. Jahrb. (Anat.), xxxv. p. 243.

Kupelwieser, H. (I909). Fintwicklungseregung bei Seeigeleiern durch Molluskensperma. Arch. Entwick. mech. xxvrr. p. 434.

- (I9I2). Weitere Untersuchungen über Entwicklungseregung durch stammfremde Spermatozoen. Arch. Zellforsch. vill. p. 352.

Kuschakewitsch, S. (1913). Studien über den Dimorphismus der männlichen Geschlechtselemente bei den Prosobranchia. Arch. Zellforsch. x. p. 237.

LeE, A. Bolles (I9II). La reduction numérique et la conjugaison des Chromosomes chez l'Escargot. La Cellule, xxvir. pp. 57, 221.

- (I912). La structure des Chromosomes et du noyau au repos. La Cellule, xxviri. p. 265.

LiLlie, F. R. (1909). Karyolinetic figures of centrifuged eggs, etc. Journ. Exp. Zool. xvir.

(1919). Problems of Fertilization. Chicago.

Lillie, R. S. (1916). The Physiology of Cell-division. VI. Journ. Exp. Zool. xxi. p. 369.

Loes, J. (1913). Artificial Parthenogenesis and Fertilization. Chicago. - (1918). Further Experiments on the Sex of Parthenogenetic Frogs. Proc. Nat. Acad. Sci. (Washington), rv. p. 60.

- and Bancroft, F. W. (1913). The sex of a parthenogenetic tadpole and frog. Journ. Exp. Zonl. xiv. p. 275.

McClung, C. E. (I902). The Accessory Chromosome-sex-determinant? Biol. Bull. III.

- (1905). The chromosome complex of Orthopteran spermatocytes. Biol. Bull. IX.

Mark, E. I. and Copeland, M. (1906). Some stages in the spermatogenesis of the Honey Bee. Proc. Amer. Acad. xlir. p. IOI.

Marchal, P. (1906). Recherches sur la biologie...des Hymenoptères parasites. II. Les Platygasters. Arch. Zool. Exp. et Gén. Iv. I906, p. $4^{85}$.

(I912). Notice sur les travaux scientifiques de M. Paul Marchal. Paris, 1912.

Marshall, W. S. (1907). Amitosis in the Malpighian tubules of the Walking-Stick. Biol. Bull. xrv. p. 89.

Matscheck, H. (1910). Eireifung bei Copepoden. Arch.Zellforsch.v. p. 43. 
MeEK, C. U. F. (I912). A metrical analysis of chromosome complexes, etc. Phil. Trans. Roy. Soc. B, 203, No. 294.

Metz, C. W. (19I4). Chromosome studies on the Diptera. I. Journ. Exp. Zool. xviII. p. 45.

(I9I6). Chromosome studies on the Diptera. II. Journ. Exp. Zool. xxi. p. 213.

Meves, F. (I900). Über den von La Valette St George entdeckten Nebenkern (Mitochondrienkörper) der Samenzellen. Arch. Mikr. Anat. Lvi.

- (I903). Über oligopyrene und apyrene Spermien. Arch. Mikr. Anat. LXI.

- (1907). Die Spermatocytenbildung bei der Honigbiene. Arch. Mikr. Anat. Lxx.

- (1912, I4). Verfolgung des sogenannten Mittelstückes des Echinidenspermiums, etc. Arch. Mikr. Anat. Lxxx. (II.) p. 8r and LXxxv. (II.) P. I.

Minchin, E. A. (1915). The Evolution of the Cell. Brit. Ass. Ann. Rept.: Pres. Address, Sect. D, 191 5.

Montgomery, T. H. (1910). On the dimegalous sperm and chromo somal variation in Euschistus, etc. Arch. Zellforsch. v. p 120.

- (I9II a). Spermatogenesis of an Hemipteran, Euschistus. Journ. Morphol. xxiI.

(I9I I b). Differentiation of the human cells of Sertoli. Biol. Bull. xxi. p. 367 .

(I9I2). Complete discharge of Mitochondria from the spermatozoon of Peripatur. Biol. Bull. xxir. p. 309.

Moore, J. E. S. and WaLKer, C. E. (1906). The meiotic process in the Mammalia. Cancer Research Lab., Liverpool University.

Morgan, T. H. (1909). The effects produced by centrifuging eggs before and during development. Anat. Record, III. p. 157 .

(1910). Cytological studies on centrifuged eggs. Journ. Exp. Zool. Ix. p. 593 .

(1915). The Predetermination of Sex in Phylloxerans and Aphids. Exp. Zool. xix. p. 289.

Sturtevant, A. H., Múller, H. J. and Bridges, C. B. (1915). The Mechanism of Mendelian Heredity. New York.

Morris, M. (1917). A cytological study of parthenogenesis in Cumingia. Journ. Exp. Zool. xxir. p. I.

Munson, J. P. (I9I2). A comparative study of the structure and origin of the Yolk Nucleus. Arch. Zellforsch. vir. p. 663.

Painter, T. S. (I9I8). Contributions to the study of cell mechanics. II. Journ. Exp. Zool. xxiv. p. 445.

PAYNE, F. (1909). Some new types of chromosome distribution and their relation to sex. Biol. Bull. xvi. p. II 9.

- (1910). The chromosomes of Acbolla multispinosa. Biol. Bull. xviri. p. 174 . 
Petrun kewitsch, A. (1904). Künstliche Parthenogenese. Zool. Jahrb. Suppl. vir. (Festschr. Weismann), p. 77.

Pinney, E. (1918). A study... of the chromatin...in Teleost hybrids. Journ. Exp. Zool. xxvi. p. 225.

Plovgh, H. H. (I9I7). The effect of temperature on crossing-over in Drosophila. Journ. Exp. Zool. xxiv. p. 147.

Popoff, M. (1908-9). Experimentelle Zell-studien. Arch. Zellforsch. I., III., IV.

(1907). Ei-bildung bei Paludina vivipara, etc. Arch. Mikr. Anat. Lxx. p. 43.

Przibram, H. (I908). Experimental Zoology. Pt I. Embryogeny. Cambridge.

Robertson, W. R. B (I915). Chromosome Studies. III. Journ. Morphol. xxvi. p. I09.

Rosen berg, O. (1904). Ber. Deutsch. Bot. Gesellsch. xxiI.

Roux, W. (1903). Über die Ursachen der Bestimmung der Hauptrichtungen des Embryos im Froschei. Anat. Anzeiger, xxiı. p. 66.

SaUnders, E. R. (I9II). Further studies on the inheritance of doubleness... in Stocks. Journ. Genet. I. p. 303.

Schaudinn, F. (1895). Über Plastogamie bei Foraminiferen. Sitzb. Naturforsch. Ges. Berlin, 1895, No. 10, p. 179 (187).

Schaxel, J. (1910). Die Beziehungen des Chromatins zum Cytoplasma, etc. Zool. Anzeiger, xxxvi. p. 33.

SchleIP, W. (1908). Vergleichende Untersuchungen der Eireifung bei... Ostracoden. Arch. Zellforsch. II. p. 390.

- (1909). Die Reifung des Eies von Rhodites, etc. Zool. Anzeiger, xxxv. p. 203.

- (I9I I). Über die Chromosomenverhältnisse bei Angiostomum. Arch. Zellforsch. vir. p. 87.

Schreiner, A. and K. E. (1906). Die Reifung der männlichen Geschlechtzellen, etc. Arch. de Biol. xxir.

Stiler, J. (I9I3). Geschlechtschromosomen bei Lepidopteren. Zool. Anzeiger, XuI. p. 246.

-(1917). Geschlechts-chromosomen untersuchungen an Psychiden. Zeitschr. f. indukt. Abst. xvir. p. 81 .

Silvestri, F. (1906-8). Contribuzioni alla conoscenza biologica degli Imenotteri parasiti. I-IV. Ann. R. Scuola d'Agric. Portici, vi., and Boll. Lab. R. Scuola d'Agric. Portici, I. and III.

- (1916). Sulla maturazione dell' ovo...nel Platygaster dryomyiae. Rendic. R. Acad. Lincei, xxv. p. I21.

Stevens, N. M. (1903). On the ovogenesis and spermatogenesis of Sagitta bipunctata. Zool. Jahrb. (Anat.), xvilı. p. 234.

- (1904). Further studies on the oogenesis of Sagitta. Zool. Jahrb. (Anat.), xxi. p. 343.

- (I90.5). Studies in spermatogenesis with especial reference to the accessory chromosome. Carnegie Inst. Washington Publ. 36.

D. C. 
Stevens, N. M. (1909). An unpaired chromosome in the Aphids. Journ. Exp. Zool. vi.

- (IgIO a). A note on reduction in the maturation of Male Eggs in Aphids. Biol. Bull. xvirr.

- $(1910 b)$. Further studies on reproduction in Sagitta. Journ. Morphol. xxi. p. 279.

Stout, A. B. (I9I2). The individuality of the chromosomes...in Carex aquatilis. Arch. Zellforsch. Ix. p. II4.

Sutron, W. S. (1903). The chromosomes in Heredity. Biol. Bull. iv. p. 231.

Tanaka, Y. (19r6). Genetic Studies on the Silkworm. Journ. Coll. Agr. Sapporo, Japan, vil. p. I29.

Teichman , E. (1903). Über die Beziehung zwischen Astrosphären und Furchen. Arch. Entwick. mech. xvi.

Townsend, C. O. (1897). Der Einfluss des Zellkerns auf die Bildung der Zellhaut. Jahrb. Wiss. Bot. xxx.

Walker, C. E. (1907). The Essentials of Cytology. London.

Weismann, A. (1885). Die Continuität des Keimplasmas als Grundlage einer Theorie der Vererbung. Jena, I 885 .

- (1893). The Germplasm. (Trans. Parker and Rönnfeldt.) London.

Wrison, E. B. (1896). On cleavage and mosaic-work. Arch. Entwick. mech. III. p. 19.

- (1900). The Cell in Development and Inheritance, 2nd Edn. New York.

- (I90I). Experimental Studies in Cytology. I, II. Arch. Entwick. mech. XII., xIII.

- (1904). Experimental Studies on Germinal Localisation. Journ. Exp. Zool. I. Pp. I and 197.

- (1906). Studies on Chromosomes. III. Journ. Exp. Zool. III. p. I.

P $(1909 a, b)$. Studies on Chromosomes. IV, V. Journ. Exp. Zool. vi.

(I9II). Studies on Chromosomes. VII. Journ. Morphol. xxir. p. 7I.

- (I9I2). Studies on Chromosomes. VIII. Journ. Exp. Zool. xIII. p. 345 .

Wilson, E. B. and Leaming, E. (1895). Atlas of Fertilisation. New York.

Wilson, E. B. and Mathews, A. P. (1895). Maturation, Fertilisation and Polarity in the Echinoderm Egg. Journ. Morphol. x.

Winge, O. (19I 7). The Chromosomes: their numbers and general importance. C. R. des Travaux Lab. Carlsberg, xir. p. I3I.

- (1919). On the relation between number of chromosomes and number of types. Journ. of Genet. viri. 1919, p. 132.

von Winiwarter, H. (I900). Recherches sur l'ovogénèse et l'organogénèse de l'ovaire des mammifères. Arch. de Biol. xviı. 


\section{BIBLIOGRAPHY}

von WiniWARTER, H. (I9I2). Études sur la spermatogénèse humaine. Arch. de Biol. xxvir. p. 91 .

(I9I4). L'hétérochromosome chez. le Chat. Bull. Acad. Roy. Belg. (Sci.), I9I4, p. 221.

Woltereck, R. (1898). Zur Bildung und Entwicklung des Ostracodeneies. Zeit. Wiss. Zool. LIv.

Zarnik, B. (I9II). Über den Chromosomencyclus bei Pteropoden. Verhandl. deutsch. Zool. Gesell. I9II, summarised by SchleIP, Ergebn. und Fortschr. d. Zool. III. I9I2, p. 250. 


\section{INDEX}

Abraxas, I 70, 231

Accessory chromosome, I 55

Acholla, 160

Acroblast, roo

Acrosome, 92, 99, I00, I02

Actinosphaerium, I6

AGAR, W. E., 63, 64, 69, 70

Allium, 196

Alternation of Generations, I 26

Altmann, R., 8

Amitotic division, 26, 27

Amma, K., I 82

Amphiaster, 53

Amphiuma, 196

Anaphase, 33

Anasa, ${ }^{5}{ }^{8}$

Annelids, ${ }^{4} 8$

Antedon, 242

Aphids, 81, I26, I63

Apyrene spermatozoa, 103

Arbacia, 245, 249, 250

Archoplasm, 48, 50, 98, 99, Iо I, IO2

Arion, 97

Arrhenotokous, I34

Artemia, I28, I29, I38, I93

Artificial Parthenogenesis, I40I 5 I

Artom, C., I 29

A scaris, 99, I6I, I 73-I75, I87, I9I, I95, I96, 25 I

Ascidians, 247-249, 25 I

Asters, 3I, 45, 48, 49, II 3

Autosomes, I59

Axial filament, 92, 94, 102

Axolotl, 46, 48

Bacillus, I $3 \mathbf{I}$

BAEHR, W. B. VON, I63

BALTZER, F., 2 IO, 2 II

BALY, E. C. C., $5^{8}$

Bat, I I note

BATAILlON, E., I49

BATESON, W., 2 I9, 222

Bee, 8I, I26, I 32, I 34, I 52, I 53

BENEDEN, E. VAN, 50
Bilharzia, 159

Bipolar field in mitosis, 52-54, 58

Birds, I69, 23I, 237

Biston, 86, 87, 200

Bivalent chromosomes, 7 I

Blatta, ${ }_{5} 8$

Bonnevie, K., I96, 218 note

Bouquet stage, $64,69,75$

Boveri, T., I6, 48, 50, 99, I67, I74, I88, I90, I9I, I95, 202, $207,208,209,249,25 I$

Brachet, A., II 3 note, I I9, I 20, 204

Branchipus, I 28

BRAUER, A., I 29

BRIDGES, C. B., 232-234, 240, 258

BRUES, C. T., 129

Buchner, P., Io8 note, I29, I 47 note, 148,176

BƯtschli, O., $8,5^{\circ}$

Calligrapha, I 79

Carex, I95

Carothers, E. E., 85

Cat, 230, 231

Cecidomyidae, I25, I 27, 138, 177

Cell, $\mathrm{I}-5$; Cell theory, $3-5$

Cell division, $26-4 \mathrm{I}, 54-58$

Cell organs, $13^{-25}$

Centriole, 44

Centrolecithal eggs, I I 8, I 2 I

Centrosome, 2I, 30, 42-47, 92-94, I02, I I 4, I I 5, I 47

Centrosphere, 44

Chaetopterus, I 48

Chalcididae, 82, 133

Chiasmatypy, 90, 225-229

Child, C. M., 26, 27

Chondriosomes, 2 I

Chromatin, I7, I 8, 20

Chromidia, 25

Chromosomes, 28-30; in Heredity, 206-240 conjugation of, I I5, I I 6 
Chromosomes, number of, I90193

shape and arrangement, 34-37. I99

width, 262

Chrysanthemum, 193

Coleoptera, 108, 157, I 59, I79

Conklin, E. G., Io, I5 note, 53 , $80,248,25 I$

Copepoda, 72,73, I 16,180

Crepidula, 10, 15 note, 80

Creseis, I 68

Crossing-over, 224-229

Crustacea, I09, I 27, I 38, I 54, I 82

Ctenophora, 253

Cumingia, 129, 249, 25 I

Cyclops, 180

Cynipidae, I 26, I3 I, I 34, I 35, I 36, I 39, I 53

Cytolysis, I 42

Cytophore, 104

Cytoplasm, 4, 5, 39-4 I, 54-58, $24^{\mathrm{I}-255}$

Daphnids, I 26, r 28, I 82

DebaisieuX, P., I 83

Delage, Y., I 46,147

Dentalium, 249, 253

Deutoplasm, 5, 10

Diakinesis, 68,7 I

Diaptomus, 180

Dictyosomes, 24

Differential division, 245-249, 259

Dileptus, 16

Diploid chromosomes, 83

Diplonema, diplotene, 67

Diptera, 85 note, I 59, I 72, I77I 79, I99, 200, 230

Dobell, C. C., 25 note

DRIESCH, H., I I 8, 244, 252, 254

Drosophila, 2I 4, 222-235, 240

DUESBERG, J., 25 I

Dytiscus, 183

Echinoids, 99, 107, I09, I I 2, I I 4, $122,138,140,146,199,202$, 208-2 I I, 242, 244, 252

Ectosomes, 182

Egg envelopes, 105, 109

Egg follicle, ro7

Egg structure, I05; 244

Electrical theory of mitosis, 52, $5^{8}$
Elimination of chromosomes, 210

Elpatiewsky, W., i 76

Entrance path of spermatozoon, I 99

Equatorial plate, 3 I

Equipotential system, I I 8, 253

Euschistus, 98, 100, I 58, 192

Facultative parthenogenesis, 13II 34

False fertilisation, 137

FARMER, J. B. and Moore, J. E. S., 63

FEDERLEY, H., 21 5, 240

Fertilisation, I I I-I I 7, I 44-I 46

by polar body, I 28-1 30

Fick, R., I 90 note, 22 I

Fixation, 5-I I

Flemming, W., 8, 50

Foraminifera, 14

Forficula, I 59

Francotte, P., 80

FrIES, W., 128

Frog, cleavage, I19, 249

parthenogenesis, I 49, I 5 I

Gallardo, A., 52

Gall-flies, I 26, I31, I 34, I 35, I 36,

I39, I 53

Gametes, maturation of, 59-90

Gametic coupling, 222

GatenBy, J. B., 95, 96, 97, 98, IOO, IOI, I02, I03 note, ro8, I 87,25 I

GATES, R. R., 2 I 2

Gemini, 7 I

Germ-cell determinants, I 72-187

Germ-cells, maturation of, 59-90

Germ track, I73

GiARDinA, A., I83

GODLEWSKI, E., 242

GoLDSCHMIDT, R., I03, 237

Golgi apparatus, II, 23, 24, 77, IOI, 102, 252

GRAY, J., I 44

GREGORY, R. P., 216,239

GÜNTHERT, T., I83

GUYER, M. F., I 49, 236 note

Haploid chromosomes, 83

HARDY, W. B., 8, 9, 17, 56 note, 64 note

HARRISON, J. W. H., 87

HARTOG, M., 49, 5I, 52, 58, 124 
HegneR, R. W., I77, I79, I80

Heidenhain, M., 50

Hemiptera, 98, 108, I 55, I 57 , I 58, I60, I97, I99

HerbSt, C., 208, 209

Hereditary transmission, 206240

HeRlant, M., 4I, 58, I49

Hermaphroditism, I67

HeRTWIG, R., I4-I7

Heterochromosomes, heterotropic chrom., I 55

Heterotype division, 66, 78, 88-90

Hieracium, I 29

HIRSCHLER, J., $25 \mathrm{I}$

Homoptera, I 38 (see Aphids)

Homotype division, $67,88,90$

Hybrids, chromosomes in, 86, 87 , 200, 208-2II, 2I 5, 240,242, 255

Hydatina, 249

Hymenoptera, 82, 88, 108, I26, I $31-139, I_{52}, I_{54}, I_{62}, I_{80}$

Idio-chromosomes, I57 (see $\mathrm{X}$ and Y-chromosomes)

Idiozome, 95 note, 98,99 , Iоo, 102

Individuality of chromosomes, I 88-205

Insects-ovaries, 75 , 108 polar divisions, 8I , I30-I39

Interzonal fibres, $3 \mathrm{I}$

JANSSENS, F. A., 89, 225, 257

Jenkinson, J. W., 46, 47, 49

Jessup, A. E., $5^{8}$

JORDAN, H. E., 26

KAHLE, W., I77

Karyokinesis, 26, 28-38

Karyoplasmatic ratio (Kernplasma relation), $\mathrm{I}_{4}-\mathrm{r} 7$

Karyosome, I 8, 28

KoHLBRUGGe, J. H. F., I II note

KOSTANECKI, K., I 49

KRÜGER, E., 138

KÜHN, A., I28, I 82

KuschaKeWITSCH, S., I03 note

Lee, A. Bolles, 69, 196, 198

Lepidoptera, 94, 96, 1о0-103, I69, 230-232, 237

Lepidosiven, 63-7I, 73, 84
Leptinotarsa, I79

Leptonema, leptotene, 67

LEUCKART, R., I77

LILLIE, F. R., 47, 53

LILlIE, R. S., 4 I note

Lines of force in mitosis, $48,5^{I}$, $52,5^{8}$

Linin, 17

Linkage, 222

Litomastix, $\mathbf{1} 80$

Locusta, I 59

LOEB, J., I40-I 5I, 242

Lygaeus, I 58

Lymantria, 237

Mactra, I 49

McClung, C. E., 64, 68, 258

Mammals, oogenesis, 77

sex chromosomes, 159, 230, 231

Man, I 59, 230, 23I

Mantle-fibres, 3I, 5I

Marchantia, I3

Matscheck, H., 2 I 8 note

Maturation of germ-cells, 59-90 of egg, 78-83, I I4, I I5, I27I 39

Mechanism of mitosis, 48-55

MEEK, C. U. F., 200, 262

Meiosis, meiotic phase, 63, 83-90 terminology of, 67

Mendelian factors, 212-240, 24I, 254

Metaphase, 33

Metaplastic structures, 5

Metapodius, I60, I92, I99

MetschNikOFF, E., I77

MetZ, C. W., 85, 235

Meves, F., 46 note, 96, 99, Io2, IO3 note, I 34

Miastor, $177, \mathrm{I} 86$

Micromitochondria, 97

Middle piece of spermatozoon, $43,46,47,92,99$, IO2, I I 4 , I I 5

Minchin, E. A., 263

Mitochondria, I I, 2I-23, 95-99, I02, 252

Mitokinetism, 52, $5^{8}$

Mitosis, 26, 28-38, 42-58

Mitosome, 95 note, 97,102

Mitotic spindle, 30,42

Molluscs, 97, IOI, IO3, I 48, 253

Monaster, 55 


\section{INDEX}

Moniezia, 26

MONTGOMERY, T. H., 69, 96, 98, $99,100,104,186,192$

MorGAN, T. H., 53, 127, 163 , I $65,222-230,249,257$

MORRIS, M., I 29

Multipolar spindles, 57, 123

Munson, J. P., 106

Myriapoda, 159

Mytilus, 1 38, 242

- Nebenkern, 50, 95 note, 97, IOI, IO2

Nematoda, 138, 159, 173

Nereis, 47,246

Neuroptera, 159

Neuroterus, I 36, 153

Non-disjunction, 232-234, 240

Nuclear division, $26-38,42-58$ membrane, 19,38

Nucleoli, 18

Nucleus, I 3-2I function of, $\mathrm{r}_{3}-\mathrm{r} 5$ structure of, 17-21, 195-199

Nutritive cells of ovary, 75,76 , 107

Nyssia zonaria, 87, 200

Oenothera, 193, 2 II, 239

Oligopyrene spermatozoa, I03

Oocytes, 76-79, 107

Oogenesis, 74-83, I05-I I0

Oogonia, 6I

Oosoma, 180

Ophryotrocha, 109

Organ-forming substances, II8, $247-251$

Orientation of cleavage planes and of embryo, II 7-120

Origin of zygotene threads, 69-7I

Orthoptera, 108, 131, I35, I55, I $58,197,199$

Ostracoda, 127,128

Pachynema, pachytene, 67

Paedogenesis, 125, 177

Painter, T. S., 58 note

Pairing of chromosomes, 69, 8490

Paludina, 85 note

Paramoecium, 16

Parasyndesis, parasynapsis, 68 , 70

Paris, 196, 198
Parthenogenesis, $125-139$

artificial, I 40-1 5 I

Payne, F., I60

Pediculus, 8r

Pentatoma, 158

Peripatus, 98, 99

Petrunkewitsch, A., 147 note

Phasmidae, I3I, 135

Phragmatobia, 169, I 70

Phyllaphis, 127, 163

Phylloxera, 165

Pinney, F., 211,242

Plasmosome, 18

Platygaster, 83

Platyhelminthes, I09.

Pluripolar spindles, 57, 123

Poecilosoma, 135

Polar bodies, 78 divisions of egg, 78-83, II I $15 ;$ 1 27-I 39

Polarity of egg, 244

Pole-plasm, 177

Polyphemus, 182

Polyspermy, 57, III, II 3, I22I 24, 202-204

Popoff, M., 16, 77

Primula, 193, 216, 239

Proctotrypidae, 82, I 33

Prophase, 133

Prostheceraeus, 80

Protenor, 158

Protoplasm, 4-I I

Protozoa, 1, 2, 4, 13, 14, 16, 25, $26,43,60,125,261,263,264$

Przibram, H., I2I note

Pseudoreduction, 68

Pteropoda, 168

Pygaera, 215

Pyrrhocoris, 158

Quadripolar spindle, 57, 123, 202

Reception cone, I 2

Reduction division, 59, 77, 126

Rhabditis, Rhabdonema, 138, I 39 , 167

Rhodites, I 35

ROSENBERG, O., 129

Rotifers, 126, 127, 1 28, 1 38, I 39 , I 54,249

Roux, W., I19, I 20

Sagitta, 173, 175-177

SAUNDERS, E. R., 219 
Saw-flies, 8I, I 3I, I 34, I 35

SchaudinN, F., I 4

SCHAXEL, J., IO7

SCHLEIP, W., I 28,167

SchreINER, A. and K. E., 69

Segmentation, 39-4I, 56, I I 7I24, 248

SEILER, J., I69

Sertoli cells, I03, I 86

Sex, I 5I, I 52-I 7 I, 229-238

Silvestri, F., 83, I33, I80

Simultaneity of division, I 22

Spermateleosis, 95

Spermatid, 62, 93-103

Spermatocyte, 62, 63, 94, 96

Spermatogenesis, 60-73, 90-103 of Hymenoptera, I34

Spermatogonia, 60, 63

Spermatozoon, structure of, 9I, 92

entrance into egg, I I I-I I 4

Spermiogenesis, 95

Sphaerechinus, 208-2II

Spiders, I 59

Spindle, mitotic, 30, 42

Spindle-bridge, Spindelrestkör-

Spireme, 68 per, 50, 95 note

Spirophase, 198

Stains, I I, I 2, 20

Starfish, I 29, I 30, I 47

Stevens, N. M., I 59, I63, I 76

Stick Insects, I3 I, I 35

Stout, A. B., r95

Strepsinema, strepsitene, 67

Strongylocentrotus, 208

Structure of protoplasm, 7-I I

Sturtevant, A. H., 224 note

Styela, 247-249, 25I

Stylopidae, I 29

Supernumerary chromosomes, I60

Surface tension, 4I, 56, I I 2, I 2 I

Sutton, W. S., 214,257

Synapsis, 56 note, 64,68

Syndesis, 68

Synizesis, 56 note, $64,68,75,127$. I 28

TAHARA, M., I 53

TANAKA, Y., 228
TeichmanN, E., 56

Telolecithal eggs, I 8

Telophase, 33

Telosyndesis, telosynapsis, 68

Tenthredinidae, 8I, I 3 I, I 34, I 35

Tetrads, 72

Tetraploid chromosomes, I93, 2 I 2

Tetraster, 57, I 23, 202

Thelykaryotic larvae, 209

Thelytokous parthenogenesis, I 35

Thompson, D’A. W., 54 note

Thrips, I3I

Thyanta, I6o

Thysanoptera, I 31

Tischler, G., I93

TownsEnd, C. O., I3

Trematoda, I 59

Triaster, Tripolar spindle, 57, 123

Trichogramma, 187

Tunicata, segmentation, 247249,25 I

Verson's cell, ro4

Vitelline membrane, I05, Iog. I I2

WALKer, C. E., 241

Wasp, I 32, I 34, I 53

WEISMANN, A., I72, I73, 206, 245,257

Wilson, E. B., I0, 69, I57, I60, I92, I96, I99, 246, 249, 257

WiNGE, O., I93, 22 I note

WINIWARTER, H. VON (DE), 67. 23 I note

WOLTERECK, R., I 28

X-chromosome, I55-I68, 230234

Xenos, I29, I38

Y-chromosome, I 57, 230-234

Yolk, 76, I06, Io8

lobe, 249

nucleus, 106, 108

ZARNIK, B., I 68

Zygonema, zygotene, 67

'Zygotene threads, origin of, 697 I 

